\title{
Digital twin of analogue man
}

Citation for published version (APA):

Heusinkveld, M. H. G. (2019). Digital twin of analogue man: development of a computational modelling platform to assess heart-vessel interaction in humans. [Doctoral Thesis, Maastricht University]. Ridderprint BV. https://doi.org/10.26481/dis.20191004mh

Document status and date:

Published: 01/01/2019

DOI:

10.26481/dis.20191004mh

Document Version:

Publisher's PDF, also known as Version of record

\section{Please check the document version of this publication:}

- A submitted manuscript is the version of the article upon submission and before peer-review. There can be important differences between the submitted version and the official published version of record.

People interested in the research are advised to contact the author for the final version of the publication, or visit the DOI to the publisher's website.

- The final author version and the galley proof are versions of the publication after peer review.

- The final published version features the final layout of the paper including the volume, issue and page numbers.

Link to publication

\footnotetext{
General rights Owners
rights.

- You may freely distribute the URL identifying the publication in the public portal. please follow below link for the End User Agreement:

www.umlib.nl/taverne-license

Take down policy

If you believe that this document breaches copyright please contact us at:

repository@maastrichtuniversity.nl

providing details and we will investigate your claim.
}

Copyright and moral rights for the publications made accessible in the public portal are retained by the authors and/or other copyright owners and it is a condition of accessing publications that users recognise and abide by the legal requirements associated with these

- Users may download and print one copy of any publication from the public portal for the purpose of private study or research.

- You may not further distribute the material or use it for any profit-making activity or commercial gain

If the publication is distributed under the terms of Article $25 \mathrm{fa}$ of the Dutch Copyright Act, indicated by the "Taverne" license above, 


\section{Digital Twin of Analogue Man}

Development of a Computational Modelling Platform to Assess Heart-Vessel Interaction in Humans 
ISBN 978-94-6375-509-2

Copyright (c) 2019 by Maarten Heusinkveld

All rights reserved. No part of this publication may be reproduced, stored in a retrieval system, or transmitted in any form or by any means, electronic, mechanical, photocopying, recording, or otherwise, without the prior written permission of the author.

Cover design by Evelien Jagtman | www.evelienjagtman.com

Printed by Ridderprint, the Netherlands | www.ridderprint.nl 


\title{
Digital Twin of Analogue Man
}

\section{Development of a Computational Modelling Platform to Assess Heart-Vessel Interaction in Humans}

\begin{abstract}
PROEFSCHRIFT
ter verkrijging van de graad van doctor aan de Universiteit Maastricht, op gezag van de Rector Magnificus, prof. dr. Rianne M. Letschert, volgens het besluit van het College van Decanen, in het openbaar te verdedigen op vrijdag 4 oktober 2019 om 12:00 uur
\end{abstract}

door

\section{Maarten Hendrik Gerd Heusinkveld}

geboren op 28 maart 1991

te Sittard, Nederland 


\section{Promotor}

Prof. dr. T. Delhaas

\section{Copromotores}

Dr. ir. K.D. Reesink

Dr. ir. W. Huberts

\section{Beoordelingscommissie}

Prof. dr. H.-P. Brunner-La Rocca (voorzitter)

Prof. dr. ir. A.L.A.J. Dekker

Prof. dr. D.J.G.M. Duncker (Erasmus Universiteit Rotterdam)

Prof. dr. ir. M. Mischi (Technische Universiteit Eindhoven)

Dr. R.J.M.W. Rennenberg

Financial support for the publication of this thesis as provided by Maastricht University is gratefully acknowledged. 


\section{Contents}

Chapter 1: General introduction

Chapter 2: Do treatment induced changes in arterial stiffness affect left ventricular structure? A meta-analysis

Chapter 3: Large vessels as a tree of transmission lines incorporated in the CircAdapt whole-heart model: A computational tool to examine heart-vessel interaction

Chapter 4: Augmentation index is not a proxy for wave reflection magnitude: Mechanistic analysis using a computational model

Chapter 5: Complementing sparse vascular imaging data by physiological adaptation rules

Chapter 6: Non-invasive model-based estimation of left ventricular end-diastolic pressure and compliance

Chapter 7: Uncertainty quantification and sensitivity analysis of an arterial wall mechanics model for evaluation of vascular drug therapies

Chapter 8: General discussion

Summary

Valorisation addendum

Acknowledgements (Dankwoord)

About the author 

General introduction 


\subsection{General remarks}

HIS thesis is entitled 'Digital Twin of Analogue Man: Development of a Computational Modelling Platform to Assess Heart-Vessel Interaction in

Humans'. The concept of using 'twins' is not new. For many years, plastic replicas of skeletons (i.e. 'physical twins') have been used in the classroom as well as in the general practitioner's office to explain aspects of the human skeletal anatomy. Physical twins were also used during NASA's Project Apollo in which two identical spacecraft were built, one to remain on the basis, which allowed engineers to recreate the conditions of the spacecraft during missions [11].

Digital twin technology is a novel concept originating from manufacturing industry [17]. A digital twin refers to a virtual or computational model containing a comprehensive physical and functional description of a component, product or system [11].

In this thesis, we apply the digital twin concept to cardiovascular research on the haemodynamic interaction between the heart and blood vessels. To emphasise the role of computational modelling, we first provide a timeline that outlines the technological developments that drive clinical data integration for improved interpretation.

\subsection{History of cardiovascular haemodynamics and imaging studies}

Already in ancient times, palpation of blood vessels has been performed as a method to diagnose disease. Ancient physicians and priests used the concept of measuring the 'state' of the heart through pulsating 'canals' in the head, hand and fingers [12]. It was the Greek physician Galen (129 - c. 210 ), who extensively characterised the cardiovascular system as being comprised of networks of arteries and veins. At that time, there was still no notion of the circular blood flow. Much later, William Harvey (1578-1657) was first to mention the blood circulation in his book De Motu Cordis, published in 1628. In contrast to Galen's understanding, Harvey discovered that 1) the arterial pulse is the result of an actively contracting heart and is not caused by intrinsic contraction of the arterial wall itself, 2) there exists a pulmonary circulation in which the right ventricle pumps blood through the lungs to the left ventricle, instead of venous blood passing through invisible pores in the interventricular septum, and 3) blood is continuously cycled through the body (i.e. the cardiovascular system is a closed-loop system), rather than being produced in the liver, distributed to the body, and vanished subsequently [2].

The first direct blood pressure measurements were performed by Stephen Hales (1677-1761) in England [30]. Hales performed arterial pressure measurements by inserting a brass cannula into a horse's carotid artery, which was connected to a glass tube to read out the height of the column of blood (Fig. 1.1). Hales's work spawned further developments for recording the arterial pressure waveform 
into the 1800s, which resulted in Scipione Riva-Rocci's method using an upper arm cuff to non-invasively measure the actual blood pressure in humans (first described in 1896). The early version of this device was capable of measuring peak (i.e. systolic) blood pressure. A few years later, following the discovery of the Korotkoff sounds in Russia, Riva-Rocci's method was improved to also include diastolic blood pressure measurements, based on simultaneous auscultation of the artery distally to the blood pressure cuff [10].

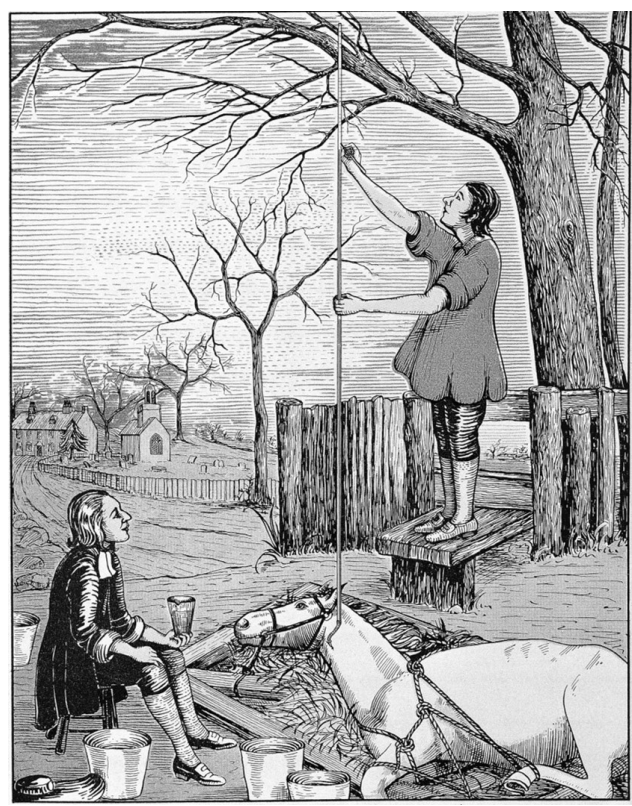

Figure 1.1: Reconstruction of Stephen Hales's method of in vivo blood pressure measurement, as performed in the carotid artery of a sedated horse. Reproduced with permission from Special Collections, University of Otago, Dunedin, New Zealand.

Other advances in cardiovascular research that took place around the 1900s include the invention of X-ray imaging. X-ray now allowed to obtain structural information of the heart and major blood vessels. An even greater breakthrough was the introduction of the electrocardiogram that provides functional information and is still used as the ground truth modality in emergency rooms and doctor's offices all over the world [3]. Cardiac ultrasound was invented in the 1950s and was at that time limited to describing the position of a cardiac structure over time (i.e. a so-called M-mode image). It took until the 1970 s for ultrasound scanners to appear to acquire two-dimensional images (i.e. B-mode) of the heart [44].

Vascular ultrasound emerged around the same time as cardiac ultrasound. Vascular research topics using ultrasound include assessment of the mechanical properties of arteries by studying arterial wall deformation under blood pressure pulsations $[16,19]$. Although most vascular ultrasound applications 
are non-invasive, intra-vascular methods using a transducer-mounted catheter are available to accurately evaluate the extent of atherosclerosis in arteries [46]. Among important clinical applications of vascular ultrasound is duplex ultrasound which provides two-dimensional greyscale images of blood vessels combined with a colour-coded map of the local blood flow velocity [13]. Currently, duplex ultrasound is a first-line imaging modality to assess amongst others carotid stenosis severity [28].

A more detailed assessment of significant stenoses can be obtained using computed tomography (CT) imaging [28]. CT is a minimally-invasive imaging technique based on acquiring multiple X-ray images at different angles and focal planes of a tissue [49]. Using a filtered back-projection algorithm, pioneered by the Austrian mathematician Johann Radon in 1917, the series of one-dimensional projections can be reconstructed into a two-dimensional image of the tissue [49]. In contrast to using duplex ultrasound for carotid stenosis assessment, CT additionally renders the three-dimensional structure of stenotic lesions, as reconstructed from multiple two-dimensional images. Furthermore, CT allows for characterisation of vessel wall composition (e.g. assessment of coronary calcium content).

Magnetic resonance imaging (MRI), based on the tissue-specific response of hydrogen nuclei to a magnetic field disturbance is a non-invasive imaging modality offering an anatomically detailed overview of the heart and blood vessels [49]. Furthermore, phase-contrast MRI is now widely used in cardiovascular MRI, providing a time-resolved quantitative assessment of blood flow in blood vessels as well as within the heart $[23,31]$.

Today, the variety of measurement devices (ultrasound machines, MRI scanners and blood pressure measurement devices) allow for a comprehensive and multi-faceted collection of clinical data. In theory, the multitude of clinical data enables researchers and clinicians to better understand and exploit the mechanisms of cardiovascular disease. However, interpretation of data is hindered by issues around mutual dependencies in the data, reliability, and accuracy, which makes correct diagnosis-making difficult [21]. Below, three illustrative examples of studies that are confronted with challenges regarding data interpretation are presented.

1. Cardiac diastolic function studies: In non-invasive settings, cardiac ultrasound is used for estimation of left ventricular (LV) filling pressure and diagnosis of diastolic dysfunction [34]. The E/e' ratio, based on Doppler ultrasound measurements of early mitral inflow velocity (E) and mitral annular early diastolic velocity ( $\mathrm{e}^{\prime}$ ), has been proposed as index for filling pressure [34]. However, meta-analysis of studies performing both cardiac ultrasound and cardiac catheterisation (i.e. considered gold standard) suggested that the $\mathrm{E} / \mathrm{e}^{\prime}$ ratio alone has limited value for assessment of LV filling pressure [42].

2. Arterial stiffness studies: Various arterial stiffness parameters (e.g. pulse pressure, regional pulse wave velocity or local distensibility) have been 
proposed as risk factors for cardiovascular diseases [45]. However, arterial stiffness measures as such do not discriminate underlying changes in arterial wall constituent properties (e.g. in collagen, elastin, or smooth muscle) [39]. Therefore, evaluation of changes in constituent properties is highly relevant for the development and monitoring of treatment [18].

3. Heart-vessel interaction studies: Presence of left ventricular hypertrophy $(\mathrm{LVH})$ is an important risk factor for cardiovascular disease and mortality [29]. Concentric left ventricular remodelling and LVH are common findings in hypertensive patients [15]. Therefore, consideration of LV afterload in relation to LV structure and function - as governed by heart-vessel interaction - is important for research regarding LVH. Despite the multitude of clinical data, it is controversial to what extent (ageing-induced) changes in afterload are resulting from earlier arrival of reflected waves or from reduced arterial compliance [7, 32, 37]. Hence, the afterload aspects striking 'the killing blow' to the LV remain unclear.

A solution to these challenges may be the development of elaborate statistical models that consider many variables and include interactions between variables. However, statistical modelling alone offers only a limited interpretation of causality, because these models are dependent on the population and circumstances they are based on $[1,21]$. In the field of biomedical engineering, computational models to capture physical and physiological relations, are being developed and applied $[21,36,41]$. In this thesis, we develop and assess physics-based computational models to interpret and estimate characteristics of heart-vessel interaction.

\subsection{Computational models of cardiovascular haemodynamics and their clinical applications}

\subsubsection{Models of the circulation}

Many physiological relationships related to cell, tissue, organ or the whole system can be approached by a mathematical relation. A computer model, composed of a network of applied physiological relationships, may simulate the cardiovascular system. As such, cardiovascular models are well-suited to test new hypotheses about mechanisms of cardiovascular diseases. Most cardiovascular models can roughly be assigned to three categories [43]. The first category consists of computationally expensive three-dimensional (3D) solid mechanics models, computational fluid dynamics models and fluid-structure interaction models, describing local stresses and strains, pressures and flow velocity fields, or all of the aforementioned physical quantities, respectively (Fig. 1.2) [9, 14, 25]. The second category is formed by computationally inexpensive zero-dimensional (0D) lumped parameter models, assuming uniform distributions of pressure and flow within entire compartments, e.g. the pulmonary circulation (Fig. 1.2). As such, lumped parameter models fail to take into account spatial variation of pressure and flow. The third is an intermediate category of the computationally expensive 3D models and 


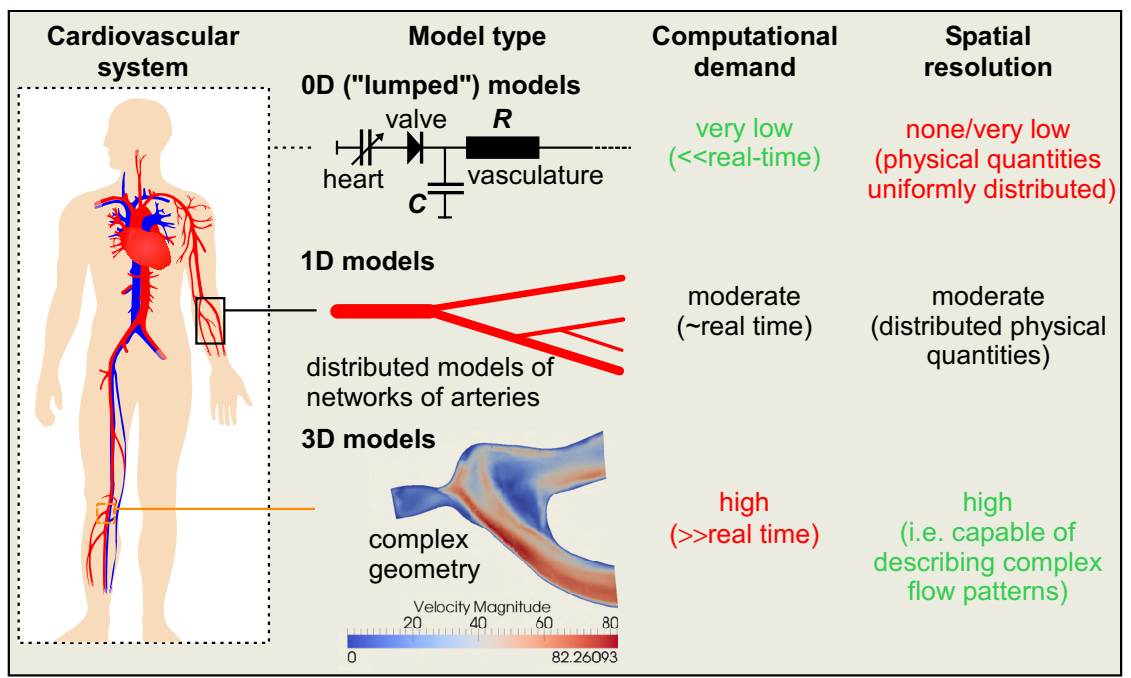

Figure 1.2: Dimensionality in cardiovascular modelling. Zero-dimensional (0D) or 'lumped' models consisting of a limited number of model parameters, (e.g. describing vascular resistance $(R)$ and compliance $(C)$ ), consider uniform distributions of physical quantities (e.g. pressure or flow), have a low computational demand (e.g. run faster than real-time), but lack spatial detail. One-dimensional (1D) or 'distributed' models allow calculation of physical quantities along a single spatial direction at the cost of a moderate increase in computational demand. Three-dimensional (3D) models offer the highest spatial resolution, capable of calculating complex patterns of physical quantities.

simplified 0D models, comprising of one-dimensional (1D) distributed models that simulate wave transmission in networks of blood vessels (Fig. 1.2) [26, 40].

As a general rule, the choice of model dimensionality is determined by the required spatial and temporal resolution, which in turn is determined by the research question or clinical application [43].

In this thesis, we consistently use 0D models of cardiac mechanics and distributed models of wave transmission. The CircAdapt platform, an in-house developed cardiovascular model, has been successfully applied so far for simulating haemodynamics and LV adaptation processes during valvular pathologies and cardiac conductance disorders $[27,38,48]$. Its cardiac model describes a simplified cardiac geometry and assumes the cardiac chambers to be surrounded by contractile cardiac walls consisting of myofibres [48]. The myofibres are described using the one-fibre model [5], which relates myofibre stress to cavity pressure using the assumption that myofibre stress is homogeneously distributed within the myocardial wall. Furthermore, instead of relying on input from imaging techniques such as ultrasound or MRI, cardiac geometry (i.e. wall thicknesses and cavity volumes) is determined using cardiac adaptation rules [6]. A current limita- 
tion of the CircAdapt model is that it does not yet contain a description of vascular wave transmission, which is believed to be important in studies on heart-vessel interaction (Section 1.2).

\subsubsection{Patient-specific model-based characterisation of cardiovascular structure and function}

In daily clinical practice, treatments are often based on the 'one-size-fits-all' paradigm in which results from clinical trials are used to define cut-off values for risk-stratification and clinical decision-making. Often, cut-off values are based on statistical associations between a clinical measure (e.g. systolic blood pressure by arm cuff) on the one hand, and a clinical end-point (e.g. heart failure or stroke) on the other. For some diseases like acute myocardial infarction, cut-off values exist that are useful for clinical decision-making [4, 47]. However, for complex diseases such as heart failure, cut-off values may lack precision because of the multi-factorial nature of the disease or the indirect description of a cut-off value as a marker for disease. Imputation or tuning computational models with or to a (subset of) clinical data could improve clinical decision-making. To this end, roughly two modelling approaches can be distinguished.

\section{Forward approach}

Personalised modelling can be performed using a forward approach [24, 33, 39], by substituting generic or population-averaged model parameters with their patient-specific counterparts, obtained or derived using measurements. Such a personalised model provides additional direct information on the state of the cardiovascular system by simulating variables (e.g. pressures, flows and material stresses) that cannot be routinely measured.

\section{Inverse approach}

An inverse approach $[8,24,39]$ may be employed in which model parameters are tuned so that model simulations and patient measurements agree. In case the model parameters represent e.g. passive or active properties of tissue, such fitting procedure provides model-based estimates of those properties based on patient measurements. In previous works, the inverse approach has been proposed to facilitate a constitutive interpretation of clinical arterial stiffness measurements [39].

\section{Limitations of patient-specific modelling}

Difficulties with respect to patient-specific modelling are 1) a lack of clinical data available for model parametrisation, 2) measurement uncertainty associated with the clinical data, and 3) a mismatch between available clinical data and required clinical data to unambiguously estimate the unknown model parameters. 


\subsection{Aim and outline of this thesis}

The aim of this thesis is to develop and evaluate a computational modelling platform to assess heart-vessel interaction. The developed models were employed in two use-cases concerning patient-specific modelling: 1) non-invasive computational methods to allow screening for LV diastolic abnormalities and 2) noninvasive identification of arterial wall mechanical properties to assess the effect of (novel) vascular drugs on cardiovascular system dynamics. The structure of this thesis is as follows.

In Chapter 2, we perform a meta-analysis of clinical intervention studies with arterial and LV structure/function as (primary) outcomes. This study serves as an important starting point to investigate heart-vessel interaction. In this review, we aim to evaluate, using clinical evidence, the current pathophysiological paradigm that links arterial stiffening and LV hypertrophy/failure [50].

In Chapter 3, we extend the existing CircAdapt computational model with a module describing arterial and venous wave transmission. The extended model is subjected to a relevant test-case, simulating the effect of systemic arterial hypertension on 1) global arterial and venous haemodynamics, and 2) changes in local wave dynamics at the level of the aorta. In Chapter 4, we use the newly extended circulatory model to critically evaluate the augmentation index (AIx). The AIx is a well-known and widely reported metric, deemed to capture the effect of increased cardiac workload due to wave reflections. However, its validity as a proxy for wave reflection magnitude has been questioned [22].

In Chapter 5, we model the adaptive capacity of blood vessels, making them respond to flow and pressure stimuli by changes in radius and wall thickness of blood vessels. We integrate the adaptation rules into a previously developed pulse wave propagation model [26], and assess whether the PWP-adaptation framework could be utilised to extrapolate incomplete patient-specific data sets. Modelbased estimates are compared to dedicated ultrasound and MRI measurements, as obtained in ten healthy volunteers.

In Chapter 6, we employ the extended CircAdapt model to non-invasively estimate $\mathrm{LV}$ end-diastolic pressure $\left(p_{\text {ed }}\right)$ and compliance $\left(C_{\text {ed }}\right)$ from routine measurements of blood pressure and echocardiographic recordings. This approach could improve the currently used method of assessing increased LV filling pressure, which reportedly lacks specificity, i.e. echocardiographic $\mathrm{E} / \mathrm{e}^{\prime} \neq p_{\text {ed }} \neq C_{\text {ed }}$ [42].

In Chapter 7, we model carotid arterial wall mechanics using a constitutive model that simulates stress-strain behaviour of the arterial collagen and elastin [20]. For constitutive interpretation of clinical arterial stiffness data, an inverse approach is utilised which enables estimation of constitutive properties. According to modelling standards [35], we perform uncertainty quantification to determine uncertainty in model-estimated constitutive property estimation.

This thesis concludes with a general discussion in Chapter 8, where we put the developments in the context of a multi-scale modelling platform and its utility for further mechanistic studies into heart-vessel interaction. 


\section{References}

[1] H. Aguinis, C. A. Pierce, F. A. Bosco, D. R. Dalton, and C. M. Dalton. Debunking myths and urban legends about meta-analysis. Organizational Research Methods, 14(2):306-331, 2011.

[2] W. C. Aird. Discovery of the cardiovascular system: from Galen to William Harvey. Journal of Thrombosis and Haemostasis, 9(s1):118-129, 2011.

[3] M. AlGhatrif and J. Lindsay. A brief review: history to understand fundamentals of electrocardiography. Journal of Community Hospital Internal Medicine Perspectives, 2(1):14383, 2012.

[4] E. A. Amsterdam, N. K. Wenger, R. G. Brindis, D. E. Casey, T. G. Ganiats, D. R. Holmes, A. S. Jaffe, H. Jneid, R. F. Kelly, M. C. Kontos, et al. 2014 AHA/ACC guideline for the management of patients with non-ST-elevation acute coronary syndromes: a report of the American College of Cardiology/American Heart Association Task Force on Practice Guidelines. Journal of the American College of Cardiology, 64(24):e139-e228, 2014.

[5] T. Arts, P. H. M. Bovendeerd, F. W. Prinzen, and R. S. Reneman. Relation between left ventricular cavity pressure and volume and systolic fiber stress and strain in the wall. Biophysical Journal, 59(1):93-102, 1991.

[6] T. Arts, J. Lumens, W. Kroon, and T. Delhaas. Control of whole heart geometry by intramyocardial mechano-feedback: A model study. PLOS Computational Biology, 8(2):e1002369, 2012.

[7] A. J. Baksi, T. A. Treibel, J. E. Davies, N. Hadjiloizou, R. A. Foale, K. H. Parker, D. P. Francis, J. Mayet, and A. D. Hughes. A meta-analysis of the mechanism of blood pressure change with aging. Journal of the American College of Cardiology, 54(22):2087-2092, 2009.

[8] H. T. Banks, M. Davidian, J. R. Samuels, and K. L. Sutton. An inverse problem statistical methodology summary. In Mathematical and Statistical Estimation Approaches in Epidemiology, pages 249-302. Springer, 2009.

[9] Y. Bazilevs, M. C. Hsu, D. J. Benson, S. Sankaran, and A. L. Marsden. Computational fluid-structure interaction: methods and application to a total cavopulmonary connection. Computational Mechanics, 45(1):77-89, 2009.

[10] J. Booth. A short history of blood pressure measurement. Proceedings of the Royal Society of Medicine, 70(11):793-799, 1977.

[11] S. Boschert and R. Rosen. Digital twin-the simulation aspect. In Mechatronic Futures, pages 59-74. Springer, 2016.

[12] B. Ebbell. The Ebers Papyrus. The Greatest Egyptian Medical Document. London: H. Milford and Oxford University Press, 17:123, 1937.

[13] W. E. Faught, M. A. Mattos, P. S. van Bemmelen, K. J. Hodgson, L. D. Barkmeier, D. E. Ramsey, D. S. Sumner, et al. Color-flow duplex scanning of carotid arteries: new velocity criteria based on receiver operator characteristic analysis for threshold stenoses used in the symptomatic and asymptomatic carotid trials. Journal of Vascular Surgery, 19(5):818-828, 1994.

[14] C. A. Figueroa, I. E. Vignon-Clementel, K. E. Jansen, T. J. Hughes, and C. A. Taylor. A coupled momentum method for modeling blood flow in three-dimensional deformable arteries. Computer Methods in Applied Mechanics and Engineering, 195(41-43):5685-5706, 2006. 
[15] A. Ganau, R. B. Devereux, M. J. Roman, G. De Simone, T. G. Pickering, P. S. Saba, P. Vargiu, I. Simongini, and J. H. Laragh. Patterns of left ventricular hypertrophy and geometric remodeling in essential hypertension. Journal of the American College of Cardiology, 19(7):1550-1558, 1992.

[16] A. R. Grahn, M. H. Paul, and H. U. Wessel. A catheter-tip ultrasonic transducer for measuring pulsatile aortic diameter and pressure elastic modulus. Biomedizinische Technik/Biomedical Engineering, 19(1):31-38, 1974.

[17] M. Grieves and J. Vickers. Digital twin: Mitigating unpredictable, undesirable emergent behavior in complex systems. In Transdisciplinary perspectives on complex systems, pages 85-113. Springer, 2017.

[18] M. H. G. Heusinkveld, S. Quicken, R. J. Holtackers, W. Huberts, K. D. Reesink, T. Delhaas, and B. Spronck. Uncertainty quantification and sensitivity analysis of an arterial wall mechanics model for evaluation of vascular drug therapies. Biomechanics and Modeling in Mechanobiology, 17(1):55-69, 2018.

[19] A. P. G. Hoeks, C. J. Ruissen, P. Hick, and R. S. Reneman. Transcutaneous detection of relative changes in artery diameter. Ultrasound in Medicine \& Biology, 11(1):51-59, 1985.

[20] G. A. Holzapfel, T. C. Gasser, and R. W. Ogden. A new constitutive framework for arterial wall mechanics and a comparative study of material models. Journal of Elasticity and the Physical Science of Solids, 61(1-3):1-48, 2000.

[21] W. Huberts, S. G. H. Heinen, N. Zonnebeld, D. A. F. van den Heuvel, J.-P. P. de Vries, J. H. M. Tordoir, D. R. Hose, T. Delhaas, and F. N. van de Vosse. What is needed to make cardiovascular models suitable for clinical decision support? A viewpoint paper. Journal of Computational Science, 24: 68-84, 2017.

[22] A. D. Hughes, C. Park, J. Davies, D. Francis, S. A. M. Thom, J. Mayet, and K. H. Parker. Limitations of augmentation index in the assessment of wave reflection in normotensive healthy individuals. PLOS ONE, 8(3):e59371, 2013.

[23] V. P. Kamphuis, A. A. Roest, N. Ajmone Marsan, P. J. van den Boogaard, L. J. M. Kroft, J.-P. Aben, J. J. Bax, A. de Roos, H. J. Lamb, and J. J. M. Westenberg. Automated cardiac valve tracking for flow quantification with four-dimensional flow MRI. Radiology, 290(1):70-78, 2018.

[24] R. C. P. Kerckhoffs. Patient-specific modeling of the cardiovascular system: technology-driven personalized medicine. Springer Science \& Business Media, 2010.

[25] R. C. P. Kerckhoffs, M. L. Neal, Q. Gu, J. B. Bassingthwaighte, J. H. Omens, and A. D. McCulloch. Coupling of a 3D finite element model of cardiac ventricular mechanics to lumped systems models of the systemic and pulmonic circulation. Annals of Biomedical Engineering, 35(1):1-18, 2007.

[26] W. Kroon, W. Huberts, E. M. H. Bosboom, and F. N. van de Vosse. A numerical method of reduced complexity for simulating vascular hemodynamics using coupled 0D lumped and 1D wave propagation models. Computational and Mathematical Methods in Medicine, 2012.

[27] N. H. L. Kuijpers, E. Hermeling, J. Lumens, H. M. M. ten Eikelder, T. Delhaas, and F. W. Prinzen. Mechano-electrical coupling as framework for understanding functional remodeling during LBBB and CRT. American Journal of Physiology-Heart and Circulatory Physiology, 306(12): H1644-H1659, 2014.

[28] S. Lepidi, H. S. Markus, D. J. McCabe, J. Roy, H. Sillesen, J. C. van den Berg, F. Vermassen, J. Archie, S. Bellmunt, A. Chaudhuri, et al. Editor's choice: Management of atherosclerotic carotid and vertebral artery disease: 2017 Clinical Practice Guidelines of the European Society for Vascular Surgery (ESVS). European Journal of Vascular and Endovascular Surgery, 55:3e81, 2018. 
[29] D. Levy, R. J. Garrison, D. D. Savage, W. B. Kannel, and W. P. Castelli. Prognostic implications of echocardiographically determined left ventricular mass in the Framingham Heart Study. New England Journal of Medicine, 322(22):1561-1566, 1990.

[30] 0. Lewis. Stephen Hales and the measurement of blood pressure. Journal of Human Hypertension, 8(12):865-871, 1994.

[31] J. Lotz, C. Meier, A. Leppert, and M. Galanski. Cardiovascular flow measurement with phasecontrast MR imaging: basic facts and implementation. Radiographics, 22(3):651-671, 2002.

[32] G. F. Mitchell, H. Parise, E. J. Benjamin, M. G. Larson, M. J. Keyes, J. A. Vita, R. S. Vasan, and D. Levy. Changes in arterial stiffness and wave reflection with advancing age in healthy men and women: the Framingham Heart Study. Hypertension, 43(6):1239-1245, 2004.

[33] R. Mukkamala and R. J. Cohen. A forward model-based validation of cardiovascular system identification. American Journal of Physiology-Heart and Circulatory Physiology, 281(6):H2714-H2730, 2001.

[34] S. F. Nagueh, O. A. Smiseth, C. P. Appleton, B. F. Byrd, H. Dokainish, T. Edvardsen, F. A. Flachskampf, T. C. Gillebert, A. L. Klein, P. Lancellotti, et al. Recommendations for the evaluation of left ventricular diastolic function by echocardiography: an update from the American Society of Echocardiography and the European Association of Cardiovascular Imaging. European Journal of Echocardiography, 17(12):1321-1360, 2016.

[35] National Research Council. Assessing the reliability of complex models: mathematical and statistical foundations of verification, validation, and uncertainty quantification. National Academies Press, 2012.

[36] S. A. Niederer, J. Lumens, and N. A. Trayanova. Computational models in cardiology. Nature Reviews Cardiology, 16(2):100-111, 2018.

[37] M. F. O’Rourke and W. W. Nichols. Aortic diameter, aortic stiffness, and wave reflection increase with age and isolated systolic hypertension. Hypertension, 45(4):652-658, 2005.

[38] G. Palau-Caballero, J. Walmsley, J. Gorcsan, J. Lumens, and T. Delhaas. Abnormal ventricular and aortic wall properties can cause inconsistencies in grading aortic regurgitation severity: A computer simulation study. Journal of the American Society of Echocardiography, 29(11): 1122-1130, 2016.

[39] K. D. Reesink and B. Spronck. Constitutive interpretation of arterial stiffness in clinical studies -a methodological review. American Journal of Physiology-Heart and Circulatory Physiology, 316(3): H693-H709, 2018.

[40] P. Reymond, F. Merenda, F. Perren, D. Rufenacht, and N. Stergiopulos. Validation of a onedimensional model of the systemic arterial tree. American Journal of Physiology-Heart and Circulatory Physiology, 297(1):H208-H222, 2009.

[41] A. X. Sarkar, D. J. Christini, and E. A. Sobie. Exploiting mathematical models to illuminate electrophysiological variability between individuals. The Journal of Physiology, 590(11):2555-2567, 2012.

[42] O. F. Sharifov, C. G. Schiros, I. Aban, T. S. Denney Jr, and H. Gupta. Diagnostic accuracy of tissue Doppler index E/e' for evaluating left ventricular filling pressure and diastolic dysfunction/heart failure with preserved ejection fraction: A systematic review and meta-analysis. Journal of the American Heart Association, 5(1):e002530, 2016.

[43] Y. Shi, P. Lawford, and R. Hose. Review of zero-D and 1-D models of blood flow in the cardiovascular system. Biomedical Engineering Online, 10(1):33, 2011. 
[44] S. Singh and A. Goyal. The origin of echocardiography: a tribute to Inge Edler. Texas Heart Institute Journal, 34(4):431, 2007.

[45] R. R. Townsend. Arterial stiffness: recommendations and standardization. Pulse, 4(Suppl. 1): 3-7, 2016.

[46] E. M. Tuzcu, O. Bayturan, and S. Kapadia. Coronary intravascular ultrasound: a closer view. Heart, 96(16):1318-1324, 2010.

[47] R. Twerenbold, J. Boeddinghaus, T. Nestelberger, K. Wildi, M. R. Gimenez, P. Badertscher, and C. Mueller. Clinical use of high-sensitivity cardiac troponin in patients with suspected myocardial infarction. Journal of the American College of Cardiology, 70(8):996-1012, 2017.

[48] J. Walmsley, T. Arts, N. Derval, P. Bordachar, H. Cochet, S. Ploux, F. W. Prinzen, T. Delhaas, and J. Lumens. Fast simulation of mechanical heterogeneity in the electrically asynchronous heart using the multipatch module. PLOS Computational Biology, 11(7):e1004284, 2015.

[49] J. G. Webster. Medical instrumentation application and design. John Wiley \& Sons, 2009.

[50] N. Westerhof and M. F. O'Rourke. Haemodynamic basis for the development of left ventricular failure in systolic hypertension and for its logical therapy. Journal of Hypertension, 13(9):943-952, 1995. 


\section{Do treatment induced changes in arterial stiffness affect left ventricular structure? A meta-analysis}

The contents of this chapter are based on:

Koen M. van der Waaij, ${ }^{1,2, *}$ Maarten H. G. Heusinkveld, ${ }^{1, *}$ Tammo Delhaas, ${ }^{1}$ Abraham A. Kroon, ${ }^{2}$ Koen D. Reesink ${ }^{1}$

Do treatment induced changes in arterial stiffness affect left ventricular structure? A meta-analysis. Journal of Hypertension (2019) 37(2):253-263

\footnotetext{
${ }^{1}$ Department of Biomedical Engineering, CARIM School for Cardiovascular Diseases, Maastricht University, Maastricht, The Netherlands.

${ }^{2}$ Department of Internal Medicine, CARIM School for Cardiovascular Diseases, Maastricht University, Maastricht, The Netherlands.

${ }^{*}$ These authors contributed equally to this work.
} 


\subsection{Abstract}

Vascular research demonstrated that pulse wave velocity (PWV), a measure of arterial stiffness, is inherently blood pressure-dependent. Considering the hypothesised pathophysiological chain of increased arterial stiffness leading to increased blood pressure load with consequent left ventricle hypertrophy (LVH) development, we conducted a systematic review of antihypertensive and lifestyle intervention studies to determine the association between on the one hand changes in arterial stiffness and blood pressure, and on the other hand changes in LV mass (LVM).

Using PubMed, EMBASE, Cochrane and Web of Science, we identified 23 studies, containing 2573 patients. Studies reported changes in arterial stiffness (assessed by means of PWV), systolic- and diastolic blood pressure (SBP, DBP), and LVM index (LVMI), respectively.

Statistically significant reductions in SBP, PWV and LVMI were reported in 16, 14, and 20 studies, respectively. Pooled analysis of studies showed that the proportion in SBP reduction did not correlate significantly to the proportion in reductions of the other two variables. On the other hand, we found a significant positive correlation $(r=0.61, p=0.003)$ between arterial stiffness and LVM, expressed as a relevant reduction in LVMI of $6.9 \mathrm{~g} / \mathrm{m}^{2}$ per $1.0 \mathrm{~m} / \mathrm{s}$ reduction in PWV.

Our findings provide evidence that a decrease in arterial stiffness is associated with reduction of LVM. To investigate whether there exists a causal relation between LVH due to arterial stiffness increases and in turn blood pressure load increases, future studies should strive for a multiple follow-up design and use of blood pressure-independent or corrected stiffness indices. 


\subsection{Introduction}

URRENTLY, cardiovascular disease (CVD) is the number one killer of humans worldwide, responsible for 17.9 million deaths (approximately $30 \%$ of all deaths) each year [49]. In the elderly, CVD accounts for even more than $80 \%$ of all deaths [50]. High arterial blood pressure is a key determinant of CVD [24] and is the leading attributable factor for developing heart failure [39]. Elevated isolated systolic blood pressure (SBP) is becoming increasingly relevant in the ageing population as $60 \%$ of people aged 60 years or older have elevated SBP (i.e. SBP>140 $\mathrm{mmHg}$ ) [34].

The (age-related) stiffening of arteries has been identified as a key determinant and precursor of elevated SBP $[18,21,28]$. The main contributors to elevated SBP are believed to be decreased (central) arterial compliance and increased systolic wave reflection (i.e. earlier arrival of reflected pressure wave) [28]. Increased myocardial afterload due to elevated SBP affects the way the left ventricle (LV) adapts and becomes hypertrophic [12]. Recently, the ESH/ESC guidelines for the management of hypertension have emphasised the importance of increased arterial stiffness (assessed as pulse wave velocity (PWV)) and LV hypertrophy (LVH) (assessed as LV mass index (LVMI)) in determining overall cardiovascular risk [26].

A pattern of increased arterial stiffness, high blood pressure, and LVH is frequently observed in smaller observational, cross-sectional studies [5, 30, 44]. Due to design and study size, such studies are seriously limited in identifying causality. Furthermore, arterial stiffness measurements are inherently blood pressure-dependent [36], complicating the causative interpretation of observed changes in both arterial stiffness and LV structure and function.

Considering the current pathophysiological paradigm linking arterial stiffening, elevation of SBP, and development of LVH/LV failure [47], our aim was to review the available randomised controlled trials (RCTs) and cohort studies, reporting intervention-induced changes in blood pressure, arterial stiffness and LV structure/function as primary outcomes. We evaluated whether intervention-induced changes in arterial stiffness correlated with improvement in LV structure and function. In our meta-analysis, we included the orders of magnitude of changes in PWV and of changes in LVM, in relation to changes in SBP and DBP.

\subsection{Methods}

\subsubsection{Protocol and registration}

In this study, we applied the Preferred Reporting Items for Systematic reviews and Meta-Analyses (PRISMA) statement for studies evaluating medical interventions [23]. 


\subsubsection{Search strategy and study selection}

We extensively searched the PubMed, EMBASE, Cochrane and Web of Science

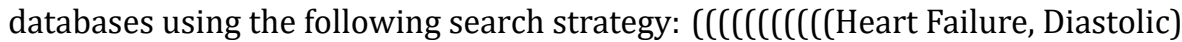
OR Heart Failure, Diastolic) OR diastolic dysfunction) OR isolated systolic hypertension) OR pulse pressure) OR Blood pressure)) AND (C(C(C(Hypertrophy, Left Ventricular) OR Hypertrophy, Left Ventricular) OR left ventricle hypertrophy) OR cardiac hypertrophy) OR cardiomegaly) OR cardiomegaly)) AND (C(C(C(blood pressure) OR blood pressure) OR hypertension) OR high blood pressure) OR hypertension $))$ AND (C(C(((arterial stiffness) OR vascular stiffness) OR vascular stiffness) OR pulse wave velocity) OR pulse wave analysis) OR pulse wave analysis) OR distensibility) $)))$ AND ((((physical activity*) OR motor activity) OR Motor activity) OR (C(C(C((Crevalidation) OR Exercise) OR Exercise) OR Life Style) OR Life Style) OR Therapeutics) OR Therapeutics) OR medical treatment) OR medication))J). The starting date was determined as 1 January 1990 and the search was updated till 20 April 2017. The reference lists and citations of the obtained articles were handsearched for additional searches. Articles were included in this systematic review if they fulfilled the criteria described below (Fig. 2.1):

1. English-written randomised controlled trials (RCTs), prospective observational studies or case-control studies, investigating both an interventionand control group, examining i) the relationship between arterial stiffness and (diastolic) heart failure in isolated systolic hypertension, or ii) the effects of medication, several compounds and lifestyle (diet or exercise) on blood pressure, arterial stiffness and cardiac structure/function, in human follow-up studies (subjects acting as their own controls).

2. Individuals with isolated systolic hypertension and/or heart failure. No restrictions on age were enforced. Both sexes were included.

3. Studies investigating (primary) outcome measures (see summary measures) Studies were excluded a) if populations with kidney haemodialysis/ peritoneal dialysis were examined, b) if the estimated glomerular filtration rate (eGFR) was lower than $30 \mathrm{ml} / \mathrm{min} / 1.73 \mathrm{~m}^{2}, \mathrm{c}$ ) if there were no ventricular or vascular changes reported, or $\mathrm{d}$ ) if the studies were classified as retrospective cohort- and/or cross-sectional studies. Additionally, review articles and case reports were not eligible for inclusion.

\subsubsection{Study selection and data extraction}

When eligibility criteria and the search strategy were realised by three investigators (K.M.W, A.A.K. and K.D.R.), selection screening, based on title and abstract according to the a priori retrieved inclusion- and exclusion criteria, was conducted. In case of mismatch between the investigators, inclusion of an article was based on agreement by consensus during the selection screening. 


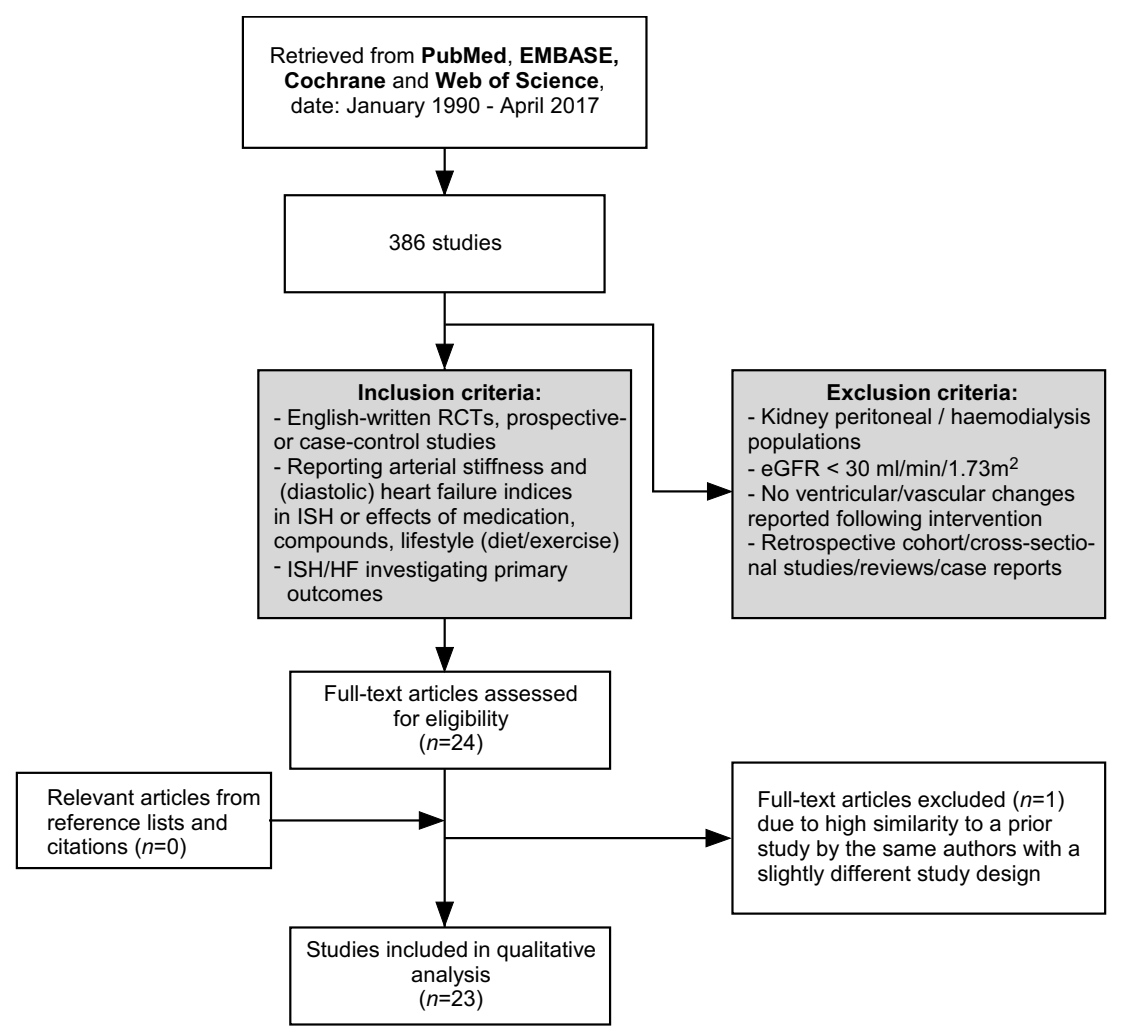

Figure 2.1: Search and selection of studies.

Full-text publications were reviewed by two investigators (K.M.W. and M.H.G.H.) if eligibility criteria were satisfied. Study characteristics, risk of bias within studies and results/conclusions of individual studies were reviewed independently of each other.

From the selected studies, when available, the following data were extracted: i) study methods (design, data collection, time period), ii) participant characteristics (inclusion/exclusion, size, origin, setting), iii) intervention/placebo treatment (type, dose, duration), and iv) outcome measures. In case of a mismatch between first and second reviewer, agreement was achieved by consensus.

\subsubsection{Risk of bias}

Two reviewers (K.M.W. and M.H.G.H.) independently assessed risk of bias to ascertain the validity of the included studies. If present, discrepancies were resolved by agreement based on consensus. Using the online Cochrane handbook for writing systematic reviews [15], sequence generation, allocation concealment, randomisation, blinding of participants and personnel, proportion of drop-outs, 
Table 2.1: Overview of arterial stiffness measured.

\begin{tabular}{cccl}
\hline Name & Abbreviation & Regional or single-point PWV & Studies [ref. no.] \\
\hline Carotid-femoral pulse wave velocity & cfPWV & regional & {$[2,4,13,14,31,42]$} \\
Brachial-ankle pulse wave velocity & baPWV & regional & {$[1,38,41,45]$} \\
Aortic pulse wave velocity & aoPWV & single-point & {$[8,19,32,43]$} \\
\hline
\end{tabular}

similarity between therapies, selective outcome reporting and sponsors influence were assessed for RCTs. Prospective observational studies were assessed by the Newcastle Ottawa Scale [46], in which selection-, comparability- and exposure parameters were rated.

\subsubsection{Summary measures}

Primary outcome measures were changes in i) arterial stiffness and compliance, ii) blood pressure, iii) pulse pressure, iv) LV hypertrophy, LV mass (LVM) and LV mass index (LVMI), (v) diastolic heart failure, and particularly relations between those variables. The included studies report on the one hand regional pulse wave velocities (i.e. cfPWV and baPWV, based on transit time) and on the other hand single-point aortic stiffness. These studies did not utilise carotid measurements. Subsequently, from aortic stiffness estimates, we obtained estimates of aoPWV using the Bramwell-Hill equation [6]. In case compliance (CC) was reported we used PWV $=\left(A_{0} / \rho C C\right)^{1 / 2}$, whereas if distensibility $(D C)$ was reported, we used $\mathrm{PWV}=(1 / \rho \mathrm{DC})^{1 / 2}$ (see ${ }^{*}$, Table 2.A1). Here, $A_{0}$ is the diastolic cross-sectional area, defined $A_{0}=0.25 \pi$ diameter $^{2}$ and $\rho$ blood density, assumed $1050 \mathrm{~kg} / \mathrm{m}^{3}$.

In total, six studies reported cfPWV, four studies reported baPWV and five studies reported aortic distensibility or compliance as arterial stiffness outcome, respectively (Table 2.1). Although pulse pressure was included in our search strategy, we did not include these results in our analysis to avoid it being interpreted as arterial stiffness measure.

\subsubsection{Outcome of interest and statistical analysis}

For each study, we extracted average primary outcomes measures per treatment arm. The outcomes were analysed for homogeneity via statistical analyses. Data were visualised using scatter plots, whereas weighted Pearson's correlation coefficients and linear regression coefficients were calculated to explore between-study associations between changes in PWV and SBP, LVMI and SBP, and LVMI and PWV, respectively.

To internally validate our findings, we performed a sensitivity analysis of the correlation and regression parameters by repeating the statistical analyses after omission of studies not specifically reporting the effect of antihypertensive medication. Additionally, we repeated the statistical analyses using only the data from RCTs. A $p$-value $<0.05$ was considered statistically significant. All statistical analyses were performed using SPSS version 24 (IBM Corp, Armonk, NY, USA). 


\subsection{Results}

\subsubsection{Study selection}

Using our search strategy, we identified 386 potentially relevant articles from PubMed $(n=221)$, EMBASE $(n=1)$, Cochrane $(n=50)$ and Web of Science $(n=114)$. A flow diagram of the study selection process is shown in Fig. 2.1. Eventually, 23 articles with a total of 2573 subjects met the inclusion- and eligibility criteria and were included in this systematic review.

\subsubsection{Risk of bias}

The quality of the different included studies varied. For RCT-studies (Table A1), the domains 'allocation concealment', 'blinding of participants and personnel' and 'blinding of outcome assessment' constitute a plausible risk of bias for most studies, which negatively influenced the outcome for these individual studies, and could lead to high risks of performance bias. On the other hand, most studies demonstrated adequate 'sequence generation', 'follow-up of patients' and 'similarity of therapies'. In addition, low chance of publication bias was indicated, via adequate exclusion of 'undesirable influences of sponsors' and 'selective-free outcome reporting'.

For cohort studies (Table A2), the domains 'selection', 'comparability' and 'exposure' were adequate indicating low risk of bias. Two studies $[33,40]$ constituted overall a significantly higher risk of bias, and therefore, extra attention is needed when interpreting those results.

\subsubsection{Study characteristics}

The characteristics of included studies are summarised in Table 2.2 and Table 2.3 Fourteen studies were qualified as RCTs, while the other nine were prospective observational cohort studies. The follow-up period ranged from three months to 4.8 years for all English-written studies. The number of participants ranged from $n=20$ to $n=873$. Mainly, essential hypertensive patients with or without cardiac and/or vascular alterations were included in studies in single-centre tertiary care centres in European and East Asian countries. The main exclusion criteria were cerebrovascular- and/or renal disorders.

The majority of the trials used antihypertensive drugs, such as angiotensin II receptor blockers (ARBs), angiotensin converting enzyme inhibitors (ACEIs), calcium channel blockers (CCBs), diuretics, and beta-blockers (BBs), while other trials examined the effects of either weight loss, physical training, surgery, treatment with agalsidase beta or high doses of allopurinol. Outcome measures included changes in vascular stiffness, pulse pressure, LVM and LV diastolic function parameters, and correlations between those variables. 


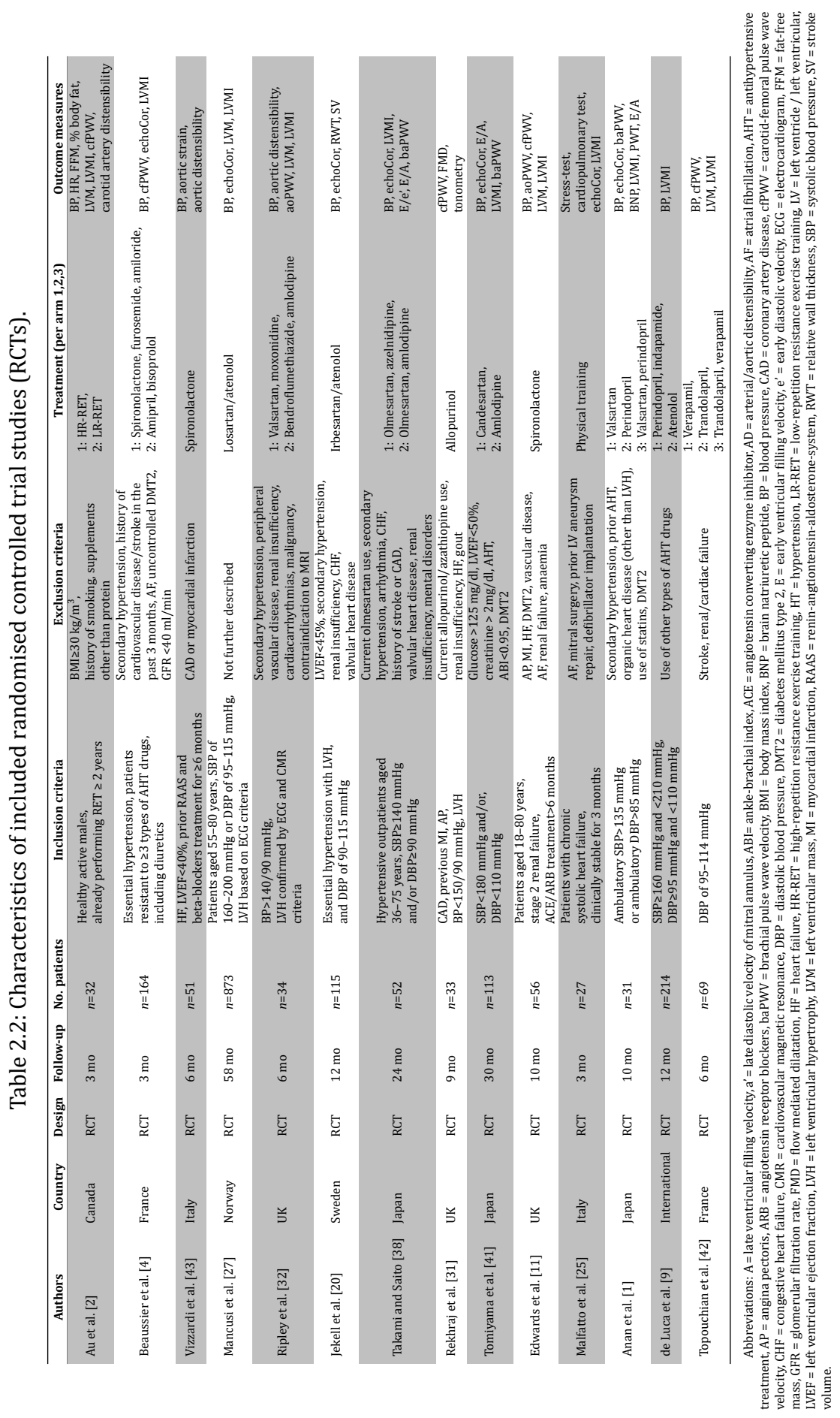




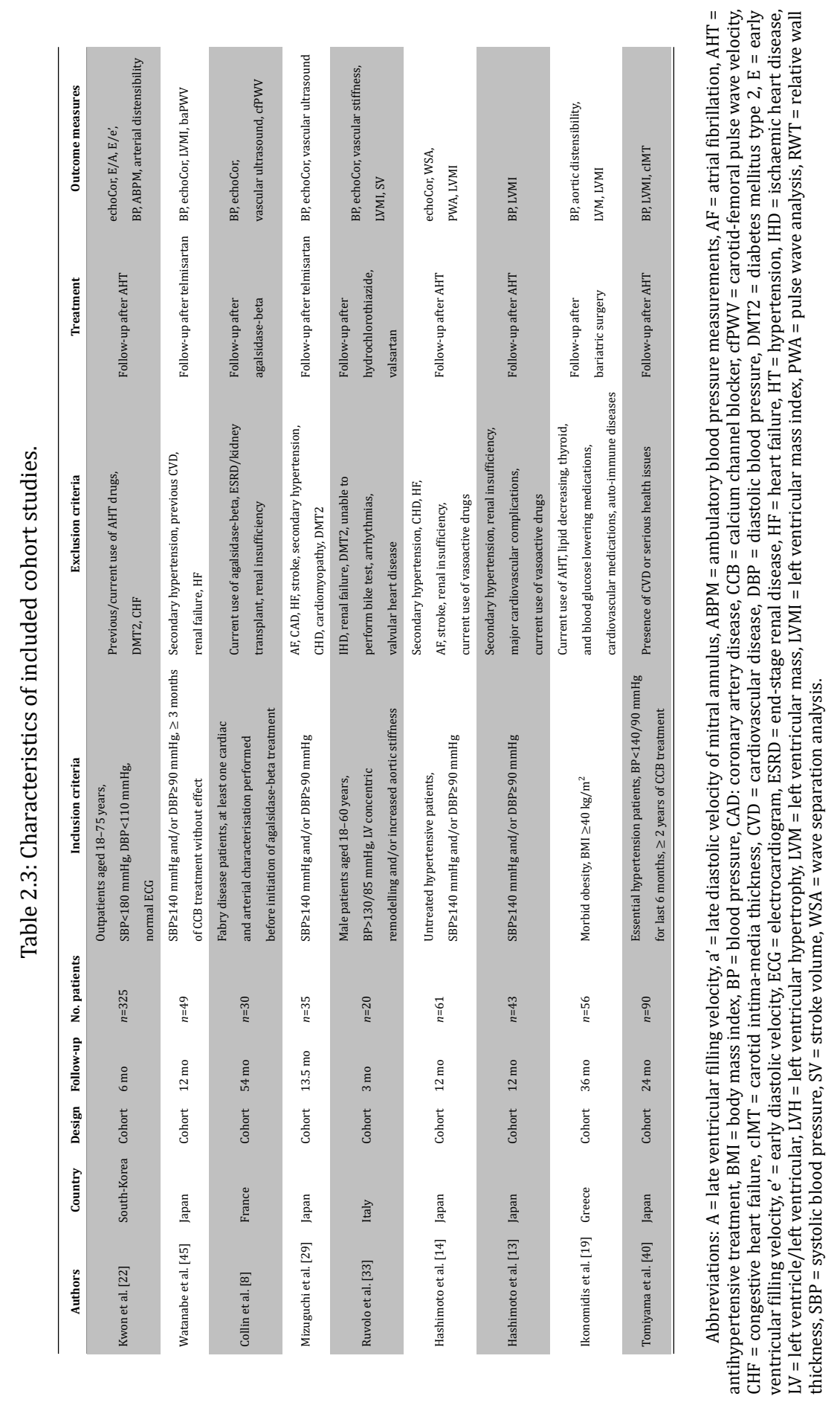




\subsubsection{Studies with vascular and ventricular outcome variables}

A total of 13 studies reported values on changes in SBP, PWV and LVMI after intervention (Fig. 2.2).

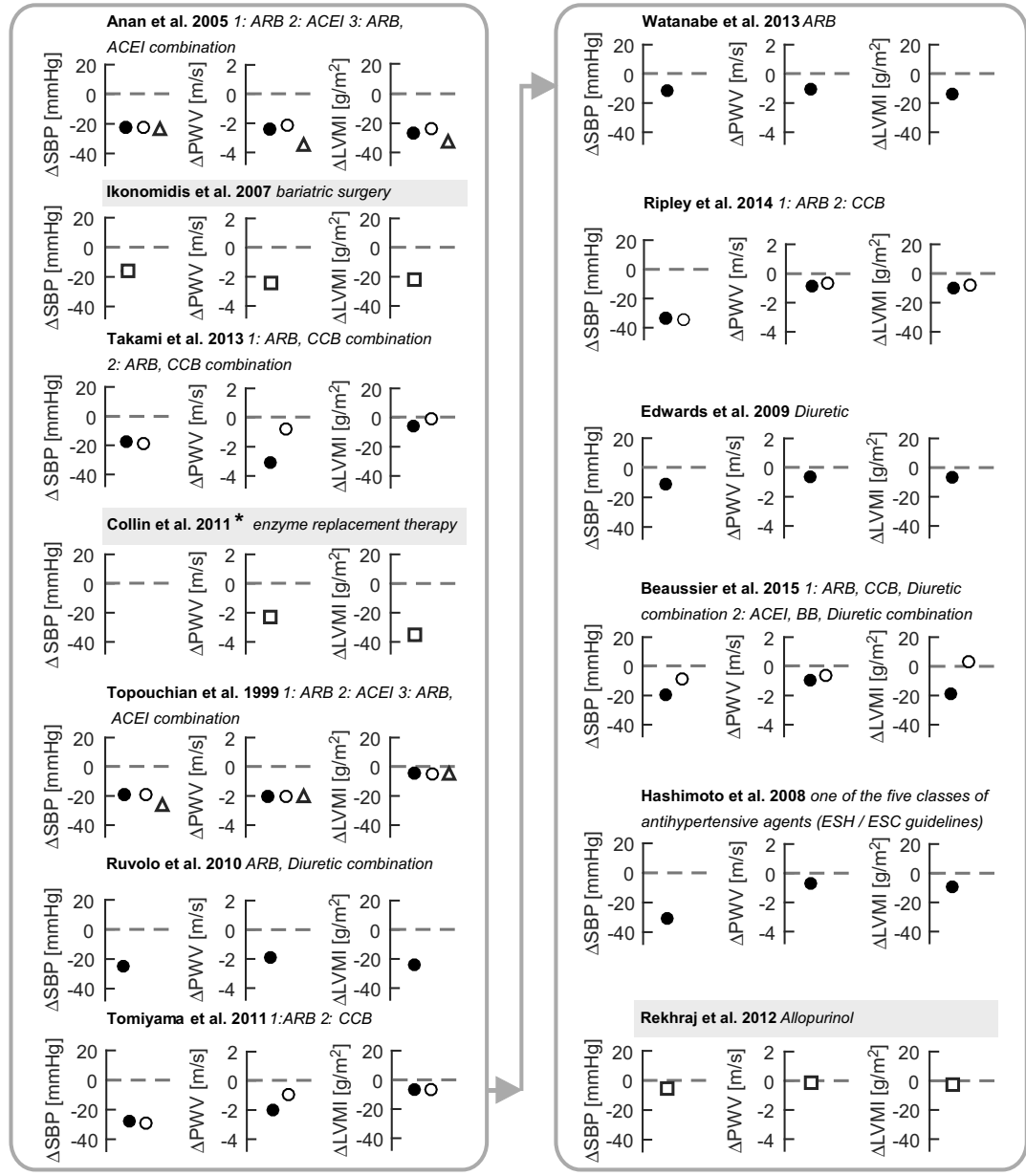

Figure 2.2: Effect of intervention on mean changes in systolic blood pressure ( $\triangle \mathrm{SBP})$, pulse wave velocity $(\triangle \mathrm{PWV})$ and left ventricular mass index $(\triangle \mathrm{LVMI})$. Studies are listed according to magnitude of $\Delta \mathrm{PWV}$, from largest (top-left) to smallest (bottom-right). Ten of the thirteen studies had antihypertensive medication as intervention (circles, triangles), while three (squares) had other interventions, as indicated. Symbols indicate treatment arms (i.e. closed circle $=$ arm 1, open circle $=\operatorname{arm} 2$, triangle $=\operatorname{arm} 3$ ). Abbreviations: ACEI: angiotensin converting enzyme inhibitor, ARB: angiotensin-receptor blockers, BB: beta-blockers, CCB: calcium channel blockers. *No quantitative change in SBP reported but narratively reported as '(Central) systolic blood pressure and pulse pressure were not reduced,.... 
The remaining 10 studies, reporting only one or two of the outcome variables, are not displayed in Fig. 2.2 (data given in Table 2.A1). At study level, changes in SBP seemingly correlated with reductions in both PWV and LVMI. Ripley et al. [32] reported no substantial differences between the change in SBP, PWV and LVMI among treatment arms (Fig. 2.2). Conversely, three studies [1, 38, 41] reported considerably different PWV reductions within treatment arms, despite a similar reduction in SBP. Takami and Saito [38] consisted of two treatment arms comparing two types of ARB, CCB combination treatment, whereas Anan et al. [1] consisted of three treatment arms comparing between ARB and ACEI treatment as well as ARB, ACEI combination treatment. Tomiyama et al. [41] consisted of an ARB treatment arm and a CCB treatment arm, respectively. Furthermore, in studies consisting of two or more treatment arms it appeared that the study arm with the highest reduction in PWV and SBP, consistently corresponded to the treatment arm with the highest reduction in LVMI.

\subsubsection{Blood pressure in relation to vascular- and ventricular changes}

Table 2.4 shows changes in PWV and LVMI stratified by mean changes $(\Delta)$ in SBP and $\mathrm{DBP}$, defined by pressure ranges $\triangle \mathrm{DBP} \geq-7 \mathrm{mmHg}$ versus $\triangle \mathrm{DBP}<-7 \mathrm{mmHg}$, and $\Delta \mathrm{SBP} \geq-13 \mathrm{mmHg}$ versus $\Delta \mathrm{SBP}<-13 \mathrm{mmHg}$, respectively. The choice of the pressure ranges was based on twice the intra-session standard deviation for DBP and SBP, as reported earlier [36]. We found that greater reductions in blood pressure (both SBP as DBP) were indicative for greater PWV reductions, whereas for LVMI reductions this pattern only appear to hold for greater DBP-changes (Table 2.4).

Table 2.4: Blood pressure changes and associated changes in arterial stiffness and ventricular mass index.

\begin{tabular}{lcc}
\hline BP change $[\mathrm{mmHg}]$ & $\Delta \mathrm{PWV}[\mathrm{m} / \mathrm{s}]$ & $\Delta \mathrm{LVMI}\left[\mathrm{g} / \mathrm{m}^{2}\right]$ \\
\hline$\Delta$ DBP $\geq-7$ & $-0.9(-3.2,-0.14)$ & $-7.3(-22,-2.8)$ \\
$\Delta$ DBP $<-7$ & $-2.0(-3.7,-0.6)$ & $-11.0(-35,-3.9)$ \\
$\Delta$ SBP $\geq-13$ & $-0.8(-2.4,-0.14)$ & $-13.0(-24,+4.5)$ \\
$\Delta$ SBP $<13$ & $-2.0(-3.7,-0.7)$ & $-8.0(-35,-2.8)$ \\
\hline
\end{tabular}

Changes are presented as median (minimum, maximum). Abbreviations: $\triangle \mathrm{DBP}$ and $\Delta \mathrm{SBP}$ denote changes in diastolic and systolic blood pressure, whereas $\triangle \mathrm{LVMI}$ and $\Delta \mathrm{PWV}$ denote changes in left ventricular mass index and pulse wave velocity, respectively.

\subsubsection{Pooling of data and correlations between SBP, PWV and LVMI within studies reporting all three variables}

We pooled and plotted $\triangle \mathrm{SBP}, \triangle \mathrm{PWV}$ and $\triangle \mathrm{LVMI}$ data to explore potential associations among these outcome measures (Fig. 2.3). 

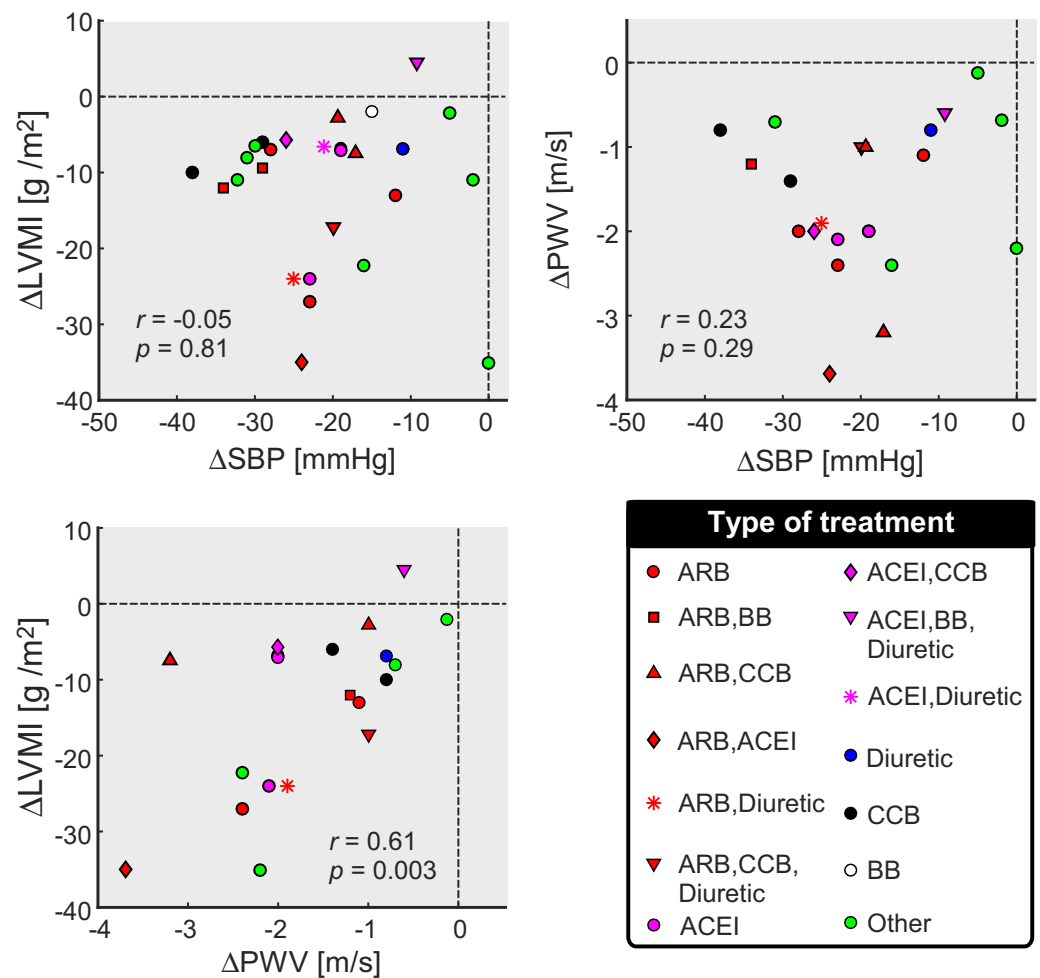

Figure 2.3: Scatter plots of mean changes in left ventricular mass index ( $\Delta$ LVMI), pulse wave velocity $(\triangle \mathrm{PWV})$, and systolic blood pressure $(\triangle \mathrm{SBP})$. Studies with two or more treatment arms were plotted as separate data points. Markers indicate treatment type, i.e. the prescribed antihypertensive drug class or combination of classes, respectively. Abbreviations: ACEI: Angiotensin converting enzyme inhibitor, ARB: Angiotensin-receptor blockers, BB: beta-blockers, CCB: Calciumchannel blockers. Symbols: $r$ : Pearson correlation coefficient. $p$ : statistical significance level.

Regarding SBP, the proportion in reduction did not correlate significantly to the proportion in reductions of the other two outcome measures (i.e. $\triangle \mathrm{PWV}$ vs. $\Delta$ SBP: $r=0.23, p=0.29 ; \Delta$ LVMI vs. $\Delta$ SBP: $r=-0.05, p=0.81$ ). However, we found a significant positive correlation between changes in $\Delta \mathrm{PWV}$ and $\Delta$ LVMI (Fig. 2.3, $r=0.61, p=0.003)$. Furthermore, linear regression of $\Delta \mathrm{PWV}$ on $\Delta$ LVMI suggested a $6.9 \mathrm{~g} / \mathrm{m}^{2}$ decrease in LVMI per $1 \mathrm{~m} / \mathrm{s}$ decrease in PWV $(95 \% \mathrm{CI}=[1.9 ; 11.8]$ $\mathrm{g} / \mathrm{m}^{2} / \mathrm{m} / \mathrm{s}$ ).

The analyses were repeated following omission of the non-antihypertensive treatment studies $[2,8,19,25]$. Briefly, this caused only minor changes in the correlation and regression parameters $\left(r=0.57, p=0.013\right.$, and $B=6.5 \mathrm{~g} / \mathrm{m}^{2} / \mathrm{m} / \mathrm{s}$, $95 \% \mathrm{CI}=[1.6 ; 11.4] \mathrm{g} / \mathrm{m}^{2} / \mathrm{m} / \mathrm{s}$, respectively). Similarly, we found minor changes 
in the correlation and regression parameters when using only data from RCTs $\left(r=0.62, p=0.010\right.$, and $\left.B=6.6 \mathrm{~g} / \mathrm{m}^{2} / \mathrm{m} / \mathrm{s}, 95 \% \mathrm{CI}=[1.9 ; 11.4] \mathrm{g} / \mathrm{m}^{2} / \mathrm{m} / \mathrm{s}\right)$. Given the limited amount of data points available for regression analysis (Fig. 2.3), we chose not to evaluate quadratic instead of linear associations, since this would result in overfitting. We explored possible mutual relationships between types of $\triangle \mathrm{PWV}$ (i.e. as assessed by cfPWV, baPWV and aoPWV) with both $\triangle \mathrm{SBP}$ and $\Delta$ LVMI (Fig. A1).

Our analysis did not demonstrate statistically significant associations. Nevertheless, in particular $\Delta$ baPWV and $\triangle$ aoPWV, but not $\Delta \mathrm{cfPWV}$, showed trends with $\Delta$ LVMI $(r=0.62, p=0.10 ; r=0.83, p=0.08 ; r=0.26, p=0.54$, respectively $)$. Furthermore, $\Delta \mathrm{cfPWV}$ and $\triangle \mathrm{aoPWV}$, but not $\triangle$ baPWV showed trends with $\Delta \mathrm{SBP}(r=0.59$, $p=0.07 ; r=-0.85, p=0.07 ; r=0.14, p=0.74$, respectively), indicating that a larger power (in future studies) might possibly lead to significant correlations. In the above analyses a limited amount of data points was available (Fig. A1).

\subsection{Discussion}

\subsubsection{Key findings}

Our systematic review of well-controlled clinical intervention studies provides evidence that a decrease in arterial stiffness could contribute to the reduction of LVM in (hypertensive) patients. However, we could not obtain convincing evidence supporting the causative pathophysiological [arterial stiffening - elevated SBP — LV hypertrophy/ failure]-chain.

A major complication is that arterial stiffness measurements such as PWV are inherently blood pressure-dependent [36, 37]. In the present review, we found studies showing a significant decrease in PWV in excess of $\approx 0.5 \mathrm{~m} / \mathrm{s}$, which is typically the measurement variability order of magnitude, and in excess of 1 $\mathrm{m} / \mathrm{s}$, which our group identified as the change in PWV expected for a change in diastolic blood pressure of $10 \mathrm{mmHg}$ [36]. As such, some of the observed significant changes in PWV may partially or fully be attributable to a change in blood pressure, without a change in intrinsic arterial stiffness.

We did not find a study that was appropriately designed to disentangle pressureindependent arterial stiffness effects from plain blood pressure lowering, nor are we aware of a study that extensively described SBP, PWV and LVMI changes over many time-points. We expect that changes in PWV precede changes in LV mass. There were several studies included reporting arterial stiffness and ventricular structure at multiple time-points $[8,19,38,41]$. However, only data from Collin et al. [8] suggested that a change in PWV could precede a change in LV mass. Despite the present analysis, therefore, it remains cumbersome to assess the direct effect of antihypertensive treatment on LV structure and function as well as test the hypothesised causal relation between increased arterial stiffness and LVH. 


\subsubsection{Clinical implications and future work}

Our results indicate a significant positive correlation between changes in arterial stiffness and LVM. Only two of the 23 included studies [22, 38] reported changes in $\mathrm{LV}$ diastolic function indices (i.e. the outcome variables $\mathrm{E} / \mathrm{A}$ and $\mathrm{E} / \mathrm{e}^{\prime}$, respectively). Those studies found independent statistical associations between on the one hand reductions in PWV and pulse wave reflection magnitude (assessed by means of augmentation index), and on the other hand improvements in LV diastolic function (via LVMI reduction and improvements in E/A and E/e', Table 2.A1). Takami and Saito [38] hypothesised that the mechanism involved could be a delay in arrival of the reflected pulse wave due to decreased PWV, which in turn reduces left ventricular afterload [38]. However, it should be noted that using augmentation index as proxy for wave reflection magnitude is deemed contentious by some researchers in the field [3, 17]. Hashimoto et al. [14] reported a positive correlation between changes in LVM and reflection magnitude, calculated using a 'gold standard' method of assessing pulse wave reflections (i.e. based on solving the physical laws of mass conservation and momentum balance [47]. The authors reported that reducing stiffness of peripheral muscular arteries, which they considered to be the root cause of increased pulse wave reflection, was more important than reducing central arterial stiffness, in the regression of LVM [14]. Tomiyama et al. [41] reported that candesartan leads to significantly greater reductions in stiffness of muscular arteries (i.e. assessed by brachial-ankle PWV), as compared to amlodipine [41], with similar decreases in LVMI of $7 \mathrm{~g} / \mathrm{m}^{2}$ and 6 $\mathrm{g} / \mathrm{m}^{2}$, respectively.

In summary, our results suggest that therapeutic agents that aim to lower arterial stiffness may lead to greater reductions in LVM. However, further clinical trials with multiple follow-up measurements, using pressure-independent arterial stiffness indices, are required to establish the causative role of arterial stiffnesslowering in reduction of LVM. To achieve the latter, a stiffness index such as $\mathrm{CAVI}_{0}$ could be a promising candidate [37]. Additionally, directing more attention to pulse wave reflection indices may further advance insight into LVM reduction $[14,38]$. Of particular interest are (lifestyle or surgical) interventions improving physical activity and diet, since these kinds of trials provided promising results in terms of arterial stiffness and LV diastolic function improvements $[2,8,19,25,31]$, without invoking per se the effects of direct actions of antihypertensive drugs on the cardiovascular system.

\subsubsection{Limitations}

Our review is limited by its reliance on published data causing an inherent risk of publication bias, as neutral studies (without changes in LVMI or PWV) are less likely to be accepted by publishers.

A general conclusion about the effect size of lowering blood pressure and arterial stiffness on LVH is difficult, since the quality of the studies was variable (quality rating between 2 and 8 points out of 10 for RCTs and between 5 and 9 points 
for cohort studies, Table A1 and A2). Most studies quantified arterial stiffness using carotid-femoral PWV or brachial-ankle PWV (i.e. calculated using pulse transit time and path length). For studies reporting either a change in compliance or distensibility, we were limited to calculating an estimate of PWV using the Bramwell-Hill equation. Regional (i.e. carotid-femoral and brachial-ankle) PWV values are physically different compared to single-point (i.e. aortic) PWV values, calculated using the Bramwell-Hill equation. However, previous work including patient studies $[10,16]$ and mechanistic computational studies [48] showed reasonable proportionality between regional PWV and single-point PWV. Also, the study of Chow and Rabkin [7] showed appropriate proportionality between baPWV and cfPWV. Therefore, we believe that pooling of changes in cfPWV, baPWV, and aoPWV can be justified. We, however, cannot exclude that pooling the various methodologies of PWV assessment influenced the associations we found.

Furthermore, considering the heterogeneity in antihypertensive treatment and the limited number of studies included, we have not been able to conduct antihypertensive drug class specific analyses. Lack of correlation between changes in SBP and LVMI could be related to the fact that most studies included in this review performed office BP measurements instead of 24-hour ambulatory BP measurements. Office blood pressure measurements contain more variable SBP readings, e.g. due to inter-individual differences in white-coat effect. Previously, it was reported that in hypertensive children 24-hour SBP relates with LVMI, but not with office SBP [35].

Lastly, minor limitations of this review include our language restriction for only English written articles, and our inclusion of non-RCT studies, which in the hierarchy of evidence are inferior to RCT studies.

\subsection{Conclusions}

This systematic review demonstrates that there is evidence in well-controlled clinical intervention studies that a decrease in arterial stiffness is associated with reduction of LV mass. To eliminate blood pressure-dependent effects for the correlation between arterial stiffness and LV structure, blood pressure-independent markers should be used in future studies. In that way, better identification of potential targets for antihypertensive drug treatment may be facilitated. In addition, the potential of lifestyle interventions (e.g. physical activity and diet) in the research field remains to be emphasised. 


\section{A1 Appendix Figures and Tables}
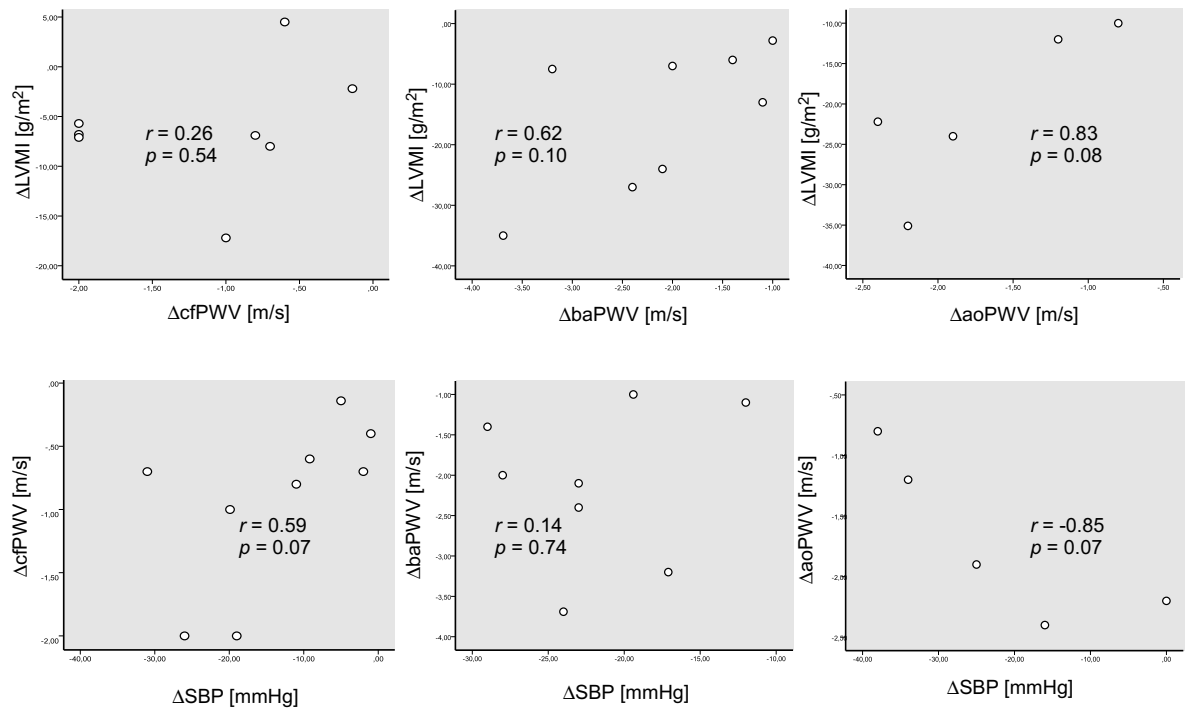

Figure A1: Top: Scatter plots displaying the relation between changes in left ventricular mass index $(\triangle \mathrm{LVMI})$, and changes in carotid-femoral, brachial-ankle, and aortic pulse wave velocity ( $\Delta$ cfPWV, $\Delta$ baPWV, $\triangle$ aoPWV, respectively). Bottom: Relation between changes in $\Delta \mathrm{cfPWV}, \Delta \mathrm{baPWV}, \Delta \mathrm{aoPWV}$ and changes in systolic blood pressure $(\Delta \mathrm{SBP})$. Symbols: $r$ : Pearson correlation coefficient. $p$ : statistical significance level. 

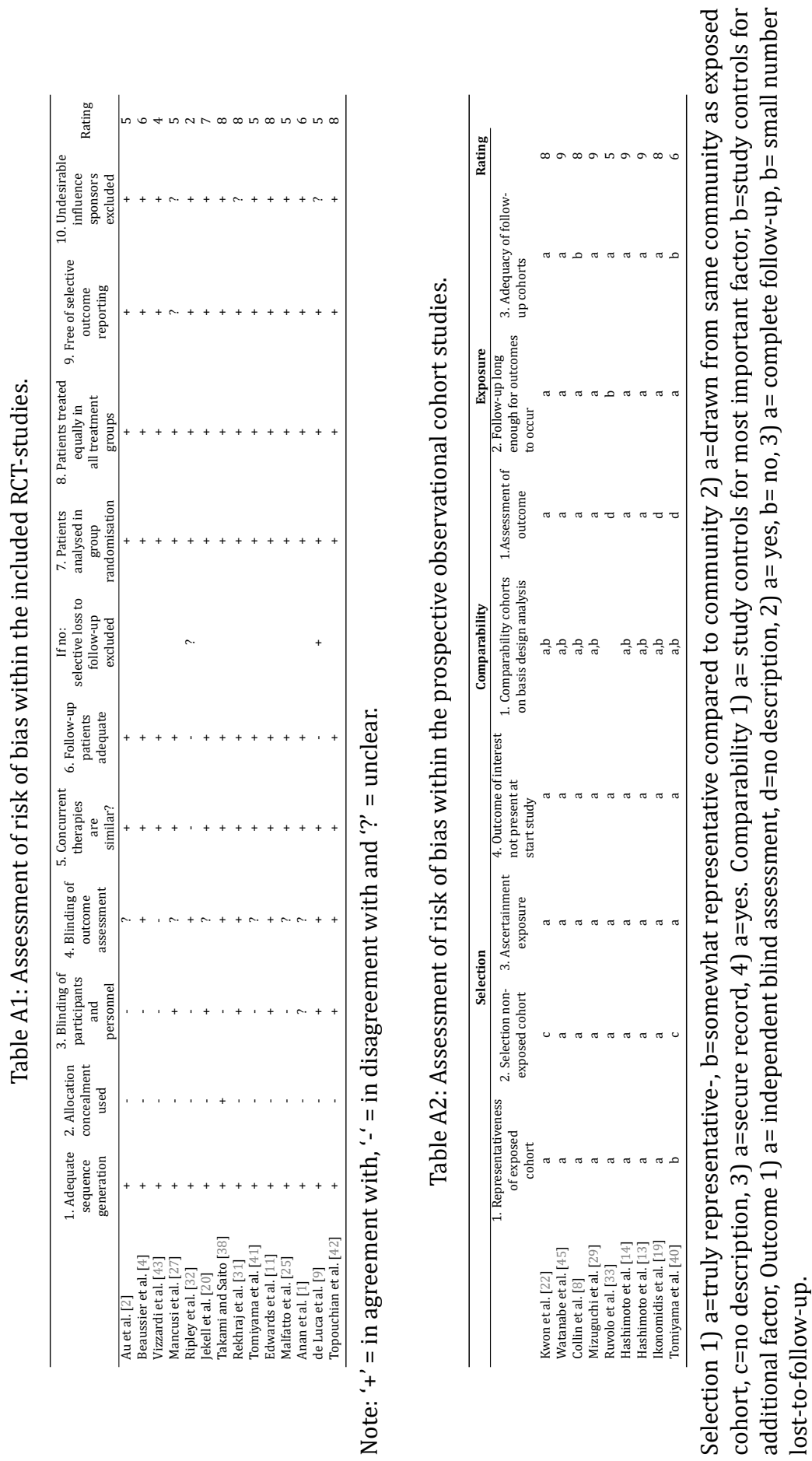


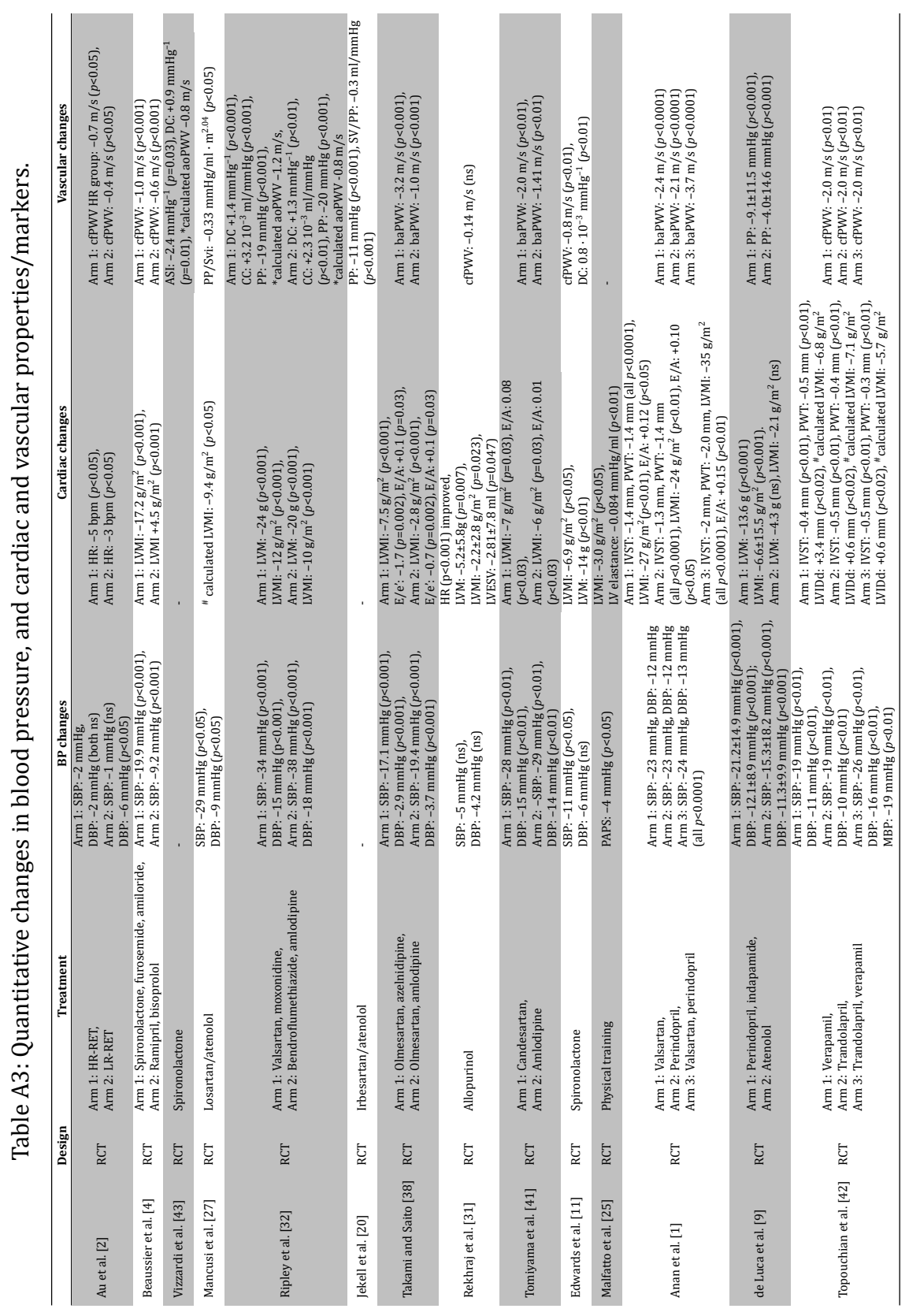




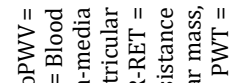

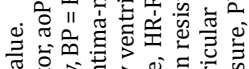

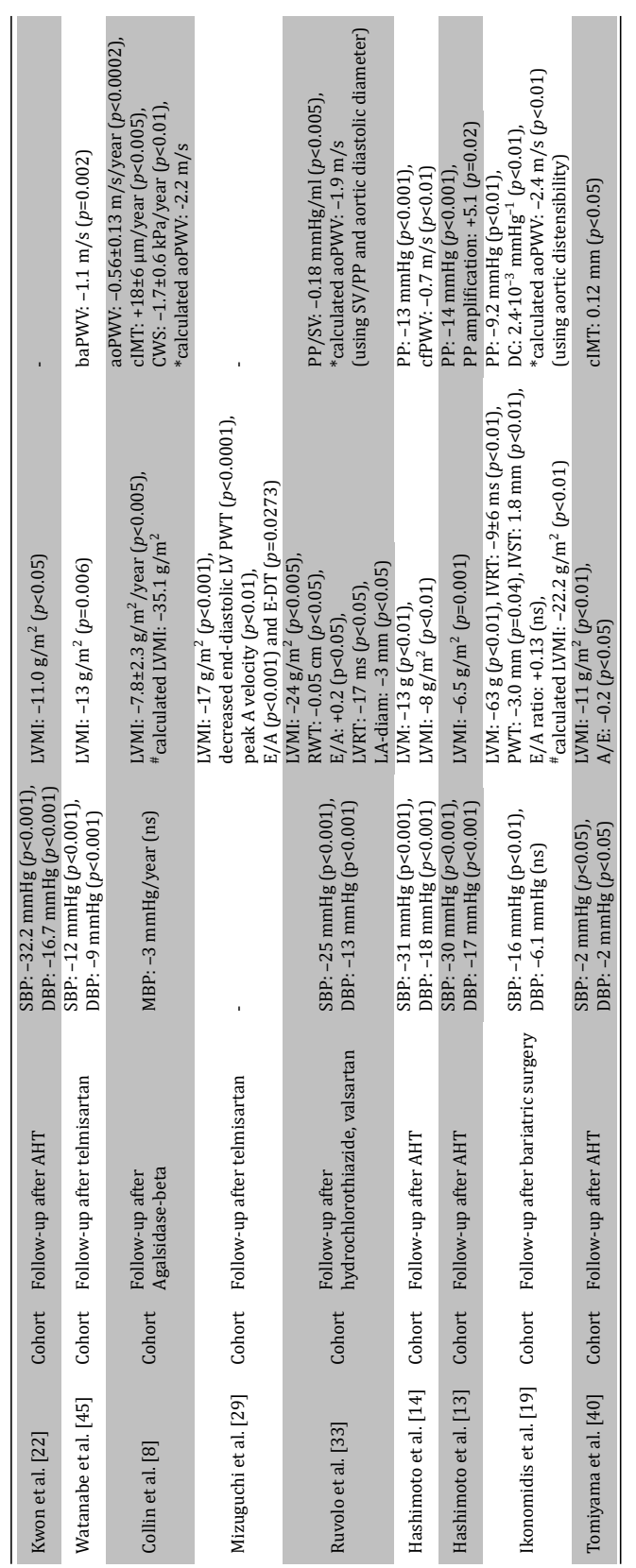

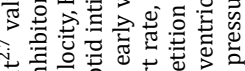
茄恕范

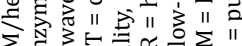
$\sum_{0} \int_{0} \sum_{0}$

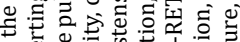
छ

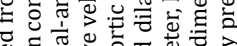



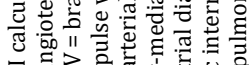

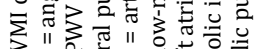

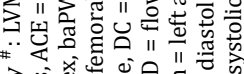

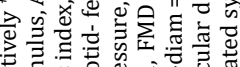

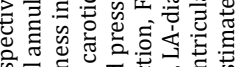
कृत्र

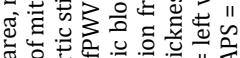

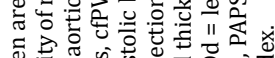

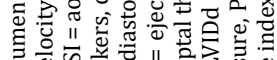
要递要

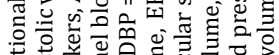

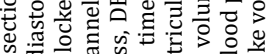

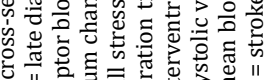
“" 3.

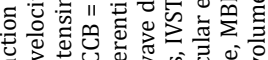

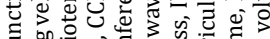

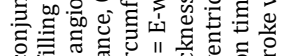

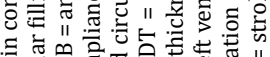

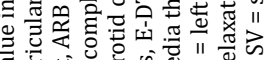

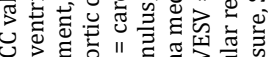

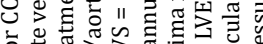
t。

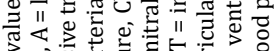

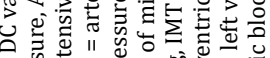

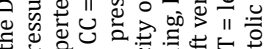

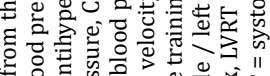

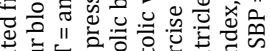

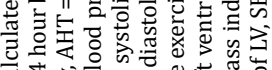


3"1

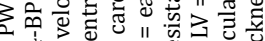

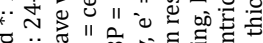

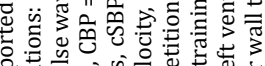

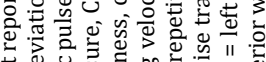

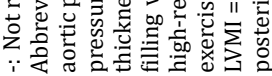





\section{References}

[1] F. Anan, N. Takahashi, T. Ooie, K. Yufu, M. Hara, M. Nakagawa, H. Yonemochi, T. Saikawa, and H. Yoshimatsu. Effects of valsartan and perindopril combination therapy on left ventricular hypertrophy and aortic arterial stiffness in patients with essential hypertension. European Journal of Clinical Pharmacology, 61(5-6):353-9, 2005.

[2] J. S. Au, S. Y. Oikawa, R. W. Morton, M. J. Macdonald, and S. M. Phillips. Arterial stiffness is reduced regardless of resistance training load in young men. Medicine and Science in Sports and Exercise, $49(2): 342-348,2017$.

[3] A. J. Baksi, T. A. Treibel, J. E. Davies, N. Hadjiloizou, R. A. Foale, K. H. Parker, D. P. Francis, J. Mayet, and A. D. Hughes. A meta-analysis of the mechanism of blood pressure change with aging. Journal of the American College of Cardiology, 54(22):2087-92, 2009.

[4] H. Beaussier, P. Boutouyrie, G. Bobrie, M. Frank, S. Laurent, F. Coudore, and M. Azizi. True antihypertensive efficacy of sequential nephron blockade in patients with resistant hypertension and confirmed medication adherence. Journal of Hypertension, 33(12):2526-33, 2015.

[5] P. Boutouyrie, S. Laurent, X. Girerd, A. Benetos, P. Lacolley, E. Abergel, and M. Safar. Common carotid artery stiffness and patterns of left ventricular hypertrophy in hypertensive patients. Hypertension, 25(4 Pt 1):651-9, 1995.

[6] J. C. Bramwell and A. V. Hill. Velocity of transmission of the pulse-wave: and elasticity of arteries. The Lancet, 199(5149):891-892, 1922.

[7] B. Chow and S. W. Rabkin. Brachial-ankle pulse wave velocity is the only index of arterial stiffness that correlates with a mitral valve indices of diastolic dysfunction, but no index correlates with left atrial size. Cardiology Research and Practice, (986847), 2013.

[8] C. Collin, M. Briet, T. C. Tran, H. Beaussier, K. Benistan, M. Bensalah, E. Mousseaux, M. Froissart, E. Bozec, S. Laurent, P. Boutouyrie, and D. P. Germain. Long-term changes in arterial structure and function and left ventricular geometry after enzyme replacement therapy in patients affected with Fabry disease. European Journal of Preventive Cardiology, 19(1):43-54, 2012.

[9] N. de Luca, J. M. Mallion, M. F. O’Rourke, E. O’Brien, K. H. Rahn, B. Trimarco, R. Romero, P. W. De Leeuw, G. Hitzenberger, E. Battegay, D. Duprez, P. Sever, and M. E. Safar. Regression of left ventricular mass in hypertensive patients treated with perindopril/indapamide as a first-line combination: the REASON echocardiography study. American Journal of Hypertension, 17(8): $660-7,2004$.

[10] A. Dogui, N. Kachenoura, F. Frouin, M. Lefort, A. De Cesare, E. Mousseaux, and A. Herment. Consistency of aortic distensibility and pulse wave velocity estimates with respect to the Bramwell-Hill theoretical model: a cardiovascular magnetic resonance study. Journal of Cardiovascular Magnetic Resonance, 13(1):11, 2011.

[11] N. C. Edwards, R. P. Steeds, P. M. Stewart, C. J. Ferro, and J. N. Townend. Effect of spironolactone on left ventricular mass and aortic stiffness in early-stage chronic kidney disease: a randomized controlled trial. Journal of the American College of Cardiology, 54(6):505-12, 2009.

[12] A. Ganau, R. B. Devereux, M. J. Roman, G. de Simone, T. G. Pickering, P. S. Saba, P. Vargiu, I. Simongini, and J. H. Laragh. Patterns of left ventricular hypertrophy and geometric remodeling in essential hypertension. Journal of the American College of Cardiology, 19(7):1550-8, 1992. 
[13] J. Hashimoto, Y. Imai, and M. F. O’Rourke. Monitoring of antihypertensive therapy for reduction in left ventricular mass. American Journal of Hypertension, 20(11):1229-33, 2007.

[14] J. Hashimoto, B. E. Westerhof, N. Westerhof, Y. Imai, and M. F. O’Rourke. Different role of wave reflection magnitude and timing on left ventricular mass reduction during antihypertensive treatment. Journal of Hypertension, 26(5):1017-24, 2008.

[15] G. S. Higgins J P T. Cochrane handbook for systematic reviews of interventions version 5.1.0 [updated march 2011]. The Cochrane Collaboration, 2011. Available from www. cochrane-handbook. org. 2011

[16] C. Huang, D. Guo, F. Lan, H. Zhang, and J. Luo. Noninvasive measurement of regional pulse wave velocity in human ascending aorta with ultrasound imaging: an in-vivo feasibility study. Journal of hypertension, 34(10):2026-2037, 2016.

[17] A. D. Hughes, C. Park, J. Davies, D. Francis, G. T. S. A. Mc, J. Mayet, and K. H. Parker. Limitations of augmentation index in the assessment of wave reflection in normotensive healthy individuals. PLOS ONE, 8(3):e59371, 2013.

[18] J. D. Humphrey, D. G. Harrison, C. A. Figueroa, P. Lacolley, and S. Laurent. Central artery stiffness in hypertension and aging: A problem with cause and consequence. Circulation Research, 118 (3):379-81, 2016.

[19] I. Ikonomidis, A. Mazarakis, C. Papadopoulos, N. Patsouras, F. Kalfarentzos, J. Lekakis, D. T. Kremastinos, and D. Alexopoulos. Weight loss after bariatric surgery improves aortic elastic properties and left ventricular function in individuals with morbid obesity: a 3-year follow-up study. Journal of Hypertension, 25(2):439-47, 2007.

[20] A. Jekell, K. Malmqvist, N. H. Wallen, D. Mortsell, and T. Kahan. Markers of inflammation, endothelial activation, and arterial stiffness in hypertensive heart disease and the effects of treatment: results from the SILVHIA study. Journal of Cardiovascular Pharmacology, 62(6):559-66, 2013.

[21] B. M. Kaess, J. Rong, M. G. Larson, N. M. Hamburg, J. A. Vita, D. Levy, E. J. Benjamin, R. S. Vasan, and G. F. Mitchell. Aortic stiffness, blood pressure progression, and incident hypertension. JAMA, 308(9):875-81, 2012.

[22] B. J. Kwon, S. H. Lee, C. S. Park, D. B. Kim, H. J. Park, S. W. Jang, S. H. Ihm, H. J. Youn, K. B. Seung, and H. Y. Kim. Left ventricular diastolic dyssynchrony in patients with treatment-naive hypertension and the effects of antihypertensive therapy. Journal of Hypertension, 33(2):354-65, 2015.

[23] A. Liberati, D. G. Altman, J. Tetzlaff, C. Mulrow, P. C. Gotzsche, J. P. Ioannidis, M. Clarke, P. J. Devereaux, J. Kleijnen, and D. Moher. The PRISMA statement for reporting systematic reviews and meta-analyses of studies that evaluate healthcare interventions: explanation and elaboration. $B M J, 339: \mathrm{b} 2700,2009$.

[24] S. S. Lim, T. Vos, A. D. Flaxman, G. Danaei, K. Shibuya, H. Adair-Rohani, M. A. AlMazroa, M. Amann, H. R. Anderson, K. G. Andrews, et al. A comparative risk assessment of burden of disease and injury attributable to 67 risk factors and risk factor clusters in 21 regions, 1990-2010: a systematic analysis for the global burden of disease study 2010. The Lancet, 380(9859):2224-2260, 2012.

[25] G. Malfatto, G. Branzi, G. Osculati, P. Valli, P. Cuoccio, F. Ciambellotti, G. Parati, and M. Facchini. Improvement in left ventricular diastolic stiffness induced by physical training in patients with dilated cardiomyopathy. Journal of Cardiac Failure, 15(4):327-33, 2009.

[26] G. Mancia, R. Fagard, K. Narkiewicz, J. Redon, A. Zanchetti, M. Bohm, T. Christiaens, R. Cifkova, G. De Backer, A. Dominiczak, M. Galderisi, D. E. Grobbee, T. Jaarsma, P. Kirchhof, S. E. Kjeldsen, S. Laurent, A. J. Manolis, P. M. Nilsson, L. M. Ruilope, R. E. Schmieder, P. A. Sirnes, P. Sleight, M. Viigimaa, B. Waeber, and F. Zannad. 2013 ESH/ESC guidelines for the management of arterial 
hypertension: the Task Force for the management of arterial hypertension of the European Society of Hypertension (ESH) and of the European Society of Cardiology (ESC). Journal of Hypertension, 31(7):1281-357, 2013.

[27] C. Mancusi, E. Gerdts, G. De Simone, Y. M. Abdelhai, M. T. Lonnebakken, K. Boman, K. Wachtell, B. Dahlof, and R. B. Devereux. Impact of isolated systolic hypertension on normalization of left ventricular structure during antihypertensive treatment (the LIFE study). Blood Pressure, 23(4): 206-12, 2014

[28] G. F. Mitchell, S. J. Hwang, R. S. Vasan, M. G. Larson, M. J. Pencina, N. M. Hamburg, J. A. Vita, D. Levy, and E. J. Benjamin. Arterial stiffness and cardiovascular events: the Framingham Heart Study. Circulation, 121(4):505-11, 2010.

[29] Y. Mizuguchi, Y. Oishi, H. Miyoshi, A. Iuchi, N. Nagase, and T. Oki. Telmisartan improves morphologic and functional changes in both left ventricular myocardium and carotid arterial wall in patients with hypertension: assessment by tissue doppler imaging and carotid ultrasonography. Echocardiography, 27(7):864-72, 2010

[30] A. Pandey, H. Golwala, H. Xu, A. D. DeVore, R. Matsouaka, M. Pencina, D. J. Kumbhani, A. F. Hernandez, D. L. Bhatt, P. A. Heidenreich, C. W. Yancy, J. A. de Lemos, and G. C. Fonarow. Association of 30-day readmission metric for heart failure under the hospital readmissions reduction program with quality of care and outcomes. Journal of the American College of Cardiology: Heart Failure, 4 (12):935-946, 2016.

[31] S. Rekhraj, S. J. Gandy, B. R. Szwejkowski, M. A. Nadir, A. Noman, J. G. Houston, C. C. Lang, J. George, and A. D. Struthers. High-dose allopurinol reduces left ventricular mass in patients with ischemic heart disease. Journal of the American College of Cardiology, 61(9):926-32, 2013.

[32] D. P. Ripley, K. Negrou, J. J. Oliver, G. Worthy, A. D. Struthers, S. Plein, and J. P. Greenwood. Aortic remodelling following the treatment and regression of hypertensive left ventricular hypertrophy: a cardiovascular magnetic resonance study. Clinical and Experimental Hypertension, 37(4): 308-16, 2015.

[33] A. Ruvolo, V. Mercurio, V. Fazio, G. Carlomagno, T. Russo, F. Affuso, and S. Fazio. Efficacy and safety of valsartan plus hydroclorothiazide for high blood pressure. World Journal of Cardiology, 2(5):125-30, 2010.

[34] H. Smulyan, S. Mookherjee, and M. E. Safar. The two faces of hypertension: role of aortic stiffness. Journal of the American Society of Hypertension, 10(2):175-183, 2016.

[35] J. M. Sorof, G. Cardwell, K. Franco, and R. J. Portman. Ambulatory blood pressure and left ventricular mass index in hypertensive children. Hypertension, 39(4):903-908, 2002.

[36] B. Spronck, M. H. G. Heusinkveld, F. H. Vanmolkot, J. Op 't Roodt, E. Hermeling, T. Delhaas, A. A. Kroon, and K. D. Reesink. Pressure-dependence of arterial stiffness: potential clinical implications. Journal of Hypertension, 33(2):330-8, 2015.

[37] B. Spronck, A. P. Avolio, I. Tan, M. Butlin, K. D. Reesink, and T. Delhaas. Arterial stiffness index beta and cardio-ankle vascular index inherently depend on blood pressure but can be readily corrected. Journal of Hypertension, 35(1):98-104, 2017.

[38] T. Takami and Y. Saito. Azelnidipine plus olmesartan versus amlodipine plus olmesartan on arterial stiffness and cardiac function in hypertensive patients: a randomized trial. Drug Design, Development and Therapy, 7:175-83, 2013.

[39] G. Tocci, S. Sciarretta, and M. Volpe. Development of heart failure in recent hypertension trials. Journal of Hypertension, 26(7):1477-86, 2008. 
[40] H. Tomiyama, Y. Kimura, Y. Sakuma, K. Matuno, H. Yoshida, and N. Doba. The effects of monotherapy or combined therapy with an angiotensin converting enzyme inhibitor following initial treatment with calcium channel blockers on residual cardiovascular abnormalities. Clinical and Experimental Hypertension, 22(5):493-506, 2000.

[41] H. Tomiyama, M. Yoshida, J. Yamada, C. Matsumoto, M. Odaira, K. Shiina, and A. Yamashina. Arterial-cardiac destiffening following long-term antihypertensive treatment. American Journal of Hypertension, 24(10):1080-6, 2011.

[42] J. Topouchian, R. Asmar, F. Sayegh, A. Rudnicki, A. Benetos, A. M. Bacri, and M. E. Safar. Changes in arterial structure and function under trandolapril-verapamil combination in hypertension. Stroke, 30(5):1056-64, 1999.

[43] E. Vizzardi, P. D. Pina, G. Caretta, I. Bonadei, E. Sciatti, C. Lombardi, A. D’Aloia, A. Curnis, and M. Metra. The effect of aldosterone-antagonist therapy on aortic elastic properties in patients with nonischemic dilated cardiomyopathy. Journal of Cardiovascular Medicine, 16(9):597-602, 2015.

[44] O. Vriz, J. Magne, C. Driussi, G. Brosolo, F. Ferrara, P. Palatini, V. Aboyans, and E. Bossone. Comparison of arterial stiffness/compliance in the ascending aorta and common carotid artery in healthy subjects and its impact on left ventricular structure and function. International Journal of Cardiovascular Imaging, 33(4):521-531, 2017.

[45] Y. Watanabe, T. Kikuchi, T. Mitsuhashi, H. Kimura, Y. Tsuchida, and K. Otsuka. Administration of angiotensin receptor ii blockade improves vascular function, urinary albumin excretion, and left ventricular hypertrophy in low-risk essential hypertensive patients receiving antihypertensive treatment with calcium channel blockers. Clinical and Experimental Hypertension, 35(2):87-94, 2013.

[46] G. Wells, B. Shea, D. O'Connell, J. Peterson, V. Welch, M. Losos, and P. Tugwell. The NewcastleOttawa scale (NOS) for assessing the quality of nonrandomised studies in meta-analyses. the Ottawa Hospital/l'Hôpital d'Ottawa, 2014.

[47] N. Westerhof and M. F. O’Rourke. Haemodynamic basis for the development of left ventricular failure in systolic hypertension and for its logical therapy. Journal of Hypertension, 13(9):943-52, 1995.

[48] M. Willemet, S. Vennin, and J. Alastruey. Computational assessment of hemodynamics-based diagnostic tools using a database of virtual subjects: Application to three case studies. Journal of Biomechanics, 49(16):3908-3914, 2016.

[49] World Health Organization. About cardiovascular diseases, 2018. http://www. who.int/ cardiovascular_diseases/en/ [accessed 1 March 2018].

[50] A. Yazdanyar and A. B. Newman. The burden of cardiovascular disease in the elderly: morbidity, mortality, and costs. Clinics in Geriatric Medicine, 25(4):563-577, 2009. 


\section{Large vessels as a tree of transmission lines incorporated in the CircAdapt whole-heart model: A computational tool to examine heart-vessel interaction}

The contents of this chapter are based on:

Maarten H.G. Heusinkveld, ${ }^{1}$ Wouter Huberts,,${ }^{1,2}$ Joost Lumens, ${ }^{1}$ Theo Arts, ${ }^{1}$ Tammo Delhaas, ${ }^{1}$ Koen D. Reesink ${ }^{1}$

Large vessels as a tree of transmission lines incorporated in the CircAdapt wholeheart model: A computational tool to examine heart-vessel interaction. PLOS Computational Biology (2019) 15(7): e1007173

\footnotetext{
${ }^{1}$ Department of Biomedical Engineering, CARIM School for Cardiovascular Diseases, Maastricht University, Maastricht, The Netherlands.

${ }^{2}$ Department of Biomedical Engineering, Eindhoven University of Technology, Eindhoven, The Netherlands.
} 


\subsection{Abstract}

We developed a whole-circulation computational model by integrating a transmission line (TL) model describing vascular wave transmission into the established CircAdapt platform of whole-heart mechanics. In the present paper, we verify the numerical framework of our TL model by benchmark comparison to a previously validated pulse wave propagation (PWP) model. Additionally, we showcase the integrated CircAdapt-TL model, which now includes the heart as well as extensive arterial and venous trees with terminal impedances. We present CircAdapt-TL haemodynamics simulations of 1) a systemic normotensive situation and 2) a systemic hypertensive situation.

In the TL-PWP benchmark comparison we found good agreement regarding pressure and flow waveforms (relative errors $\leq 2.9 \%$ for pressure, and $\leq 5.6 \%$ for flow). CircAdapt-TL simulations reproduced the typically observed haemodynamic changes with hypertension, expressed by increases in mean and pulsatile blood pressures, and increased arterial pulse wave velocity. We observed a change in the timing of pressure augmentation (defined as a late-systolic boost in aortic pressure) from occurring after time of peak systolic pressure in the normotensive situation, to occurring prior to time of peak pressure in the hypertensive situation. The pressure augmentation could not be observed when the systemic circulation was lumped into a (non-linear) three-element windkessel model, instead of using our TL model. Wave intensity analysis at the proximal aorta indicated earlier arrival and increased intensity of reflected waves with hypertension as compared to normotension, in good qualitative agreement with findings in patients.

In conclusion, we successfully embedded a TL model as a vascular module into the CircAdapt platform. The integrated CircAdapt-TL model allows mechanistic studies on heart-vessel interaction. 


\subsection{Introduction}

HE CircAdapt platform, a zero-dimensional whole-heart model developed in our lab, historically focussed on cardiac mechanics. It has been successfully used for simulating haemodynamics during cardiac conductance disorders, valve pathologies, and changes in afterload [23, 26, 34, 43]. Lacking a distributed model of the vascular system, the current CircAdapt model is yet unable to simulate heart-vessel interaction at the level of arterial wave dynamics.

Arterial pulse waves, constituting a component of ventricular afterload, appear to have implications in age-related changes in left ventricular mass, and left ventricular hypertrophy $[12,29]$. So-called wave intensity analysis (WIA) allows characterisation of both pulse wave magnitude and propagation direction, thereby requiring synchronous and co-localised measurements of blood pressure and blood flow velocity signals [35]. WIA applied to patient measurement data is sensitive to synchronisation errors and the signal processing characteristics of the measurement devices [37], which hampers or limits detailed studies on heart-vessel interaction, especially concerning causal relationships.

Computational models of whole-circulation mechanics, such as CircAdapt, allow for well-controlled simulations, facilitating comprehensive study of singleand multi-factorial relationships between arterial system properties and cardiac structure and function. In the present study we introduce and demonstrate the CircAdapt-TL model (Fig. 3.1): a whole-circulation model with an integrated segmental transmission line (TL) module, describing vascular wave propagation, reflection and transmission. We verify the numerical implementation of the TL model by a benchmark comparison of the model to the established pulse wave propagation (PWP) model of Kroon et al. [21]. Additionally, we demonstrate operation and output of the integrated CircAdapt-TL model, by simulating systemic normotensive- and hypertensive conditions. We evaluate the implications of modelling vascular wave transmission on aortic haemodynamics by comparing simulated left ventricular- and aortic pressure tracings of the integrated CircAdapt-TL model with the tracings obtained with the systemic circulation lumped into the existing CircAdapt non-linear three-element windkessel (3WK) model (i.e. neglecting wave transmission effects). Further evaluation includes WIA applied to simulated carotid arterial pressure and flow waveforms in semi-quantitative comparison with WIA applied to patient measurements.

\subsection{Models}

\subsubsection{Overview of complete model}

Our vascular module describing wave transmission in vascular networks will be integrated into the existing CircAdapt platform (www . circadapt . org). This model platform has a modular setup, currently consisting of a 0D whole-heart mechanics model, valve haemodynamics model, and non-linear three-element 


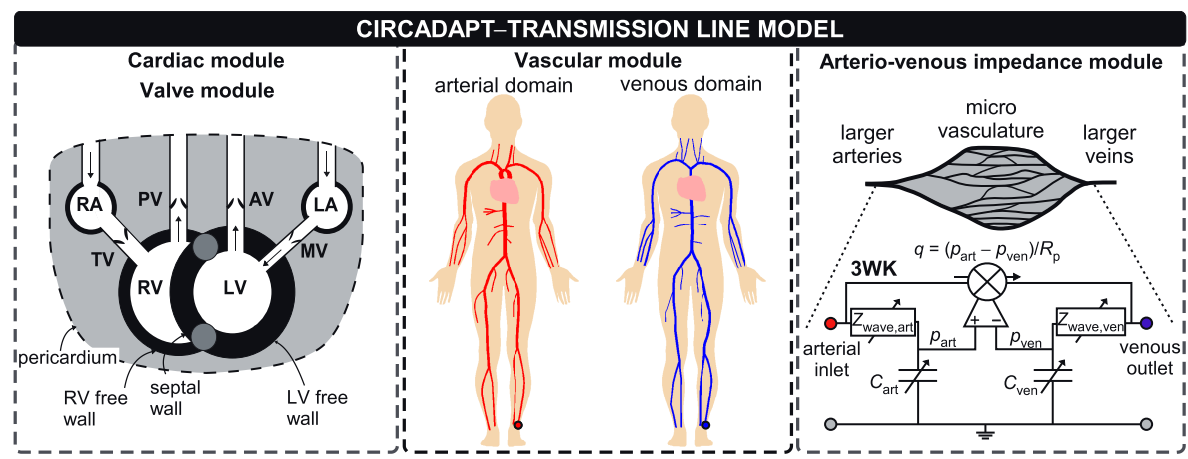

Figure 3.1: The new vascular module interfaces the existing whole heart-heart mechanics and arterio-venous impedance modules. The CircAdapt-TL model contains the cardiac modules describing whole-heart mechanics, including interventricular interactions, and the systemic and pulmonary circulations [25]. Cardiac valves are modelled as described in Palau-Caballero et al. [34]. The vascular module is described in section 'Vascular module'. The arterio-venous impedance module, modelling the peripheral circulation using a non-linear three-element windkessel (3WK) was previously developed by Arts et al. [3]. Abbreviations: RA: right atrium, LA: left atrium, TV: tricuspid valve, PV: pulmonary valve, AV: aortic valve, RV: right ventricle, LV: left ventricle, MV: mitral valve, art: arterial, ven: venous, $3 \mathrm{WK}$ : non-linear three-element windkessel model. Symbols: $R_{\mathrm{p}}$ : peripheral resistance, $Z_{\text {wave }}$ : wave impedance, $C$ : compliance.

windkessel models of the pulmonary and peripheral circulations (Fig. 3.1). In the next section, we introduce the governing equations, modelling assumptions and implementation of our new vascular module in detail.

\subsubsection{New vascular TL-module}

To model pressure-flow waves within segments of blood vessels, we assume 1) blood vessels to be thick-walled, longitudinally constrained non-linear elastic tubes, 2) blood to be incompressible and Newtonian and 3) that gravity forces can be neglected. Furthermore, we assume 4) no leakage of blood to small sidebranches that are not explicitly modelled. Application of the laws of balance of mass and momentum, and subsequent integration over the tube's cross-sectional area yield the governing equations [16]:

$$
\begin{aligned}
& C \frac{\partial p}{\partial t}+\frac{\partial q}{\partial z}=0 \\
& L\left(\frac{\partial q}{\partial t}+\frac{\partial}{\partial z} \int_{A} v_{z}^{2} d A\right)+\frac{\partial p}{\partial z}=f
\end{aligned}
$$

where $p=p(z, t)$ is the pressure at the axial vessel coordinate $z$, and $q=q(z, t)$ the flow rate at that coordinate. Furthermore, $A$ denotes cross-sectional lumen area, 
and $L$ and $C$ are the tube inertance and compliance per unit length, respectively. Term $L \frac{\partial}{\partial z} \int_{A} v_{z}^{2} d A$ represents the convective acceleration term, with $v_{z}$ the axial blood velocity. Term $f$ represents friction force per unit volume caused by viscous properties of the blood, defined $f=2 \pi r_{0} \tau_{\mathrm{w}} / A_{0}$ [6]. Here, symbol $\tau_{\mathrm{w}}$ denotes wall shear stress, $r_{0}$ reference radius, and $A_{0}$ reference cross-sectional lumen area, respectively. After neglecting the convective acceleration term and assuming an approximate velocity profile to estimate $\tau_{\mathrm{w}}[6]$, the governing equations can be rewritten to the telegrapher's equations:

$$
\begin{aligned}
& -\frac{\partial q}{\partial z}=C \frac{\partial p}{\partial t} \\
& -\frac{\partial p}{\partial z}=L\left(\alpha_{0}\right) \frac{\partial q}{\partial t}+R\left(\alpha_{0}\right) q
\end{aligned}
$$

with $L\left(\alpha_{0}\right)$ and $R\left(\alpha_{0}\right)$ a characteristic Womersley number-dependent inertance and resistance term, defined by

$$
\begin{aligned}
L\left(\alpha_{0}\right) & =g\left(\alpha_{0}\right) \frac{\rho}{A_{0}}, \\
R\left(\alpha_{0}\right) & =h\left(\alpha_{0}\right) \frac{8 \pi \eta}{A_{0}^{2}}, \text { with } \\
\alpha_{0} & =r_{0} \sqrt{\rho \omega_{0} / \eta} .
\end{aligned}
$$

The functions $g\left(\alpha_{0}\right)$ and $h\left(\alpha_{0}\right)$ were derived by Bessems et al. [6] and are detailed in Appendix 3.A1.2. The characteristic Womersley number $\left(\alpha_{0}\right)$ describes the ratio of instationary inertia forces and viscous forces, governed by vessel radius $\left(r_{0}\right)$, characteristic angular frequency $\left(\omega_{0}=2 \pi / T\right.$, with $T$ the cardiac cycle duration), blood dynamic viscosity $(\eta)$ and blood density $(\rho)$, respectively (Table 3.1).

Table 3.1: Parameters relevant for the transmission line model.

\begin{tabular}{cccll}
\hline Symbol & Value or expression & Unit & Meaning & Reference \\
\hline$A_{0}, r_{0}$ & See Table A2 and A3 & $\mathrm{m}^{2}$ or m & Reference lumen area or vessel radius & {$[30,39]$} \\
$\alpha_{0}$ & $\alpha_{0}=r_{0} \sqrt{\rho \omega_{0} / \eta}$ & - & Characteristic Womersley number & {$[6]$} \\
$b$ & 0.02 & - & Collapsible tube fraction & Assumed \\
$\Delta t$ & 0.001 & $\mathrm{~s}$ & Time step & Assumed \\
$\Delta z$ & 0.02 & $\mathrm{~m}$ & Element size & Assumed \\
$l_{\mathrm{AV}}$ & $6 q_{\mathrm{AV}}^{1 / 3}$ & $\mathrm{~m}$ & Characteristic vessel bed length & Assumed \\
$k$ & See Tables A2 and A3 & - & Vessel stiffness coefficient & {$[13]$, Assumed } \\
$l$ & See Tables A2 and A3 & $\mathrm{m}$ & Vessel length & {$[30,39]$} \\
$\eta$ & 0.003 & $\mathrm{~Pa} \mathrm{~s}$ & Blood dynamic viscosity & {$[47]$} \\
$p_{0, \mathrm{a}}$ & 105 (REF), $135(\mathrm{HYP})$ & $\mathrm{mmHg}$ & Reference pressure for arteries & Assumed \\
$p_{0, \mathrm{v}}$ & 1.1 (REF), 1.1 (HYP) & $\mathrm{mmHg}^{-1}$ & Reference pressure for veins & Assumed \\
$q_{\mathrm{AV}}$ & See Table A2 & $\mathrm{ml} \mathrm{s}^{-1}$ & Mean flow through terminal tube & {$[11,14]$, Assumed } \\
$q_{0}$ & 85 & $\mathrm{ml} \mathrm{s}^{-1}$ & Mean systemic flow & {$[43]$} \\
$\rho$ & 1050 & $\mathrm{~kg} \mathrm{~m}^{-3}$ & Blood density & {$[47]$} \\
$T$ & 0.85 & $\mathrm{~s}$ & Cardiac cycle duration & {$[43]$} \\
$\omega_{0}$ & 7.39 & rad s & Characteristic angular frequency & {$[6]$} \\
\hline
\end{tabular}

REF: Reference simulation. HYP: Hypertension simulation. 
The rationale of this method is to calculate $R$ and $L$ based on an approximated velocity profile for which the viscous boundary layer thickness is approximated for the characteristic frequency [6]. To solve the governing equations, we also need a constitutive law to relate (changes in) transmural pressure ( $\left.p_{\text {trans }}\right)$ to (changes in) current cross-sectional area $(A)$. We formulated a non-linear power-law to phenomenologically capture the experimentally observed non-linear pressurearea relation of arteries and veins $[4,13]$ :

$$
\begin{aligned}
p_{\text {trans }}(A) & =-p_{\text {ext }}+p_{0}\left((1+b)\left(\frac{A}{A_{0}}\right)^{1+\frac{k / 3-2}{1+b}}-\frac{b A_{0}}{A}\right), \\
& \text { and } \\
C & =\frac{\mathrm{d} A}{\mathrm{~d} p_{\text {trans }}},
\end{aligned}
$$

with $p_{0}$ a reference pressure, $A_{0}$ a reference cross-sectional area, and $k$ the vessel stiffness coefficient. Furthermore, $b$ is a small fraction to simulate collapse of the tube with negative transmural pressure (Table 3.1) and $p_{\text {ext }}$ represents a prescribed external pressure (if present). Now we can solve the resulting governing equations in either the time domain or frequency domain [10]. We explicitly chose a time-domain approach, since this permits using non-linear boundary conditions as already present in the CircAdapt platform [43]. Our solving method uses a TL model. A detailed overview of our solving method is provided in Appendix 3.A1.1.

\subsubsection{Arterio-venous impedance module}

The terminal end of a tube was coupled to a non-linear three-element windkessel (3WK) element [3]. We assumed the windkessel compliance to be pressuredependent, and scaled by an estimate of the tissues' vessel bed length [46]. As a consequence, wave impedance also becomes pressure-dependent (Fig. 3.1):

$$
C_{\mathrm{AV}}=l_{\mathrm{AV}} \frac{\mathrm{d} A}{\mathrm{~d} p_{\mathrm{AV}}} \text { and } Z_{\mathrm{wave}, \mathrm{AV}}=\sqrt{\frac{\rho}{A} \frac{\mathrm{d} p_{\mathrm{AV}}}{\mathrm{d} A}},
$$

with $\mathrm{AV}$, the subscript for the arterial and venous contributions, i.e. $\mathrm{AV}=$ [art,ven]. Such approach enables simulating large changes in haemodynamic load (e.g. exercise or hypertension) without requiring to manually adjust the $3 \mathrm{WK}$ parameter values. The derivatives in the aforementioned equations were calculated at the connection point (i.e. a node) of a tube with a $3 \mathrm{WK}$, using the constitutive relation as given in Eq. 4.2. The parameter $l_{\mathrm{AV}}$ represents the characteristic length of a peripheral bed. We estimated the vessel bed length using the relation given by $l_{\mathrm{AV}}=6 q_{\mathrm{AV}}^{1 / 3}$, with $q_{\mathrm{AV}}$ the mean peripheral flow through any terminal tube. To obtain first-order approximations of $l_{\mathrm{AV}}$ among all peripheral beds, we utilised this relation in conjunction with flow distribution estimates as reported in Table A2. Furthermore, using a physiology textbook [11], we estimated that in rest 
$21 \%$ of the cardiac output is directed to the head, $47 \%$ to the abdomen, $18 \%$ to the pelvis and lower extremities, and $14 \%$ to the upper extremities, respectively. The peripheral resistance $\left(R_{\mathrm{p}}\right)$ was defined via a flow source, controlled by the instantaneous arterio-venous pressure difference (Fig. 3.1) [3]:

$$
R_{\mathrm{p}}=\frac{p_{\mathrm{art}}-p_{\mathrm{ven}}}{q_{\mathrm{AV}}} .
$$

\subsubsection{Cardiac module}

The atria and ventricles of the heart were modelled as contractile chambers. The ventricles are surrounded by three cardiac walls: the left ventricle free wall, interventricular septum and right ventricle free wall (Fig. 3.1). Ventricles are mechanically coupled, based on force equilibrium in the junction of the ventricular walls [25]. The atria are surrounded by the left atrial wall and right atrial wall (Fig. 3.1). The cardiac chambers are considered as contractile cavities formed by the one-fibre model, relating myofibre stress to cavity pressure using the assumption that myofibre stress is homogeneously distributed within the myocardial wall [1]. The phenomenological model of myofibre mechanics was previously described [43]. The one-fibre model is used to calculate myofibre stress from myofibre strain. Total Cauchy myofibre stress experienced by cardiac tissue comprises of a summation of active stress, present in the actin filaments and separate microstructural contributions (i.e. titin and the extracellular matrix, assumed to act in parallel). Transmural pressure is calculated from wall tension, derived from total Cauchy stress and wall curvature using Laplace's law [43]. Cavity pressures are calculated by adding the transmural pressures to the pericardial pressure surrounding the myocardial walls. As commonly used in other cardiac models, the pericardium was assumed a compliant bag, modelled using a non-linear relation relating pericardial pressure and volume [19]. The pulmonary circulation was modelled as 3WK (see Section 'Arterio-venous impedance module'), connecting the pulmonary artery with the pulmonary veins [33]. Full details of the cardiac model can be retrieved from Walmsley et al. [43] and Lumens et al. [25].

\subsubsection{Valve module}

Valve flow $\left(q_{\text {valve }}\right)$ was generated using the unsteady Bernoulli equation, assuming incompressibility and inviscid, irrotational flow:

$$
\frac{\rho l_{\text {valve }}}{A_{\text {valve }}} \frac{\partial q_{\text {valve }}}{\partial t}=\Delta p-\frac{1}{2} \rho q_{\text {valve }}\left|q_{\text {valve }}\right|\left(\frac{1}{A_{\text {valve }}^{2}}-\frac{1}{A_{\mathrm{p}}^{2}}\right),
$$

with the term on the left hand side the unsteady inertia, governed by blood density, effective valve length $\left(l_{\text {valve }}\right)$ and valve cross-sectional area $\left(A_{\text {valve }}\right)$ [34]. The first term on the right hand side denotes the pressure difference $(\Delta p)$ and the second term is the change in kinetic energy, with $A_{\text {valve }}$ and $A_{\mathrm{p}}$ cross-sectional areas of 
the valve and proximal to the valve, respectively. For $A_{\text {valve }}$, a phenomenological valve opening and closing function depending on the pressure gradient was used [34]. In case $\Delta p>0, A_{\text {valve }}$ instantaneously increases towards an effective valve area representing a completely opened valve. In case $\Delta p<0$, on the other hand, flow gradually decreases due to inertia. Furthermore, $A_{\text {valve }}$ gradually decreases towards a quasi-closed state, with small leakage to avoid division by zero [34].

\subsubsection{Simulations and analysis}

\section{Benchmark comparison}

First, we evaluated the TL model's numerical framework in a benchmark comparison, comparing arterial pressure and flow waveforms generated by on the one hand the TL model and those generated by the PWP model, developed by Kroon et al. [21]. The numerical framework of this PWP model was previously validated in Boileau et al. [8] against in vitro experiments, whereas Bessems et al. [6] confirmed the validity of the approximate velocity profile against Womersley theory. In the benchmark comparison, we considered large central arteries (vessel diameters between 15 and $30 \mathrm{~mm}$ ) as well as smaller arteries of the left arm (vessel diameters between 2 and $11 \mathrm{~mm}$, Table A2). Furthermore, the same set of equations (i.e. the balance equations and the constitutive law) was solved for the PWP- as well as for the TL model. We also kept boundary conditions (i.e. constitutive law and 3WK parameters) equal between the PWP- and TL model. At the proximal aorta we prescribed a periodical flow wave $\left(q_{\text {inflow }}\right)$ with a period of $0.85 \mathrm{~s}$. This flow wave was composed of a half-sinusoidal wave with a duration $\left(t_{\mathrm{c}}\right)$ of $0.3 \mathrm{~s}$ and a peak flow rate $\left(q_{\mathrm{p}}\right)$ of $350 \mathrm{ml} \mathrm{s}^{-1}$, whereas flow was zero in the rest of the period,

$$
q_{\text {inflow }}(t)= \begin{cases}q_{\mathrm{p}} \sin \left(\frac{\pi t}{t_{\mathrm{c}}}\right) & \text { if } 0 \leq t \leq t_{\mathrm{c}} \\ 0 & \text { if } t>t_{\mathrm{c}} .\end{cases}
$$

The PWP model used a simplified trapezoidal scheme for spatial discretisation and a second-order backward difference scheme for time discretisation. For each tube in the domain, agreement between models in terms of pressure and flow, was quantified by calculation of root mean square errors $(\epsilon)$ and relative errors 
$(\delta):$

$$
\begin{aligned}
& \epsilon_{p, n}=\sqrt{\frac{\sum_{t=0}^{T}\left(p_{\mathrm{TL}}(t)-p_{\mathrm{PWP}}(t)\right)^{2}}{N_{\mathrm{t}}}}, \\
& \epsilon_{q, n}=\sqrt{\frac{\sum_{t=0}^{T}\left(q_{\mathrm{TL}}(t)-q_{\mathrm{PWP}}(t)\right)^{2}}{N_{\mathrm{t}}}}, \\
& \delta_{p, n}=\frac{1}{N_{t}} \sum_{t=0}^{T}\left|\frac{p_{\mathrm{TL}}(t)-p_{\mathrm{PWP}}(t)}{p_{\mathrm{PWP}}(t)}\right| \cdot 100 \%, \text { and } \\
& \delta_{q, n}=\frac{1}{N_{t}} \sum_{t=0}^{T}\left|\frac{q_{\mathrm{TL}}(t)-q_{\mathrm{PWP}}(t)}{\max _{n}\left(q_{\mathrm{PWP}}(t)\right)}\right| \cdot 100 \%,
\end{aligned}
$$

with $n$ the tube reference number, $T$ the cardiac cycle duration and $N_{t}$ the number of time points for which a comparison is made. A complete overview of the benchmark comparison setup is provided in Appendix 3.A2.

\section{Simulation of arterial and venous haemodynamics using the CircAdapt-TL model}

We first show the applicability of the CircAdapt-TL model in terms of simulation of pressure and flow waveforms throughout arteries and veins as well as changes in wave dynamics when simulating systemic hypertension. Furthermore, we compare LV and aortic pressure tracings of the CircAdapt-TL model with those obtained when lumping the systemic arterial and venous blood vessels into a single 3WK. For the first purpose, we implemented an arterial tree (Fig. 3.2 and Table A2), for which the morphological and mechanical properties were based on the work of Reymond et al. [39], and a venous tree (Fig. 3.3 and Table A3), based on the work of Müller and Toro [30]. Since in the present study we were not interested in simulating pressure and flow in cerebral and coronary vessels, we excluded these vessels from our model domain. Vessel stiffness coefficients $(k)$ for the aortic segments as well as arteries of the left and right arm were as used in the benchmark comparison. Furthermore, for the arteries of the leg (i.e. the iliac, femoral and tibial arteries), we chose $k$ equal to 30, based on data of Hayashi et al. [13]. Given the lack of human data on veins, a fixed $k$-value of 10 was chosen for all veins in the model domain.

We performed the following sets of simulations.

1) Using the CircAdapt-TL model, we performed:

- A reference simulation (REF-TL) in which we model the case of normal arterial stiffness. For this simulation, we kept reference pressure $\left(p_{0}\right)$ at $105 \mathrm{mmHg}$ and vessel stiffness coefficient $(k)$ as given in Tables A2 and A3. According to clinical standards, we characterised arterial stiffness by calculating the carotid-femoral pulse wave velocity (PWV). We obtained 
PWV by dividing the fixed path length between the terminal nodes of the tubes that modelled the carotid artery and femoral artery by the pulse transit time between these nodes. The pulse transit time was obtained as the footto-foot time difference between the respective pressure waveforms. Foot detection was performed using the maximum of the $2^{\text {nd }}$-order derivative of the pressure waveform.

- A systemic hypertension simulation (HYP-TL), in which we model a hypertensive situation. For this simulation, we increased $p_{0}$. Such increase in $p_{0}$ can physiologically be interpreted as an increase in peripheral resistance. Furthermore, vascular stiffness parameter $k$ of all systemic arterial segments was increased by a factor $\Delta k$. The latter increases the nonlinearity of the pressure-area relation (Eq. 4.2), and models an increase in material stiffness of the vessel wall. For the HYP simulation, we imposed $\left[p_{0}, \Delta k\right]=[135 \mathrm{mmHg},+6]$. This resulted in a blood pressure exceeding 140/90 mmHg, a situation defined as hypertension according to the European Society of Hypertension/European Society of Cardiology guidelines [49].

2) Using the CircAdapt-3WK model, we performed

- A reference and hypertension simulation. Now, the systemic circulation was lumped into a 3WK (REF-3WK and HYP-3WK, see Appendix 3.A3).

For the REF-TL and HYP-TL simulations, we compared pressure and flow waveforms for cardiac chambers, central arteries and veins, as well as trans-valvular flows. Furthermore, haemodynamic indices (i.e. diastolic, systolic blood pressures, pulse wave velocity) were compared between the REF-TL and HYP-TL simulation. Systolic and diastolic aortic blood pressures were calculated from the tube that mimics the ascending aorta (i.e. tube \#1, Fig. 3.2). The following analyses were conducted to assess the implications of using the TL model on aortic haemodynamics. First, we compared the morphology of pressure tracings of the LV and aorta obtained using the CircAdapt-TL model with those from the CircAdapt-3WK model. Second, wave intensity analysis was performed in the common carotid artery. Wave intensity analysis provides an approach to determine the characteristics of the primary wave originating from wave reflection, (i.e. termed the backward compression wave (BCW)) [35]. Wave intensity may be defined as the rate of energy transfer per unit area, often given in units of $\left[\mathrm{W} \mathrm{m}^{-2} \mathrm{~s}^{-2}\right]$ [48]. Wave intensity is positive $\left(\mathrm{d} I^{+}\right)$for a forward running wave, and negative $\left(\mathrm{d} I^{-}\right)$for a backward running wave. Furthermore, net wave intensity $(\mathrm{d} I)$ is defined as the sum of backward and forward wave intensity, respectively. Using the derived wave intensity tracings, we qualitatively assessed changes in arrival time and intensity of the BCW between the normotensive- and hypertensive situations. Finally, we compared between simulated wave intensity tracings and those obtained in patients. Details regarding derivation of $\mathrm{d} I^{+}, \mathrm{d} I^{-}$, and $\mathrm{d} I$ are provided in Appendix 3.A5. 


\section{Numerical implementation}

All simulations were performed in MATLAB 2015a (The MathWorks, Natick, MA, USA) on a standard personal computer running an Intel ${ }^{\circledR}$ Core $i 7^{\text {TM }}$ processor and 8.00 GB RAM. Blood viscosity was kept at $3 \cdot 10^{-3}$ Pa s, blood density was kept at $1050 \mathrm{~kg} \mathrm{~m}^{-3}$, and collapsible tube fraction $b$ was kept at 0.02 (Table 3.1 ). We chose a time step $(\Delta t)$ of $1 \mathrm{~ms}$. Furthermore, for the simulations performed using the CircAdapt-TL model, we chose an element size (i.e. $\Delta z$, indicating the distance between nodes) of $0.02 \mathrm{~m}$. This value was chosen as a trade-off between on the one hand being able to capture geometric tapering of arterial vessels, and on the other hand to restrain simulation time. For both the benchmark evaluation and the simulations that were performed using the CircAdapt-TL and CircAdapt-3WK models, we ensured that numerical convergence was achieved (i.e. negligible change in pressure and flow waveforms when further decreasing $\Delta t$ and $\Delta z$ ).

\subsection{Results}

\subsubsection{Benchmark comparison}

Agreement between pressure and flow waves of the TL model and PWP model for five tubes in the model domain is graphically depicted in Fig. 3.4. Root mean square errors (Eq. 3.13) for pressures and flows for all tubes are given in Table 3.2 .

Table 3.2: Benchmark comparison between pressure and flow waveforms of the new transmission line (TL) model and established pulse wave propagation (PWP) model.

\begin{tabular}{lccccccccccccc}
\hline tube \# & $1+2$ & 4 & 15 & $19+27$ & 3 & 16 & 20 & 21 & 22 & 23 & 24 & 26 & 25 \\
\hline$\epsilon_{p}[\mathrm{mmHg}]$ & 1.62 & 1.65 & 1.68 & 1.62 & 2.36 & 1.68 & 1.68 & 2.14 & 2.96 & 3.96 & 3.68 & 4.03 & 4.05 \\
$\epsilon_{q}\left[\mathrm{ml} \mathrm{s}^{-1}\right]$ & 2.53 & 21.20 & 4.41 & 5.71 & 3.02 & 0.87 & 1.63 & 0.32 & 0.92 & 0.22 & 0.48 & 0.30 & 0.27 \\
$\delta_{p}[\%]$ & 0.8 & 0.8 & 0.8 & 0.9 & 1.2 & 0.8 & 0.8 & 1.5 & 1.5 & 2.7 & 2.2 & 2.8 & 2.9 \\
$\delta_{q}[\%]$ & 0.6 & 5.6 & 1.1 & 1.9 & 4.5 & 2.2 & 3.3 & 2.8 & 3.5 & 3.2 & 3.1 & 3.7 & 5.3 \\
\hline
\end{tabular}

Root mean square errors for pressure $\left(\epsilon_{p}\right)$ and for flow $\left(\epsilon_{q}\right)$, as well as their relative errors $\left(\delta_{p}\right.$ and $\left.\delta_{q}\right)$ describe agreement between both models. Agreement was calculated for the element located at the centre of the tube.

Between models, we found good agreement in terms of pressure and flow waveforms for proximal arteries (e.g. aorta, carotid, subclavian and vertebral arteries), expressed by relative errors $\delta_{p} \leq 1.5 \%$ and $\delta_{q} \leq 5.6 \%$ At the distally located interosseous artery, the difference between both models slightly increased, expressed by $\delta_{p, 25}$ equal to $2.9 \%$ and $\delta_{q, 25}$ equal to $5.3 \%$. Nevertheless, the shape of the pressure and flow waveforms as well as absolute systolic and diastolic pressure and flow values were highly similar (Fig. 3.4). 


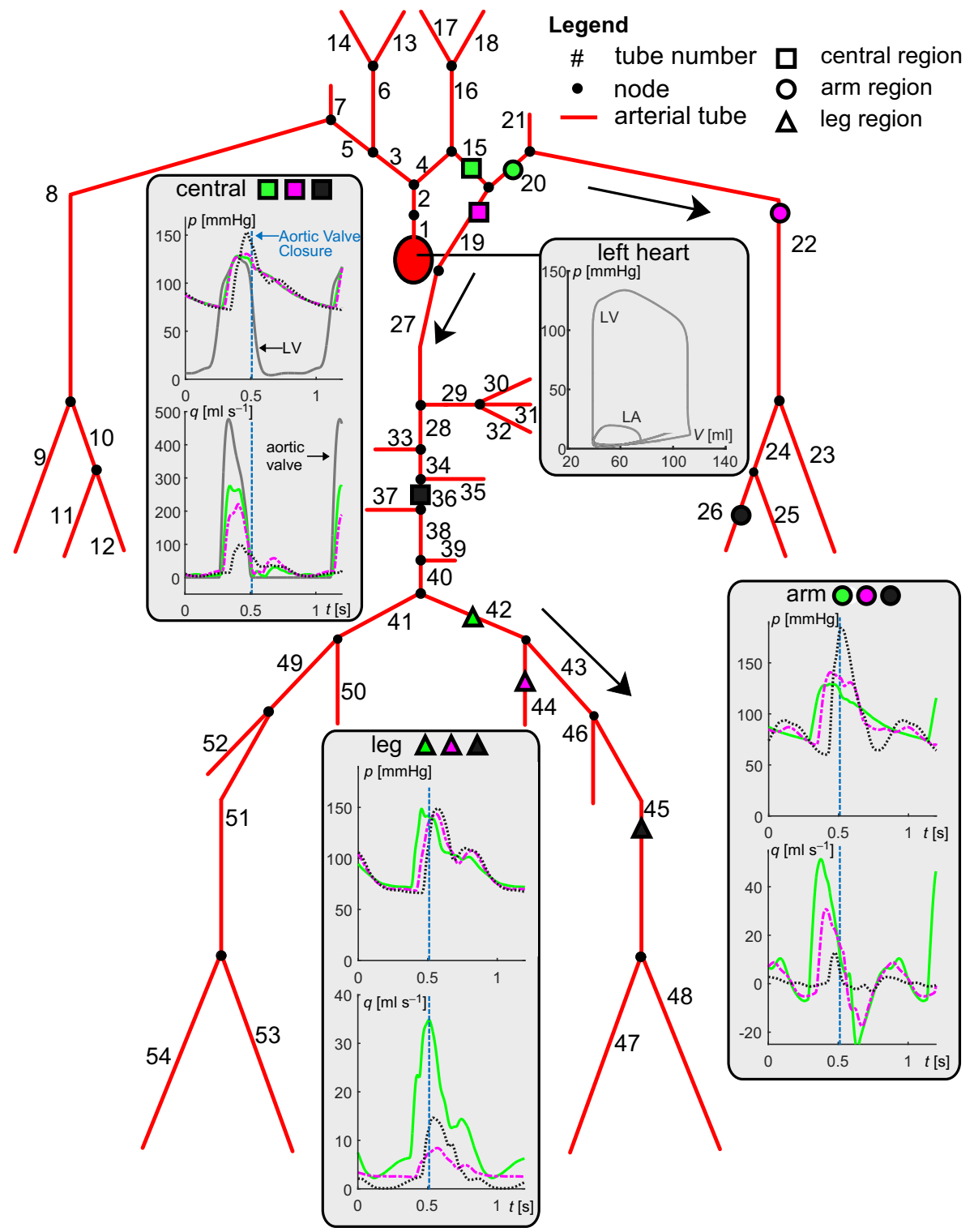

Figure 3.2: Overview of the complete arterial model domain. Geometrical and mechanical properties of modelled arteries are given in Table A2. Panels display pressure and flow waveforms for three regions (i.e. central, arm and leg, indicated using symbol and colour coding). Grey curves display left ventricular (LV) pressures and volumes, left atrial (LA) pressures and volumes as well as aortic valve flow. Line type indicates the distance from the heart within a region; proximal: solid line, intermediate: dash-dotted line, distal: dotted line, respectively. Arrows indicate the direction of mean blood flow. Aortic valve closure is indicated by the vertical blue dashed lines. 


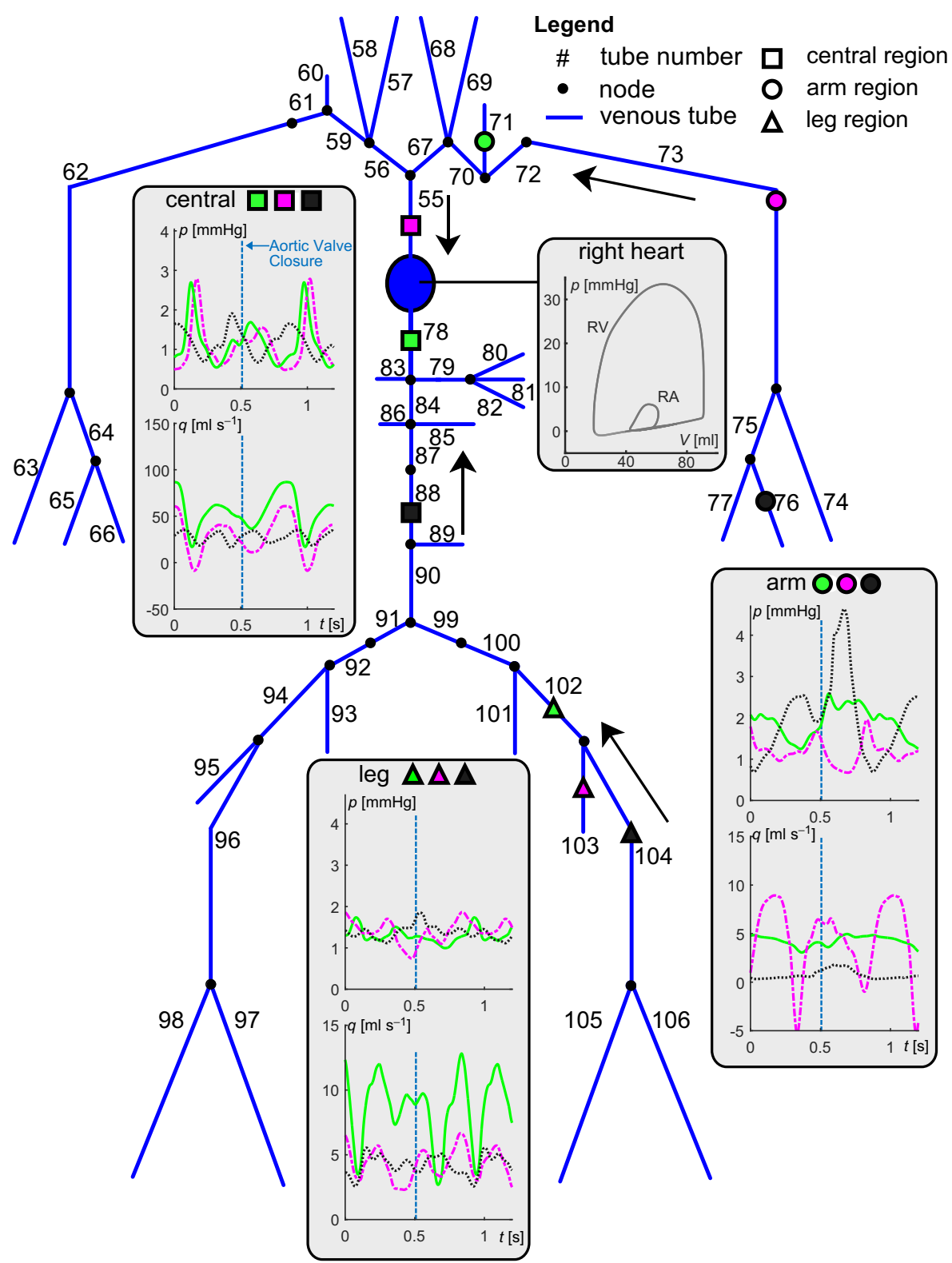

Figure 3.3: Overview of the complete venous model domain. Geometrical and mechanical properties of modelled veins are given in Table A3. Panels display pressure and flow waveforms for three regions; central, arm and leg, indicated using symbol and colour coding. Line type indicates the distance from the heart within a region; proximal: solid line, intermediate: dash-dotted line, distal: dotted line, respectively. Grey curves display right ventricular (RV) pressures and volumes, right atrial (RA) pressures and volumes. Arrows indicate the direction of mean blood flow. Aortic valve closure is indicated by the vertical blue dashed lines. 




Figure 3.4: Results of the benchmark comparison. Pressure $(p)$ and flow $(q)$ waveforms generated by the transmission line (TL) model and 1D pulse wave propagation model (PWP) model are shown for various locations along the arterial domain. Agreement between $p$ and $q$ waveforms is expressed by root mean square errors $\epsilon_{p}$ and $\epsilon_{q}$, as well as relative errors $\delta_{p}$ and $\delta_{q}$.

\subsubsection{Simulation of normotension and hypertension}

In Figs 3.2 and 3.3, pressure and flow waveforms in normotension are displayed for arteries and veins at three regions (i.e. the central-, arm- and leg region). Arterial pressure waveforms at distal locations are characterised by an increase in pressure amplitude, as well as a reduction in peak width. The arterial pressure waveforms at distal locations show a more prominent dicrotic notch compared to the pressure waves at proximal locations. For veins, a biphasic pressure waveform can be distinguished, with venous flow and pressure in anti-phase (Fig. 3.3).

In the REF-TL simulation, pulse wave velocity (PWV) was $5.5 \mathrm{~m} \mathrm{~s}^{-1}$, representing a PWV value commonly found in subjects aged $<30$ years [28]. For the HYP simulation, pulse wave velocity (PWV) was $8.0 \mathrm{~m} \mathrm{~s}^{-1}$, representing a PWV value clinically associated with early aortic stiffening, and commonly found in subjects aged $>50$ years [28]. The blood pressure values in the REF-TL simulation were within the normal range (Table 3.3). As shown in Fig. 3.5, simulating systemic hypertension (HYP) caused arterial pressure to increase. This resulted in an increase in left ventricular pressure and left atrial pressure, whereas pulmonary 
artery pressure and pulmonary venous pressure slightly increased (Fig. 3.5). The HYP-TL simulation showed an increase in systolic blood pressure $\left(p_{\text {sys }}\right)$ from 128 to $193 \mathrm{mmHg}$ and an increased diastolic blood pressure ( $\left.p_{\text {dia }}\right)$ from 75 to $92 \mathrm{mmHg}$ (Table 3.3).
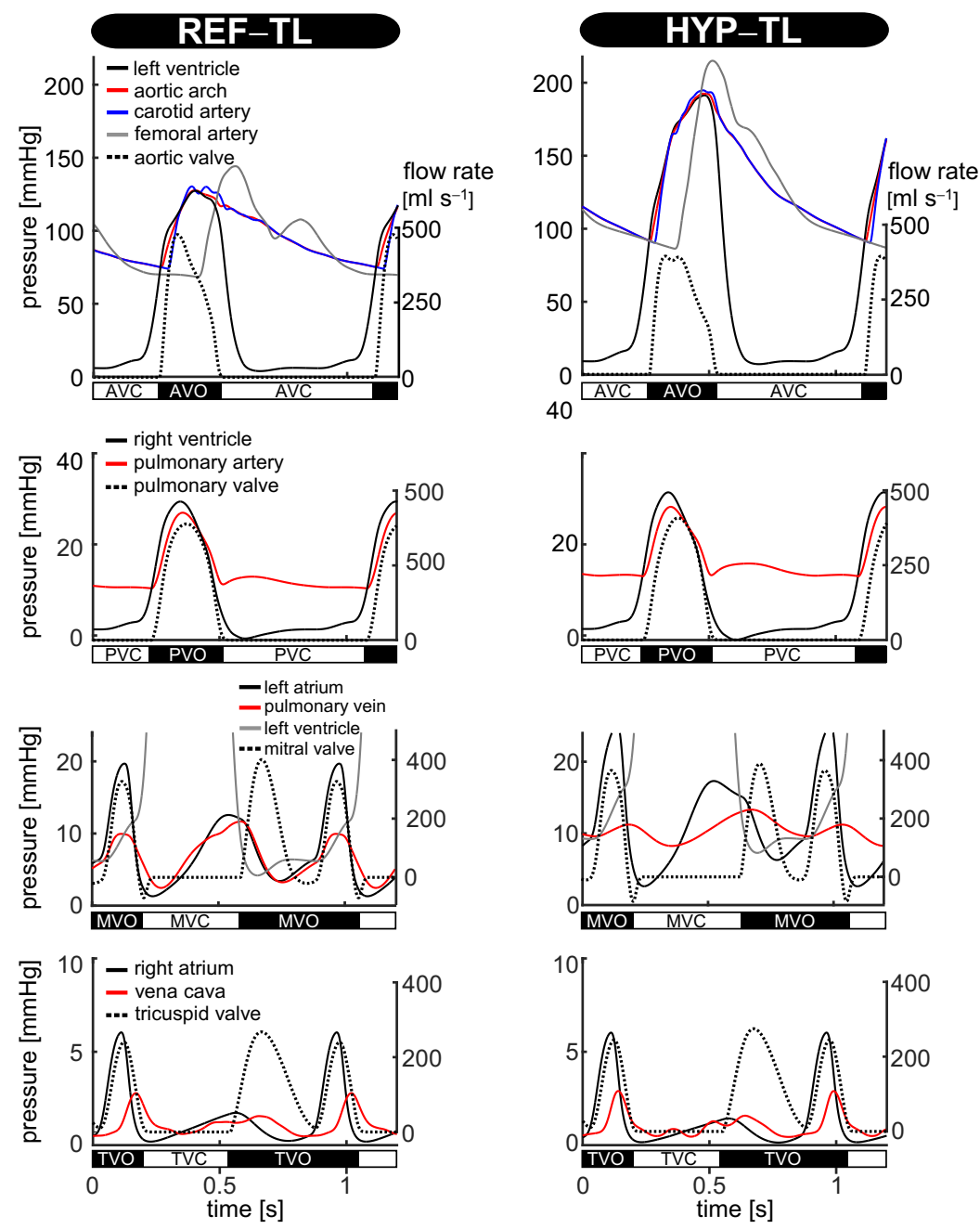

Figure 3.5: CircAdapt-TL model simulated time courses of pressure and flow for the reference (REF-TL) simulation (left), and the hypertension (HYP-TL) simulation (right). Curves display time courses of pressure (solid lines) and flow (dotted lines) of the left ventricle and large arteries; the right ventricle and pulmonary artery; the left atrium and pulmonary vein; and the right atrium and vena cava, respectively. AVC: aortic valve closed, AVO: aortic valve open, MVC: mitral valve closed, MVO: mitral valve open, PVC: pulmonary valve closed, PVO: pulmonary valve open, TVC: tricuspid valve closed, TVO: tricuspid valve open. 
Table 3.3: Haemodynamic indices in the reference (REF) and hypertensive (HYP) simulations.

\begin{tabular}{ccccc}
\hline Index & REF & HYP & Unit & Meaning \\
\hline$p_{\text {sys }}$ & 128 & 193 & $\mathrm{mmHg}$ & Systolic pressure in the aorta \\
$p_{\text {dia }}$ & 75 & 92 & $\mathrm{mmHg}$ & Diastolic pressure in the aorta \\
$p_{\text {pulse }}$ & 53 & 101 & $\mathrm{mmHg}$ & Pulse pressure in the aorta \\
PWV & 5.5 & 8.0 & $\mathrm{~m} \mathrm{~s}^{-1}$ & Carotid-femoral pulse wave velocity \\
\hline
\end{tabular}

Fig. 3.6 shows LV and ascending aortic pressure tracings obtained using the CircAdapt-3WK model and the CircAdapt-TL model, respectively. The aortic pressure tracings of the REF-TL and HYP-TL simulation showed pressure augmentation (i.e. a systolic pressure boost) as well as an dicrotic notch, whereas for CircAdapt-3WK model simulations, these waveform characteristics were absent. In the REF-TL simulation, systolic pressure augmentation occurred after time of peak systolic pressure, whereas in the HYP-TL simulation this occurred prior to the time of peak systolic pressure.

Wave intensity tracings $\left(\mathrm{d} I^{+}, \mathrm{d} I^{-}\right.$, and $\mathrm{d} I$, respectively) of the REF-TL and HYP-TL simulation were calculated for the left common carotid artery (Fig. 3.7A). The carotid arterial wave intensity tracings of the REF-TL simulation indicate a forward compression wave (FCW) followed by a backward compression wave (BCW). At end-systole, there is a forward expansion wave (FEW) associated with the deceleration of the rate of myocardial contraction [36]. In the REF-TL simulation, the onset of the BCW occurred 38 ms after onset of left ventricular ejection, whereas for the HYP-TL simulation the delay was $28 \mathrm{~ms}$ (Fig. 3.7A). Peak wave intensity of the BCW was approximately equal for the HYP-TL simulation $\left(4.19 \cdot 10^{5}\right.$

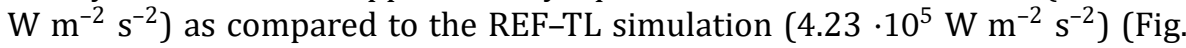
3.7A). Overall, the pattern of the simulated wave intensity tracings was similar to measured carotid arterial wave intensity tracings as reported by Hughes et al. [15] (Fig. 3.7B). 


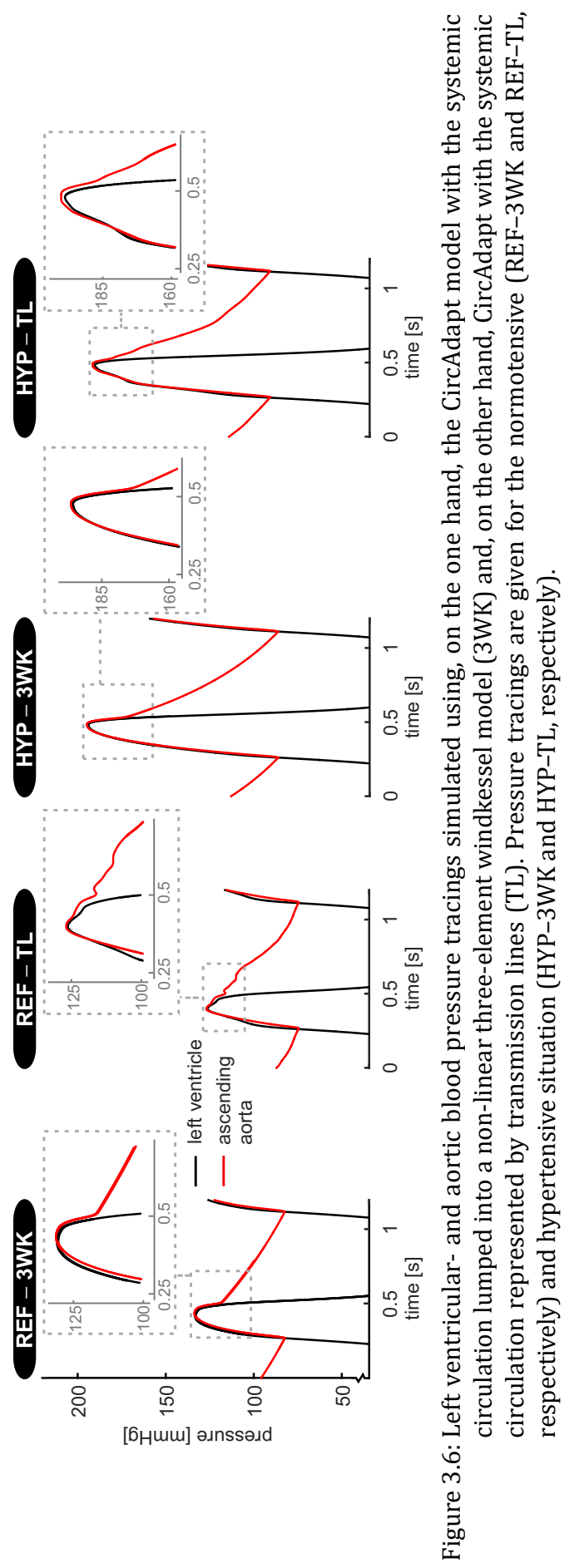




\section{A Model simulations}
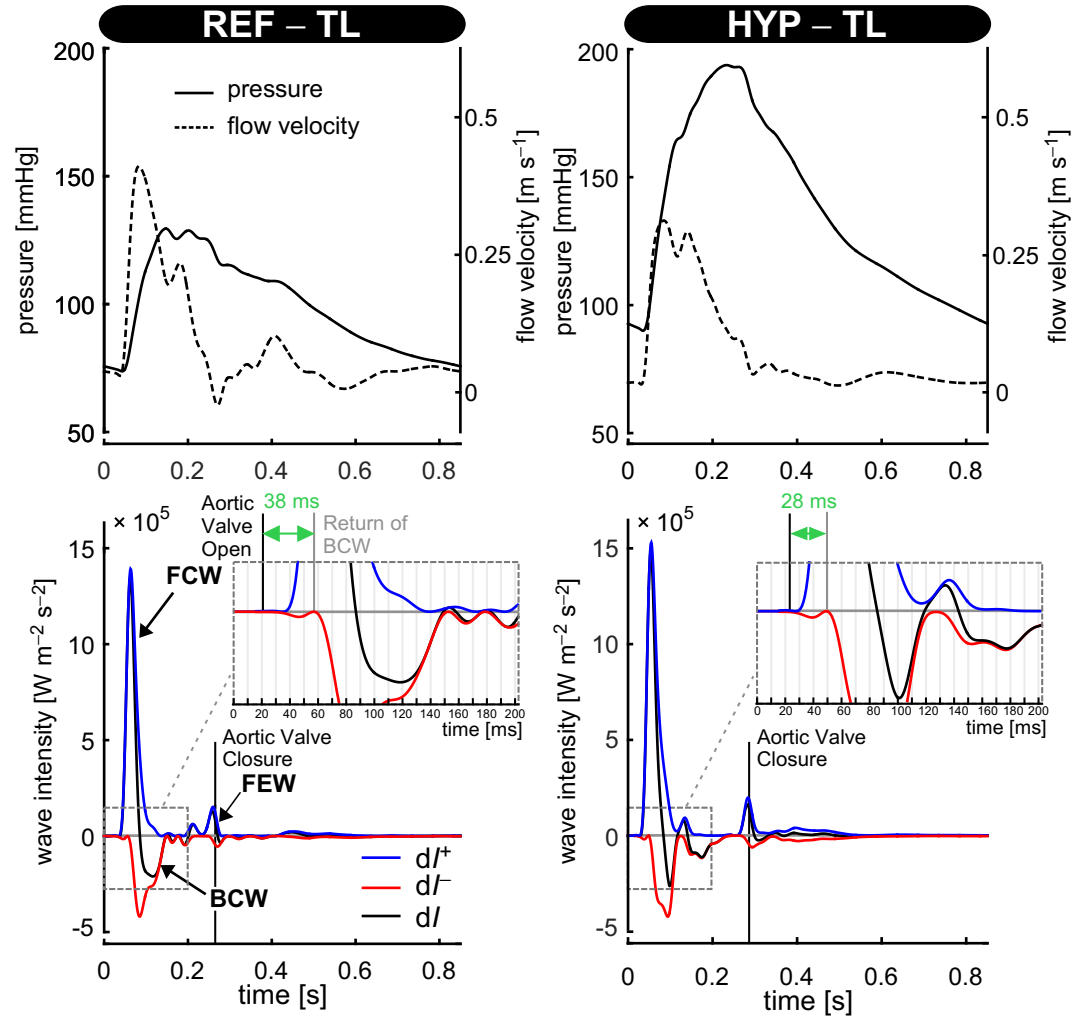

B
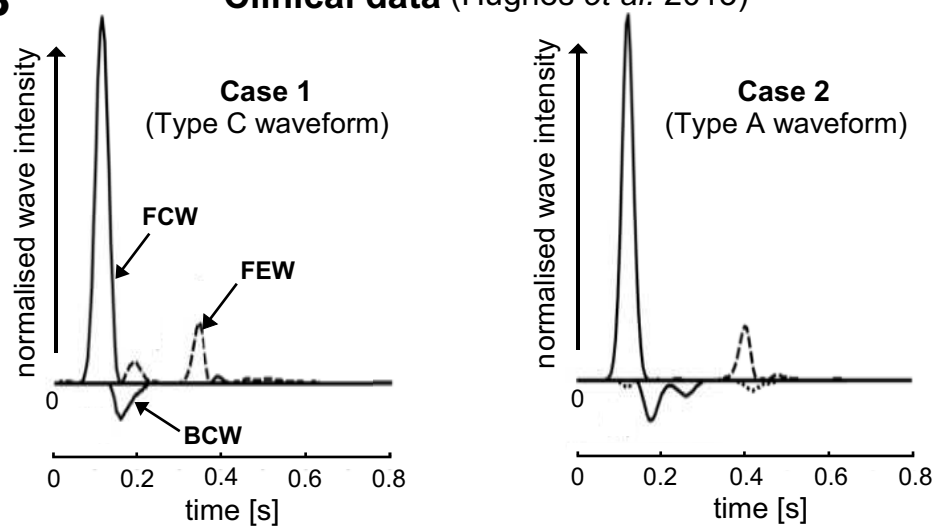

Figure 3.7: Wave intensity analysis. A: Carotid arterial pressure- and flow velocity waveforms of the reference (REF-TL) and hypertensive (HYP-TL) simulations, and corresponding wave intensity traces. B: Normalised carotid arterial wave intensity traces of two patients (adapted from [15], with reference to type C- and A-waveforms). Abbreviations: $\mathrm{d} I^{+}, \mathrm{d} I^{-}$and $\mathrm{d} I$ denote forward-, backward-, and net wave intensity, respectively. FCW, BCW, FEW: forward compression wave, backward compression wave, and forward expansion wave, respectively. 


\subsection{Discussion}

We integrated a transmission line (TL) model into the existing CircAdapt platform of whole-heart mechanics. The resulting benchmarked CircAdapt-TL model now also describes vascular wave transmission. The presented model and simulation/ validation results bring the following innovative steps forward. First, our model platform allows for computationally efficient simulation of cardiac mechanics and vascular haemodynamics. CircAdapt-TL, as implemented in MATLAB, simulates a single cardiac cycle in $6 \mathrm{~s}$, whereas the CircAdapt-3WK model requires $4 \mathrm{~s}$ but does not model wave transmission. We consider our TL implementation of a distributed model well-justified based on the acceptable increase in computational time. Second, the modular structure of CircAdapt-TL renders it easy to change the model domain (e.g. through user-defined assembly of network connections of the various modules). This versatility comes at hand when handling the model in a non-engineering environment, for example in a classroom of cardiologists in training. Third, the coupling of the extension of CircAdapt heart [25] with our framework to capture large vessels, in conjunction with modest increase in computational demand, facilitates extensive uncertainty quantification and sensitivity analysis of detailed haemodynamics mechanisms.

The field of distributed modelling of vascular wave transmission considers continuous pulse wave propagation (PWP) models that compute pressure and flow using numerical techniques such as finite differences or the method of characteristics [42], and models that use transmission line (TL) theory based on the telegraph equations $[18,20,47]$. We chose the TL model for its reduced computational cost as compared to models using the method of characteristics, its capability of operating in the time-domain, and its compatibility with the existing CircAdapt model $[20,43]$.

The validity of our TL model's numerical implementation was assessed by comparing pressure and flow waveforms generated with the TL model with those generated by the validated model of Kroon et al. [21]. For adequate comparison, an exact same model domain ranging from the large central arteries towards the smaller arteries of the left arm was chosen. Moreover, for both models boundary conditions defining proximal inflow, vessel wall mechanics, and outflow conditions were kept identical. Remaining differences in pressure and flow waveforms between both models may be attributed to the fact that in deriving the propagation constant, our TL model neglects the higher order terms to render the attenuation constant frequency-independent (Appendix 3.A1.2). This assumption is justified for high frequencies, but may become questionable for low frequencies. For the PWP model of Kroon et al. [21], such assumption was not made, since the attenuation in that model is incorporated by wall shear stress [21].

For the CircAdapt-TL model, pressure and flow waveforms of the arteries showed physiologically realistic behaviour in both time and position along the modelled domain. The morphology of arterial pressure waveforms of the aorta towards the radial and ulnar arteries resemble pressure waveforms measured during pressure catheter withdrawal from the aorta to the radial artery in man 
[38]. Venous pressure and flow waveform morphologies were similar to the ones obtained in the computational model study of Müller and Toro [30]. We found a similar venous pressure pulsatility (i.e. the maximum-minimum difference in venous pressure) as these investigations reported (i.e. between 1.2 and $2.3 \mathrm{mmHg}$ in the central veins and between 0.9 and $3.9 \mathrm{mmHg}$ in veins of the arm, respectively). In the reference (REF-TL) simulation, systolic and diastolic pressure in the aorta as well as pulse wave velocity were within normal ranges. In the hypertension (HYP-TL) simulation, blood pressure clearly increased indicated by the aortic systolic and diastolic pressures of 193 and $92 \mathrm{mmHg}$. The pulse pressure increase in the systemic hypertension simulation to $101 \mathrm{mmHg}$ is considered high given the moderate increase in pulse wave velocity to $8.0 \mathrm{~m} \mathrm{~s}^{-1}$ [28]. In our model, such large increase in pulse pressure could be caused by the fact that we did not incorporate the dilatation of arteries caused by vascular remodelling in hypertension $[17,28]$.

For the hypertension simulation, wave intensity analysis revealed earlier arrival of a backward compression wave (Fig. 3.7A), consistent with human measurements [24]. The simulated ascending aortic blood pressure waveform changed from a type $C$ waveform in the REF-TL simulation, most often seen in young adults under 30 years of age, to a type A waveform in the HYP-TL simulation, most often seen in subjects aged 40 to 65 years [31]. Although the classification of pressure waveforms according to systolic pressure augmentation may appear subtle, the clinical-epidemiological field assesses indices derived from systolic pressure augmentation for cardiovascular risk stratification [45, 32]. Simulated carotid arterial wave intensity tracings appeared similar to illustrative examples of measured tracings (Fig. 3.7B). In the HYP-TL simulation, a so-called mid-systolic forward expansion wave was present, similar to patient case 1 (around $t=0.2 \mathrm{~s}$ ) as described in [15]. Though this illustrates the level of detail possible in modelbased haemodynamic studies, the mechanistic interpretation of e.g. a mid-systolic forward expansion wave is beyond the scope of this method paper.

With lumping the systemic vessels into a $3 \mathrm{WK}$, the aortic pressure tracings lost the typical dynamics around the dicrotic notch, which were present in the REF-TL and HYP-TL simulations. Moreover, the (early) systolic pressure augmentation in the $\mathrm{LV}$ and aortic pressure tracings was absent for the pressure tracings of the REF-3WK and HYP-3WK simulation (Fig. 3.6). Given the interest in parameters such as systolic pressure augmentation, we believe that using distributed models like ours, for studies on heart-vessel interaction, may be preferable over using $3 \mathrm{WK}$ models. However, future experimental studies and clinical comparisons are needed to appraise the added value of distributed models over $3 \mathrm{WK}$ models in testing heart-vessel interaction hypotheses.

\subsubsection{Limitations}

A simplification in the TL model is that convective acceleration is neglected. However, the influence of convective acceleration on arterial pressures and flows is believed to be small [41]. Moreover, it was found that inclusion of convective 
acceleration in an arterial model domain, similar to the one used in the present study, changed pressure and flow waveforms in the various arteries only slightly (i.e. a root mean square error of $1.3 \mathrm{mmHg}$ for thoracic aortic pressure waveform and $11.3 \mathrm{ml} \mathrm{s}^{-1}$ for thoracic aortic flow waveform, respectively [39]). We expect, however, that the effect of convective acceleration will be more important when simulating exercise conditions. Therefore, in such studies the modelling error related to convective acceleration needs to be properly considered.

By excluding cerebral and coronary vessels from our model domain, we neglect the presumed influence that wave reflections and re-reflections from head and neck vessels or vessels in the myocardium may have on observed ascending aortic and carotid waveforms $[39,44]$.

Our model neither contains a skeletal-muscle pump model nor does it incorporate venous valves. Hence, the present vascular model will not account for these functional aspects with postural changes. For studies with emphasis on venous haemodynamics, the CircAdapt platform allows for a straightforward implementation of venous valves, using for instance the existing valve module as a starting point.

Like all distributed models of 1D wave transmission, our model cannot capture the complex pressure losses or local wall shear stresses when applied to disease conditions (e.g. stenosis or aneurysm). This requires either use of calibrated loss models or coupling of detailed 3D models of stenoses and aneurysms to 1D models, respectively $[5,7]$.

Reymond et al. [40] reported for the case of an apparently healthy aorta, that pressure and flow waveforms from a 3D computational fluid dynamics model and from a 1D PWP model are highly similar. The latter finding supports our notion that, in general, distributed models of wave transmission are well suited to examine and quantify heart-vessel interaction at the level of pressure and flow waveform characteristics.

\subsubsection{Perspectives}

The utility of the CircAdapt-TL model should be further tested by direct comparisons against detailed haemodynamic data from humans. We consider the concurrent use of in vivo as well as simulated data as most valuable, because both arms bring complementary assets. The model allows error free assessment of phase relationships between signals and in vivo data enables characterisation of biological and pathological variability.

In the future, we aim to further extend the CircAdapt-TL model with the cardiac adaptation module of Arts et al. [2]. This module contains a homeostatic control loop that senses offsets in mechanical load (e.g. as present in chronic hypertension), and in response, imposes geometrical (i.e. cavity volume and wall volume) adaptation of the heart. We believe that modelling cardiac adaptation is vital in assessing candidate haemodynamic indices.

Key clinical studies in this field include that of Hashimoto et al. [12]. They found a positive association between left ventricular mass, and wave reflection 
magnitude derived from pressure and flow velocity measurements, following antihypertensive treatment in left ventricular hypertrophy patients. However, a limitation of such clinical studies is that for non-invasive acquisition, pressure signals are obtained at distal measurement sites (e.g. at the radial artery) and therefore require a transfer function to obtain an estimate of the aortic pressure signal. Moreover, for clinically-gathered data, correct synchronisation of pressure and flow velocity signals is crucial, because only a small (e.g. $5 \mathrm{~ms}$ ) misalignment can cause substantial changes in derived wave (intensity) quantities [35].

\subsection{Conclusions}

We validated and incorporated a one-dimensional vascular module into the CircAdapt platform. The resulting CircAdapt-TL model enables fast simulation of whole-heart mechanics, pressure and flow waveforms at various locations along the arterial and venous systems, and allows detailed haemodynamics studies. The CircAdapt-TL model provides a valuable tool for testing hypotheses concerning heart-vessel interaction and evaluating existing haemodynamics indices. 


\section{A1 Appendix: Vascular module}

\section{A1.1 Solving strategy}

Pressure and flow in a tube constitutes of the summation of a backward (bw) and a forward (fw) wave component [9]. Therefore, at any location in the tube, it holds that

$$
\begin{aligned}
& p(z, t)=p_{\mathrm{fw}}(z-c t)+p_{\mathrm{bw}}(z+c t), \\
& q(z, t)=\frac{p_{\mathrm{fw}}(z-c t)}{Z}-\frac{p_{\mathrm{bw}}(z+c t)}{Z},
\end{aligned}
$$

with $Z$ and $c$ the characteristic Womersley number-dependent wave impedance and wave speed defined $Z=\sqrt{L\left(\alpha_{0}\right) / C}$ and $c=1 / \sqrt{L\left(\alpha_{0}\right) C}$, respectively (Section 'Derivation of the attenuation constant, wave speed and wave impedance'). Considering the proximal inlet of a tube, we assume a linear relation between pressure $p_{\text {in }}=p(0, t)$ and flow $q_{\text {in }}=q(0, t)$, characterised by a zero-flow pressure $p_{\mathrm{s}}$ and an input resistance $Z_{\text {in }}$ :

$$
p_{\text {in }}=p_{\mathrm{s}}+Z_{\text {in }} q_{\text {in }} .
$$

We derive in Section 'Derivation of source pressure' that quantities $p_{\mathrm{s}}$ and $Z_{\text {in }}$ can be calculated from $p_{\text {bw }}$ and $Z$ using the expressions given by

$$
\begin{aligned}
p_{\mathrm{s}} & =2 p_{\mathrm{bw}}, \\
Z_{\text {in }} & =Z .
\end{aligned}
$$

The proximal load relates $p_{\text {in }}$ to $q_{\text {in. }}$. Using Eq. 3.A2, $p_{\text {in }}$ and $q_{\text {in }}$ are calculated. Then, for further calculations we need to determine the amplitude of reflected wave $p_{\mathrm{fw}}$ at the entrance. It holds

$$
p_{\mathrm{fw}}=\frac{1}{2} p_{\mathrm{s}}+Z q_{\mathrm{in}} .
$$

A tube, representing a blood vessel with length $l$, has a proximal and a distal side (Fig. A1).

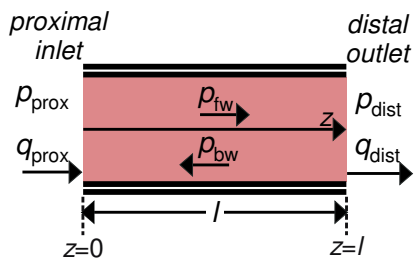

Figure A1: Overview of wave propagation in a tube segment, directed along the $z$-axis. See text for description of symbols.

For each side Eqs. 3.A1 to 3.A5 may be applied with the remark, that flow at 
the distal side is directed outward, implying that the terms with flow $q_{\text {out }}=q(l, t)$ have to change sign. At each moment in time, the amplitudes of the waves entering on both sides of the tube are calculated according to Eq. 3.A5. This wave will arrive with a delay $\tau$ later at the opposite ending. As derived by Boucher and Kitsios [9] and applied by Krus et al. [22], the contribution of wave attenuation due to friction may be lumped at the proximal and distal sides of the transmission line. Thus, for the proximal side it holds

$$
p_{\text {bw,prox }}=p_{\text {bw,dist }}(t-\tau) \exp (-\zeta l), \text { with } \tau=\frac{l}{c} .
$$

Symbols $\zeta$ and $\tau$ represent the attenuation constant and wave delay time, respectively. Attenuation constant $\zeta$ is assumed a function of the characteristic Womersley number (see Section Derivation of the attenuation constant, wave speed and wave impedance). Since $p_{\mathrm{bw} \text {,dist }}$ is calculated before, and $\tau$ is assumed to be known, $p_{\mathrm{bw}, \mathrm{prox}}$ can be calculated. Pressure $p_{\mathrm{fw}, \mathrm{dist}}$ is to be calculated similarly. When knowing these wave amplitudes, zero-flow pressure $p_{\mathrm{s}}$ and an input resistance $Z_{\text {in }}$ can be calculated on both sides of the tube, using Eqs. 3.A3 and 3.A4 and their equivalents at the distal side. Pressures and flows related to wave propagation are superimposed on DC-components of flow $q_{\mathrm{DC}}$ and pressure $p_{\mathrm{DC}}$ [9]. Furthermore, for small blood vessels, the Poiseuille resistance may become important. For the DC-pressure difference over the tube, related to $q_{\mathrm{DC}}$ it holds

$$
\Delta p_{\mathrm{DC}}=q_{\mathrm{DC}} \frac{8 \pi \eta l}{A^{2}} .
$$

Flow $q_{\mathrm{DC}}$, derived in the online supplement section 'Derivation of the DC-flow', may vary slowly, practically not influencing the behaviour of the wave. Thus, besides the wave, a tube may be subject to a (nearly) constant flow $q_{\mathrm{DC}}$, not being subject to wave attenuation. Since wave impedance $Z$ is known, we may describe the zero-flow pressures $\tilde{p}_{\text {s,prox }}$ and $\tilde{p}_{\text {s,dist }}$ by adding a DC-component to the zero-flow wave pressures $\left(p_{\mathrm{s} \text {,prox }}\right.$ and $p_{\mathrm{s} \text {,dist }}$, respectively) as calculated using Eq. 3.A3:

$$
\begin{gathered}
\tilde{p}_{\text {s,prox }}=p_{\text {s,prox }}-q_{\mathrm{DC}} Z+\frac{\Delta p_{\mathrm{DC}}}{2}, \\
\tilde{p}_{\mathrm{s}, \mathrm{dist}}=p_{\mathrm{s}, \mathrm{dist}}+q_{\mathrm{DC}} Z-\frac{\Delta p_{\mathrm{DC}}}{2} .
\end{gathered}
$$

For solving the differential equations, the solution inside is not needed; between nodes just a phase-delay and damping are imposed. However, if needed, a solution may be computed using the right- and left-ward traveling waves, attenuation constant, wave velocity, and position in the segment. Practically, if pressure and flow waveforms half-way would be required as simulation output, then the tube of interest may be subdivided into two separate tubes.

Around junctions and bifurcations, we assumed continuity of static pressure and balance of mass. 


\section{A1.2 Derivation of the attenuation constant, wave speed and wave impedance}

Previously, Huberts et al. [14] showed that for a time-domain analysis, the momentum balance equation can be represented by a lumped-parameter model consisting of a characteristic Womersley number-dependent inertance per unit length $\left(L\left(\alpha_{0}\right)\right)$ element in series with a characteristic Womersley number-dependent resistor per unit length $\left(R\left(\alpha_{0}\right)\right)$ element. We define the characteristic Womersley number $\left(\alpha_{0}\right)$ as $\alpha_{0}=r_{0} \sqrt{\rho \omega_{0} / \eta}$, with $\omega_{0}$ the characteristic angular frequency, $r_{0}$ the reference lumen radius, and constants $\rho$ and $\eta$ the blood density and blood dynamic viscosity, respectively. For our time-domain approach, we adopted the method of Huberts et al. [14]. Expressions for $R\left(\alpha_{0}\right)$ and $L\left(\alpha_{0}\right)$ are given by

$$
\begin{aligned}
& L\left(\alpha_{0}\right)=\overbrace{\left(\frac{1}{2-c_{p}\left(\alpha_{0}\right)}\right)}^{g\left(\alpha_{0}\right)} L_{0}, \\
& R\left(\alpha_{0}\right)=\overbrace{\left(\frac{c_{q}\left(\alpha_{0}\right)}{2-\alpha_{p}\left(\alpha_{0}\right)}\right)}^{\left.2-\alpha_{0}\right)} R_{0} .
\end{aligned}
$$

Here, inertance per unit length $\left(L_{0}\right)$ and Poiseuille resistance per unit length $\left(R_{0}\right)$ are defined $L_{0}=\rho / A_{0}$ and $R_{0}=8 \pi \eta / A_{0}^{2}$, respectively. The coefficients $c_{p}$ and $c_{q}$ were derived by Bessems et al. [6]:

$$
\begin{aligned}
& c_{p}\left(\alpha_{0}\right)= \begin{cases}1+\frac{\sqrt{2}}{\alpha_{0}}\left(1-\frac{\sqrt{2}}{2 \alpha_{0}}\right) & \text { if } \alpha_{0}>\sqrt{2} \\
\frac{3}{2} & \text { if } \alpha_{0} \leq \sqrt{2}\end{cases} \\
& c_{q}\left(\alpha_{0}\right)= \begin{cases}\frac{\alpha_{0}}{4 \sqrt{2}}\left(1-\frac{\sqrt{2}}{2 \alpha_{0}}\right)^{-1} & \text { if } \alpha_{0}>\sqrt{2} \\
\frac{1}{2} & \text { if } \alpha_{0} \leq \sqrt{2}\end{cases}
\end{aligned}
$$

In Fig. A2, $g$ and $h$ are depicted as a function of $\alpha_{0}$. The ranges chosen for $\alpha_{0}$, i.e. $[0, \sqrt{2}]$ and $[\sqrt{2}, \infty]$ render that for the limiting cases of $\alpha_{0}=0$ and $\alpha_{0} \rightarrow \infty$, the friction term coincides with Womersley theory [6]. 


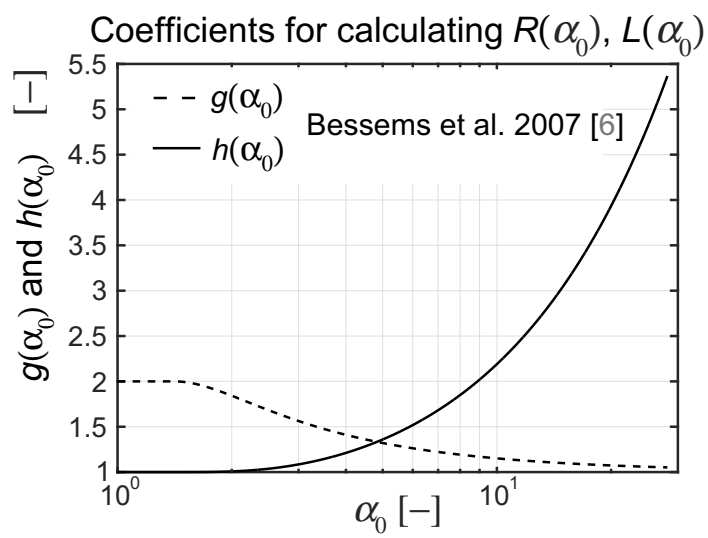

Figure A2: Coefficients $g$ and $h$ as a function of the characteristic Womersley number $\left(\alpha_{0}\right)$, used for calculating the characteristic Womersley number dependent resistance $R\left(\alpha_{0}\right)$ and inertance $L\left(\alpha_{0}\right)$, respectively.

Using the expressions for $R\left(\alpha_{0}\right)$ and $L\left(\alpha_{0}\right)$, attenuation constant $\zeta$ is obtained by calculating propagation constant $\gamma$, evaluated for characteristic frequency $\omega_{0}$. We approximated $\gamma$ following Matick [27]:

$$
\begin{aligned}
& \gamma=\zeta+j \beta=j \omega \sqrt{L\left(\alpha_{0}\right) C} \sqrt{1-\frac{j R\left(\alpha_{0}\right)}{\omega L\left(\alpha_{0}\right)}} \\
& \approx j \omega \sqrt{L\left(\alpha_{0}\right) C}\left(1-\frac{j R\left(\alpha_{0}\right)}{2 \omega L\left(\alpha_{0}\right)}+\mathscr{O}\left(\frac{1}{\omega^{2}}\right)\right) \\
& \approx \frac{R\left(\alpha_{0}\right)}{2 \sqrt{L\left(\alpha_{0}\right) / C}}+j \omega \sqrt{L\left(\alpha_{0}\right) C},
\end{aligned}
$$

with $\zeta$ the attenuation constant per unit length, $\beta$ the phase constant per unit length and $C$ the compliance per unit length. The characteristic Womersley number-dependent wave speed $c$ was calculated using

$$
c=\frac{\omega}{\beta}=\frac{1}{\sqrt{L\left(\alpha_{0}\right) C}} .
$$

Finally, Womersley number-dependent wave impedance was calculated using

$$
Z=\sqrt{\frac{L\left(\alpha_{0}\right)}{C}} .
$$




\section{A1.3 Derivation of source pressure}

As previously described, it holds for pressure and flow at any location that

$$
\begin{aligned}
& p(z, t)=p_{\mathrm{fw}}(z-c t)+p_{\mathrm{bw}}(z+c t), \\
& q(z, t)=\frac{p_{\mathrm{fw}}(z-c t)}{Z}-\frac{p_{\mathrm{bw}}(z+c t)}{Z} .
\end{aligned}
$$

Pressure and flow at the proximal side of the tube are given by

$$
\begin{aligned}
& p_{\text {prox }}=p_{\text {fw,prox }}+p_{\text {bw,prox }}, \\
& q_{\text {prox }}=\frac{p_{\mathrm{fw}, \text { prox }}}{Z_{\text {prox }}}-\frac{p_{\text {bw,prox }}}{Z_{\text {prox }}} .
\end{aligned}
$$

If $q_{\text {prox }}=0$ and $p_{\text {bw,prox }}$ is known, then for proximal source pressure $p_{\text {s,prox }}=p_{\text {prox }}$ it follows after substitution of Eq. 3.A16:

$$
p_{\text {s,prox }}=2 p_{\text {bw,prox }} \text {. }
$$

For short circuit flow $q_{\mathrm{s} \text {,prox }}=q_{\text {prox }}$ with $p_{\text {prox }}=0$, it follows after substitution of Eq. 3.A16:

$$
q_{\text {s,prox }}=\frac{2 p_{\text {bw,prox }}}{Z_{\text {prox }}} .
$$

The source impedance of the proximal side of the tube is calculated using

$$
Z_{\text {prox }}=\frac{p_{\text {s,prox }}}{q_{\text {s,prox }}}=Z .
$$

Finally, the applied equations for the distal side of the tube are given by

$$
\begin{aligned}
p_{\mathrm{s}, \mathrm{dist}} & =2 p_{\mathrm{fw}, \mathrm{dist}}, \\
q_{\mathrm{s}, \mathrm{dist}} & =\frac{2 p_{\mathrm{fw}, \mathrm{dist}}}{Z_{\mathrm{dist}}}, \\
Z_{\mathrm{dist}} & =\frac{p_{\mathrm{s}, \mathrm{dist}}}{q_{\mathrm{s}, \mathrm{dist}}}=Z .
\end{aligned}
$$

\section{A1.4 Derivation of the DC-flow}

The DC-flow ( $q_{\mathrm{DC}}$ ), averaged over the tube length, changes due to contribution of flow waves entering and leaving from the proximal and distal side. In case no wave enters, the waves inside the tube will disappear completely after wave delay time $\tau$. Using Eq. 3.A8, reaching of the DC-steady state (i.e. term B of Eq. 3.A21 becoming zero) was approximated by a first order differential equation with time 
constant equal to $\tau$ :

$$
\frac{d q_{\mathrm{DC}}}{d t}=\overbrace{\left(\frac{p_{\mathrm{s}, \mathrm{dist}}-p_{\mathrm{s}, \text { prox }}}{2 Z}-q_{\mathrm{DC}}\right)}^{B} \frac{1}{\tau} .
$$

\section{A2 Benchmark comparison between TL and PWP model}

For the benchmark comparison, we implemented a network of tubes describing the central arteries (i.e. the aorta, carotid artery, and vertebral artery), as well as the arteries of the left arm (Fig. A3).

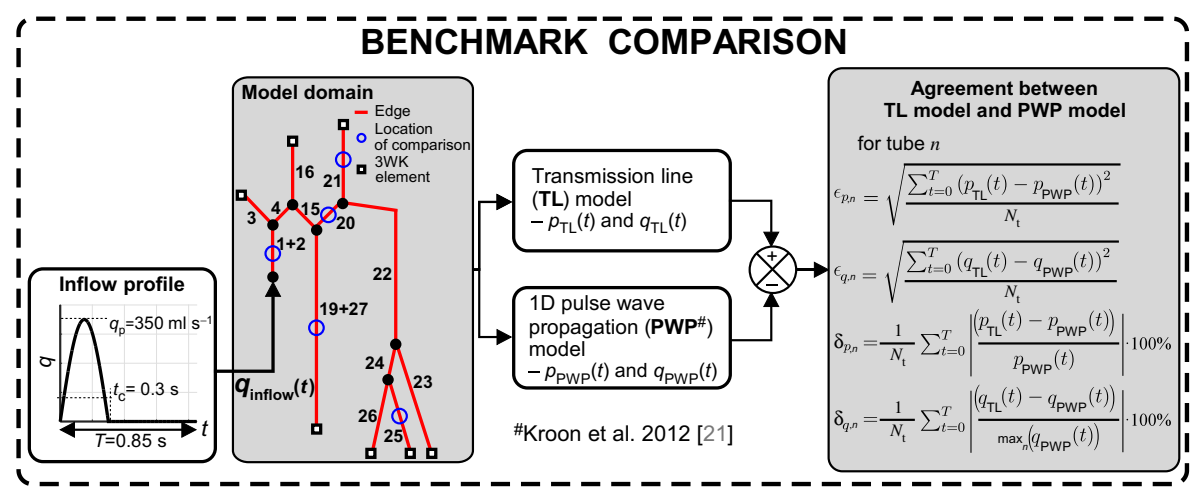

Figure A3: Benchmark comparison of the transmission line (TL) model with the previously validated 1D pulse wave propagation (PWP) model. We implemented a network of 13 tubes representing the large central arteries and the arteries of the left arm. Geometrical and mechanical properties of arterial segments that were used in the benchmark comparison are given in Table A2. A half-sinusoidal inflow profile $\left(q_{\text {inflow }}\right)$ was prescribed to the ascending aorta. Boundary conditions; proximal inflow, termination of distal vessels using a non-linear three element winkessel model ( $3 \mathrm{WK})$, vessel geometry and material properties were kept the same for the TL model and PWP model. Evaluated agreement metrics were root mean square errors $\epsilon_{p}$ and $\epsilon_{q}$, as well as the relative errors $\delta_{p}$ and $\delta_{q}$ Furthermore, $N_{\mathrm{t}}$ denotes the number of time points for which a comparison was made. A graphical comparison between pressure and flow waveforms is given for five tubes (blue circles).

Geometrical and mechanical properties of all tubes were based on Reymond et al. [39], (Table A2). Vessel stiffness coefficient ( $k$ ) for the aortic segments, vertebral artery and carotid artery was estimated by fitting the constitutive law to in vitro data from Hayashi et al. [13]. For the radial, ulnar and interosseous arteries, stiffness was assumed equal to vertebral artery stiffness. For the subclavian-axillarybrachial arterial segment, stiffness was taken as the mean of aortic and carotid 
artery stiffness (Table A2). Flow fractions were based on previously published patient measurements with duplex ultrasound and phase-contrast magnetic resonance imaging [14]. For the benchmark comparison, we defined a half-sinusoidal inflow profile as a proximal inlet boundary condition $\left(q_{\text {inflow }}(t)\right)$ :

$$
q_{\text {inflow }}(t)= \begin{cases}q_{\mathrm{p}} \sin \left(\frac{\pi t}{t_{\mathrm{c}}}\right) & \text { if } 0 \leq t \leq t_{\mathrm{c}} \\ 0 & \text { if } t>t_{\mathrm{c}} .\end{cases}
$$

Distal tubes were terminated using $3 \mathrm{WKs}$. For these simulations, time step $(\Delta t)$ was kept at $0.5 \mathrm{~ms}$, duty cycle $\left(t_{\mathrm{c}}\right)$ at $0.3 \mathrm{~s}$, cardiac cycle duration $(T)$ at $0.85 \mathrm{~s}$, and peak flow rate $\left(q_{\mathrm{p}}\right)$ at $350 \mathrm{ml} \mathrm{s}^{-1}$, resulting in a physiological cardiac output. Boundary conditions were kept equal for the TL model and PWP model. Reference pressure $\left(p_{0}\right)$ was kept at $105 \mathrm{mmHg}$, external pressure $\left(p_{\text {ext }}\right)$ at $0 \mathrm{mmHg}$ and outflow pressure at $4 \mathrm{mmHg}$. We chose an element size of $0.01 \mathrm{~m}$. Convergence of the calculated haemodynamics was evaluated after each simulated cardiac cycle. Hereto, the calculated nodal pressures, $p(t)$, of the current cycle $\left(i_{\text {cyc }}\right)$ were compared to those of the previous cycle $\left(i_{\text {cyc }}-1\right)$. An expression for the haemodynamics convergence norm $\left(\epsilon_{\text {norm }}\right)$ is given as a relative root mean square:

$$
\epsilon_{\mathrm{norm}}=\max _{n}\left(\sqrt{\frac{\sum_{t=0}^{T}\left(p_{n, i_{\mathrm{cyc}}}^{t}-p_{n, i_{\mathrm{cyc}}-1}^{t}\right)^{2}}{\sum_{t=0}^{T}\left(p_{n, i_{\mathrm{cyc}}-1}^{t}\right)^{2}}}\right),
$$

with $n$ referring to the tube number as given in Table A2. Throughout simulations, the haemodynamics convergence criterion was kept at $10^{-2}$. Agreement between $p$ and $q$ waveforms, expressed by root mean square errors $\epsilon_{p}$ and $\epsilon_{q}$, as well as relative errors $\delta_{p}$ and $\delta_{q}$, was calculated for the centre element of each tube (Fig. A3).

\section{A2.1 Numerical implementation of the pulse wave propagation model of Kroon et al. [21]}

For the 1D vessels of the PWP model, we assumed the same constitutive law as used for the TL model. Moreover for the periphery, we implemented the same nonlinear three-element windkessel as used for the TL model. Coupling and solving of $1 \mathrm{D}$ mass conservation and momentum balance equations (after neglecting the convective acceleration term) and the $0 \mathrm{D}$ equation for pressure and flow in the periphery was performed using the method previously published by Kroon et al. [21]. We used a simplified trapezoidal scheme for spatial discretisation. The second-order backward difference scheme was used for time discretisation. 


\section{A3 Simulations performed using the CircAdapt-3WK model}

As described in the main text of this article, we additionally performed referenceand hypertension simulations using the CircAdapt model with the systemic circulation lumped into the non-linear three-element windkessel model (REF-3WK and HYP-3WK, respectively). For the REF-3WK simulation, reference pressure was $105 \mathrm{mmHg}$ and vessel stiffness coefficient $(k)$ was kept equal to 8 for the arterial side and 10 for the venous side, respectively. For the HYP-3WK simulation, reference pressure was increased to $135 \mathrm{mmHg}$ and the $k$-value of the systemic arterial 3WK was set to 14. For both simulations, characteristic vessel bed length $\left(l_{\mathrm{AV}}\right)$ was chosen $0.8 \mathrm{~m} \mathrm{[46].}$

\section{A4 Code availability and calculation loop of the CircAdapt-TL model}

The CircAdapt-TL model source code can be retrieved from https://github. com/Mheu1991/CircAdaptTL/. Table A1 refers to specific lines of MATLAB-code providing programmatic logic of the aforementioned relations (i.e. Eq A\#) that were implemented to solve our TL model. Furthermore, Fig. A4 illustrates the calculation loop in CircAdapt-TL.

Table A1: Index linking mathematical relations of the transmission line model to MATLABscripts and underlying lines of code. Previous publications comprising existing CircAdapt modules are indicated.

\begin{tabular}{ccc}
\hline Equation & MATLAB-script & Line no. \\
\hline A5 & PNodeVDot.m & 52 \\
A6 & TubeDelays.m & $36,37,85,86$ \\
A7 & TubeV2p.m & 51,52 \\
A8 & TubeDelays.m & 60 to 63 \\
A9 & CalcApproximateVelocityProfile.m & 29 \\
A10 & CalcApproximateVelocityProfile.m & 30 \\
A11 & CalcApproximateVelocityProfile.m & 27,28 \\
A12 & CalcApproximateVelocityProfile.m & 36 \\
A13 & CalcApproximateVelocityProfile.m & 37 \\
A14 & CalcApproximateVelocityProfile.m & 38 \\
A21 & TubeDelays.m & 57 to 59 \\
\hline
\end{tabular}




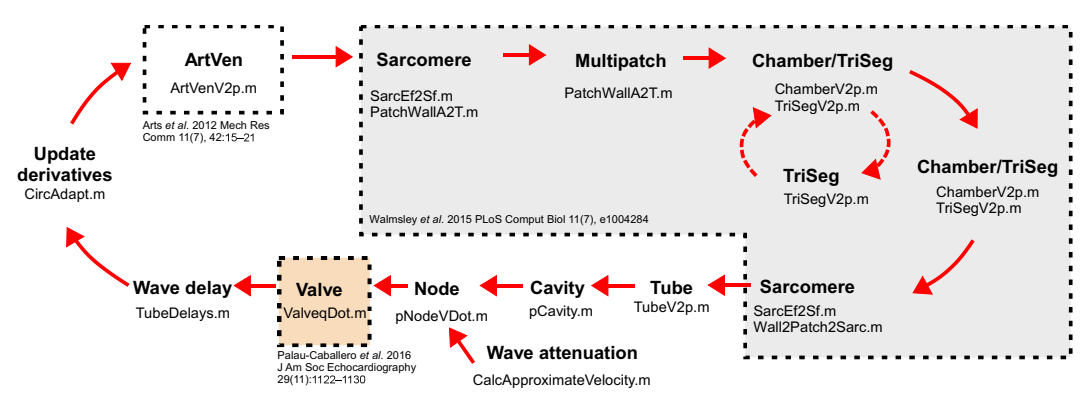

Figure A4: Calculation loop of the CircAdapt-TL model.

\section{A5 Calculation of wave intensity}

The fundamental basis of wave intensity analysis is described in Parker [35] Briefly, at a fixed measurement location, waves are considered incremental fronts for which the sum of successive forward- or backward travelling wavefronts form the measured pressure- or flow velocity waveform. Wave intensity analysis determines the magnitude and direction of wavefronts from derivatives of local pressure and flow velocity [35]. Wave intensity $(\mathrm{d} I)$ is here calculated using the time derivatives of pressure $(\mathrm{d} p / \mathrm{d} t)$ and flow velocity $(\mathrm{d} U / \mathrm{d} t)$ :

$$
\mathrm{d} I=\frac{\mathrm{d} p}{\mathrm{~d} t} \frac{\mathrm{d} U}{\mathrm{~d} t} .
$$

Wave intensity is positive when forward travelling waves are dominant, whereas it is negative when backward travelling waves are dominant. Wave intensity itself may also be calculated for forward- and backward waves. This requires separation of the simulated pressure and flow velocity waveforms into their respective forward- and backward components. By assuming balance of mass and momentum in elastic tubes and neglecting viscous friction, a simple relation between the time derivatives of pressure and flow velocity is given by [35]

$$
\frac{\mathrm{d} p}{\mathrm{~d} t}=\rho c \frac{\mathrm{d} U}{\mathrm{~d} t}
$$

with $c$ the local pulse wave velocity. Parker [35] showed that the relation as given in Eq. 3.A25 may be written for forward- and backward components as

$$
\begin{aligned}
\frac{\mathrm{d} p^{+}}{\mathrm{d} t} & =\rho c \frac{\mathrm{d} U^{+}}{\mathrm{d} t}, \text { and } \\
\frac{\mathrm{d} p^{-}}{\mathrm{d} t} & =-\rho c \frac{\mathrm{d} U^{-}}{\mathrm{d} t},
\end{aligned}
$$

with superscripts "+", and "-" the forward- and backward wave components. Because we assumed that summation of backward- and forward waves yields the 
measured pressure and flow velocity waveforms, the aforementioned equation may be expressed as [35]

$$
\begin{aligned}
\frac{\mathrm{d} p^{+}}{\mathrm{d} t} & =\left(\frac{\mathrm{d} p}{\mathrm{~d} t}+\rho c \frac{\mathrm{d} U}{\mathrm{~d} t}\right) / 2, \\
\frac{\mathrm{d} p^{-}}{\mathrm{d} t} & =\left(\frac{\mathrm{d} p}{\mathrm{~d} t}-\rho c \frac{\mathrm{d} U}{\mathrm{~d} t}\right) / 2, \\
\frac{\mathrm{d} U^{+}}{\mathrm{d} t} & =\left(\frac{\mathrm{d} U}{\mathrm{~d} t}+\frac{\mathrm{d} p}{\mathrm{~d} t} / \rho c\right) / 2, \text { and } \\
\frac{\mathrm{d} U^{-}}{\mathrm{d} t} & =\left(\frac{\mathrm{d} U}{\mathrm{~d} t}-\frac{\mathrm{d} p}{\mathrm{~d} t} / \rho c\right) / 2,
\end{aligned}
$$

Using Eq. 3.A24, intensity for forward- and backward waves, and the net pressure wave is expressed by

$$
\begin{aligned}
\mathrm{d} I^{+} & =\frac{\mathrm{d} p^{+}}{\mathrm{d} t} \frac{\mathrm{d} U^{+}}{\mathrm{d} t}, \\
\mathrm{~d} I^{-} & =\frac{\mathrm{d} p^{-}}{\mathrm{d} t} \frac{\mathrm{d} U^{-}}{\mathrm{d} t}, \text { and } \\
\mathrm{d} I & =\mathrm{d} I^{+}+\mathrm{d} I^{-}
\end{aligned}
$$

For mathematical derivation of Eqs. 3.A27 and 3.A28, we refer to the work of Parker [35]. It should be noted that whereas Parker [35] calculated wave intensity based on instantaneous differences in pressure and flow velocity (i.e. $\mathrm{d} p$ and $\mathrm{d} U$ ), we calculated time derivatives (i.e. $\mathrm{d} p / \mathrm{d} t$ and $\mathrm{d} U / \mathrm{d} t$ ). As a result, wave intensity is given in units of $\left[\mathrm{W} \mathrm{m}^{-2} \mathrm{~s}^{-2}\right]$, instead of in $\left[\mathrm{W} \mathrm{m}^{-2}\right]$. 


\section{A6 Description of geometrical and mechanical parameters of the implemented network of arteries and veins}

Table A2: Geometrical and mechanical properties of arterial segments, based on Reymond et al. [39]. The italic $q_{\mathrm{AV}}$ values represent the terminal flow (here expressed as a percentage of cardiac output) as used in the benchmark comparison. The italic tube numbers (e.g. 3) refer to segments that were also used in the benchmark comparison.

\begin{tabular}{|c|c|c|c|c|c|c|}
\hline tube & name [-] & $l[\mathrm{~m}]$ & $d_{\text {prox }}[\mathrm{mm}]$ & $d_{\text {dist }}[\mathrm{mm}]$ & $k[-]$ & $q_{\mathrm{AV}}[\%]$ \\
\hline 1 & ascending aorta 1 & 0.020 & 29.4 & 29.3 & 10 & \\
\hline 2 & ascending aorta 2 & 0.020 & 29.3 & 28.8 & 10 & \\
\hline 3 & brachiocephalic artery & 0.040 & 20.2 & 18.0 & 10 & 20 \\
\hline 4 & aortic $\operatorname{arch} \mathrm{A}$ & 0.020 & 25.1 & 24.0 & 10 & \\
\hline 5 & r. subclavian artery & 0.040 & 11.5 & 9.0 & 10 & \\
\hline 6 & r. common carotid artery & 0.100 & 13.5 & 7.0 & 14 & \\
\hline 7 & r. vertebral artery & 0.150 & 3.7 & 2.8 & 16 & 5 \\
\hline 8 & r. subcl. B + axill. + brach. & 0.430 & 8.1 & 4.7 & 12 & \\
\hline 9 & r. radial artery & 0.240 & 3.7 & 3.1 & 12 & 2.3 \\
\hline 10 & r. ulnar artery A & 0.070 & 3.7 & 3.4 & 16 & \\
\hline 11 & r. interosseous artery & 0.080 & 2.1 & 1.8 & 16 & 0.14 \\
\hline 12 & r. ulnar artery B & 0.180 & 3.2 & 2.8 & 16 & 2.3 \\
\hline 13 & r. internal carotid artery & 0.180 & 5.7 & 4.3 & 16 & 6 \\
\hline 14 & r. external carotid artery & 0.050 & 5.0 & 4.5 & 14 & 2 \\
\hline 15 & aortic $\operatorname{arch} B$ & 0.040 & 21.4 & 20.8 & 14 & \\
\hline 16 & l. common carotid artery & 0.140 & 12.0 & 6.0 & 10 & 15 \\
\hline 17 & l. internal carotid artery & 0.180 & 5.3 & 4.1 & 14 & 6 \\
\hline 18 & 1. external carotid artery & 0.050 & 4.7 & 4.3 & 14 & 2 \\
\hline 19 & thoracic aorta $A$ & 0.060 & 20.0 & 18.9 & 10 & \\
\hline 20 & 1. subclavian artery A & 0.040 & 11.0 & 8.5 & 12 & \\
\hline 21 & l. vertebral artery & 0.150 & 3.7 & 2.8 & 16 & 5,5 \\
\hline 22 & l. subcl. B + axill. + brach. & 0.430 & 8.1 & 4.7 & 10 & \\
\hline 23 & 1. radial artery & 0.240 & 3.5 & 2.8 & 16 & $2.3,2$ \\
\hline 24 & 1. ulnar artery $\mathrm{A}$ & 0.070 & 4.3 & 4.3 & 16 & \\
\hline 25 & l. interosseous artery & 0.079 & 1.8 & 1.8 & 16 & $0.14,2$ \\
\hline 26 & 1. ulnar artery B & 0.180 & 4.1 & 3.7 & 16 & $2.3,2$ \\
\hline 27 & thoracic aorta B & 0.110 & 16.5 & 12.9 & 10 & 54 \\
\hline 28 & abdominal aorta $\mathrm{A}$ & 0.060 & 12.2 & 12.2 & 10 & \\
\hline 29 & celiac artery & 0.040 & 7.8 & 6.9 & 12 & \\
\hline 30 & hepatic artery & 0.070 & 5.4 & 4.4 & 12 & 3.3 \\
\hline 31 & gastric artery & 0.080 & 3.2 & 3.0 & 12 & 2.2 \\
\hline 32 & splenic artery & 0.070 & 4.2 & 3.9 & 12 & 5.2 \\
\hline 33 & superior mesenteric artery & 0.060 & 7.9 & 7.1 & 12 & 13 \\
\hline
\end{tabular}




\begin{tabular}{lcccccc}
34 & abdominal aorta B & 0.020 & 11.5 & 11.8 & 10 & \\
35 & l. renal artery & 0.040 & 5.2 & 5.2 & 10 & 10.7 \\
36 & abdominal aorta C & 0.020 & 11.8 & 11.0 & 10 & \\
37 & r. renal artery & 0.040 & 5.2 & 5.2 & 12 & 10.7 \\
38 & abdominal aorta D & 0.110 & 11.6 & 7.0 & 10 & \\
39 & inferior mesenteric artery & 0.050 & 4.7 & 3.2 & 12 & 1.8 \\
40 & abdominal aorta E & 0.020 & 10.8 & 6.1 & 10 & \\
41 & r. common iliac artery & 0.060 & 7.9 & 4.0 & 16 & \\
42 & 1. common iliac artery & 0.060 & 7.9 & 4.0 & 16 & \\
43 & l. external iliac artery & 0.150 & 6.4 & 3.7 & 16 & \\
44 & l. internal iliac artery & 0.050 & 4.0 & 2.8 & 16 & 1.5 \\
45 & l. femoral artery & 0.443 & 5.2 & 2.3 & 30 & \\
46 & l. deep femoral artery & 0.126 & 4.0 & 3.7 & 30 & 1.5 \\
47 & l. posterior tibial artery & 0.321 & 3.1 & 2.8 & 30 & 2.5 \\
48 & l. anterior tibial artery & 0.343 & 2.6 & 2.3 & 30 & 2.5 \\
49 & r. external iliac artery & 0.144 & 6.4 & 3.7 & 30 & \\
50 & r. internal iliac artery & 0.050 & 4.0 & 2.8 & 30 & 2.5 \\
51 & r. femoral artery & 0.450 & 5.2 & 2.3 & 30 & \\
52 & r. deep femoral artery & 0.130 & 4.0 & 3.7 & 30 & 2.1 \\
53 & r. posterior tibial artery & 0.330 & 3.1 & 2.8 & 30 & 2.5 \\
54 & r. anterior tibial artery & 0.350 & 2.6 & 2.3 & 30 & 2.2 \\
& & & & & \\
\hline
\end{tabular}


Table A3: Geometrical and mechanical properties of venous segments, based on Müller and Toro [30].

\begin{tabular}{|c|c|c|c|c|c|}
\hline tube & name [-] & $l[\mathrm{~m}]$ & $d_{\text {prox }}[\mathrm{mm}]$ & $d_{\text {dist }}[\mathrm{mm}]$ & $k[-]$ \\
\hline 55 & vena cava superior & 0.035 & 16.0 & 16.0 & 10 \\
\hline 56 & r. brachiocephalic vein & 0.040 & 11.3 & 11.3 & 10 \\
\hline 57 & r. internal carotid vein & 0.025 & 5.0 & 5.0 & 10 \\
\hline 58 & r. external carotid vein & 0.025 & 5.0 & 5.0 & 10 \\
\hline 59 & r. subclavian vein $\mathrm{A}$ & 0.030 & 11.3 & 11.3 & 10 \\
\hline 60 & r. vertebral vein & 0.110 & 3.2 & 3.2 & 10 \\
\hline 61 & r. subclavian vein $B$ & 0.030 & 10.4 & 10.4 & 10 \\
\hline 62 & r. subclavian vein $\mathrm{C}$ & 0.270 & 10.4 & 10.4 & 10 \\
\hline 63 & r. radial vein & 0.406 & 4.0 & 4.0 & 10 \\
\hline 64 & r. ulnar vein $A$ & 0.100 & 4.0 & 4.0 & 10 \\
\hline 65 & r. interosseous vein & 0.070 & 2.0 & 2.0 & 10 \\
\hline 66 & r. ulnar vein $B$ & 0.306 & 4.0 & 4.0 & 10 \\
\hline 67 & 1. brachiocephalic vein & 0.075 & 10.9 & 10.9 & 10 \\
\hline 68 & l. internal carotid vein & 0.025 & 5.0 & 5.0 & 10 \\
\hline 69 & l. external carotid vein & 0.025 & 5.0 & 5.0 & 10 \\
\hline 70 & 1. subclavian vein $A$ & 0.030 & 11.2 & 11.2 & 10 \\
\hline 71 & l. vertebral vein & 0.110 & 3.2 & 3.2 & 10 \\
\hline 72 & 1. subclavian vein B & 0.030 & 10.4 & 10.4 & 10 \\
\hline 73 & l. subclavian vein $C$ & 0.270 & 10.4 & 10.4 & 10 \\
\hline 74 & 1. radial vein & 0.406 & 4.0 & 4.0 & 10 \\
\hline 75 & 1. ulnar vein $\mathrm{A}$ & 0.100 & 4.0 & 4.0 & 10 \\
\hline 76 & 1. interosseous vein & 0.070 & 2.0 & 2.0 & 10 \\
\hline 77 & l. ulnar vein B & 0.306 & 4.0 & 4.0 & 10 \\
\hline 78 & vena cava inferior & 0.020 & 15.2 & 15.2 & 10 \\
\hline 79 & celiac vein & 0.040 & 9.8 & 9.8 & 10 \\
\hline 80 & gastric vein & 0.068 & 9.8 & 9.8 & 10 \\
\hline 81 & hepatic vein & 0.068 & 9.8 & 9.8 & 10 \\
\hline 82 & splenic vein & 0.068 & 9.8 & 9.8 & 10 \\
\hline 83 & superior mesenteric vein & 0.068 & 9.8 & 9.8 & 10 \\
\hline 84 & vena cava inferior $B$ & 0.015 & 15.2 & 15.2 & 10 \\
\hline 85 & 1. renal vein & 0.032 & 5.0 & 5.0 & 10 \\
\hline 86 & r.renal vein & 0.032 & 5.0 & 5.0 & 10 \\
\hline 87 & vena cava inferior $\mathrm{C}$ & 0.015 & 15.2 & 15.2 & 10 \\
\hline 88 & vena cava inferior D & 0.125 & 15.2 & 15.2 & 10 \\
\hline 89 & inferior mesenteric vein & 0.060 & 9.0 & 9.0 & 10 \\
\hline 90 & vena cava inferior $\mathrm{E}$ & 0.080 & 15.2 & 15.2 & 10 \\
\hline 91 & r. common iliac vein A & 0.038 & 11.6 & 11.6 & 10 \\
\hline 92 & r. common iliac vein B & 0.020 & 11.6 & 11.6 & 10 \\
\hline 93 & r. inner iliac vein & 0.050 & 3.0 & 3.0 & 10 \\
\hline 94 & r. external iliac vein & 0.144 & 6.0 & 5.0 & 10 \\
\hline 95 & r. deep femoral vein & 0.126 & 7.0 & 7.0 & 10 \\
\hline
\end{tabular}




\begin{tabular}{cccccc}
96 & r. femoral vein & 0.254 & 7.0 & 7.0 & 10 \\
97 & r. posterior tibial vein & 0.173 & 3.0 & 3.0 & 10 \\
98 & r. anterior tibial vein & 0.173 & 3.0 & 3.0 & 10 \\
99 & l. common iliac vein A & 0.038 & 11.6 & 11.6 & 10 \\
100 & l. common iliac vein B & 0.020 & 11.6 & 11.6 & 10 \\
101 & l. inner iliac vein & 0.050 & 3.0 & 3.0 & 10 \\
102 & l. external iliac vein & 0.144 & 10.0 & 10.0 & 10 \\
103 & l. deep femoral vein & 0.126 & 7.0 & 7.0 & 10 \\
104 & l. femoral vein & 0.254 & 7.0 & 7.0 & 10 \\
105 & l. posterior tibial vein & 0.173 & 3.0 & 3.0 & 10 \\
106 & l. anterior tibial vein & 0.173 & 3.0 & 3.0 & 10 \\
& & & & & \\
\hline
\end{tabular}




\section{References}

[1] T. Arts, P. H. M. Bovendeerd, F. W. Prinzen, and R. S. Reneman. Relation between left ventricular cavity pressure and volume and systolic fiber stress and strain in the wall. Biophysical Journal, 59(1):93-102, 1991.

[2] T. Arts, J. Lumens, W. Kroon, and T. Delhaas. Control of whole heart geometry by intramyocardial mechano-feedback: a model study. PLOS Computational Biology, 8(2):e1002369, 2012.

[3] T. Arts, K. Reesink, W. Kroon, and T. Delhaas. Simulation of adaptation of blood vessel geometry to flow and pressure: Implications for arterio-venous impedance. Mechanics Research Communications, 42:15-21, 2012.

[4] S. Bassez, P. Flaud, and M. Chauveau. Modeling of the deformation of flexible tubes using a single law: application to veins of the lower limb in man. Journal of Biomechanical Engineering, 123(1): 58-65, 2001.

[5] D. Bessems. On the propagation of pressure and flow waves through the patient specific arterial system. PhD thesis, Eindhoven University of Technology, 2007.

[6] D. Bessems, M. C. M. Rutten, and F. N. van de Vosse. A wave propagation model of blood flow in large vessels using an approximate velocity profile function. Journal of Fluid Mechanics, 580: 145-168, 2007.

[7] B. W. A. M. M. Beulen. Toward simultaneous flow and pressure assessment in large arteries using non-invasive ultrasound. PhD thesis, Eindhoven University of Technology, 2009.

[8] E. Boileau, P. Nithiarasu, P. J. Blanco, L. O. Müller, F. E. Fossan, L. R. Hellevik, W. P. Donders, W. Huberts, M. Willemet, and J. Alastruey. A benchmark study of numerical schemes for onedimensional arterial blood flow modelling. International Journal for Numerical Methods in Biomedical Engineering, 31(10), 2015.

[9] R. F. Boucher and E. E. Kitsios. Simulation of fluid network dynamics by transmission line modelling. Proceedings of the Institution of Mechanical Engineers, Part C: Journal of Mechanical Engineering Science, 200(1):21-29, 1986.

[10] J. D. Burton, K. A. Edge, and C. R. Burrows. Modeling requirements for the parallel simulation of hydraulic systems. Journal of Dynamic Systems, Measurement, and Control, 116(1):137-145, 1994.

[11] J. E. Hall. Guyton and Hall textbook of medical physiology e-Book. Elsevier Health Sciences, 2015.

[12] J. Hashimoto, B. E. Westerhof, N. Westerhof, Y. Imai, and M. F. O’Rourke. Different role of wave reflection magnitude and timing on left ventricular mass reduction during antihypertensive treatment. Journal of Hypertension, 26(5):1017-24, 2008.

[13] K. Hayashi, H. Handa, S. Nagasawa, A. Okumura, and K. Moritake. Stiffness and elastic behavior of human intracranial and extracranial arteries. Journal of Biomechanics, 13(2):175181-179184, 1980.

[14] W. Huberts, A. S. Bode, W. Kroon, R. N. Planken, J. H. M. Tordoir, F. N. van de Vosse, and E. M. H. Bosboom. A pulse wave propagation model to support decision-making in vascular access planning in the clinic. Medical Engineering \& Physics, 34(2):233-248, 2012. 
[15] A. D. Hughes, C. Park, J. Davies, D. Francis, S. A. M. Thom, J. Mayet, and K. H. Parker. Limitations of augmentation index in the assessment of wave reflection in normotensive healthy individuals. PLOS ONE, 8(3):e59371, 2013.

[16] T. J. R. Hughes and J. Lubliner. On the one-dimensional theory of blood flow in the larger vessels. Mathematical Biosciences, 18(1-2):161-170, 1973.

[17] J. D. Humphrey. Mechanisms of arterial remodeling in hypertension: coupled roles of wall shear and intramural stress. Hypertension, 52(2):195-200, 2008.

[18] L. R. John. Forward electrical transmission line model of the human arterial system. Medical and Biological Engineering and Computing, 42(3):312-321, 2004.

[19] R. C. P. Kerckhoffs, M. L. Neal, Q. Gu, J. B. Bassingthwaighte, J. H. Omens, and A. D. McCulloch. Coupling of a 3D finite element model of cardiac ventricular mechanics to lumped systems models of the systemic and pulmonic circulation. Annals of Biomedical Engineering, 35(1):1-18, 2007.

[20] I. Korade, Z. Virag, and S. Krizmanić. A fast method for solving a linear model of one-dimensional blood flow in a viscoelastic arterial tree. Proceedings of the Institution of Mechanical Engineers, Part H: Journal of Engineering in Medicine, 231(3):203-212, 2017.

[21] W. Kroon, W. Huberts, E. M. H. Bosboom, and F. N. van de Vosse. A numerical method of reduced complexity for simulating vascular hemodynamics using coupled 0D lumped and 1D wave propagation models. Computational and Mathematical Methods in Medicine, (156094), 2012.

[22] P. Krus, K. Weddfelt, and J.-0. Palmberg. Fast pipeline models for simulation of hydraulic systems. Journal of Dynamic Systems, Measurement, and Control, 116(1):132-136, 1994.

[23] N. H. L. Kuijpers, E. Hermeling, J. Lumens, H. M. M. ten Eikelder, T. Delhaas, and F. W. Prinzen. Mechano-electrical coupling as framework for understanding functional remodeling during LBBB and CRT. American Journal of Physiology-Heart and Circulatory Physiology, 306(12): H1644-H1659, 2014.

[24] Y. Li, H. Gu, H. Fok, J. Alastruey, and P. Chowienczyk. Forward and backward pressure waveform morphology in hypertension. Hypertension, pages HYPERTENSIONAHA-116, 2016.

[25] J. Lumens, T. Delhaas, B. Kirn, and T. Arts. Three-wall segment (TriSeg) model describing mechanics and hemodynamics of ventricular interaction. Annals of Biomedical Engineering, 37 (11):2234-2255, 2009.

[26] J. Lumens, S. Ploux, M. Strik, J. Gorcsan, H. Cochet, N. Derval, M. Strom, C. Ramanathan, P. Ritter, M. Haïssaguerre, et al. Comparative electromechanical and hemodynamic effects of left ventricular and biventricular pacing in dyssynchronous heart failure: electrical resynchronization versus left-right ventricular interaction. Journal of the American College of Cardiology, 62(25): 2395-2403, 2013.

[27] R. E. Matick. Transmission lines for digital and communication networks: an introduction to transmission lines high-frequency and high-speed pulse characteristics and applications. IEEE press, 1995.

[28] F. U. S. Mattace-Raso, A. Hofman, G. C. Verwoert, J. Witteman, I. Wilkinson, J. Cockcroft, C. McEniery, S. Laurent, P. Boutouyrie, E. Bozec, et al. Determinants of pulse wave velocity in healthy people and in the presence of cardiovascular risk factors: establishing normal and reference values. European Heart Journal, 31(19):2338-2350, 2010.

[29] J. R. Mitchell and J.-J. Wang. Expanding application of the wiggers diagram to teach cardiovascular physiology. Advances in Physiology Education, 38(2):170-175, 2014. 
[30] L. O. Müller and E. F. Toro. A global multiscale mathematical model for the human circulation with emphasis on the venous system. International Journal for Numerical Methods in Biomedical Engineering, 30(7):681-725, 2014.

[31] W. Nichols, M. O'Rourke, and C. Vlachopoulos. McDonald's blood flow in arteries: theoretical, experimental and clinical principles. CRC press, 2011.

[32] M. F. O’Rourke, A. Pauca, and X.-J. Jiang. Pulse wave analysis. British Journal of Clinical Pharmacology, 51(6):507-522, 2001.

[33] G. Palau-Caballero. In silico mechanistic assessment of imaging-based measures of cardiac (patho) physiology. PhD thesis, Maastricht University, Netherlands, 2017.

[34] G. Palau-Caballero, J. Walmsley, J. Gorcsan, J. Lumens, and T. Delhaas. Abnormal ventricular and aortic wall properties can cause inconsistencies in grading aortic regurgitation severity: A computer simulation study. Journal of the American Society of Echocardiography, 29(11): 1122-1130, 2016.

[35] K. H. Parker. An introduction to wave intensity analysis. Medical and Biological Engineering and Computing, 47(2):175-88, 2009.

[36] K. H. Parker and C. J. Jones. Forward and backward running waves in the arteries: analysis using the method of characteristics. Journal of Biomechanical Engineering, 112(3):322-6, 1990.

[37] M. A. Quail, R. Short, B. Pandya, J. A. Steeden, A. Khushnood, A. M. Taylor, P. Segers, and V. Muthurangu. Abnormal wave reflections and left ventricular hypertrophy late after coarctation of the aorta repair. Hypertension, 69:501-509, 2017.

[38] J. W. Remington and E. H. Wood. Formation of peripheral pulse contour in man. Journal of Applied Physiology, 9(3):433-442, 1956.

[39] P. Reymond, F. Merenda, F. Perren, D. Rüfenacht, and N. Stergiopulos. Validation of a onedimensional model of the systemic arterial tree. American Journal of Physiology-Heart and Circulatory Physiology, 297(1):H208-H222, 2009.

[40] P. Reymond, P. Crosetto, S. Deparis, A. Quarteroni, and N. Stergiopulos. Physiological simulation of blood flow in the aorta: comparison of hemodynamic indices as predicted by 3-D FSI, 3-D rigid wall and 1-D models. Medical Engineering and Physics, 35(6):784-791, 2013.

[41] P. Segers, N. Stergiopulos, P. Verdonck, and R. Verhoeven. Assessment of distributed arterial network models. Medical and Biological Engineering and Computing, 35(6):729-736, 1997.

[42] F. N. van de Vosse and N. Stergiopulos. Pulse wave propagation in the arterial tree. Annual Review of Fluid Mechanics, 43(1):467-499, 2011.

[43] J. Walmsley, T. Arts, N. Derval, P. Bordachar, H. Cochet, S. Ploux, F. W. Prinzen, T. Delhaas, and J. Lumens. Fast simulation of mechanical heterogeneity in the electrically asynchronous heart using the multipatch module. PLOS Computational Biology, 11(7):e1004284, 2015.

[44] J. J. Wang and K. H. Parker. Wave propagation in a model of the arterial circulation. Journal of biomechanics, 37(4):457-470, 2004.

[45] T. Weber, S. Wassertheurer, M. Rammer, A. Haiden, B. Hametner, and B. Eber. Wave reflections, assessed with a novel method for pulse wave separation, are associated with end-organ damage and clinical outcomes. Hypertension, 60(2):534-41, 2012.

[46] K. H. Wesseling, J. R. Jansen, J. J. Settels, and J. J. Schreuder. Computation of aortic flow from pressure in humans using a nonlinear, three-element model. Journal of Applied Physiology, 74 (5):2566-2573, 1993. 
[47] N. Westerhof, F. Bosman, C. J. De Vries, and A. Noordergraaf. Analog studies of the human systemic arterial tree. Journal of Biomechanics, 2(2):121-143, 1969.

[48] N. Westerhof, N. Stergiopulos, M. I. Noble, and B. E. Westerhof. Wave intensity analysis. In Snapshots of Hemodynamics, pages 185-193. Springer, 2019.

[49] B. Williams, G. Mancia, W. Spiering, E. Agabiti Rosei, M. Azizi, M. Burnier, D. L. Clement, A. Coca, G. de Simone, A. Dominiczak, et al. 2018 ESC/ESH guidelines for the management of arterial hypertension. European Heart Journal, 39(33):3021-3104, 2018. 


\section{Augmentation index is not a proxy for wave reflection magnitude: Mechanistic analysis using a computational model}

The contents of this chapter are based on:

Maarten H.G. Heusinkveld, ${ }^{1}$ Tammo Delhaas, ${ }^{1}$ Joost Lumens, ${ }^{1}$ Wouter Huberts, ${ }^{1,2}$ Bart Spronck, ${ }^{3}$ Alun D. Hughes, ${ }^{4}$ Koen D. Reesink ${ }^{1}$

Augmentation index is not a proxy for wave reflection magnitude: Mechanistic analysis using a computational model. Journal of Applied Physiology (2019) 127(2):491-500

\footnotetext{
${ }^{1}$ Department of Biomedical Engineering, CARIM School for Cardiovascular Diseases, Maastricht University, Maastricht, The Netherlands.

${ }^{2}$ Department of Biomedical Engineering, Eindhoven University of Technology, Eindhoven, The Netherlands.

${ }^{3}$ Department of Biomedical Engineering, Yale University, New Haven, United States.

${ }^{4}$ Institute of Cardiovascular Sciences, University College London, London, United Kingdom.
} 


\subsection{Abstract}

The augmentation index (AIx) is deemed to capture the deleterious effect on left ventricular (LV) work of increased wave reflection associated with stiffer arteries. However, its validity as a proxy for wave reflection magnitude has been questioned. We hypothesised that, in addition to increased wave reflection due to increased pulse wave velocity, LV myocardial shortening velocity influences AIx.

Using a computational model of the circulation, we investigated the isolated and combined influences of myocardial shortening velocity $v_{\mathrm{S}, \mathrm{LV}}$ and arterial stiffness on AIx. Aortic blood pressure waveforms were characterised using AIx and the reflected wave pressure amplitude ( $\hat{p}_{\mathrm{bw}}$, obtained using wave separation analysis).

Our reference simulation (normal $v_{\mathrm{s}, \mathrm{LV}}$ and arterial stiffness) was characterised by an AIx of 21\%. A realistic reduction in $v_{\mathrm{s}, \mathrm{LV}}$ caused AIx to increase from 21 to $42 \%$. An arterial stiffness increase, characterised by a relevant $1.0 \mathrm{~m} \mathrm{~s}^{-1}$ increase in carotid-femoral pulse wave velocity, caused AIx to increase from 21 to $41 \%$. Combining the reduced $v_{\mathrm{s}, \mathrm{LV}}$ and increased arterial stiffness resulted in an AIx of $54 \%$. In a multi-step parametric analysis, both $v_{\mathrm{s}, \mathrm{LV}}$ and arterial stiffness were about equal determinants of AIx, whereas $\hat{p}_{\mathrm{bw}}$ was only determined by arterial stiffness. Furthermore, the relation between increased AIx and LV stroke work was only for about $50 \%$ explained by an increase in arterial stiffness, the other factor being $v_{\mathrm{s}, \mathrm{LV}}$. The $\hat{p}_{\mathrm{bw}}$, on the other hand, related less ambiguously to LV stroke work.

We conclude that the AIx reflects both cardiac and vascular properties and should not be considered an exclusively vascular parameter. 


\subsection{Introduction}

HE augmentation index (AIx) is defined as the late systolic boost in the aortic pressure waveform divided by pulse pressure, and is often expressed as a percentage [27] (Fig. 4.1). Pressure waveform augmentation is commonly assumed to result from the superposition of a reflected wave originating from a (discrete) reflection site in the periphery onto the incoming pressure wave generated by the heart [27]. With increased arterial stiffness, the reflected wave will propagate with increased velocity, causing the augmentation to occur earlier in systole with a consequent increase in AIx [27]. AIx is considered a vascular parameter intended to quantify the deleterious effect of systolic wave reflection on cardiac workload, which is associated with adverse cardiovascular outcomes [12].

AORTIC PRESSURE

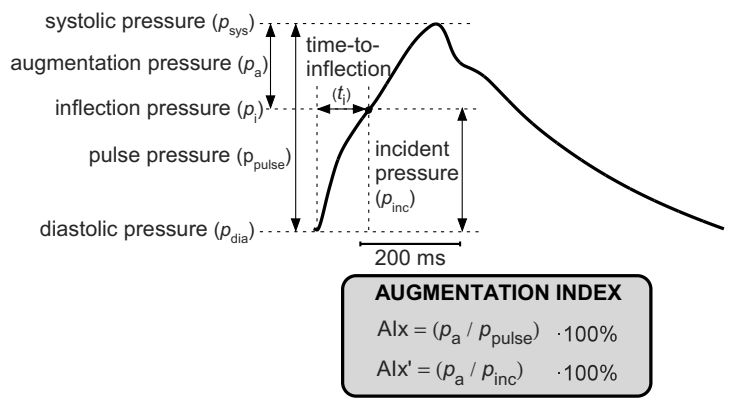

AORTIC FLOW VELOCITY

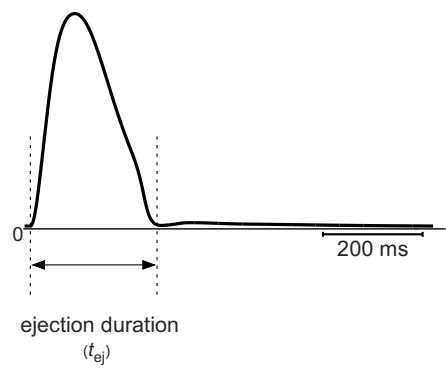

Figure 4.1: Overview of characteristics and indices extracted from the simulated aortic pressure waveform (left pane) and aortic flow waveform (right pane). Left: Augmentation index (AIx), calculated as a percentage-fraction using augmentation pressure $\left(p_{\mathrm{a}}\right)$ and pulse pressure $\left(p_{\text {pulse }}\right)$. AIx' was calculated as the percentageratio using augmentation pressure $\left(p_{\mathrm{a}}\right)$ and incident pressure $\left(p_{\text {inc }}\right)$. Time-toinflection $\left(t_{\mathrm{i}}\right)$ is defined as the time interval between foot and the inflection point at inflection pressure $p_{\mathrm{i}}$. Right pane: Left ventricular ejection duration $\left(t_{\mathrm{ej}}\right)$, the time interval during which blood is ejected into the aorta.

An advantage of AIx is its non-dimensionality requiring neither calibration of blood pressure nor measurement of blood flow velocity [19]. Blood pressure waveforms can be obtained using noninvasive tonometry at the location of the carotid- or radial arteries, or by oscillometric blood pressure recordings at brachial level $[18,20]$. Subsequently, AIx can be estimated from a synthesised central pressure waveform derived from a reconstruction algorithm, applied to the tonometric or oscillometric waveforms. The accuracy of noninvasive AIx estimation by tonometry and oscillometry was evaluated by Chen et al. [8] and Horvath et al. [18], finding a good correlation between catheter- and noninvasive AIx. Furthermore, Wilkinson et al. [45] and Savage et al. [34] reported good inter-observer reproducibility of AIx as measured by tonometry. 
The comprehensive meta-analysis of Baksi et al. [5] described AIx to drastically increase with age, despite only a small advance in time of arrival of the reflected wave. Considering the often used paradigm in clinical studies that AIx is a proxy for wave reflection magnitude $[12,19]$, one would expect similar proportions in the correlation between on the one hand age, and on the other hand AIx and wave arrival time, respectively.

Mechanistically, during systole the blood pressure waveform is the result of the instantaneous interaction between heart and arterial system. This is illustrated by the fact that aortic and left ventricular pressure patterns are in phase and closely related during $\mathrm{LV}$ ejection, when assuming normal aortic valve function [9, 43] Moreover, acute changes in arterial load are directly reflected in the time-course of ventricular and aortic pressures [21]. However, the potential influence of cardiac contractile properties on the AIx is rarely considered in clinical-epidemiological studies. Though a few clinical studies reported an association between systolic augmentation and LV diastolic dysfunction [41], the direct linkage between AIx and cardiac contractile function is difficult to determine, since AIx is likely to be confounded by other factors such as heart rate, body height and mean arterial pressure [12, 35, 37]. To overcome this issue, physics and physiology-based models of the cardiovascular system may be employed to evaluate the effect of isolated changes of cardiac- and vascular properties on AIx.

The purpose of the present study is to assess the effect of changes in LV contractility and arterial stiffness on AIx utilising the CircAdapt computational model of the circulation (www. circadapt.org) [22,40]. This closed-loop model was chosen because of its realistic cardiac mechanics model, with sarcomere length coupled to myofibre stress. Furthermore, it contains a vascular model simulating arterial and venous pressure and flow wave haemodynamics. We 1) modulated $\mathrm{LV}$ contractile properties by varying sarcomere shortening velocity $\left(v_{\mathrm{s}, \mathrm{LV}}\right)$ in the model, and 2) modulated arterial stiffness by varying the vessel stiffness parameter $(k)$. Both $v_{s, \mathrm{LV}}$ and $k$ are physiologically relevant parameters, since decreases in $v_{s, L V}$ and increases in $k$ have been associated with ageing $[1,14,16]$. We compared AIx to alternative indices of wave reflection derived using established wave separation (WS) analysis [31,32]. Finally, we evaluated the relation between AIx and cardiac workload with respect to variations in LV shortening velocity and arterial stiffness.

\subsection{Methods}

\subsubsection{The CircAdapt model}

We used the CircAdapt computational model, a closed-loop model of the fourchambered heart and circulation, to simulate cardiac mechanics and haemodynamics [22, 40].

CircAdapt consists of a limited number of modules representing cardiac walls, valves, arteries and veins as well as the systemic and pulmonary vascular beds. 
The modules directly relevant for the present study are highlighted in Fig. 4.2. Briefly, CircAdapt's contraction model constitutes of a modified Hill model, de-

CARDIAC

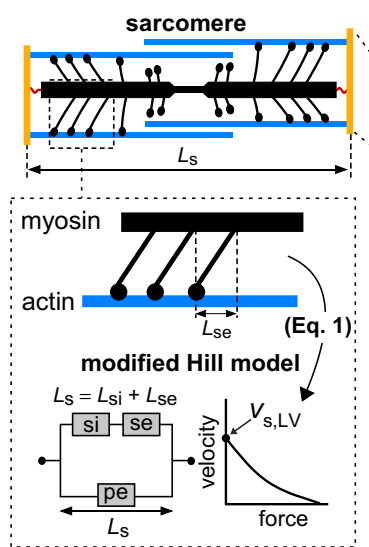

VASCULAR

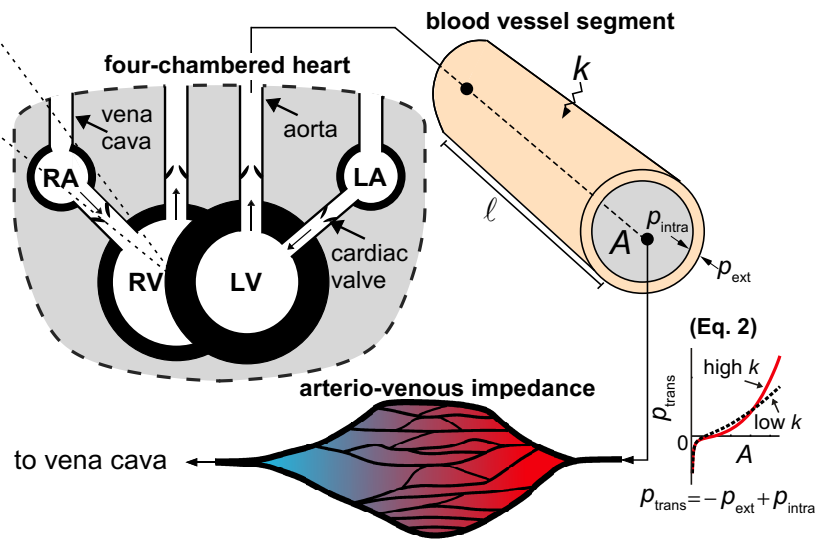

Figure 4.2: Overview of the primary relevant modules of the CircAdapt model. We distinguish two modules; the module underlying mechanics of myocardial tissue (CARDIAC) and the module underlying wave propagation in the vascular tree (VASCULAR). The sarcomere model consists of a contractile (si) element with length $L_{\mathrm{si}}$ and a series-elastic (se) element with length $L_{\mathrm{se}}$, in parallel with parallel-elastic element (pe) with sarcomere length $L_{\mathrm{s}}$ according to the model of Hill, which assumes a force-velocity relation as shown. Blood vessels are characterised by a stiffness coefficient $(k)$, intraluminal-, external- and transmural pressure ( $p_{\text {intra }}, p_{\text {ext }}$ and $p_{\text {trans }}$, respectively) and cross-sectional area $(A)$. A vessel segment with a given length $(l)$ connects a proximal node to a distal node. Peripheral vascular beds are modeled using an arterio-venous impedance model. Abbreviations: RA: right atrium, RV: right ventricle, LA: left atrium, LV: left ventricle. The model simulation code can be downloaded at https://github.com/Mheu1991/.

scribing the sarcomere mechanics (Fig. 4.2). The sarcomere model contains a contractile element with length $L_{\mathrm{si}}$, arranged in series to a series-elastic element with length $L_{\mathrm{se}}$, which in term are arranged parallel to a passive-elastic element with length $L_{\mathrm{s}}$ (Fig. 4.2). The contractile and series-elastic elements describe the additional stress generation due to muscle activation, whereas the passive-elastic element describes passive muscle behaviour (Fig. 4.2). Moreover, the length of the series-elastic element can be interpreted as the elastic deformation of the myosin heads in response to load [22]. Total sarcomere length is defined as the sum of the length of the contractile element and series element, i.e. $L_{\mathrm{s}}=L_{\mathrm{si}}+L_{\mathrm{se}}$. The rate of shortening of the contractile element $\left(\mathrm{d} L_{\mathrm{si}} / \mathrm{d} t\right)$ is given by

$$
\frac{\mathrm{d} L_{\mathrm{si}}}{\mathrm{d} t}=v_{\mathrm{s}, \mathrm{LV}}\left[\left(L_{\mathrm{se}}-L_{\mathrm{se}, \text { iso }}\right) / L_{\mathrm{se}, \text { iso }}\right] \text {, }
$$


with $v_{\mathrm{s}, \mathrm{LV}} \mathrm{LV}$ sarcomere shortening velocity, assumed $7 \mu \mathrm{m} \mathrm{s}^{-1}$ [13] and $L_{\mathrm{se} \text {,iso }}$ the length of the series elastic element at the onset of isovolumetric contraction [22]. In the model, $v_{\mathrm{s}, \mathrm{LV}}$ scales $\mathrm{d} L_{\mathrm{si}} / \mathrm{d} t$. A phenomenological mechanical activation parameter $C$ is calculated as a function of $L_{\mathrm{s}}, L_{\mathrm{si}}$ and time [22, 40]. Actively generated myofibre stress is determined using the mechanical activation, multiplied by the sarcomere extension from reference (i.e. $\left.C\left(L_{\mathrm{si}}-L_{\text {si,ref }}\right)\right)$ [40]. To obtain cavity pressures and volumes, CircAdapt uses the one-fibre model of Arts et al. [2] that allows for separation of the contribution of cardiac geometry and myofibre stress to cardiac cavity pressure, assuming myofibre stress to be homogeneously distributed within the myocardial wall. Furthermore, the heart is enclosed by the pericardium, modelled as a compliant sac [29] (Fig. 4.2).

CircAdapt enables simulation of pressure and flow waves in arteries and veins. We implemented an arterial and venous tree describing the aorta and vena cava, as well as the subclavian-, carotid-, brachial-, and femoral arteries and veins, respectively. Transmural pressure $\left(p_{\text {trans }}\right)$ and cross-sectional lumen area $(A)$ are related non-linearly, expressed by the constitutive law based on Arts et al. [4]

$$
p_{\text {trans }}(A)=-p_{\text {ext }}+p_{0}\left((1+b)\left(\frac{A}{A_{0}}\right)^{1+\frac{k / 3-2}{1+b}}-\frac{b A_{0}}{A}\right),
$$

with $p_{0}$ a reference pressure, $A_{0}$ a reference cross-sectional area, and $k$ represents the vessel stiffness coefficient, governing the non-linearity of the pressure-area relation, and thereby material stiffness (Fig. 4.2) [4]. Additionally, parameter $b$ was incorporated to avoid collapse of the tube with negative transmural pressure. Furthermore, $p_{\text {ext }}$ represents a prescribed external pressure. For our reference simulation we chose $k$-values of 8 for the aorta and vena cava. For both the arteries and veins we chose $k$-values of 14 for the subclavian, 16 for the carotid, 18 for the brachial, and 20 for the femoral, describing elastic taper (i.e. the increase in vessel wall stiffness towards the periphery [44]). Furthermore, estimations for $A_{0}$ and vessel length were based on human data given in Westerhof et al. [44]. Peripheral vascular beds were modeled using an arterio-venous impedance model [3].

\subsubsection{Simulation protocols}

Departing from our reference (REF) situation as detailed above, we simulated three additional scenarios:

- Reduced shortening velocity $\left(v_{\mathrm{s}, \mathrm{LV}}\right)$ of $\mathrm{LV}$ sarcomeres from $7 \mu \mathrm{m} \mathrm{s}^{-1}$ to 3 $\mu \mathrm{m} \mathrm{s}^{-1}$ (reduced shortening velocity; $v_{\mathrm{s}, \mathrm{LV}} \downarrow$, Table 4.1 ). To characterise the imposed change in $v_{\mathrm{s}, \mathrm{LV}}$, we calculated peak systolic strain rate, because peak systolic strain rate is considered a strong measure of LV contractility [15].

- Increased stiffness of arteries by increasing stiffness parameter $k$ (arterial stiffening; $k \uparrow$, Table 4.1). Since the modelled arterial tree contains segments 
with different $k$-values, $k$-values of all segments were increased by addition of a factor $\Delta k$. Here, $\Delta k$ was set equal to 12 (i.e. $k_{\text {stiffening }}=k_{\mathrm{REF}}+12$ ). According to clinical standards, we quantified arterial stiffness using pulse wave velocity. We obtained pulse wave velocity from transit time and travel distance. Transit time was calculated by identification of the foots of the carotid and femoral pressure waveforms. To identify the foot of the pressure wave, we used the maximum of the $2^{\text {nd }}$-order derivative [10]. Travel distance, on the other hand, was calculated as the path difference between the combined length of the aortic to femoral artery segments and aortic to carotid artery segments.

- The combined effect of a change in cardiac and vascular tissue properties (combined; $k \uparrow, v_{\mathrm{s}, \mathrm{LV}} \downarrow$, Table 4.1).

Additionally, to evaluate the dependence of AIx on LV shortening velocity and arterial stiffness, we also performed parameter sweeps of $v_{\mathrm{s}, \mathrm{LV}}$ and $\Delta k$, varying $\mathrm{LV}$ shortening velocity $v_{\mathrm{s}, \mathrm{LV}}$ from 2 to $10 \mu \mathrm{m} \mathrm{s}^{-1}$ with $1 \mu \mathrm{m} \mathrm{s}^{-1}$ increments and $\Delta k$ from -2 to 14 with increments of 1 . All simulations were performed in MATLAB 2015a (The Mathworks, Natick, USA). In all simulations, mean arterial pressure and cardiac output were maintained at $92 \mathrm{mmHg}$ and $5.11 \mathrm{~min}^{-1}$, respectively, through changes of systemic vascular resistance and circulating blood volume, while heart rate was kept constant at 72 beats $\min ^{-1}$. Collapsible tube fraction $b$ was kept at 0.02 . Haemodynamics reached steady state prior to analyses. The underlying simulation code and analysis scripts are available under the GNU General Public License v3.0 and can be downloaded from GitHub (https://github.com/Mheu1991).

\subsubsection{Wave reflection indices extracted from simulations}

Wave reflection indices were computed for simulated pressure and flow velocity waveforms of the aorta. To benchmark the relationships between simulated cardiovascular alterations and AIx, we included wave reflection indices obtained using wave separation (WS) analysis ([31], detailed below). While WS analysis yields a wave reflection index expressed as a percentage-ratio, AIx expresses wave reflection as a percentage-fraction using the expression in Fig. 4.1 [12]. Hence, we also introduce the parameter AIx', defined as a percentage-ratio of augmentation pressure to incident pressure (i.e. $p_{\mathrm{a}} / p_{\text {inc }} \cdot 100 \%$ ). Furthermore, we calculated time-to-inflection ( $t_{\mathrm{i}}$, Fig. 4.1, right pane), defined as the time-interval between the foot of the pressure waveform and inflection point [19]. Locations of foot and inflection were identified using the peak and zero-crossing of the $2^{\text {nd }}$-order derivative of the pressure curve. We extracted LV ejection duration to evaluate whether it was influenced by our simulated changes in LV shortening velocity and arterial stiffness. Using the $2^{\text {nd }}$-order derivative of the aortic valve flow velocity, LV ejection duration was calculated as the time-difference between the beginningand end of ejection (Fig. 4.1, right pane, vertical dashed lines).

The concept of WS analysis is based on solving the one-dimensional equations of mass conservation and momentum balance in elastic tubes [31]. In WS analysis, 
instantaneous changes in pressure and flow velocity, representing wavefronts, are calculated for the forward and backward directions using the water-hammer equations:

$$
\begin{aligned}
& \mathrm{d} p_{\mathrm{fw}}=\rho c \mathrm{~d} U_{\mathrm{fw}}, \text { and } \\
& \mathrm{d} p_{\mathrm{bw}}=-\rho c \mathrm{~d} U_{\mathrm{bw}},
\end{aligned}
$$

where $\mathrm{d} p_{\mathrm{fw}}$ and $\mathrm{d} U_{\mathrm{bw}}$ denote forward or backward wavefronts of pressure and flow velocity, respectively, whereas $\rho$ is the blood density $\left(1050 \mathrm{~kg} \mathrm{~m}^{-3}\right)$, and $c$ is the pulse wave velocity. The variables $\mathrm{d} p_{\mathrm{fw}}, \mathrm{d} p_{\mathrm{bw}}, \mathrm{d} U_{\mathrm{fw}}, \mathrm{d} U_{\mathrm{bw}}$ and $c$ were determined as described by Parker [31]. Through calculation of forward and backward pressures $p_{\mathrm{fw}}$ and $p_{\mathrm{bw}}$, obtained by integration of separated instantaneous pressure components, we derived wave reflection index $\hat{p}_{\mathrm{bw}} / \hat{p}_{\mathrm{fw}}$, defined as the percentage-ratio between backward pressure wave amplitude $\left(\hat{p}_{\mathrm{bw}}\right)$ and forward pressure wave amplitude $\left(\hat{p}_{\mathrm{fw}}\right)$.

\subsubsection{Calculation of cardiac workload}

To assess whether there is a relation between increased AIx and increased cardiac external work, we assessed cardiac work load by calculation of LV stroke work $\left(W_{\text {stroke }}\right)$. The variable $W_{\text {stroke }}$ was calculated as the numerically integrated area contained by the $\mathrm{LV}$ pressure $\left(p_{\mathrm{LV}}\right)$-volume $\left(V_{\mathrm{LV}}\right)$ relation:

$$
W_{\text {stroke }}=\oint p_{\mathrm{LV}} d V_{\mathrm{LV}} \text {. }
$$

\subsubsection{Statistical analysis}

Statistical analyses were performed using IBM SPSS Statistics for Windows, version 24 (IBM Corp., Armonk, NY, USA). Linear regression analysis was performed to examine the relationship between wave characteristics. $P<0.05$ was considered statistically significant.

\subsection{Results}

\subsubsection{Effects of reduced LV shortening velocity and increased arterial stiffness on augmentation index}

Fig. 4.3 and Table 4.1 contain an overview of pressure waveforms and derived characteristics for the four simulated scenarios. Simulating $v_{\mathrm{s}, \mathrm{LV}} \downarrow$ (red solid curve) did not affect absolute pressure values (Table 4.1).

However, the inflection in the pressure waveform came earlier, (from 96 to 83 ms, Table 4.1) after the foot of the pressure waveform with a marked increase 

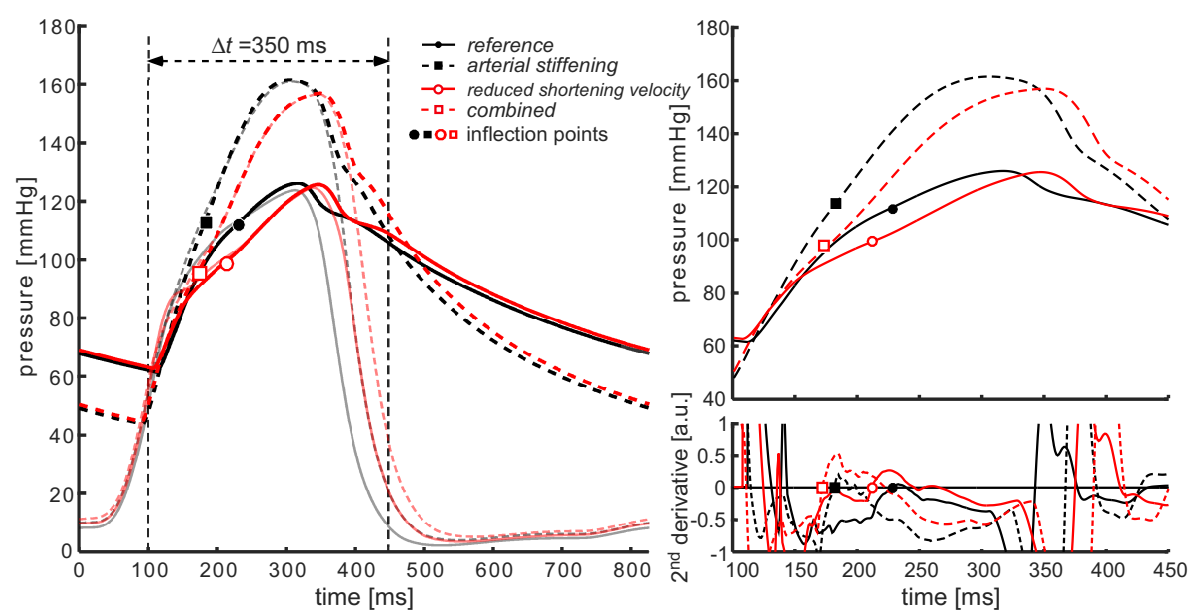

Figure 4.3: Aortic and left ventricular pressure curves for the four simulated scenarios. Left pane: Overview of simulated left ventricular and aortic pressure waveforms for four simulated scenarios. Right pane: Closer look at the systolic segment ( $t=100-450 \mathrm{~ms}$ ) of the pressure waveforms (top) and their second order derivatives (bottom). Right pane: Inflection points (black and red symbols, respectively) are located by determining the zero-crossing of the second order derivative of each simulated waveform.

in AIx (from 21 to 42\%, Table 4.1). LV ejection duration was prolonged with respect to the reference situation (from 238 to $274 \mathrm{~ms}$, Table 4.1), and peak systolic strain rate (PSSR) magnitude decreased (from $-0.91 \mathrm{~s}^{-1}$ to $-0.69 \mathrm{~s}^{-1}$, Table 4.1). Simulating $k \uparrow$ (Fig. 4.3, dashed black line), increased systolic pressure (127 to $162 \mathrm{mmHg}$ ) and decreased diastolic pressure (60 to $42 \mathrm{mmHg}$, Table 4.1). The inflection occurred significantly earlier during systole (time-to-inflection decreased from 96 to $69 \mathrm{~ms}$ ) and AIx was markedly increased (21 to 41\%, Table 4.1). The PSSR magnitude was only moderately decreased from $-0.91 \mathrm{~s}^{-1}$ to -0.80 $\mathrm{s}^{-1}$ (Table 4.1). The combined effect of $k \uparrow$ and $v_{\mathrm{s}, \mathrm{LV}} \downarrow$ resulted in a waveform that can be considered an intermediate (Fig. 4.3, red dashed curve) curve of the $k \uparrow$ and $v_{\text {s,LV }} \downarrow$ curves, with an AIx increase from 21 to 54\%. Ejection duration increased to $309 \mathrm{~ms}$ and the inflection point occurred $60 \mathrm{~ms}$ after the foot of the pressure waveform. Simulating $k \uparrow$ and $v_{\mathrm{s}, \mathrm{LV}} \downarrow$ invoked the largest reduction in PSSR magnitude, i.e. reducing from $-0.91 \mathrm{~s}^{-1}$ to $-0.64 \mathrm{~s}^{-1}$ (Table 4.1).

\subsubsection{Effects on alternative measures of wave reflection}

Fig. 4.4 contains a breakdown of simulated pressure waveforms into forward $\left(p_{\mathrm{fw}}\right)$ and backward $\left(p_{\mathrm{bw}}\right)$ pressure wave components, using wave separation (WS) analysis. Furthermore, separated pressure wave amplitudes, $\hat{p}_{\mathrm{fw}}$ and $\hat{p}_{\mathrm{bw}}$ are indicated for the REF (Fig. 4.4, left pane), $v_{\mathrm{s}, \mathrm{LV}} \downarrow$ (Fig. 4.4, centre pane) and 
Table 4.1: Influence of LV shortening velocity and arterial stiffness on blood pressure indices and reflection indices for four distinct simulated scenarios.

\begin{tabular}{cccccc}
\hline $\begin{array}{c}\text { Simulation } \rightarrow \\
\text { Metric } \downarrow\end{array}$ & $\begin{array}{c}\text { Reference } \\
(\mathrm{REF})\end{array}$ & $\begin{array}{c}\text { Reduced shortening velocity } \\
\left(v_{\mathrm{s}, \mathrm{LV}} \downarrow\right)\end{array}$ & $\begin{array}{c}\text { Arterial stiffening } \\
(k \uparrow)\end{array}$ & $\begin{array}{c}\text { Combined } \\
\left(v_{\mathrm{s}, \mathrm{LV}} \downarrow, k \uparrow\right)\end{array}$ & Unit \\
\hline$p_{\text {sys }}$ & 127 & 126 & 162 & 158 & $\mathrm{mmHg}$ \\
$p_{\text {dia }}$ & 60 & 63 & 42 & 42 & $\mathrm{mmHg}$ \\
$p_{\text {pulse }}$ & 66 & 63 & 120 & 116 & $\mathrm{mmHg}$ \\
$p_{\text {mean }}$ & 92 & 92 & 92 & 92 & $\mathrm{mmHg}$ \\
\hline AIx & 21 & 42 & 41 & 54 & $\%$ \\
Alx' & 27 & 72 & 70 & 120 & $\%$ \\
\hline$\hat{p}_{\text {bw }} / \hat{p}_{\mathrm{fw}}$ & 76 & 74 & 76 & 76 & $\%$ \\
$\hat{p}_{\text {bw }}$ & 28 & 26 & 51 & 48 & $\mathrm{mmg}$ \\
\hline time-to-inflection & 96 & 83 & 69 & 60 & $\mathrm{~ms}$ \\
ejection duration & 238 & 274 & 1.18 & 309 & $\mathrm{~ms}$ \\
\hline LV stroke work & 1.04 & 0.98 & -0.80 & -0.64 & $\mathrm{~s}$ \\
\hline PSSR & -0.91 & -0.69 & & 10 & $\mathrm{~J}$ \\
\hline
\end{tabular}

The indices $p_{\text {sys }}, p_{\text {dia }}, p_{\text {pulse }}$ and $p_{\text {mean }}$ are respectively systolic, diastolic, (systolic-diastolic) and mean aortic blood pressure, and PSSR denotes peak systolic strain rate. Note that AIx and $\hat{p}_{\mathrm{bw}} / \hat{p}_{\mathrm{fw}}$ are wave reflection indices that consider the ratio of backward and forward pressure wave amplitudes, while AIx considers the fraction of augmentation pressure and pulse pressure, respectively.

$k \uparrow$ (Fig. 4.4, right pane) simulations. Reducing LV shortening ( $\left.v_{\mathrm{s}, \mathrm{LV}}\right)$ caused $p_{\mathrm{fw}}$ to change in shape but not in amplitude as compared to the reference situation. On the contrary, no clear difference was observed in the $p_{\mathrm{bw}}$ waveform. As a result, reflection index $\hat{p}_{\mathrm{bw}} / \hat{p}_{\mathrm{fw}}$ virtually remained unchanged (76 and $74 \%$ respectively, Table 4.1). Increased arterial stiffness $(k \uparrow)$ resulted in an increase in backward pressure wave amplitude ( $\hat{p}_{\mathrm{bw}}$ ) from 28 to $51 \mathrm{mmHg}$, whereas $\hat{p}_{\mathrm{bw}} / \hat{p}_{\mathrm{fw}}$ remained unchanged (76 for both REF and $k \uparrow$, Table 4.1). The latter is attributable to a proportional increase in $\hat{p}_{\mathrm{fw}}$ and $\hat{p}_{\mathrm{bw}}$ (Fig. 4.5).

\subsubsection{Parameter sweeps}

Parameter sweeps of $v_{s, \mathrm{LV}}$ and $\Delta k$ provide more detailed insight into the relationships between on the one hand the changes in cardiac and vascular properties and on the other hand the indices AIx and $\hat{p}_{\mathrm{bw}} / \hat{p}_{\mathrm{fw}}$. Combined changes in cardiac and vascular tissue properties seem to relate non-linearly to observed changes in AIx, as is apparent in the contour plot of AIx as a function of $v_{s, \mathrm{LV}}$ and $\Delta k$ (Fig. 4.5, left pane). Our simulation results indicate that AIx is monotonically increasing with both higher arterial stiffness values and lower LV sarcomere shortening velocities. For the simulations corresponding to the white regions (i.e. high $v_{s, L V}$ and low $k$-values), no inflection point was present in the systolic phase of the aortic blood pressure curve. The relation between AIx' and changes in $v_{\mathrm{s}, \mathrm{LV}}$ and $k$ was not different, albeit that absolute values, expressing pressure wave augmentation as the ratio between pressure above the inflection point on the curve to pressure below the the inflection, ranged from 30 to $140 \%$ for all simulations (Fig. 4.5, centre pane). Using wave separation, we did not find distinctive differences in 

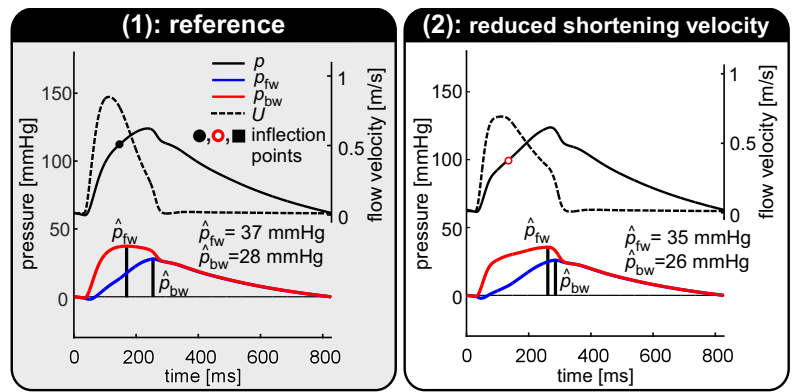

\section{(3): arterial stiffening}



Figure 4.4: Wave separation analysis performed using pressure $(p)$ and flow velocity $(U)$ waveforms obtained from 1) the reference simulation, 2) the reduced shortening velocity simulation, and 3 ) the arterial stiffening simulation. Wave reflection index $\hat{p}_{\mathrm{bw}} / \hat{p}_{\mathrm{fw}}$ was calculated as percentage-ratio of backward pressure wave amplitude $\left(\hat{p}_{\text {bw }}\right)$ and forward pressure wave amplitude $\left(\hat{p}_{\mathrm{fw}}\right)$ using wave separation (WS) analysis. The simulated aortic blood pressure waveform can be reconstructed by addition of separated pressure components ( $p_{\mathrm{fw}}$ and $\left.p_{\mathrm{bw}}\right)$. The offset is determined by the pressure at $t=0$.

the ratio between backward and forward pressure amplitude $\hat{p}_{\mathrm{bw}} / \hat{p}_{\mathrm{fw}}$ (range 72 to $76 \%$, Fig. 4.5 , right pane). This finding was caused by almost proportional increases in $\hat{p}_{\mathrm{fw}}$ and $\hat{p}_{\mathrm{bw}}$. 


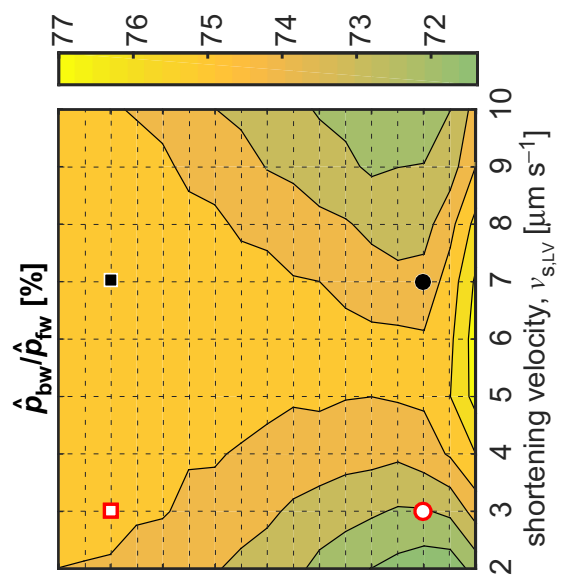

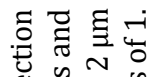

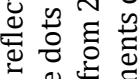
¿ 3 合䒕 过 范范管 त फै $507^{5}+$

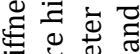
क ते है 줄

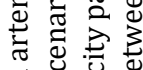
䒕

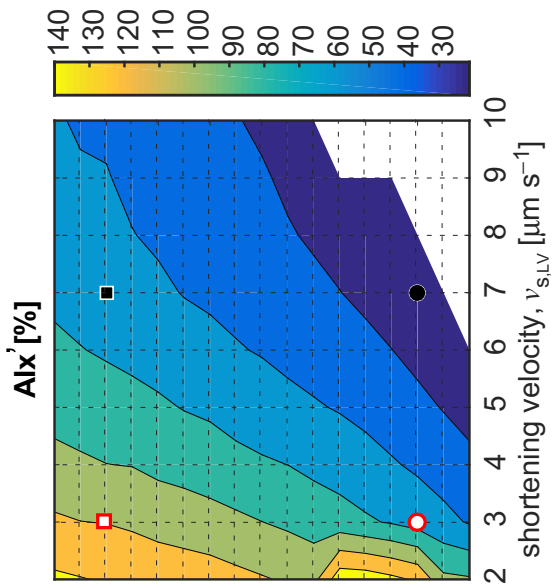
氙 ○ ये

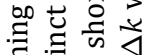
氙记 둥

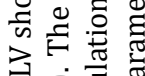
$\Xi \dot{\Phi}$ 害 का $\infty 0$ 元运可要 过这需 蛋 绐 ฮ

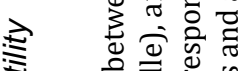

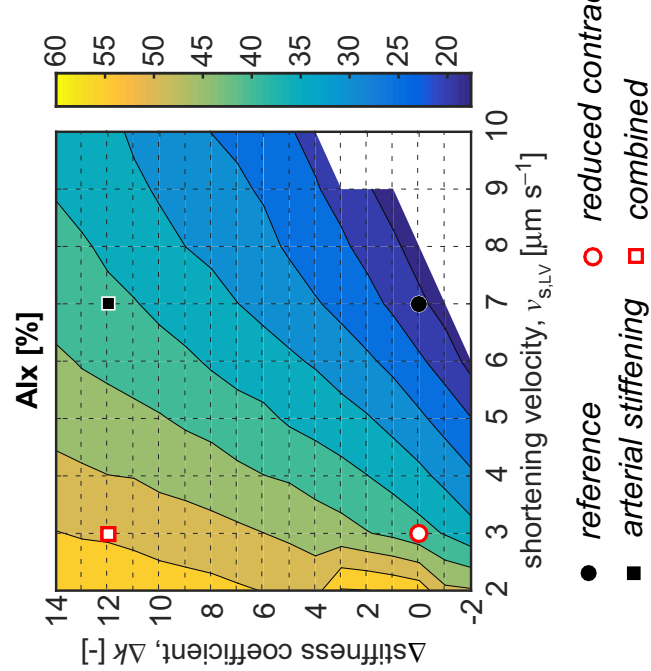
윰 ज् है 일

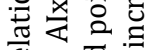

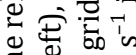

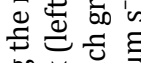
00 更

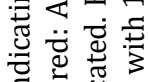
$\Xi$ 험

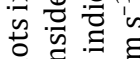
을 유ㅇㅛㅛ 웡ㅇ

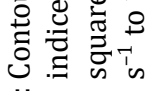

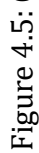



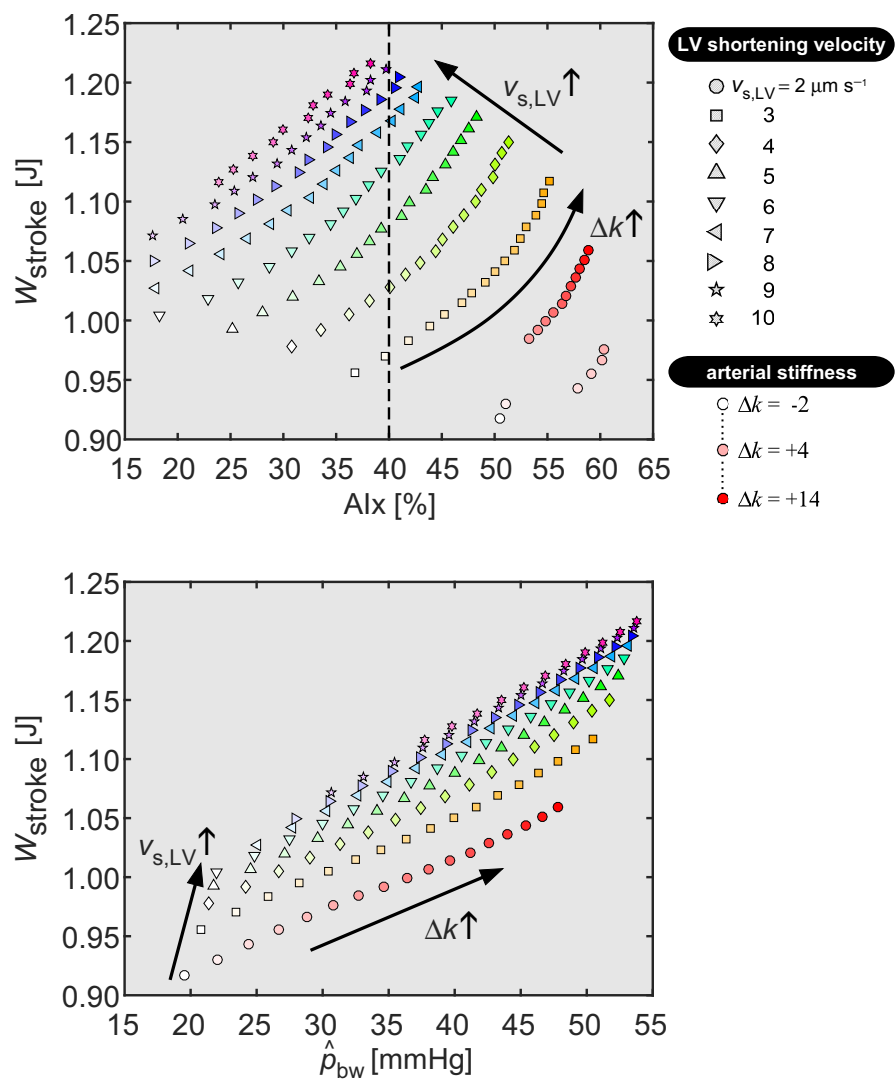

Figure 4.6: Relation between left ventricular stroke work ( $\left.W_{\text {stroke }}\right)$ and wave reflection indices, i.e. augmentation index (AIx) and backward pressure amplitude $\left(\hat{p}_{\mathrm{bw}}\right)$, respectively. Each symbol represents a simulation with a particular left ventricular sarcomere shortening velocity ( $v_{s, \mathrm{LV}}$; indicated by symbol type) and imposed arterial stiffness ( $\Delta k$; indicated using symbol colour saturation). Upper pane: Scatter plot indicating the relation between AIx and $W_{\text {stroke. The dashed line }}$ illustrates variation in $W_{\text {stroke }}$ values at an AIx value often found in elderly patients (see text). Lower pane: Scatter plot displaying the relation between $\hat{p}_{\mathrm{bw}}$ as determined using wave separation and $W_{\text {stroke. }}$. Arrows indicate the direction of changes in $v_{\mathrm{s}, \mathrm{LV}}$ and $\Delta k$ for the simulations.

\subsubsection{Relationship between Alx and left ventricular stroke work}

Fig. 4.6 (top panel) shows the relation between AIx and $W_{\text {stroke }}$ from simulations with varying arterial stiffness (indicated by colour saturation coding) and LV shortening (indicated by symbol type coding). Parameter sweeps revealed a large scatter in the relation between AIx and $W_{\text {stroke }}$ (Fig. 4.6). At a fixed AIx of $40 \%$, 
typically found in older subjects [25], $W_{\text {stroke }}$ varies by as much as $25 \%$ (Fig. 4.6, dashed line). On the other hand, scatter in the relation between $\hat{p}_{\text {bw }}$ and $W_{\text {stroke }}$ was smaller as compared to that in the relation between AIx and $W_{\text {stroke }}(\leq 11 \%$ variation in $W_{\text {stroke, }}$ for a given value of $\hat{p}_{\mathrm{bw}}$ ), suggesting an increased cardiac workload with increasing backward pressure wave amplitude (Fig. 4.6).

\subsection{Discussion}

To our knowledge, this is the first study to mechanistically study and discriminate the influence of LV contractile and arterial stiffness properties on the augmentation index. We compared our findings with alternative measures of wave reflection that were derived using wave separation analysis.

\subsubsection{Key findings and interpretation}

We found that the AIx is dependent on vascular as well as cardiac properties (Fig. 4.5). Moreover, increased AIx did not necessarily relate to increased LV workload (Fig. 4.6), which goes against the commonly used concept of increased LV workload by increased LV afterload as caused by earlier systolic wave reflections.

We explain the observed cardiac impact on AIx as follows. Given a constant stroke volume, a decreased myocardial shortening velocity will decrease LV volumetric rate $(\mathrm{LV} \mathrm{d} V / \mathrm{d} t)$ in early systole, causing a greater portion of stroke volume to be expelled during late systole. Therefore, the pressure increase prior to the inflection point will be relatively smaller than the pressure increase after the inflection point, which leads to an increased AIx. This assumes that the timing of the inflection point is primarily determined by the complex arterial impedance, which is in line with current thinking [5].

The AIx is calculated from an aortic blood pressure waveform, whereas wave separation analysis uses, in addition to blood pressure, flow (velocity) information and takes into account aortic impedance [31]. This may explain why backward pressure amplitude in our study is a better correlate of wave reflection, while AIx characterises rather the interactive influence of heart and blood vessels on the blood pressure.

Haemodynamic interaction between blood vessels and heart has been reported in patients as judged from changes in peak systolic strain rate in response to an imposed afterload change [7]. Additionally, previous experimental papers suggest that acute changes in LV myocyte external load (i.e. arterial impedance) impacts myocardial shortening $[21,33]$, corroborating that cardiac properties by themselves and/or in response to vascular changes influence AIx.

Taken together, the AIx may be an integrative marker of concurrent degenerative processes leading to increased arterial stiffness and reduced myocardial shortening velocity. Clearly, such reinterpretation of AIx requires further corroboration from clinical studies using independent cardiac and vascular measurements. As discussed in the meta-analysis of van der Waaij et al. [39], the number of clinical 
studies reporting effects of chronic changes in arterial stiffness on LV function is limited.

\subsubsection{Choice of model parameters}

Clearly, AIx will likely be determined by multiple factors, especially in humans. For our modelling study, we intentionally selected only one cardiac $\left(v_{\mathrm{s}, \mathrm{LV}}\right)$ and one vascular $(k)$ parameter with demonstrated linkages to tissue properties (myocardial shortening velocity and arterial stiffness exponent). The simulated changes in $v_{\mathrm{s}, \mathrm{LV}}$ and $k$ were by no means intended to model the clinical-epidemiological profiles of heart failure or vascular ageing.

The imposed reduction in LV shortening velocity $\left(v_{\mathrm{s}, \mathrm{LV}} \downarrow\right)$ significantly changed the peak systolic strain rate from $-0.91 \mathrm{~s}^{-1}$ for the reference simulation to $-0.69 \mathrm{~s}^{-1}$ (i.e. a reduction in magnitude of $24 \%$ ). This decrease in peak systolic strain rate is physiologically plausible as based on reference data reported in Dalen et al. [11]. In previous work, we conducted a local sensitivity analysis assessing how changes in cardiac CircAdapt model parameters affect aortic augmentation index [17]. In this analysis, testing four candidate cardiac parameters, we found $v_{\mathrm{s}, \mathrm{LV}}$ to be the most important cardiac determinant of augmentation index which motivated us to select this model parameter in our analysis. We chose to reduce $v_{\mathrm{s}, \mathrm{LV}}$ based on rat experimental data reporting force-velocity relations in papillary muscle [1]. These data showed a linear decrease in peak muscle shortening velocity of $\approx 50 \%$ with increasing age (i.e. 100 to 1000 days). Moreover, in isolated human ventricular myocytes, a reduction in shortening velocity with age was also observed [16]. The arterial stiffening simulation $(k \uparrow)$ corresponded to a relevant carotid-to-femoral pulse wave velocity increase from $6.6 \mathrm{~m} \mathrm{~s}^{-1}$ to $7.6 \mathrm{~m} \mathrm{~s}^{-1}$.

\subsubsection{Alternative metrics in relation to wave reflection magnitude}

Augmentation index expressed as a percentage-ratio (i.e. the parameter AIx') showed the same dependency on vascular and cardiac properties as was found for the regular AIx. In contrast to AIx and AIx', the ratio of backward and forward pressure wave amplitude calculated by means of wave separation analysis remained relatively unaffected for all simulations (Fig. 4.5). This was caused by a proportional increase in forward pressure wave amplitude with increased arterial stiffness. Such increased forward pressure wave amplitude with age was also observed in men and women in the Framingham Heart Study and was associated with age-related stiffening of central arteries [38]. Our simulations did show a clear pattern between arterial stiffness and backward wave amplitude $\left(\hat{p}_{\mathrm{bw}}\right)$ as determined using wave separation analysis. Moreover, the pattern was independent of LV sarcomere shortening velocity (Fig. 4.7).

Potential implications of increased backward wave amplitude with respect to cardiovascular risk were reported by Weber et al. [42]. Two key findings of their 




Figure 4.7: Effect of changes in sarcomere shortening velocity $v_{s, L V}$ and vessel stiffness $(k)$ on backward pressure amplitude $\left(\hat{p}_{\text {bw }}\right)$ assessed using wave separation (WS) analysis. The distinct simulated scenarios are highlighted by the dots and squares as indicated. Each grid point corresponds to a single simulation.

study were that 1) backward wave amplitude was the most consistent predictor of a composite cardiovascular end point, including mortality, in a group of 725 patients undergoing coronary angiography, and 2) the predictive value of AIx and augmentation pressure was inferior to that of backward wave amplitude [42] The present study supports their findings and extends the explanation towards cardiac influences.

One should take caution when interpreting our simulated scenarios as emulators of human ageing and, hence, to expect an increased reflection magnitude as has been reported by Segers et al. [36] in the ageing population. Several aspects regarding cardio-vascular structure and function (e.g. cardiac mass and vascular segment diameters) that reportedly change with age have been kept constant throughout our simulations. Previously, Maksuti et al. [24] and Pagoulatou and Stergiopulos [28] performed simulation studies focussing on changes in arterial haemodynamics during physiological ageing, whereas Willemet et al. [46] introduced a method to generate virtual cohorts consisting of arterial haemodynamics models, with varying population characteristics including age. Future studies on the evolution of AIx and other wave reflection indices may consider these methods as a take-off point.

Simulations revealed a linear association between ejection duration and augmentation index. Based on the simulations obtained in our multi-step parametric analysis, an increase in ejection duration of $20 \mathrm{~ms}$ caused AIx to increase with 9-percentage points (i.e. indicated by an unstandardised $B$ of $0.43 \% / \mathrm{ms}, 95 \%$ confidence interval $=[0.40,0.45] \% / \mathrm{ms}, P<0.001)$. In the study of Sharman et al. 
[37], a similar association between ejection duration and augmentation index of 10 percentage point per $20 \mathrm{~ms}$ of increase in ejection duration was found. In our model, this association is explained by a decrease in LV early-systolic volumetric rate with constant stroke volume, causing a significant portion of the LV stroke volume to be expelled after the inflection point (see above).

\subsubsection{Cardiac workload}

In the present study, we quantified cardiac workload by calculating left ventricular stroke work. Increasing arterial stiffness caused left ventricular stroke work to increase $(+13 \%)$. Left ventricular stroke work slightly decreased $(-6 \%)$ for the simulation with reduced left ventricular shortening velocity (Table 4.1). While AIx is derived from arterial measurements, using it to quantify cardiac workload appears to be erroneous, as cardiac contractility plays a role as well. Results imply that backward pressure amplitude — rather than depending solely on arterial stiffness in these simulations - to be more indicative of cardiac workload than AIx.

\subsubsection{Previous clinical studies on ventriculo-arterial interaction}

Below, two examples of clinical studies investigating aspects of ventriculo-arterial interaction are discussed. Bell et al. [6] reported that during systole the proximal aorta elongates, imposing a higher stretch-related workload on the LV. During diastole, the amount of elastic recoil energy is positively associated with increased early diastolic LV filling, suggesting that elastic recoil of the proximal aorta may benefit LV diastolic function through mechanical ventriculo-arterial coupling. In their analyses on the relations between aortic and LV measures, statistical correction of the (potential) confounding effect of wave reflections was performed using AIx. Given the present results, adjusting for AIx as a proxy of wave reflection may lead to over or undercorrection for the cardiac influence.

Palombo et al. [30] investigated the treatment effects of a calcium-channel blocker on LV structure (e.g. LV mass) and function (e.g. LV stroke work) indices as well as central (aortic) haemodynamics indices. Findings from the study included that the ratio of backward pressure amplitude and forward pressure amplitude was equal between hypertensive patients and normotensive controls, while backward pressure amplitude and LV stroke work were significantly higher for the hypertensive patient group. These findings corroborate our simulation findings that $\hat{p}_{\text {bw }}$ best reflects the impact of backward wave reflection with increased arterial stiffness on LV workload.

\subsubsection{Limitations}

When interpreting our results, one has to realise that the data presented were not based on patient measurements but come from computer simulations. Although 
we did not fit our model to patient data, the order of magnitude of the AIx compares well with human studies. In our reference simulation, AIx was found to be equal to $21 \%$, which is in accordance with the range of values reported by Hughes et al. [19] in 65 healthy individuals ( $44 \pm 14$ years) and by Savage et al. [34] in 188 patients ( $56 \pm 15$ years) with chronic renal failure. Imposing either reduced LV shortening velocity or increased arterial stiffness caused AIx to increase to 41 and $42 \%$, respectively. This is equivalent to the AIx measured in subjects between 70 and 80 years of age [26].

The following aspects regarding model assumptions and choices warrant discussion: The CircAdapt model used in this study describes a highly simplified cardiac geometry, relating myofibre stress and strain by single values. This approach was shown to be valid under the assumption that myofibre stress is homogeneously distributed [2]. A previous study utilising the CircAdapt model obtained physiologically realistic LV strain patterns as compared to global LV strain patterns obtained from MR-tagging [23]. Therefore, we believe CircAdapt's contraction model is appropriate for our study's purpose.

Furthermore, the wave propagation model we used neglects non-linear convective acceleration (i.e. we assume linear wave behaviour), thereby introducing small modelling error in pressure and flow waveforms. However, the implication of such error to derived haemodynamic indices is negligible in our study, since all the wave reflection indices we derived also assume linear wave behaviour.

We simulated arterial stiffening by increasing material stiffness parameter $k$ (Eq. 4.2). Mean arterial pressure (i.e. $p_{0}$ ) was assumed to remain constant. As such, systolic blood pressure increased, whereas diastolic blood pressure decreased, which may not be representative for patients for which diastolic blood pressure is increased as well, e.g. due to increased peripheral resistance. To assess the implication of keeping mean arterial pressure constant on our findings, we repeated the reference, reduced shortening velocity, arterial stiffening and combined simulations with $p_{0}$ kept at $102 \mathrm{mmHg}$ and $112 \mathrm{mmHg}$, respectively. With respect to changes $(\Delta)$ in augmentation index and backward pressure wave amplitude relative to the reference situation (i.e. the $\left[v_{s, \mathrm{LV}} \downarrow-\mathrm{REF}\right],[k \uparrow-\mathrm{REF}]$, and $\left[v_{\mathrm{S}, \mathrm{LV}} \downarrow, k \uparrow-\mathrm{REF}\right]$ differences), we found only a minor influence of increasing $p_{0}$ (i.e. $\Delta \mathrm{AIx} \leq 8 \%$ and $\Delta \hat{p}_{\text {bw }} \leq 5 \mathrm{mmHg}$, respectively).

We believe our model-based findings require further confirmation in clinical studies. Such a study could be a case-control design with isolated systolic hypertension and normotensive patient groups. Measurements in these studies should include speckle-tracking echocardiography to characterise LV contractile function [11], pulse wave analysis to characterise augmentation index [12], wave separation analysis [31] to characterise wave reflection behaviour, as well as stroke volume and blood pressure measurements to estimate cardiac workload. 


\subsection{Conclusion}

We conclude that the AIx reflects both cardiac and vascular properties, and hence should not be considered a vascular parameter. Furthermore, an increase in AIx does not necessarily relate to increased stroke work. Hence, we believe AIx should be abandoned as a proxy for increased wave reflection magnitude due to an arterial stiffness increase.

\section{Acknowledgments}

M.H.G. Heusinkveld was supported by a Kootstra Talent Fellowship from Maastricht University Medical Centre. A. Hughes and K. Reesink were supported by the British Heart Foundation (BHF; grant PG29934). J. Lumens acknowledges support from the Dr. Dekker Program of the Dutch Heart Foundation (grant 2015T082) and the Netherlands Organisation for Scientific Research (NWO-ZonMw, VIDI grant 016.176.340). 



\section{References}

[1] N. R. Alpert, L. A. Mulieri, and D. Warshaw. The failing human heart. Cardiovascular Research, 54 (1):1-10, 2002.

[2] T. Arts, P. H. M. Bovendeerd, F. W. Prinzen, and R. S. Reneman. Relation between left ventricular cavity pressure and volume and systolic fiber stress and strain in the wall. Biophysical Journal, 59(1):93-102, 1991.

[3] T. Arts, J. Lumens, W. Kroon, and T. Delhaas. Control of whole heart geometry by intramyocardial mechano-feedback: a model study. PLOS Computational Biology, 8(2):e1002369, 2012.

[4] T. Arts, K. D. Reesink, W. Kroon, and T. Delhaas. Simulation of adaptation of blood vessel geometry to flow and pressure: Implications for arterio-venous impedance. Mechanics Research Communications, 42:15-21, 2012.

[5] A. J. Baksi, T. A. Treibel, J. E. Davies, N. Hadjiloizou, R. A. Foale, K. H. Parker, D. P. Francis, J. Mayet, and A. D. Hughes. A meta-analysis of the mechanism of blood pressure change with aging. Journal of the American College of Cardiology, 54(22):2087-2092, 2009.

[6] V. Bell, S. Sigurdsson, J. J. Westenberg, J. D. Gotal, A. A. Torjesen, T. Aspelund, L. J. Launer, T. B. Harris, V. Gudnason, A. de Roos, et al. Relations between aortic stiffness and left ventricular structure and function in older participants in the age, gene/environment susceptibility-reykjavik study. Circulation: Cardiovascular Imaging, 8(4):e003039, 2015.

[7] A. T. Burns, A. La Gerche, J. D’Hooge, A. I. MacIsaac, and D. L. Prior. Left ventricular strain and strain rate: characterization of the effect of load in human subjects. European Journal of Echocardiography, 11(3):283-289, 2009.

[8] C. H. Chen, C. T. Ting, A. Nussbacher, E. Nevo, D. A. Kass, P. Pak, S. P. Wang, M. S. Chang, and F. C. Yin. Validation of carotid artery tonometry as a means of estimating augmentation index of ascending aortic pressure. Hypertension, 27(2):168-75, 1996.

[9] J. A. Chirinos and P. Segers. Noninvasive evaluation of left ventricular afterload: part 2: arterial pressure-flow and pressure-volume relations in humans. Hypertension, 56(4):563-70, 2010.

[10] Y. C. Chiu, P. W. Arand, S. G. Shroff, T. Feldman, and J. D. Carroll. Determination of pulse wave velocities with computerized algorithms. American Heart Journal, 121(5):1460-70, 1991.

[11] H. Dalen, A. Thorstensen, S. A. Aase, C. B. Ingul, H. Torp, L. J. Vatten, and A. Stoylen. Segmental and global longitudinal strain and strain rate based on echocardiography of 1266 healthy individuals: the HUNT study in Norway. European Journal of Echocardiography, 11(2):176-183, 2009.

[12] J. I. Davies and A. D. Struthers. Pulse wave analysis and pulse wave velocity: a critical review of their strengths and weaknesses. Journal of Hypertension, 21(3):463-72, 2003.

[13] P. P. de Tombe and H. E. D. J. ter Keurs. Force and velocity of sarcomere shortening in trabeculae from rat heart. effects of temperature. Circulation Research, 66(5):1239-1254, 1990.

[14] L. Engelen, I. Ferreira, C. D. A. Stehouwer, P. Boutouyrie, and S. Laurent. Reference intervals for common carotid intima-media thickness measured with echotracking: relation with risk factors. European Heart Journal, 34(30):2368-80, 2013. 
[15] N. L. Greenberg, M. S. Firstenberg, P. L. Castro, M. Main, A. Travaglini, J. A. Odabashian, J. K. Drinko, L. L. Rodriguez, J. D. Thomas, and M. J. Garcia. Doppler-derived myocardial systolic strain rate is a strong index of left ventricular contractility. Circulation, 105(1):99-105, 2002.

[16] S. E. Harding, S. M. Jones, P. O’Gara, F. del Monte, G. Vescovo, and P. A. Poole-Wilson. Isolated ventricular myocytes from failing and non-failing human heart; the relation of age and clinical status of patients to isoproterenol response. Journal of Molecular and Cellular Cardiology, 24(5): 549-64, 1992.

[17] M. H. G. Heusinkveld. Insight in heart-vessel interaction by combining computational modeling and routine clinical measurements. Master's thesis, Eindhoven University of Technology, 2015.

[18] I. G. Horvath, A. Nemeth, Z. Lenkey, N. Alessandri, F. Tufano, P. Kis, B. Gaszner, and A. Cziraki. Invasive validation of a new oscillometric device (arteriograph) for measuring augmentation index, central blood pressure and aortic pulse wave velocity. Journal of Hypertension, 28(10): 2068-2075, 2010.

[19] A. D. Hughes, C. Park, J. Davies, D. Francis, G. T. S. A. Mc, J. Mayet, and K. H. Parker. Limitations of augmentation index in the assessment of wave reflection in normotensive healthy individuals. PLOS ONE, 8(3):e59371, 2013.

[20] R. Kelly, C. Hayward, A. Avolio, and M. O’Rourke. Noninvasive determination of age-related changes in the human arterial pulse. Circulation, 80(6):1652-1659, 1989.

[21] A. F. Leite-Moreira, J. Correia-Pinto, and T. C. Gillebert. Afterload induced changes in myocardial relaxation: a mechanism for diastolic dysfunction. Cardiovascular Research, 43(2):344-353, 1999.

[22] J. Lumens, T. Delhaas, B. Kirn, and T. Arts. Three-wall segment (TriSeg) model describing mechanics and hemodynamics of ventricular interaction. Annals of Biomedical Engineering, 37 (11):2234-55, 2009.

[23] J. Lumens, T. Arts, J. T. Marcus, A. Vonk-Noordegraaf, and T. Delhaas. Early-diastolic left ventricular lengthening implies pulmonary hypertension-induced right ventricular decompensation. Cardiovascular Research, 96(2):286-295, 2012.

[24] E. Maksuti, N. Westerhof, B. E. Westerhof, M. Broomé, and N. Stergiopulos. Contribution of the arterial system and the heart to blood pressure during normal aging-a simulation study. PLOS ONE, 11(6):e0157493, 2016.

[25] C. M. McEniery, I. R. Hall, A. Qasem, I. B. Wilkinson, J. R. Cockcroft, A. Investigators, et al. Normal vascular aging: differential effects on wave reflection and aortic pulse wave velocity: the Anglo-Cardiff Collaborative Trial (ACCT). Journal of the American College of Cardiology, 46(9): 1753-1760, 2005.

[26] M. Namasivayam, B. J. McDonnell, C. M. McEniery, and M. F. O’Rourke. Does wave reflection dominate age-related change in aortic blood pressure across the human life span? Hypertension, 53(6):979-985, 2009.

[27] W. Nichols, M. O'Rourke, and C. Vlachopoulos. McDonald's blood flow in arteries: theoretical, experimental and clinical principles. CRC Press, 2011. ISBN 1444128787.

[28] S. Pagoulatou and N. Stergiopulos. Evolution of aortic pressure during normal ageing: A modelbased study. PLOS ONE, 12(7):e0182173, 2017.

[29] G. Palau-Caballero. In silico mechanistic assessment of imaging-based measures of cardiac (patho) physiology. PhD thesis, Maastricht University, Netherlands, 2017. 
[30] C. Palombo, E. Malshi, C. Morizzo, F. Rakebrandt, V. Corretti, F. Santini, A. G. Fraser, and M. Kozakova. Arterial wave reflection during antihypertensive therapy with barnidipine: a 6-month, open-label study using an integrated cardiovascular ultrasound approach in patients with newly diagnosed hypertension. Clinical Therapeutics, 31(12):2873-2885, 2009.

[31] K. H. Parker. An introduction to wave intensity analysis. Medical and Biological Engineering and Computing, 47(2):175-88, 2009.

[32] K. H. Parker and C. J. Jones. Forward and backward running waves in the arteries: analysis using the method of characteristics. Journal of Biomechanical Engineering, 112(3):322-6, 1990.

[33] J. Ross Jr. Afterload mismatch and preload reserve: a conceptual framework for the analysis of ventricular function. Progress in Cardiovascular Diseases, 18(4):255-264, 1976.

[34] M. T. Savage, C. J. Ferro, S. J. Pinder, and C. R. Tomson. Reproducibility of derived central arterial waveforms in patients with chronic renal failure. Clinical Science, 103(1):59-65, 2002.

[35] M. T. Schram, R. M. A. Henry, R. A. J. M. van Dijk, P. J. Kostense, J. M. Dekker, G. Nijpels, R. J. Heine, L. M. Bouter, N. Westerhof, and C. D. A. Stehouwer. Increased central artery stiffness in impaired glucose metabolism and type 2 diabetes: the Hoorn Study. Hypertension, 43(2):176-181, 2004.

[36] P. Segers, E. R. Rietzschel, M. L. De Buyzere, S. J. Vermeersch, D. De Bacquer, L. M. Van Bortel, G. De Backer, T. C. Gillebert, and P. R. Verdonck. Noninvasive (input) impedance, pulse wave velocity, and wave reflection in healthy middle-aged men and women. Hypertension, 49(6): 1248-1255, 2007.

[37] J. E. Sharman, J. E. Davies, C. Jenkins, and T. H. Marwick. Augmentation index, left ventricular contractility, and wave reflection. Hypertension, 54(5):1099-105, 2009.

[38] A. A. Torjesen, N. Wang, M. G. Larson, N. M. Hamburg, J. A. Vita, D. Levy, E. J. Benjamin, R. S. Vasan, and G. F. Mitchell. Forward and backward wave morphology and central pressure augmentation in men and women in the framingham heart study. Hypertension, 64(2):259-65, 2014.

[39] K. M. van der Waaij, M. H. G. Heusinkveld, T. Delhaas, A. A. Kroon, and K. D. Reesink. Do treatmentinduced changes in arterial stiffness affect left ventricular structure? A meta-analysis. Journal of Hypertension, 37(2):253-263, 2019.

[40] J. Walmsley, T. Arts, N. Derval, P. Bordachar, H. Cochet, S. Ploux, F. W. Prinzen, T. Delhaas, and J. Lumens. Fast simulation of mechanical heterogeneity in the electrically asynchronous heart using the multipatch module. PLOS Computational Biology, 11(7):e1004284, 2015.

[41] T. Weber, J. Auer, M. F. O’Rourke, C. Punzengruber, E. Kvas, and B. Eber. Prolonged mechanical systole and increased arterial wave reflections in diastolic dysfunction. Heart, 92(11):1616-22, 2006.

[42] T. Weber, S. Wassertheurer, M. Rammer, A. Haiden, B. Hametner, and B. Eber. Wave reflections, assessed with a novel method for pulse wave separation, are associated with end-organ damage and clinical outcomes. Hypertension, 60(2):534-41, 2012.

[43] N. Westerhof and M. F. O’Rourke. Haemodynamic basis for the development of left ventricular failure in systolic hypertension and for its logical therapy. Journal of Hypertension, 13(9):943-52, 1995.

[44] N. Westerhof, F. Bosman, C. J. De Vries, and A. Noordergraaf. Analog studies of the human systemic arterial tree. Journal of Biomechanics, 2(2):121-43, 1969.

[45] I. B. Wilkinson, S. A. Fuchs, I. M. Jansen, J. C. Spratt, G. D. Murray, J. R. Cockcroft, and D. J. Webb. Reproducibility of pulse wave velocity and augmentation index measured by pulse wave analysis. Journal of Hypertension, 16(12):2079-2084, 1998. 
[46] M. Willemet, P. Chowienczyk, and J. Alastruey. A database of virtual healthy subjects to assess the accuracy of foot-to-foot pulse wave velocities for estimation of aortic stiffness. American Journal of Physiology-Heart and Circulatory Physiology, 309:H663-H675, 2015. 


\section{Complementing sparse vascular imaging data by physiological adaptation rules}

The contents of this chapter are based on:

Maarten H.G. Heusinkveld, ${ }^{1}$ Robert J. Holtackers, ${ }^{2}$ Bouke P. Adriaans, ${ }^{2,3}$ Jos Op 't Roodt, ${ }^{4}$ Theo Arts, ${ }^{1}$ Tammo Delhaas, ${ }^{1}$ Koen D. Reesink, ${ }^{1}$ Wouter Huberts ${ }^{1,5}$ Complementing sparse vascular imaging data by physiological adaptation rules. (In revision with Journal of Applied Physiology)

\footnotetext{
${ }^{1}$ Department of Biomedical Engineering, CARIM School for Cardiovascular Diseases, Maastricht University, Maastricht, The Netherlands.

${ }^{2}$ Department of Radiology, CARIM School for Cardiovascular Diseases, Maastricht University, Maastricht, The Netherlands.

${ }^{3}$ Department of Cardiology, Maastricht University Medical Centre, Maastricht, The Netherlands.

${ }^{4}$ Department of Internal Medicine, Maastricht University Medical Centre, Maastricht, The Netherlands.

${ }^{5}$ Department of Biomedical Engineering, Eindhoven University of Technology, Eindhoven, The Netherlands.
} 


\subsection{Abstract}

\section{Introduction:}

Mathematical modelling of pressure and flow waveforms in blood vessels using pulse wave propagation (PWP) models has tremendous potential to support clinical decision-making. For a personalised model outcome, measurements of all modelled vessel radii and wall thicknesses are required. In clinical practice, however, data sets are often incomplete. To overcome this problem, we hypothesised that the adaptive capacity of vessels in response to mechanical load could be utilised to fill in the gaps of incomplete patient-specific data sets.

\section{Methods:}

We implemented homeostatic feedback loops in a validated PWP model to allow adaptation of vessel geometry to maintain physiological values of wall stress and wall shear stress. To evaluate our approach, we gathered vascular MRI and ultrasound data sets of wall thicknesses and radii of central and arm arterial segments of ten healthy subjects. Reference models (i.e. termed REFMoDEL, $n=10$ ) were simulated using complete data, whereas adapted models (ADAPTMODEL, $n=10$ ) used data of one carotid artery segment only while the remaining geometries of this model were estimated using adaptation. In both models, mean brachial pressure, vascular distensibility, heart rate, aortic inflow and cardiac output distribution were prescribed. We evaluated agreement between REFMoDEL and ADAPTModEL geometries, as well as between pressure and flow waveforms of both models.

\section{Results:}

Limits of agreement (bias \pm 2 SD of difference) between ADAPTMODEL and REFMoDEL radii and wall thicknesses were $0.2 \pm 2.6 \mathrm{~mm}$ and $-140 \pm 557 \mu \mathrm{m}$, respectively. A comparative analysis between ADAPTMODEL pressure and flow waveform characteristics and those generated by the REFMODEL showed adequate agreement.

\section{Conclusions:}

In conclusion, our adaptation-based PWP model enables personalisation of vascular geometries even when not all required data is available. 


\subsection{Glossary of terms}

\begin{tabular}{|c|c|c|}
\hline \multicolumn{3}{|l|}{ General } \\
\hline$\eta$ & Pa s & blood dynamic viscosity \\
\hline$\rho$ & $\mathrm{kg} \mathrm{m}^{-3}$ & blood density \\
\hline$t$ & $\mathrm{~s}$ & time \\
\hline$V_{r}$ & $\%$ & coefficient of variance for tube radius \\
\hline$V_{h}$ & $\%$ & coefficient of variance for tube wall thickness \\
\hline \multicolumn{3}{|c|}{ PWP model } \\
\hline$\alpha_{0}$ & - & characteristic Womersley number \\
\hline$\mu$ & - & Poisson's ratio \\
\hline$\delta$ & - & convection term multiplication factor \\
\hline$\zeta_{c}$ & - & fraction of lumen area with inertia dominated flow \\
\hline$A$ & $\mathrm{~m}^{2}$ & vessel lumen cross-sectional area \\
\hline$A_{\text {wall }}$ & $\mathrm{m}^{2}$ & vessel cross-sectional wall area \\
\hline$c_{\text {pulse }}$ & $\mathrm{m} \mathrm{s}^{-1}$ & carotid-to-radial pulse wave velocity \\
\hline$C^{2}$ & $\mathrm{~m}^{2} \mathrm{~Pa}^{-1}$ & area compliance \\
\hline$C_{\text {art }}$ & $\mathrm{m}^{3} \mathrm{~Pa}^{-1}$ & arterial compliance \\
\hline$D_{0}$ & $\mathrm{~Pa}^{-1}$ & distensibility \\
\hline E & $\mathrm{Pa}$ & Young's modulus \\
\hline$h$ & $\mathrm{~m}$ & vessel wall thickness \\
\hline$L$ & $\mathrm{~Pa} \mathrm{~s}^{2} \mathrm{~m}^{-4}$ & inertance per unit length \\
\hline$p$ & $\mathrm{~Pa}$ & transmural pressure \\
\hline$q$ & $\mathrm{~m}^{3} \mathrm{~s}^{-1}$ & flow rate \\
\hline$r$ & $\mathrm{~m}$ & vessel lumen radius \\
\hline$R_{\mathrm{p}}$ & Pa s m ${ }^{-3}$ & peripheral resistance \\
\hline$z^{P}$ & $\mathrm{~m}$ & axial vessel coordinate \\
\hline & Pa s m ${ }^{-3}$ & characteristic impedance \\
\hline \multicolumn{3}{|c|}{ Vascular adaptation } \\
\hline$\sigma_{\mathrm{f}, \text { target }}$ & $\mathrm{Pa}$ & peak wall stress target value \\
\hline $\bar{\tau}_{\text {target,prox }}$ & $\mathrm{Pa}$ & wall shear stress target value for proximal arteries \\
\hline $\bar{\tau}_{\text {target,distal }}$ & $\mathrm{Pa}$ & wall shear stress target value for distal arteries \\
\hline$k_{A}$ & - & wall thickness adaptation feedback factor \\
\hline$k_{r}$ & - & radius adaptation feedback factor \\
\hline$r_{\text {crit }}$ & & critical vessel radius for radius adaptation \\
\hline$v_{\text {impact }}$ & $\mathrm{m} \mathrm{s}^{-1}$ & body impact velocity \\
\hline
\end{tabular}




\subsection{Introduction}

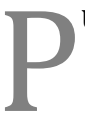

ULSE wave propagation (PWP) models, as governed by the 1D equations of momentum balance and mass conservation [15], describe propagation of pressure-flow waves within networks of (elastic) tubes. In cardiovascular research, PWP models have been applied to relate changes in blood flow characteristics (e.g. mean flow rate or systolic blood pressure [40]) to vascular pathologies, such as arterial stenosis (i.e. local narrowing of blood vessels) and aneurysms (i.e. pathological widening of blood vessels). PWP models are also used to predict the outcome of interventions in individual patients [7,13]. Personalised clinical applications of PWP models require patient-specific clinical data to parametrise the model.

However, personalisation of PWP models is usually hampered because only limited amount of clinical data is available due to time constraint, accessibility, procedural costs or loss of data. One way to deal with sparse clinical data is to perform an extensive sensitivity analysis to help prioritise the PWP-model parameters that require patient-specific measurements [14]. Another option is to reduce model complexity, reducing the number of 1D arterial branches within an arterial domain, by lumping them into less complex 0D windkessel models instead [10]. However, caveats to lumping are the loss of spatial information and the loss of physiological meaning of the resulting lumped parameters, which may not be acceptable for some applications.

In the present paper, we propose a completely different and innovative approach to complement sparse data on arterial radius and wall thickness by utilising knowledge of the adaptive capacity of the human vasculature in response to mechanical stresses.

Arteries are sensitive to changes in mechanical stresses. These changes typically drive normal development but also govern disease-related vascular adaptations, e.g. in hypertension [43]. Within the arterial wall, several types of cells (i.e. vascular smooth muscle cells, fibroblasts and endothelial cells) are sensitive to an offset in mechanical stress from homeostatic values [16]. Two types of mechanical stress are relevant in this homeostatic process; wall shear stress (i.e. stress developed by friction of the blood flow at the blood-wall interface; order of magnitude of $1 \mathrm{~Pa}$ [32]) and wall stress (i.e. stress in the vessel wall developed by transmural pressure; order of magnitude of $10^{5} \mathrm{~Pa}$ [27]). As a result of changes in wall (shear) stress, cells can mediate changes in the vessel wall by 1) remodelling and/or turnover of cell products aiming to restore mechanical homeostasis, and 2) altering the vasoactive state of a vascular smooth muscle cell [16]. At the macro level, such adaptation processes affect arterial radius and wall thickness [11, 16, 43]. We hypothesise that radius and wall thickness can be estimated by incorporating adaptation rules in the PWP-model, which respond to wall stress and wall shear stress as generated by the model itself.

In the present analysis, we assess whether incorporating a model of the above described adaptation processes into a validated PWP model [23] is beneficial for model personalisation. To this end, a generic arterial tree was considered 
representing the central arteries and the arteries of the left arm. The generic tree was parametrised using generic data on arterial segment length [33] and arterial radius, wall thickness, and wall stiffness [13]. Using measured data gathered from ten young, healthy subjects, we first performed simulations using a reference PWP model fed by the entire clinical data set. Subsequently, simulations were performed using a PWP model with generic data, fed by a subset of the clinical data set. This subset contains subject-specific flow-, distensibility-, and pressure measurements, as well as a subject-specific radius and wall thickness for the left carotid arterial segment in the model. For all remaining arterial segments, radius and wall thickness were estimated by the adaptation model. Model-estimated tube geometries and pressure and flow waveforms were compared to the reference data. Additionally, a local sensitivity analysis was conducted to quantify to which extent error in the model-estimated geometries, as well as in pressure and flow waveform characteristics changed when varying the adaptation model parameters.

\subsection{Methods}

\subsubsection{Imaging and haemodynamic data}

Ten young, apparently healthy volunteers ( 5 male, $25 \pm 3$ years) were recruited to undergo ultrasound (US) and magnetic resonance imaging (MRI) examination. Exclusion criteria were smoking history or current smoking, diabetes, and the standard MRI contraindications, such as metallic devices and implants, epilepsy, pregnancy, and severe claustrophobia. The study was approved by the medical ethics committee of the Maastricht University Medical Centre and written informed consent was obtained from all volunteers before enrolment. A detailed description of data acquisition and processing steps is given in Appendix 5.A1, whilst a brief summary is given here.

Repeated oscillometric measurements of brachial blood pressure were obtained. Subsequently, applanation tonometric waveforms were obtained at the left common carotid, left brachial and left radial artery. Local carotid and radial blood dynamic pressures were obtained by calibrating tonometric waveforms, using the method of Kelly and Fitchett [21].

Two-dimensional longitudinal US recordings of the left brachial, left radial, left ulnar and left common carotid artery were acquired using a US machine equipped with a 4-13 MHz linear array probe. Diastolic diameter and distension (i.e. difference between systolic and diastolic diameter) were automatically determined using built-in wall-tracking software. Doppler-based volume flow measurements were obtained at the brachial, radial and ulnar artery. Wall thickness was measured for the carotid artery using dedicated software that automatically delineates media-adventitia echoes of near and far walls (Fig. 5.1).

Distensibility $\left(D_{0}\right)$ of the brachial, radial and carotid artery was estimated from ultrasound-based measurements of diastolic- and systolic diameters in conjunc- 
tion with the local blood pressure estimates using the relation given by

$$
D_{0}=\frac{C_{0}}{A_{0}} \approx \frac{1}{\pi r_{0}^{2}} \frac{\Delta A}{\Delta p} \approx \frac{r_{\mathrm{sys}}^{2}-r_{\mathrm{dia}}^{2}}{r_{0}^{2} \Delta p} .
$$

Here, $C_{0}$ represents area compliance and $A_{0}$ reference lumen area, expressed as $\pi r_{0}^{2}$ with $r_{0}$ is the arterial radius at mean arterial pressure. The radii $r_{\text {sys }}$ and $r_{\text {dia }}$ are the systolic and diastolic radius and $\Delta p$, is the local pulse pressure, respectively. All tonometric and ultrasound measurements were performed by a vascular research technician (J.O.R.).

Within one hour after the US exam, non-contrast enhanced MRI was performed using a 3T clinical MR system (Ingenia CX, Philips Healthcare, Best, the Netherlands). First, a 3D respiratory-navigated ECG-triggered T2-prepared turbo field echo sequence was performed, covering both the aortic arch with its branches as well as the ascending and descending aorta. After reconstructing this 3D data into coronal, sagittal, and transversal views, 2D imaging planes were selected perpendicular to the ascending aorta, brachiocephalic artery, left common carotid artery, left subclavian artery, and the descending aorta (Fig. 5.1). On each of these locations, both an anatomical scan (2D black-blood turbo spin-echo) and a quantitative flow scan (2D phase-contrast) were performed during breath-hold. The anatomical scans were used to determine the lumen diameter of the vessels, which was measured from inner edge to inner edge, in both the anterior-posterior and right-left direction, and averaged. Furthermore, for the ascending and descending aorta only, wall thickness was determined at the thinnest section of the vessel. The quantitative flow scans were acquired with 25 cardiac phases in three velocity encoding directions (right-left, anterior-posterior, and feet-head) using a velocity encoding (VENC) of $200 \mathrm{~cm} \mathrm{~s}^{-1}$. Specific details of the used MR sequences are given in Appendix 5.A1. All MR measurements were obtained by an experienced cardiac MR operator (R.J.H.), while analysis of the vessel diameters and wall thicknesses was performed by an experienced reader of the department of Cardiology (B.P.A.).

Measured subject characteristics are given in Table 5.1.

\subsubsection{Model description}

\section{Pulse wave propagation model}

The 1D PWP model computes pressure and flow waveforms within a network of blood vessels. We modelled blood vessels as linear thick-walled elastic tubes. Blood was assumed to be incompressible and Newtonian. Furthermore, gravity forces and leakage along the length of the tube were neglected. Applying conservation of mass and the momentum equations, and integrating over the vessel's cross-section yield the governing equations [15, 23]:

$$
C \frac{\partial p}{\partial t}+\frac{\partial q}{\partial z}=0
$$






Figure 5.1: Overview of the vascular imaging techniques used. A: Representation of the aortic arch, illustrating scan planes for each measured artery (i.e. ascending aorta, descending aorta, brachiocephalic, left carotid, and left subclavian artery) B: Quantitative flow images for the feet-head encoding direction (left) and anatomical images (right), as acquired for each scan plane. Arrows indicate the blood vessels of interest. C: Overview of types of vascular ultrasonography measurements. *: Estimates of carotid artery flow were acquired using MRI, whereas we chose to use ultrasonography to estimate carotid diameter.

$$
\frac{\rho}{A}\left(\frac{\partial q}{\partial t}+\frac{\partial \gamma}{\partial z}\right)+\frac{\partial p}{\partial z}=\frac{2}{r} \tau_{\mathrm{w}}
$$

Where $p=p(z, t)$ is the pressure, $q=q(z, t)$ is the flow rate, $A=A(z, t)$ is the cross-sectional lumen area and $z$ is the axial vessel distance. The parameter $C=C(z)$ denotes the vessels area compliance, constant $\rho$ is the blood density and $r$ is the radius using $r(z, t)=\sqrt{A / \pi}$. Variable $\tau_{\mathrm{w}}$ denotes wall shear stress and $\frac{\rho}{A} \frac{\partial \gamma}{\partial z}$ denotes the convection term, with $\gamma$ the axial blood velocity $\left(v_{z}\right)$ squared integrated over the cross-sectional area, i.e. $\gamma=\int_{A} v_{z}^{2} d A$. To be able to solve the above equations, we need a good approximation for $\tau_{\mathrm{w}}$ and $\frac{\partial \gamma}{\partial z}$ in terms of $p$ and $q$ in combination with a constitutive law between $A$ and $p$. For this purpose, we used the approximate velocity profile, introduced earlier [4], that approximates 
Table 5.1: Overview of characteristics and measured variables in ten subjects.

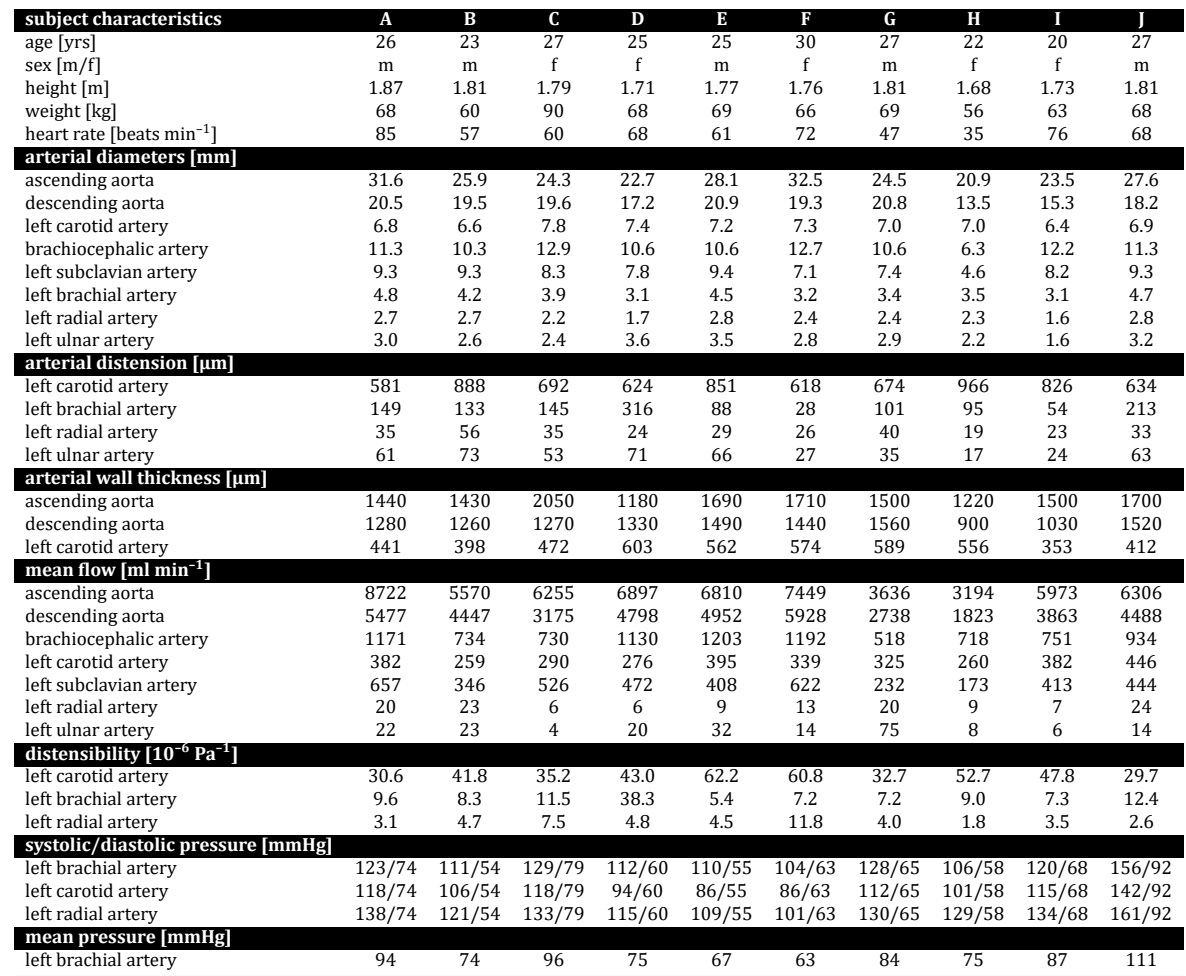

the Womersley profile in the time domain. The wall shear stress is then given by

$$
\tau_{\mathrm{w}}=-\frac{2 \eta}{\left(1-\zeta_{\mathrm{c}}\right) r} \frac{q}{A}+\frac{r}{4}\left(1-\zeta_{\mathrm{c}}\right) \frac{\partial p}{\partial z}
$$

with $\zeta_{c}=\left(\max \left[0,1-\frac{\sqrt{2}}{\alpha_{0}}\right]\right)^{2}$. Constant $\eta$ is the dynamic blood viscosity, $\zeta_{\mathrm{c}}$ is the fraction of cross-sectional area with inertia dominated flow, and $\alpha$ is the characteristic Womersley number, given by $\alpha_{0}=\sqrt{\frac{2 A_{0} \rho}{T \eta}}$. Here, $A_{0}$ denotes reference area, $p_{0}$ reference pressure, and $T$ cardiac cycle duration. For two limiting cases, given by $\alpha_{0} \ll \sqrt{2}$ and $\alpha_{0} \gg \sqrt{2}$, we obtain $\zeta_{\mathrm{c}} \approx 0$, describing a Poiseuile profile and $\zeta_{\mathrm{c}} \approx 1$, describing a flat velocity profile, respectively. Using the approximate velocity profile, $\frac{\partial \gamma}{\partial z}$ is estimated by $\frac{\partial}{\partial z}\left(\delta \frac{q^{2}}{A}\right)$. The expression for factor $\delta$ is given by Bessems et al. [4]

$$
\delta=\frac{2-2 \zeta_{\mathrm{c}}\left(1-\ln \left(\zeta_{\mathrm{c}}\right)\right)}{\left(1-\zeta_{\mathrm{c}}\right)^{2}} .
$$

In this study, compliance was assumed linear around reference pressure, $p_{0}$ (i.e. $C \approx C_{0}=\left.\frac{\partial A}{\partial p}\right|_{p=p_{0}}$ ). For a thick-walled elastic tube, an expression for $C_{0}$ was 
mathematically derived by Jager [19] and applied by Westerhof et al. [42]:

$$
C_{0}=\frac{2 \pi r_{0}^{2}\left(\frac{2 r_{0}^{2}\left(1-\mu^{2}\right)}{h_{0}^{2}}+(1+\mu)\left(\frac{2 r_{0}}{h_{0}}+1\right)\right)}{E\left(\frac{2 r_{0}}{h_{0}}+1\right)} .
$$

Constant $\mu$ is the Poisson's ratio, $E$ is the Young's modulus and $r_{0}$ and $h_{0}$ denote reference radius and wall thickness. The area change as a function of pressure can be estimated as

$$
A(z, t)=A_{0}+C_{0}\left(p-p_{0}\right)
$$

when assuming small pressure changes with respect to the reference pressure and a linearised (constant) area compliance.

\section{Governing equation for the periphery}

The contribution of the peripheral vasculature at each arterial terminus was lumped in a three-element windkessel model (Fig. 5.2). Characteristic impedance $\left(Z_{0}\right)$ represents the lossless wave impedance at the entrance of a peripheral vascular bed. Peripheral resistance $\left(R_{\mathrm{p}}\right)$ represents total resistance of a peripheral vascular bed and peripheral compliance $\left(C_{\text {art }}\right)$ represents total compliance of a peripheral vascular bed. We chose $Z_{0}$ of peripheral vascular beds that terminate the brachiocephalic artery, carotid artery and the thoracic aorta, equal to $\sqrt{L / C}$, with $L$ and $C$ respectively the inertia and compliance at the terminal node of the arterial segment. This was chosen to avoid non-physiological reflections at high frequencies (i.e. $>3$ times the heart frequency). Pressure at the end of the windkessel was kept at $0 \mathrm{mmHg}$. Furthermore, extravascular pressure of tubes was kept at $0 \mathrm{mmHg}$. Windkessel resistance $\left(R_{\mathrm{p}}\right)$ and compliance $\left(C_{\text {art }}\right)$ were based on the subject-specific flow distribution to each arterial branch, determined using a method described earlier [29].

The differential equation governing the relation between pressure and flow is given by [33]:

$$
\frac{\partial q}{\partial t}=\frac{1}{Z_{0}} \frac{\partial p}{\partial t}+\frac{p}{Z_{0} R_{\mathrm{p}} C_{\text {art }}}-\left(1+\frac{Z_{0}}{R_{\mathrm{p}}}\right) \frac{q}{Z_{0} C_{\text {art }}} .
$$

Previous works chose distal $Z_{0}$ based on a fixed fraction of $R_{\mathrm{p}}$, or used a single resistor to terminate small arterial branches $[33,41]$. Here, we explicitly assumed $Z_{0} \ll R_{\mathrm{p}}$ by fixing $Z_{0}$ at $10^{-2} \mathrm{~Pa} \mathrm{~s} \mathrm{~m}{ }^{-3}$ for peripheral elements terminating the vertebral, radial, ulnar and interosseous artery [1].

\section{Adaptation model}

The adaptation model is based on two phenomena, as observed in vascular physiology. On the one hand, we take into account wall thickness adaptation due to changes in wall stress. Earlier work showed that increased wall stress due to 


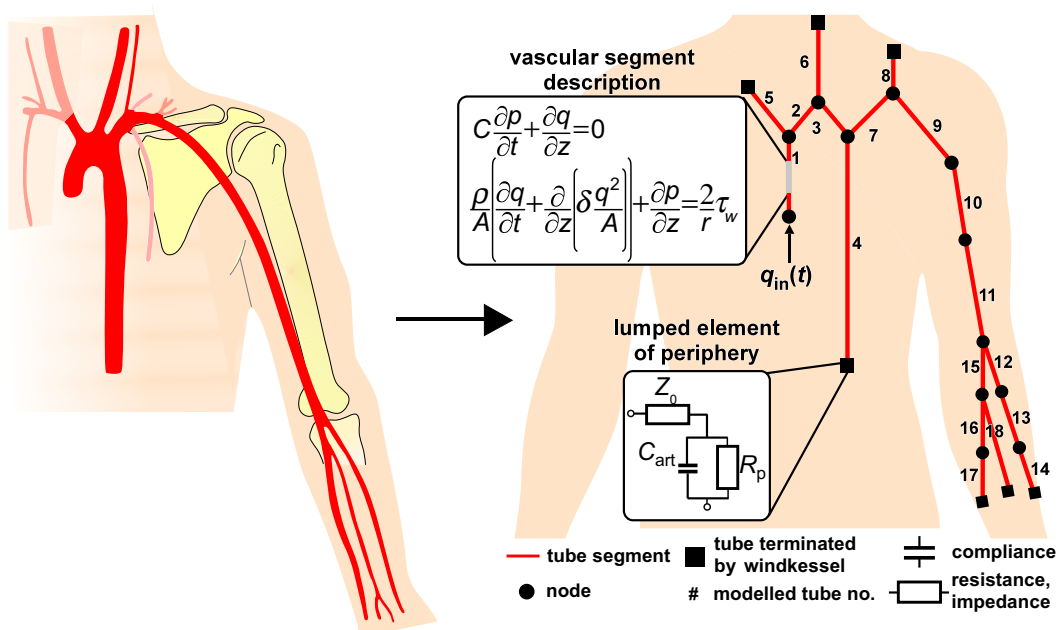

Figure 5.2: Overview of modelled domain describing the arteries of the aorta and carotid, and the arteries of the left arm. Flow rate $\left(q_{\text {in }}(t)\right)$ was prescribed to the proximal node of the arterial domain. For visualisation purposes the number of nodes displayed per modelled tube is reduced, displaying only the proximal and distal node per tube. Generic tube properties can be retrieved from Table 5.2.

induced hypertension in rats is associated with a progressive increase in wall thickness, eventually resulting in normalisation of wall stress to that during a normotensive state [43].

The increase in wall thickness has been attributed to vascular smooth muscle cell hypertrophy, hyperplasia, and increased amounts of elastin and collagen [27, 43]. On the other hand, we take into account vessel radius adaptation due to changes in wall shear stress [20]. These changes are sensed by endothelial cells which modulate vascular smooth muscle cell tone, resulting in vasoconstriction or vasodilation through nitric oxide production or washout [16, 24]. If changing vascular tone is insufficient to normalise wall shear stress, turnover of cell products will cause the artery to structurally adapt [22].

\section{Vessel wall thickness adaptation}

We modelled wall thickness adaptation based on the following hypothesis. Crosssectional wall area $\left(A_{\text {wall }}\right)$ was changed in response to a relative deviation of peak wall stress $\left(\sigma_{\mathrm{f}}\right)$ from a target peak wall stress $\left(\sigma_{\mathrm{f}, \text { target }}\right)$. The change in $A_{\text {wall }}$ is described by the following equation:

$$
A_{\mathrm{wall}, n+1}=\left(\frac{\sigma_{\mathrm{f}, n}}{\sigma_{\mathrm{f}, \mathrm{target}}}\right)^{k_{\mathrm{A}}} \cdot A_{\mathrm{wall}, n} .
$$


Table 5.2: Generic values of modelled arterial segments [13, 33].

\begin{tabular}{llllllll}
\hline tube \# & arterial segment & $l[\mathrm{~cm}]$ & $r_{\text {proximal }}[\mathrm{mm}]$ & $r_{\text {distal }}[\mathrm{mm}]$ & $h[\mathrm{~mm}]$ & $E\left[10^{5} \mathrm{~Pa}\right]$ & $\bar{q}\left[\mathrm{ml} \mathrm{min}^{-1}\right]$ \\
\hline 1 & ascending aorta & 4.00 & 14.70 & 14.70 & 1.47 & 4.0 & $5.2 \cdot 10^{3}$ \\
2 & aortic arch A prox. & 2.00 & 12.60 & 12.00 & 1.23 & 4.0 & \\
3 & aortic arch A distal & 3.90 & 10.70 & 10.40 & 1.06 & 4.0 & \\
4 & aortic arch B + thoracic aorta & 15.60 & 10.00 & 6.50 & 1.91 & 4.0 & $4.2 \cdot 10^{3}$ \\
5 & brachiocephalic & 3.40 & 10.10 & 9.00 & 0.82 & 4.0 & 470 \\
6 & left carotid & 9.10 & 6.80 & 3.50 & 0.51 & 4.0 & 360 \\
7 & left subclavian & 3.40 & 5.80 & 4.50 & 0.51 & 4.0 & \\
8 & left vertebral & 14.90 & 1.90 & 0.90 & 0.21 & 8.0 & 26 \\
9 & left subcl.+axill.+brach. prox. & 14.77 & 4.10 & 3.60 & 0.57 & 4.0 & \\
10 & left brachial middle & 14.77 & 3.60 & 3.10 & 0.50 & 4.0 & \\
11 & left brachial distal & 14.77 & 3.10 & 2.40 & 0.55 & 4.0 & \\
12 & left radial proximal & 7.83 & 1.90 & 1.80 & 0.36 & 8.0 & \\
13 & left radial middle & 7.83 & 1.80 & 1.70 & 0.34 & 8.0 & \\
14 & left radial distal & 7.83 & 1.70 & 1.60 & 0.32 & 8.0 & 40 \\
15 & left ulnar proximal & 6.70 & 1.90 & 1.70 & 0.36 & 8.0 & \\
16 & left ulnar middle & 7.90 & 1.60 & 1.50 & 0.31 & 8.0 & \\
17 & left ulnar distal & 8.55 & 1.50 & 1.40 & 0.29 & 8.0 & 30 \\
18 & left interosseous & 8.55 & 1.10 & 0.90 & 0.20 & 16.0 & 30 \\
\hline
\end{tabular}

$l$ : length of the arterial segment, $r_{\text {proximal }}:$ proximal radius, $r_{\text {distal }}:$ distal radius, $h$ : wall thickness, and $E$ : is the Young's modulus. Mean flow rate $\bar{q}$ represents either the proximal inflow or the terminal flow assigned to the peripheral elements of the model.

Where $n$ denotes the current adaptation cycle and constant $k_{A}>0$ governs the rate of adaptation. Note that vessel wall thickness $(h)$ follows from cross-sectional wall area and vessel radius using $h=\sqrt{A_{\text {wall }} / \pi+r^{2}}-r$. According to our adaptation rule, wall thickness is determined by the maximum pressure $\left(p_{\max }\right)$, being the sum of the component due to peak pressure and the component related to whole-body acceleration, e.g. occurring during moderate jumping. The value of $\sigma_{\text {f,target }}$ was chosen equal to $400 \mathrm{kPa}$ so that the resulting wall thickness in the circulatory system is physiologic [3]. The expression for $p_{\max }$ is obtained, assuming that inertia forces dominate over viscous forces:

$$
p_{\max }=\max (p)+\max \left(Z_{0} \cdot A\right) \cdot v_{\text {impact }},
$$

with $p$ the blood pressure, $Z_{0}$ the vessel's characteristic impedance and $A$ the vessel's lumen area. As a first-order approximation, we assume that a body jump from $0.5 \mathrm{~m}$ height will induce an additional impact on the vessel wall that still can be resisted. We based this height on earlier works studying the human vertical jumping agility [2]. At impact, assuming no initial velocity and drag, body velocity $v_{\text {impact }}$ is $\approx \sqrt{2 g h} \approx 3.0 \mathrm{~m} \mathrm{~s}^{-1}$. Now we have found a first order approximation of $p_{\text {max }}$, we are able to derive an expression for $\sigma_{\mathrm{f}}$. Assuming blood vessels to be fibre-reinforced shells for which homogeneity of mechanical load within the wall holds, wall stress $\left(\sigma_{\mathrm{f}}\right)$ can be related to internal pressure $(p)$ and the ratio of cavity to wall volume $\left(\mathrm{V} / \mathrm{V}_{\text {wall }}\right)$ using the expression given by Arts et al. [3]

$$
\sigma_{\mathrm{f}}=\beta_{1} p_{\max }\left(\frac{V}{V_{\text {wall }}}+\beta_{2}\right)
$$


where $\beta_{1}$ and $\beta_{2}$ are dimensionless constants. We adopted $\beta_{1}=3$ and $\beta_{2}=0.5$ based on Arts et al. [3]. Substituting Eq. 5.10 in Eq. 5.11 and dividing by the length of the vessel segment, yields the following expression for $\sigma_{\mathrm{f}}$ :

$$
\sigma_{\mathrm{f}}=3\left(\max (p)+\max \left(Z_{0} \cdot A\right) \cdot v_{\text {impact }}\right)\left(\frac{\max (A)}{A_{\text {wall }}}+\frac{1}{2}\right) \text {. }
$$

\section{Vessel radius adaptation}

We assumed changes in mean wall shear stress to be the driving factor for vessel radius adaptation. An expression for mean wall shear stress is given by

$$
\bar{\tau}_{\mathrm{w}}=\frac{4 \eta \bar{q}}{\pi r^{3}},
$$

with $\bar{q}$ the mean flow rate. Vessel radius $(r)$ was changed in response to a relative deviation of mean wall shear stress $\left(\bar{\tau}_{\mathrm{w}}\right)$ from a target wall shear stress $\left(\bar{\tau}_{\mathrm{w} \text {,target }}\right)$ :

$$
r_{n+1}=\left(\frac{\bar{\tau}_{\mathrm{w}, n}}{\bar{\tau}_{\mathrm{w}, \text { target }}} \cdot\left(1+r_{\text {crit }} / r_{n}\right)\right)^{k_{r}} \cdot r_{n} .
$$

Here, $k_{r}>0$ is the adaptation rate constant for radius adaptation. We considered separate target wall shear stress values for central (denoted $\bar{\tau}_{\mathrm{w} \text {,target,prox }}$ ) and distal (denoted $\bar{\tau}_{\mathrm{w} \text {,target,dist }}$ ) arteries. For central arteries, represented in the model by tubes \#1 to \#8, we chose $\bar{\tau}_{\text {w,target,prox }} 0.3 \mathrm{~Pa}[6,38]$. For distal arteries, represented in the model by tubes \#9, \#10 and \#12 to \#18, $\bar{\tau}_{\mathrm{w} \text {,target,dist }}$ was chosen $0.4 \mathrm{~Pa}[9,32]$. The ratio $r_{\text {crit }} / r_{n}$ is added to capture the fact that the vessel radius dependency on wall shear stress might reduce for vessels having a radius smaller than the critical lumen radius. Critical lumen radius $\left(r_{\text {crit }}\right)$ was estimated from earlier reports on vascular smooth muscle cell length and their organisation within the arterial wall, and set to $0.45 \mathrm{~mm}$ [28].

This radius dependency of vessel dilatation in response to wall shear stress could hypothetically be explained via principles of tensegrity. According to this principle, tension in fibres embedded in tissues, bear forces that may strengthen the structure of the wall by compression of soft material like fluids and cells between these fibres [18].

For example, contraction of a smooth muscle cell in the outer media pulls on the network of collagen and elastin fibres that extends into the adventitia, thus pulling collagen and elastin fibres from the adventitia into the media.

Consequently, a small region of the adventitia near the attachment point of the smooth muscle cell will squeeze. After relaxation of the smooth muscle cell, a fraction of the deformation remains, assumedly due to cross-linking of collagen fibres under tension (tension-fixation) [18].

In our model of radius adaptation, we assume that pulling of collagen and elastin fibres from adventitia into medial direction is proportional with the radial component $\left(F_{r}\right)$ of smooth muscle cell force $\left(F_{\mathrm{SMC}}\right)$. In large blood vessels, force 


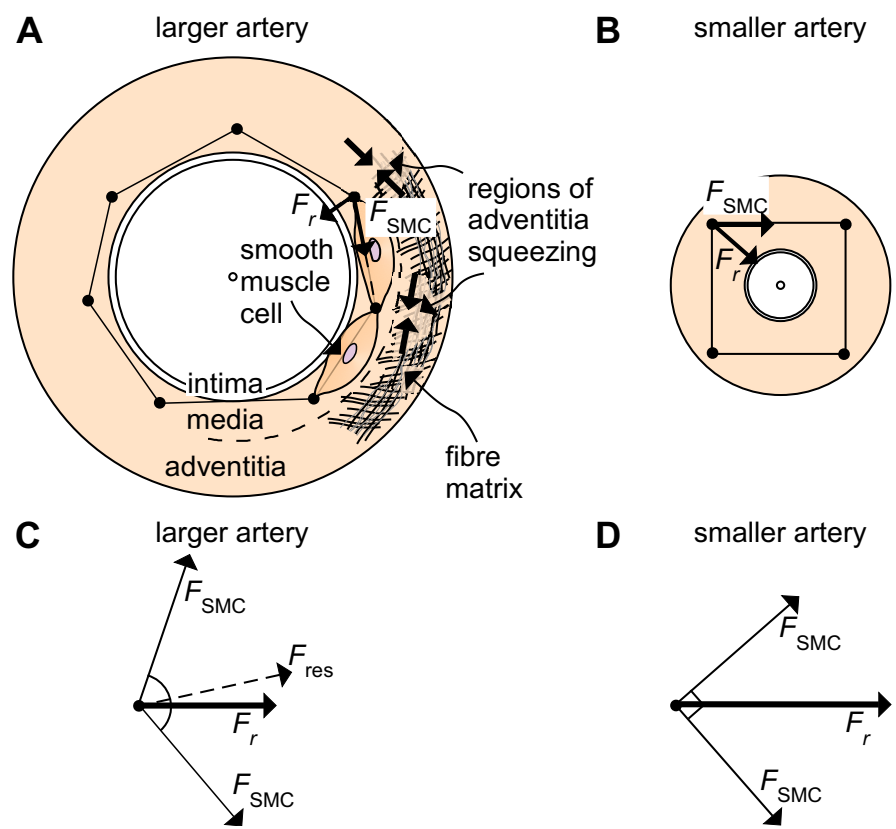

Figure 5.3: Schema of the hypothesised radius dependency of vessel dilatation, distinguishing a larger artery (A) and a smaller artery (B). Nodes represent attachment points of SMCs. We hypothesised that SMC contraction causes pulling of elastic material from the adventitia into the medial layer, squeezing the adventitia. SMC force, indicated by the black straight lines, follow the vessel's curvature. Hence, the radial force component $\left(F_{r}\right)$ of SMC force $\left(F_{\mathrm{SMC}}\right)$ is inversely related to vessel radius, i.e. smaller for larger arteries (C) than smaller arteries (D). In $\mathrm{C}$, the resultant force vector $\left(F_{\text {res }}\right)$ differs from the radial force vector $\left(F_{r}\right)$ and in D, vectors $F_{\text {res }}$ and $F_{r}$ are the same. Note that $F_{\text {SMC }}$ was assumed equal for larger arteries and smaller arteries, and that wall thickness is exaggerated in this figure.

pathways sprouting from the ends of smooth muscle cells are more parallel to the wall. Consequently, $F_{r}$ is inversely proportional with vessel radius (Fig. 5.3 A, C). For sufficiently small vessels, the force field around a smooth muscle cell follows the curvature of vessel wall geometry, so that the radial component of force is less dependent on vessel radius (see Fig. 5.3 B, D). Extension rate $\left(\frac{1}{r} \frac{\partial r}{\partial t}\right)$ of the blood vessel is thus assumed to depend on vessel radius according to

$$
\frac{1}{r} \frac{\partial r}{\partial t}=\left(F_{\mathrm{ext}}-\frac{F_{\mathrm{SMC}}}{1+r / r_{\text {crit }}}\right)
$$

where $F_{\text {ext }}$ is a background extension force in absence of smooth muscle cell contractions. 


\section{Numerical implementation}

Coupling and solving of 1D mass conservation and momentum balance equations (i.e. Eqs. 5.2 and 5.3) and the 0D equation for pressure and flow in the periphery (Eq. 5.8) was performed using a previously published method [23]. We used a simplified trapezoidal scheme for spatial discretisation. The second-order backward difference scheme was used for time discretisation. We chose an element size (i.e. $\Delta z$, indicating the distance between nodes) of $0.01 \mathrm{~m}$, since this resulted in adequate numerical convergence [23]. A schematic overview of the PWP-adaptation framework is shown in Fig. 5.4.

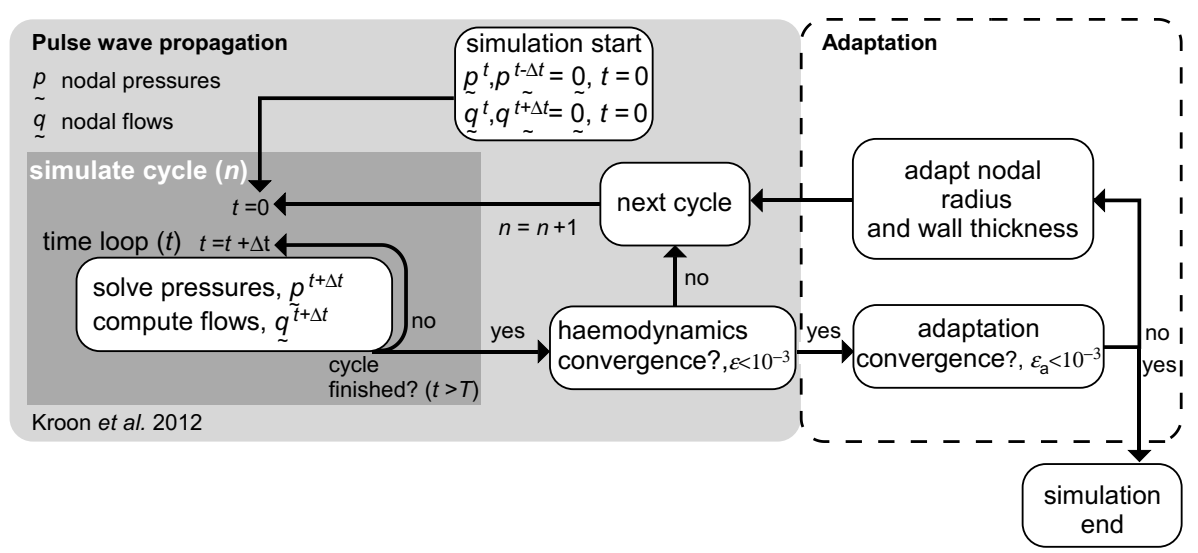

Figure 5.4: Schematic overview of the PWP-adaptation framework. We used a time loop for simulating a single cardiac cycle with cycle duration $T$ and time step $\Delta t$. The time loop was continued until haemodynamics convergence (defined as $\epsilon<$ $10^{-3}$ ) was achieved, indicating a steady-state simulation. Upon haemodynamics convergence, adaptation convergence was evaluated (i.e. $\epsilon_{\mathrm{a}}<10^{-3}$ ). Nodal radius and wall thickness were adapted until adaptation convergence was achieved, indicating normalisation of wall stress and wall shear stress to target values.

Nodal pressures were solved for globally and nodal flows were subsequently computed from the pressures on element level (Fig. 5.4). The method used for solving the system of $0 \mathrm{D}$ - and $1 \mathrm{D}$ equations has been tested earlier in a benchmark study, showing reliable results [5]. Convergence of the calculated haemodynamics was evaluated after each simulated cardiac cycle. Hereto, the calculated nodal pressures, $p(t)$, of the current cycle $(n)$ were compared to those of the previous cycle $(n-1)$. An expression for the haemodynamics convergence norm $(\varepsilon)$ is given as a relative root-mean-square:

$$
\epsilon=\max _{i}\left(\sqrt{\frac{\sum_{t=0}^{T}\left(p_{i, n}^{t}-p_{i, n-1}^{t}\right)^{2}}{\sum_{t=0}^{T}\left(p_{i, n-1}^{t}\right)^{2}}}\right),
$$


where $i$ is the nodal point number. Following haemodynamics convergence, arterial radius and wall thickness of nodes were adapted based on the adaptation rules. For the next cycle $(n=n+1)$, it was evaluated whether $\varepsilon$ was still below the convergence criterion and whether the model-estimated wall stress and mean wall shear stress had converged to their respective target values (Fig. 5.4). Adaptation convergence was evaluated by calculating the relative error of wall stress and wall shear stress given by

$$
\epsilon_{\mathrm{a}}(\bullet)=\max (|\bullet-1|),
$$

with $(\bullet)=\left\{\frac{\sigma_{\mathrm{f}, N, i}}{\sigma_{\mathrm{f}, \max }}, \frac{\bar{\tau}_{\mathrm{w}, N, i}}{\bar{\tau}_{\mathrm{w}, \text { target }}}\right\}$. Here, $\sigma_{\mathrm{f}, N, i}$ and $\bar{\tau}_{\mathrm{w}, N, i}$ denote the wall stress and mean wall shear stress values for tube $N$. We kept the haemodynamics- and adaptation convergence criteria at $10^{-3}$ to ensure complete adaptation. In Fig. 5.5, a representative example of adaptation of tube radius and wall thickness is shown.
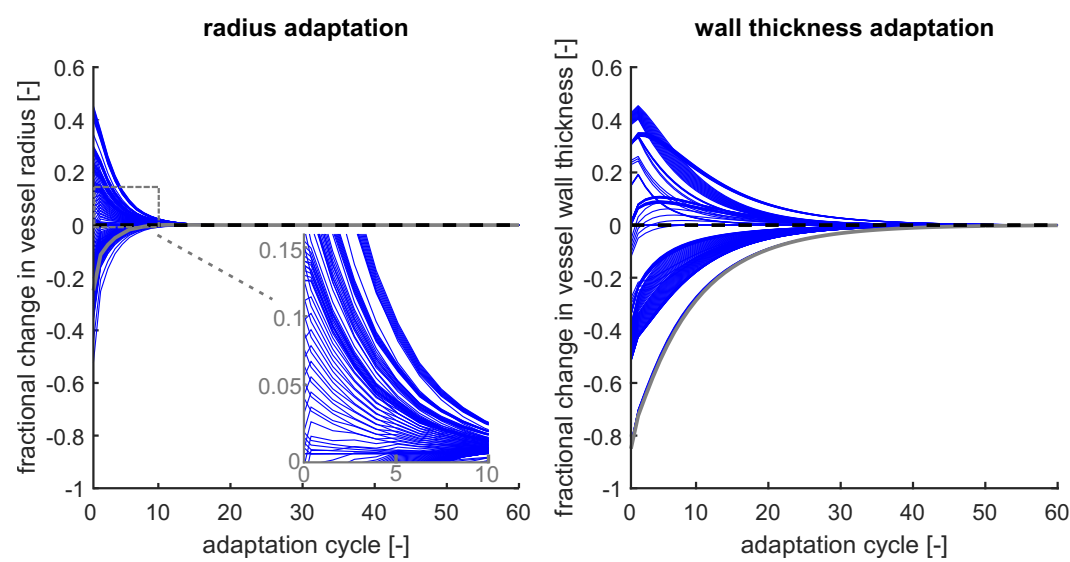

Figure 5.5: Adaptation of vessel radius (left) and wall thickness (right) for all modelled vascular segments. The fractional change of vessel radius and wall thickness is plotted as a function of the number of adaptation cycles following departure from generic values. The grey curve for vessel radius and wall thickness highlights the adaptation behaviour of a randomly chosen vascular segment.

Since we were only interested in the adapted geometries and not in the adaptation time-course, feedback factors $k_{A}$ and $k_{r}$ were kept at 0.10 to ensure on the one hand numerical stability and to reduce on the other hand computational cost. Correspondingly, adaptation convergence was reached after approximately 50 cycles.

We performed all model simulations and processing steps using MATLAB 2015a (The Mathworks, Natick, MA, USA). For all simulations, the time step $(\Delta t)$ was kept at $1 \mathrm{~ms}$. Following previous studies, density of blood $(\rho)$ was chosen $1050 \mathrm{~kg}$ $\mathrm{m}^{-3}$, dynamic blood viscosity $(\eta)$ was chosen $3 \cdot 10^{-3}$ Pa s and Poisson's ratio $(\mu)$ was chosen 0.5 , indicating incompressibility of the vessel wall $[23,42]$. 


\subsubsection{Simulations and analysis}

To assess the potential of our approach in completing sparse data sets, we performed three sets of simulations: 1) the PWP model without adaptation, but fed with the complete clinical data set; 2) the PWP model including adaptation, using a subset of the clinical data as input; and 3) simulations to evaluate the effect of adaptation model parameters on output metrics. Details of these three analyses are given below.

\section{RefModel}

To obtain the reference PWP models (referred to as the REFModEL, Fig. 5.6), we prescribed for each subject:

1. The radius and wall thickness of all 18 modelled tubes. For measured segments, we imputed measured values. For arterial segments that were not measured, we obtained estimates by applying a subject-specific scaling for radius (Table 5.2) and a more generalised scaling rule for wall thickness. For tube radius, we calculated a subject-specific scaling factor determined from the ratio between the measured diameter of the left subclavian artery and its generic value from Reymond et al. [33] (Table 5.2). To estimate wall thickness, we used vessel-specific ratios between wall thickness and radius (i.e. the ratio $h / r$ ), assumed 0.10 for the aortic segments, 0.15 for the brachiocephalic, vertebral artery, and brachial artery, and 0.20 for the radial and interosseous artery [13].

2. Ascending aortic flow rate $\left(q_{\text {in }}(t)\right)$, obtained using PC-MRI, to the proximal node of tube \#1.

3. The flow distribution to the periphery, as derived from PC-MRI and Doppler measurements. Since flow measurements for the interosseous artery were absent, we assigned the same flow fraction to the interosseous artery as for the ulnar artery.

4. Reference pressure $\left(p_{0}\right)$ using the mean pressure measured at the brachial artery.

5. The Young's modulus of the subclavian, axillary, brachial, ulnar, radial and interosseous artery. Using Eq. 5.6, the measured $D_{0}$ was translated to a Young's modulus, to obtain material stiffness values for the brachial, radial, and carotid arterial walls. For the subclavian and axillary arteries, we imputed the Young's modulus of the brachial artery, whereas for the ulnar and interosseous arteries in the model, we imputed the Young's modulus of the radial artery.

All other model parameters were kept at generic values as given in Table 5.2. 


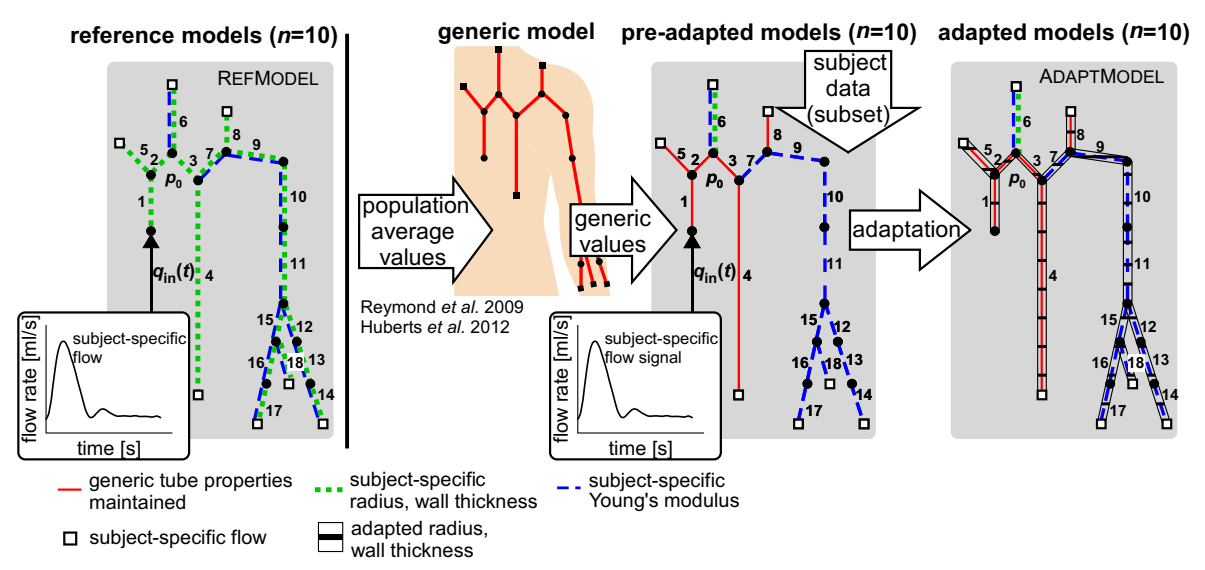

Figure 5.6: Schematic overview of the simulation protocol used for obtaining the REFMODEL and ADAPTMODEL of each subject. The REFMODEL was obtained by incorporating the entire measured data set in the generic model in one step. The ADAPTMODEL was obtained in two steps. Here, $p_{0}$ denotes the subject-specific mean pressure and $q_{\text {in }}$ the subject-specific inflow profile.

\section{AdaptModel}

To obtain the adapted PWP models, referred to as the ADAPTModEL (Fig. 5.6), from the generic model (Table 5.2) we used the following approach.

First, we prescribed the subset of the data, as described in 2 to 5 in the previous section, and in addition the radius and wall thickness of the left carotid artery, to obtain pre-adapted models (Fig. 5.6). We then initiated the PWP-adaptation procedure on the pre-adapted models, to estimate vessel radius and wall thickness for all arteries in the model except the left carotid artery.

\section{Supporting simulations}

First, to evaluate whether radius and wall thickness adaptation resulted in unique vessel geometries, ten sets of initial vessel geometries were randomly generated. Random vessel geometries were generated by multiplying the generic vessel geometries (Table 5.2) with random values, obtained using MATLAB's normal random number generator (i.e. normrnd (mu, sd), with $\mathrm{mu}=1.0$ and sd $=0.15$, respectively). To assess uniqueness, two coefficients of variation for tube radius and wall thickness were calculated (i.e. $V_{r}=\sigma_{r} / \mu_{r} \cdot 100 \%$ and $V_{h}=\sigma_{h} / \mu_{h} \cdot 100 \%$, respectively). Here, $\sigma_{r}$ and $\sigma_{h}$ denote respectively the standard deviation of the adapted tube radii or wall thicknesses and $\mu_{r}$ and $\mu_{h}$ denote mean take-off values.

Second, we compared pressure and flow waveforms generated by the ADAPTMOdel to waveforms generated by the REFModel. Since the reference data used 
for the REFMoDEL itself is hampered by measurement error, an envelope of realisations of reference PWP models were obtained, based on assumed measurement errors of $\pm 10 \%$ for radius and $\pm 40 \%$ for wall thickness [26]. Using Latin hypercube sampling, a total of forty samples of reference radius and wall thickness were drawn and incorporated into the REFMoDEL [36].

Third, a local sensitivity analysis by varying adaptation model parameters by $\pm 25 \%$ around their initial target values was performed to assess how pressure waveform characteristics changed. We selected the following characteristics to assess sensitivity to parameter variance (i.e. $\sigma_{\text {f,target }}, \bar{\tau}_{\mathrm{w} \text {,target,prox }}, \bar{\tau}_{\mathrm{w} \text {,target,dist }}, r_{\text {crit }}$ and $\left.v_{\text {impact }}\right)$ :

- Aortic systolic- and diastolic pressure ( $p_{\mathrm{s}, \text { aorta }}$ and $p_{\mathrm{d} \text {,aorta }}$, respectively)

- Radial systolic- and diastolic pressure ( $p_{\mathrm{s}, \text { radial }}$ and $p_{\mathrm{d} \text {,radial }}$, respectively)

- Pulse pressure amplification $\left(\Delta p_{\mathrm{amp}}\right)$

- Carotid-to-radial pulse wave velocity $\left(c_{\text {pulse }}\right)$

Output $\Delta p_{\text {amp }}$ was defined as the ratio between radial pulse pressure and aortic pulse pressure, according to established methods [25]. Output $c_{\text {pulse }}$ was obtained by dividing the path length between the terminal nodes of the tubes that modelled the carotid artery and radial artery by the pulse transit time between these nodes. The sensitivity $(S)$ of output metric $(y)$ to adaptation parameter $(x)$ was calculated as $S=(\Delta y / y) /(\Delta x / x) \cdot 100 \%$.

\section{Metrics for agreement between AdaptModel and RefModel}

Agreement between model-estimated and reference radius and wall thickness, as well as between ADAPTMODEL and REFMoDEL pressure and flow waveforms was quantified using the root-mean-square errors given by

$$
\begin{gathered}
\epsilon_{r}=\sqrt{\frac{\sum_{i=1}^{N}\left(r_{\mathrm{ref}}-r_{\mathrm{mod}}\right)^{2}}{N}}, \quad \epsilon_{p}=\sqrt{\frac{\sum_{t=0}^{T}\left(p_{\text {ref,median }}(t)-p_{\mathrm{mod}}(t)\right)^{2}}{(T / \Delta t)}}, \\
\epsilon_{h}=\sqrt{\frac{\sum_{i=1}^{N}\left(h_{\mathrm{ref}}-h_{\mathrm{mod}}\right)^{2}}{N}}, \quad \epsilon_{q}=\sqrt{\frac{\sum_{t=0}^{T}\left(q_{\mathrm{ref}, \text { median }}(t)-q_{\mathrm{mod}}(t)\right)^{2}}{(T / \Delta t)}} .
\end{gathered}
$$

Here, $r_{\text {mod }}$ and $h_{\text {mod }}$ represent model-estimated radius and wall thickness, and $r_{\text {ref }}$ and $h_{\text {ref }}$ represent reference radius and wall thickness, respectively. Furthermore, $p_{\text {mod }}$ and $q_{\text {mod }}$ represent model-estimated pressure and flow waveforms. The waveforms $p_{\text {ref,median }}$ and $q_{\text {ref,median }}$ represent reference pressure and flow waveforms, calculated as the median of the forty REFMoDEL realisations. Term $(T / \Delta t)$ denotes the number of data points per cycle and $N$ the number of tubes. Only tube geometries based on actual measurements were included in the calculation of $\epsilon_{r}$ (i.e. tubes \#1,\#4,\#5, \#7, \#10,\#13, and \#16) and $\epsilon_{h}$ (i.e. tubes \#1 and \#5). 


\subsection{Results}

\subsubsection{Convergence to unique vessel geometry}

Departing from ten sets of take-off geometries around values given in Table 5.2, our adaptation framework resulted in negligible variations in adapted radius and wall thickness. The coefficient of variation $\left(V_{r}\right)$ for tube radius were $\leq 4 \cdot 10^{-4} \%$, whereas for wall thickness we found $V_{h} \leq 2 \cdot 10^{-1} \%$.

\subsubsection{Agreement between model-estimated and reference geometry}

Fig. 5.7 illustrates for two subjects the comparison between reference radius and wall thickness (black squares and whiskers) and model estimations of radius and wall thickness (red asterisks). For the subjects presented in Fig. 5.7, errors in
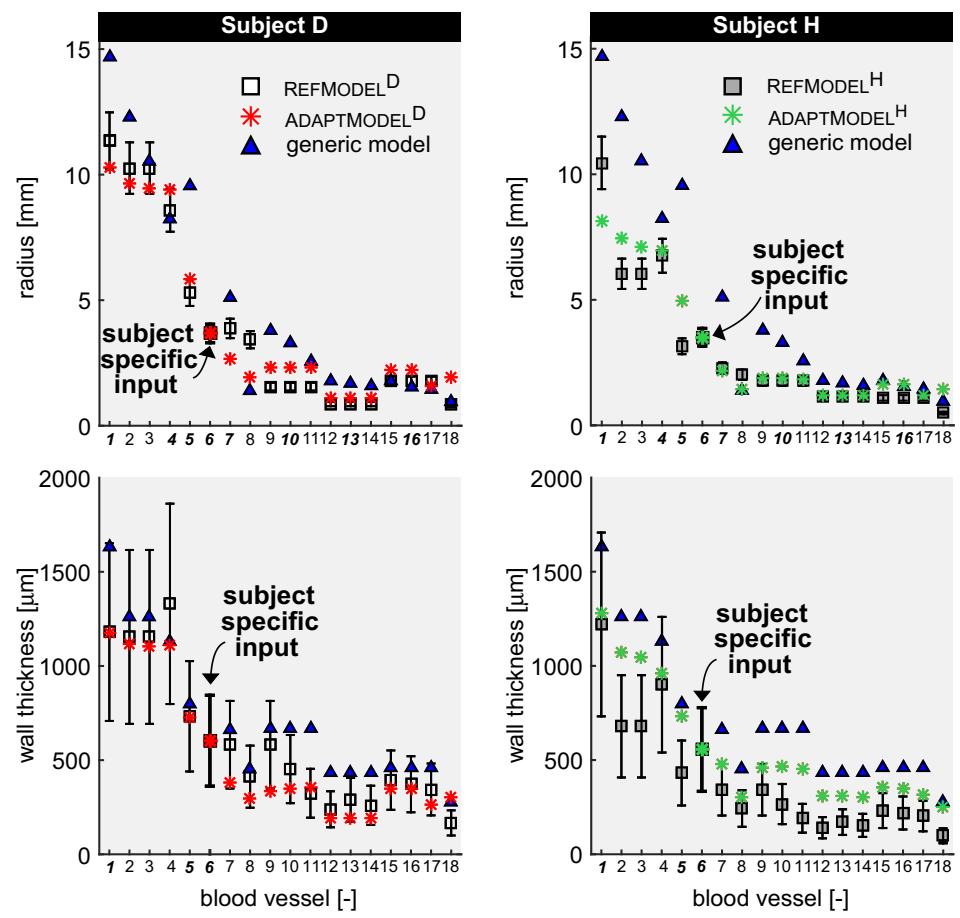

Figure 5.7: Comparison of the reference radius and wall thickness $\left(\operatorname{REFModEL}^{\mathrm{D}}\right.$ and REFMODEL $^{\mathrm{H}}$, squares with whiskers, respectively), generic values (triangles) and model-estimated tube geometries (ADAPTMODEL ${ }^{\mathrm{D}}$ and ADAPTMODEL ${ }^{\mathrm{H}}$, asterisks) for subject $\mathrm{D}$ and $\mathrm{H}$, respectively. Whiskers indicate assumed measurement uncertainty. Blood vessel numbers correspond to those depicted in Fig 5.2. Bold italic values on the horizontal axes refer to vessels for which actual vessel radius and wall thickness measurements were obtained. 
estimation of radius and wall thickness were small as evidenced by $\epsilon_{r}$ of 0.83 and $1.13 \mathrm{~mm}$, and $\epsilon_{h}$ of 136 and $150 \mu \mathrm{m}$, respectively. The radius and wall thickness of proximal arteries, representing the segments of the aorta were also reasonably captured by the adaptation framework (Fig. 5.7). In Fig. 5.8, agreement between estimated radius and wall thickness on the one hand and reference data on the other hand is shown for all subjects $(n=10)$. Agreement was quantified only for
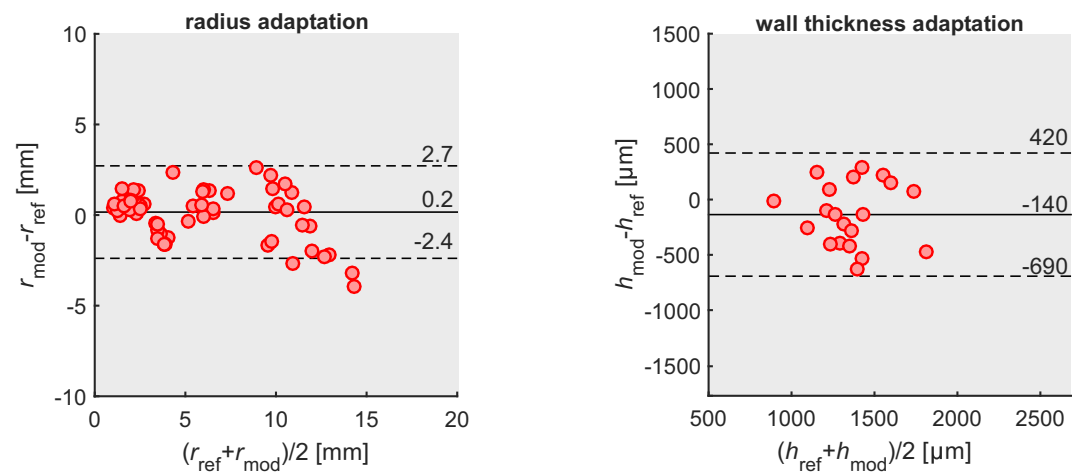

Figure 5.8: Bland-Altman plots of agreement between model-estimated and measured radius $(r)$ and wall thickness $(h)$. Values denote bias and limits of agreement (i.e. bias $\pm 2 \mathrm{SD}$ of difference) between model estimations and measured data, respectively.

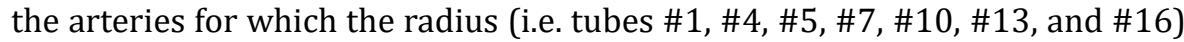
and wall thickness (i.e. tubes \#1 and \#5) were measured. Limits of agreement (i.e. bias \pm 2 SD of the difference) were $0.2 \pm 2.6 \mathrm{~mm}$ for radius and $-140 \pm 557 \mu \mathrm{m}$ for wall thickness.

Additionally, the agreement between generic tube geometries (Table 5.2) and subject data was inferior to that of the model-estimated ones, as described by limits of agreement of $1.6 \pm 3.4 \mathrm{~mm}$ for radius and $0.5 \pm 624 \mu \mathrm{m}$ for wall thickness, respectively.

\subsubsection{Pressure and flow waveforms}

Fig. 5.9 exemplifies by subject A the results on pressure $(p)$ and flow $(q)$ waveforms of the aorta, carotid artery and radial artery. Pressure and flow waveform characteristics, (i.e. mean, diastolic- and systolic values) generated by the ADAPTMODEL ${ }^{\mathrm{A}}$ were in agreement with the ranges found using the REFMoDEL ${ }^{\mathrm{A}}$. Furthermore, for central arteries, $p$ and $q$ waveforms appear similar compared to median reference waveforms $\left(\epsilon_{p}\right.$ equal to $1.5 \mathrm{mmHg}$ and $1.4 \mathrm{mmHg}$ and $\epsilon_{q}$ equal to $7.6 \mathrm{ml} \mathrm{s}^{-1}$ and $0.3 \mathrm{ml} \mathrm{s}^{-1}$, Fig. 5.9). For the distal segment of the radial artery, $p$ and $q$ waveforms increasingly differed from median reference waveforms $\left(\epsilon_{p}=9.2 \mathrm{mmHg}\right.$ and $\epsilon_{q}=0.1$ $\mathrm{ml} \mathrm{s}^{-1}$, Fig. 5.9). For the pre-adapted model, fed with subject-specific data but with generic vascular geometries, pressure and flow waveform (characteristics) 

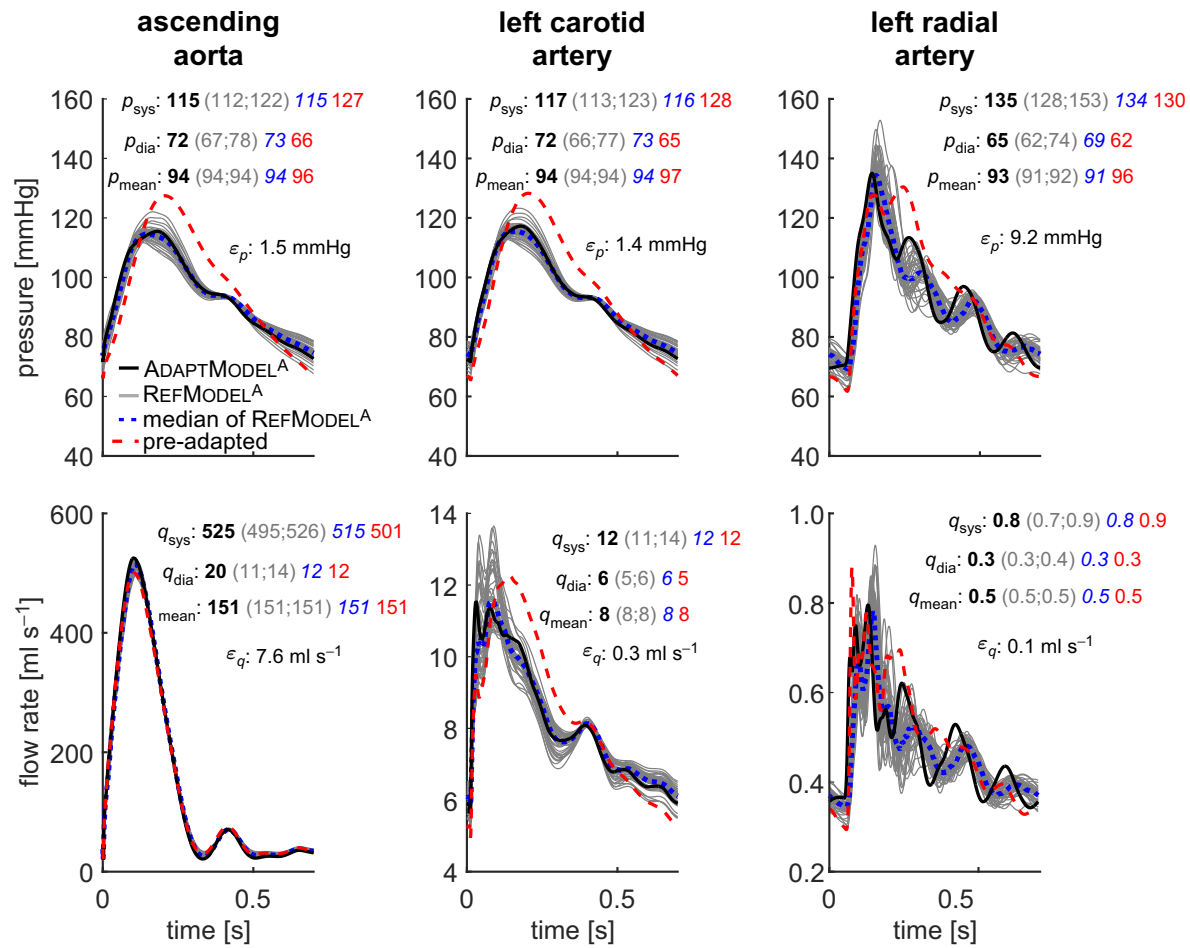

Figure 5.9: Pressure $(p)$ and flow $(q)$ waveforms for subject $A$ at three locations, i.e. the aortic arch (left panel), left carotid artery (centre panel) and left radial artery (right panel). The $p$ and $q$ waveforms generated by the ADAPTMODEL are indicated by the black curves. The $p$ and $q$ waveforms generated by the REFMODEL are indicated by the grey curves, respectively. The median $p$ and $q$ waveforms of the reference waveforms are indicated by the blue dotted curves. The $p$ and $q$ waveforms generated by the pre-adapted model (i.e. with the vessels not adapted) are indicated by the red dashed curves. Systolic-, diastolic- and mean pressures are displayed for waveforms generated by the ADAPTMODEL (bold values, black), the REFMoDEL (ranges, grey) and for the median of all reference waveforms (italic values, blue) and the pre-adapted model (red). Root-meansquare errors between adapted- and median pressure waveforms, as well as flow waveforms are described as $\epsilon_{p}$ and $\epsilon_{q}$, respectively.

significantly differed from the REFMODEL ${ }^{\mathrm{A}}$.

For all subjects, pressure and flow waveforms generated by the ADAPTMODEL, were in better agreement with reference pressure and flow waveforms, as compared to those generated by the pre-adapted model. This is illustrated by the significant reduction of on the one hand $\epsilon_{p}$ at the aorta by $49 \%$ (i.e. from 7.4 to $3.8 \mathrm{mmHg}$ ), and on the other hand the reduction in $\epsilon_{q}$ by $36 \%$ (i.e. from 7.8 to 5.0 $\mathrm{ml} \mathrm{s}^{-1}$ ), when adapting the vessels (Fig. 5.10). 

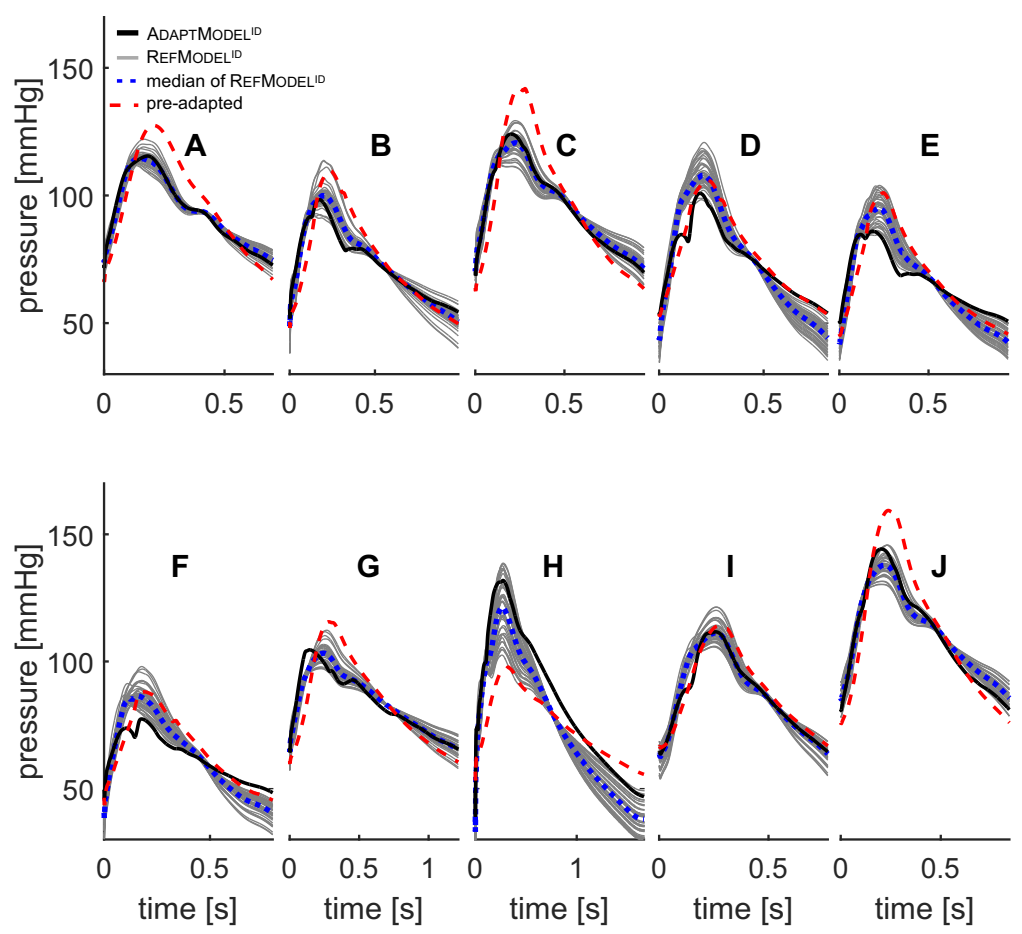

Figure 5.10: Aortic pressure waveforms for all ten (i.e. with IDs A to J) subjects. Pressure waveforms of the REFMoDEL are indicated by the grey curves. The median pressure waveforms of the reference waveforms are indicated by the blue dotted curves. The pressure waveforms generated by the pre-adapted model (i.e. with the vessels not adapted) are indicated by the red dashed curves.

\subsubsection{Sensitivity of haemodynamics and vessel geometry to adaptation model parameter variance}

Model response to changes in adaptation model parameters is shown in Table 5.3. Aortic systolic and diastolic pressure, as well as radial systolic- and diastolic pressure, were similarly sensitive to the target wall shear stress value of proximal arteries $(S=20.5 \%$ for sysyolic aortic pressure and systolic radial pressure and $S$ $=-29.8 \%$, and $-22.1 \%$ for diastolic aortic pressure and diastolic radial pressure, respectively). Diastolic radial pressure was sensitive to the target wall shear stress value of distal arteries $(S=29.4 \%)$. Output $c_{\text {pulse }}$ was also sensitive for the target wall stress value of distal arteries $(S=-19.5 \%)$.

Pulse pressure amplification ( $\left.\Delta p_{\text {amp }}\right)$, was most sensitive for target proximal wall shear stress $(S=-9.9 \%)$. Changes in the adaptation parameter $r_{\text {crit }}$ were of modest influence to waveform characteristics, with $\Delta p_{\text {amp }}$ as the most sensitive output metric to changes in $r_{\text {crit }}(S=5.2 \%)$. Model parameters governing radius adaptation significantly influenced $\epsilon_{h}$, reporting sensitivities between $-0.3 \%$ and 
Table 5.3: Sensitivity of selected haemodynamic characteristics and estimated geometries to adaptation parameters.

\begin{tabular}{lccccc}
\hline$y \downarrow, x \rightarrow$ & $\bar{\tau}_{\mathrm{w}, \text { target,prox }}$ & $\bar{\tau}_{\mathrm{w}, \text { target,dist }}$ & $r_{\text {crit }}$ & $\sigma_{\mathrm{f}, \text { target }}$ & $v_{\text {impact }}$ \\
\hline$p_{\text {s,aorta }}[\mathrm{mmHg}]$ & 20.5 & 29.4 & -2.2 & $\#$ & $\#$ \\
$p_{\text {d,aorta }}[\mathrm{mmHg}]$ & -29.8 & -44.5 & 3.1 & $\#$ & $\#$ \\
$p_{\text {s,radial }}[\mathrm{mmHg}]$ & 20.5 & 29.4 & -2.2 & $\#$ & $\#$ \\
$p_{\text {d,radial }}[\mathrm{mmHg}]$ & -22.1 & -45.0 & -5.0 & $\#$ & $\#$ \\
$c_{\text {pulse }}\left[\mathrm{m} \mathrm{s}^{-1}\right]$ & -17.7 & -19.5 & $\#$ & $\#$ & $\#$ \\
$\Delta p_{\text {amp }}[-]$ & -9.9 & -5.3 & 5.2 & $\#$ & $\#$ \\
$\epsilon_{r}[\mu \mathrm{m}]$ & -28.3 & 1.2 & -0.6 & $\#$ & $\#$ \\
$\epsilon_{h}[\mathrm{~mm}]$ & 6.3 & 6.6 & -0.3 & 65.0 & 37.6 \\
\hline
\end{tabular}

Values indicate sensitivity $S=(\Delta y / y) /(\Delta x / x) \cdot 100 \%$, of output metric $(y)$ to a $\pm 25 \%$ variation in adaptation model parameter $(x)$. \#: Sensitivity smaller than $0.05 \%$.

$6.6 \%$ (Table 5.3), in contrary to the negligible influence of model parameters governing wall thickness adaptation on $\epsilon_{r}$ (i.e. $|S|<0.05 \%$ ).

\subsection{Discussion}

The present study demonstrates the feasibility of predicting vessel geometry by incorporating vascular adaptation in a computational model of pulse wave propagation. This feasibility comes much at hand if confronted with sparse data sets on vessel geometry. Adaptation was based on the ability of vascular cells to sense perturbations in wall stress and wall shear stress and to mediate adaptation of vessel geometry to maintain mechanical homeostasis. A comparative evaluation between our adaptation approach and reference data demonstrated good agreement for geometry as well as pressure and flow waveforms along the modelled arterial domain.

Some aspects regarding our modelling assumptions warrant discussion. First, our model of radius adaptation is based on the hypothesis of constant mean wall shear stress regulation. The choice for mean wall shear stress was based on experimental work performed in dogs, studying the wall shear stress-radius relation under chronic increases in carotid artery flow. At six to eight months after the procedure, arterial remodelling caused lumen radius to increase, causing normalisation of carotid mean wall shear stress [20]. Similarly, Langille and 0'Donnell [24] reported that chronic decreases in carotid flow, imposed by ligation of the artery, resulted in reductions in carotid diameter. The second assumption relates to our model of wall thickness adaptation. We chose to incorporate an additional wall stress component, related to whole-body acceleration (Eq. 5.10). Our modelling assumption was based on findings from a previous numerical study [3]. Arts et al. [3] found it was crucial to incorporate the effect of whole-body acceleration to render physiological wall thickness values for both arteries and 
veins.

Using our proposed adaptation model, radius and wall thickness estimates were obtained independently from the generic vessel geometry, which was used as a takeoff point. To this end, we found negligible coefficients of variation for radius and wall thickness $\left(V_{r}\right.$ and $\left.V_{h} \leq 0.2 \%\right)$. This variation is well within the assumed measurement error of vascular ultrasound investigations [26].

For all subjects, limits of agreement between the model-estimated radius and reference data were in the order of magnitude of $2 \mathrm{~mm}$ for radius and $500 \mu \mathrm{m}$ for wall thickness. The reported reproducibility of the ultrasound-based measurement of dimensions lies between 0.2 and $0.3 \mathrm{~mm}$ [9, 37]. Furthermore, the theoretical ultrasound resolution for a $(7.5 \mathrm{MHz})$ clinical vascular probe is 0.2 $\mathrm{mm}$. Hence, we submit that our adaptation model yields reasonable estimations for detailed arterial trees like the one considered for this study.

In general, agreement between pressure and flow waveforms generated by the AdAPTModel and ReFModel was adequate for proximal arteries. For the example in Fig. 5.9, root-mean-square errors, $\epsilon_{p}$ and $\epsilon_{q}$, were 1.4 to $1.5 \mathrm{mmHg}$ and 0.3 to 7.6 $\mathrm{ml} \mathrm{s}^{-1}$, respectively. For the distally located arteries more pronounced differences in pressure and flow waveform shape were observed, especially during diastole (Fig. 5.9). However, the maximal difference in systolic, diastolic and mean pressure between adapted models and reference data was small. The predicted systolic blood pressure in the ascending aorta was within $1 \mathrm{mmHg}$ of its median reference value (Fig. 5.9). In the context of studies on non-invasive estimation of systolic central blood pressure, which have reported an agreement of $-1.3 \pm 3.2 \mathrm{mmHg}$ (bias $\pm \mathrm{SD}$, respectively), our method could yield clinically useful estimations of aortic blood pressure [35]. Similarly, estimated peak flow rate was in good agreement with reference values (difference in $q_{\text {sys }}$ within 10\%, Fig. 5.9). Furthermore, the root-mean-square errors $\left(\epsilon_{q}\right.$, Fig. 5.9) for flow rate were acceptable in the context of errors found in an in vitro study under steady flow conditions [12]. Peak flow rate predictions from PWP models could be used to define boundary conditions in e.g. aneurysm rupture risk assessment studies using subject-specific computational fluid dynamics [40]. In an earlier study, peak wall shear stress was found indicative of aneurysm rupture risk [8].

Differences in pressure and flow waveforms of the ADAPTMODEL and REFMODEL can have two causes: 1) the effect of errors between model-estimated geometries and reference geometries and 2) differences in three-element windkessel parameters between both models. For example, for peripheral vascular beds terminating proximal arterial branches, wave impedance was calculated using the ratio between inertance and compliance. Therefore, wave impedance depends on both radius and wall thickness, and could thus differ between the ADAPTMODEL and REFMODEL. We found negligible changes in our output of interest when evaluating the influence of choosing $Z_{0}$ equal to $10^{-2} \mathrm{~Pa} \mathrm{~s} \mathrm{~m}^{-3}$ for terminating distal arterial branches. In the current study, we modelled the periphery using the classical three-element windkessel model, assuming linear wave impedance, compliance and resistance (i.e. independent of pressure). In modelling adaptation of the peri- 
phery, our PWP-adaptation framework could benefit from alternative, potentially more appropriate models for the periphery by better capturing reflections at terminal branches of proximal arteries or by better capturing adaptation-induced changes in geometry by assuming non-linear $Z_{0}, R_{\mathrm{p}}$ and $C_{\text {art }}$, respectively $[3,30]$.

The local sensitivity analysis, quantifying how model output is influenced by changes in adaptation model parameters, clearly pointed out that adaptation model parameters should be determined for specific applications. For example, mean wall shear stress varies between healthy and diseased subjects, between arteries within the same subject, but also across species [32]. In choosing adequate adaptation model parameters, one could use values from earlier reports on measuring mean wall shear stress and wall stress in various species, in various types of patients as well as in different settings [6, 9, 27, 32]. Sensitivity analysis indicated that adaptation parameters for radius adaptation influence the outcome of wall thickness adaptation. This interdependency follows from our formulation of adaptation (Eqs. 5.9 and 5.14), and is based on physiology where wall stress also depends on vessel radius, which in turn is regulated by changes in flow rate [16]. As expected, adaptation model parameter $r_{\text {crit }}$ was of only modest influence to output metrics $(|S|<6 \%$, Table 5.3), since the smallest arteries in our model domain (i.e. the radial- and ulnar artery) have radii greater than $r_{\text {crit }}$.

\subsubsection{Previous work on modelling vascular adaptation}

Previous studies modelled vascular adaptation through stress-growth laws (e.g. Rachev [31]), or considered adaptation in extensive growth and remodelling frameworks (e.g. Humphrey and Rajagopal [17], Rodriguez et al. [34]). Such vascular adaptation models often assessed local arterial wall mechanics on the structural level through constitutive equations [17]. This allows for constitutive models to resemble a specific artery, for example, the basilar artery as in Valentin et al. [39]. Herein, physiologically realistic time-courses of elastin-collagen matrix turnover, remodelling and vascular smooth muscle cell tone in altered flow and intramural pressure situations were obtained [31, 39]. In contrast to the works as mentioned above, our current implementation of vascular adaptation does not take into account residual stresses, local stress distribution, and constituentspecific stresses. Instead, our implementation of vascular adaptation, relying on

only five model parameters (i.e. $\sigma_{\mathrm{f} \text {,target }}, \bar{\tau}_{\mathrm{w} \text {,target,prox }}, \bar{\tau}_{\mathrm{w} \text {,target,dist }}, r_{\text {crit }}$, and $v_{\text {impact }}$, respectively) can be considered a generalisation of these more extensive frameworks. Of note, despite our simplified physiological hypotheses in modelling vascular adaptation, we were able to adequately predict vessel geometry and haemodynamics.

\subsubsection{Limitations}

Comparative evaluation between measured vascular geometries and those estimated by our adaptation model was possible for a sufficiently large number of 
arterial segments (70 for radius and 20 for wall thickness, respectively). Analyses regarding pressure-flow waveforms required input of the geometries of all modelled arteries. To achieve this, we had to calculate subject-specific scaling factors to gain estimates for arteries that were not measured. Conclusions on the validity of model estimations, especially those containing assumptions regarding geometries of non-measured arteries, should be taken with some caution. Therefore, it should be emphasised that the present study does not validate our methodology, but serves to illustrate the usefulness of our PWP-adaptation framework when confronted with incomplete patient-specific data sets.

\subsection{Conclusions}

We incorporated an adaptation framework based on normalisation of wall stress and wall shear stress in blood vessels into a one-dimensional pulse wave propagation (PWP) model. Our PWP-adaptation framework calculates pressures and flows in blood vessels but does not require full parametrisation of the arterial domain. Instead, it considers adaptation processes to adequately predict arterial radius (bias $\pm 2 \mathrm{SD}$ of difference equal to $0.2 \pm 2.6 \mathrm{~mm})$ and wall thickness $(-140 \pm 557$ $\mu \mathrm{m})$. Such approach could benefit personalised modelling, notably in the case of missing values and sparse data sets as routinely occurs in clinical settings.

\section{Acknowledgements}

This study was funded by a Kootstra Talent Fellowship awarded to M.H.G. Heusinkveld by Maastricht University Medical Centre. R.J. Holtackers and B.P. Adriaans received funding from Stichting de Weijerhorst. 


\section{A1 Appendix: Data acquisition and processing steps}

\section{A1.1 Blood pressure and tonometry measurements}

Prior to measurements, subjects were rested at least 10 minutes in supine position during which oscillometric blood pressure measurements were obtained at the left upper arm (Omron 705IT, Omron Healthcare Europe, NL). After 10 minutes, tonometric waveforms were obtained at the brachial, radial and carotid artery (Sphygmocor, AtCor Medical, Sydney, AUS). The raw arterial tonometry waveforms were used to obtain calibrated local blood pressure waveforms using the method described by Kelly and Fitchett [21]. First, the tonometric waveform of the brachial artery (i.e. considered the reference artery) was calibrated using the oscillometric systolic and diastolic blood pressure values. Mean brachial pressure was then calculated as the numerical integral of the calibrated brachial waveform divided by pulse duration. Subsequently, the local pressures of the target arteries (i.e. carotid and radial) were obtained by calibrating tonometric waveforms using diastolic and mean brachial blood pressure assuming the difference between mean pressure and diastolic pressure to remain constant over the arterial tree [21].

\section{A1.2 Ultrasound imaging}

Ultrasound (US) measurements were performed immediately after tonometry measurements. Diameter measurements were performed at the brachial, radial, ulnar and carotid artery using an ESAOTE MyLab One (ESAOTE, Maastricht, NL) scanner equipped with a 4-13 MHz linear array probe (SL3323). Diameter waveforms were obtained by means of an RF-based echo wall-tracking tool (RFQAS, ESAOTE, Maastricht, NL). At the same locations, flow velocity waveforms were obtained by pulsed wave Doppler, with the sample volume set to cover the entire lumen. Carotid artery intima-media thickness was obtained using an automated software tool (RFQIMT, ESAOTE, Maastricht, NL).

Diameter waveforms and intima-media thickness values were obtained as averages of at least 4 heart beats. Radial and ulnar flow velocity curves were automatically traced by the US machine and mean flows were also obtained as averages over 4 to 6 heart beats, by multiplying diastolic lumen area with mean flow velocity.

\section{A1.3 MR imaging}

An overview of MRI sequence parameters is provided in Table A1.

Quantitative flow scans were analysed using CAAS MR Flow v1.2 (Pie Medical Imaging, Maastricht, NL). By indicating the vessel of interest, vessel contours were automatically detected on all cardiac frames. When necessary, manual adjustments were performed by a cardiologist who is trained and experienced in 
Table A1: MRI sequence parameters.

\begin{tabular}{lccc}
\hline & $\begin{array}{c}\text { 3D TFE } \\
\text { Aortic Arch }\end{array}$ & $\begin{array}{c}\text { 2D TFE } \\
\text { Quantitative flow }\end{array}$ & $\begin{array}{c}\text { 2D TSE BB } \\
\text { Vessel wall }\end{array}$ \\
\hline echo time [ms] & 1.0 & 2.2 & 60 \\
repetition time [ms] & 3.3 & 3.8 & 2 beats \\
flip angle [ ${ }^{\circ}$ ] & 20 & 10 & 90 \\
field of view [mm] & $320 \times 280 \times 120$ & $350 \times 300$ & $350 \times 350$ \\
slice thickness [mm] & - & 8 & 8 \\
acquired resolution [mm] & $2.0 \times 2.0 \times 3.0$ & $2.5 \times 2.5$ & $1.40 \times 1.75$ \\
reconstructed resolution [mm] & $1.0 \times 1.0 \times 1.5$ & $1.2 \times 1.2$ & $0.88 \times 0.88$ \\
\hline
\end{tabular}

analysing quantitative MR flow measurements (B.P.A.). After accepting all contours, the flow, maximum velocity, minimum velocity, mean velocity, and vessel area were calculated per cardiac phase and per flow-encoding direction. The net flow and net maximum velocity of each vessel was calculated using the data of all three flow-encoding directions. Black-blood vessel wall images were analysed using CAAS MR Viewer v4.3. 


\section{References}

[1] J. Alastruey, S. Moore, K. H. Parker, T. David, J. Peiró, and S. Sherwin. Reduced modelling of blood flow in the cerebral circulation: Coupling 1-D, 0-D and cerebral auto-regulation models. International Journal for Numerical Methods in Fluids, 56(8):1061-1067, 2008.

[2] F. C. Anderson and M. G. Pandy. Storage and utilization of elastic strain energy during jumping. Journal of Biomechanics, 26(12):1413-1427, 1993.

[3] T. Arts, K. Reesink, W. Kroon, and T. Delhaas. Simulation of adaptation of blood vessel geometry to flow and pressure: Implications for arterio-venous impedance. Mechanics Research Communications, 42:15-21, 2012.

[4] D. Bessems, M. C. M. Rutten, and F. N. van de Vosse. A wave propagation model of blood flow in large vessels using an approximate velocity profile function. Journal of Fluid Mechanics, 580: 145-168, 2007.

[5] E. Boileau, P. Nithiarasu, P. J. Blanco, L. O. Muller, F. E. Fossan, L. R. Hellevik, W. P. Donders, W. Huberts, M. Willemet, and J. Alastruey. A benchmark study of numerical schemes for onedimensional arterial blood flow modelling. International Journal for Numerical Methods in Biomedical Engineering, 31(10), 2015.

[6] C. G. Caro. The mechanics of the circulation. Cambridge University Press, 2012. ISBN 0521151775.

[7] A. Caroli, S. Manini, L. Antiga, K. Passera, B. Ene-Iordache, S. Rota, G. Remuzzi, A. Bode, J. Leermakers, F. N. van de Vosse, R. Vanholder, M. Malovrh, J. Tordoir, and A. Remuzzi. Validation of a patient-specific hemodynamic computational model for surgical planning of vascular access in hemodialysis patients. Kidney International, 84(6):1237-45, 2013.

[8] M. Castro, C. Putman, A. Radaelli, A. Frangi, and J. Cebral. Hemodynamics and rupture of terminal cerebral aneurysms. Academic Radiology, 16(10):1201-7, 2009.

[9] R. Dammers, J. H. M. Tordoir, J. M. M. Hameleers, P. J. E. H. M. Kitslaar, and A. P. G. Hoeks. Brachial artery shear stress is independent of gender or age and does not modify vessel wall mechanical properties. Ultrasound in Medicine \& Biology, 28(8):1015-1022, 2002.

[10] S. Epstein, M. Willemet, P. J. Chowienczyk, and J. Alastruey. Reducing the number of parameters in 1D arterial blood flow modeling: less is more for patient-specific simulations. American Journal of Physiology-Heart and Circulatory Physiology, 309(1):H222-H234, 2015.

[11] S. Glagov, C. Zarins, D. P. Giddens, and D. N. Ku. Hemodynamics and atherosclerosis. insights and perspectives gained from studies of human arteries. Archives of Pathologyathology \& Laboratory Medicine, 112(10):1018-1031, 1988.

[12] K. Hoyt, F. A. Hester, R. L. Bell, M. E. Lockhart, and M. L. Robbin. Accuracy of volumetric flow rate measurements: an in vitro study using modern ultrasound scanners. Journal of Ultrasound in Medicine, 28(11):1511-1518, 2009.

[13] W. Huberts, A. S. Bode, W. Kroon, R. N. Planken, J. H. M. Tordoir, F. N. van de Vosse, and E. M. H. Bosboom. A pulse wave propagation model to support decision-making in vascular access planning in the clinic. Medical Engineering \& Physics, 34(2):233-248, 2012. 
[14] W. Huberts, W. P. Donders, T. Delhaas, and F. N. van de Vosse. Applicability of the polynomial chaos expansion method for personalization of a cardiovascular pulse wave propagation model. International Journal for Numerical Methods in Biomedical Engineering, 31(7):e02720, 2015.

[15] T. J. R. Hughes and J. Lubliner. On the one-dimensional theory of blood flow in the larger vessels. Mathematical Biosciences, 18(1):161-170, 1973.

[16] J. D. Humphrey. Vascular adaptation and mechanical homeostasis at tissue, cellular, and subcellular levels. Cell Biochemistry and Biophysics, 50(2):53-78, 2008.

[17] J. D. Humphrey and K. R. Rajagopal. A constrained mixture model for growth and remodeling of soft tissues. Mathematical Models and Methods in Applied Sciences, 12(03):407-430, 2002.

[18] J. D. Humphrey, E. R. Dufresne, and M. A. Schwartz. Mechanotransduction and extracellular matrix homeostasis. Nature Reviews. Molecular Cell Biology, 15(12):802-12, 2014.

[19] G. N. Jager. Electrical model of the human arterial tree. PhD thesis, Utrecht University, 1965.

[20] A. Kamiya and T. Togawa. Adaptive regulation of wall shear stress to flow change in the canine carotid artery. American Journal of Physiology-Heart and Circulatory Physiology, 239(1):H14-H21, 1980.

[21] R. Kelly and D. Fitchett. Noninvasive determination of aortic input impedance and external left ventricular power output: a validation and repeatability study of a new technique. Journal of the American College of Cardiology, 20(4):952-963, 1992.

[22] V. A. Korshunov and B. C. Berk. Flow-induced vascular remodeling in the mouse a model for carotid intima-media thickening. Arteriosclerosis, Thrombosis, and Vascular Biology, 23(12): 2185-2191, 2003.

[23] W. Kroon, W. Huberts, E. M. H. Bosboom, and F. N. van de Vosse. A numerical method of reduced complexity for simulating vascular hemodynamics using coupled 0D lumped and 1D wave propagation models. Computational and Mathematical Methods in Medicine, (156094), 2012.

[24] B. L. Langille and F. O’Donnell. Reductions in arterial diameter produced by chronic decreases in blood flow are endothelium-dependent. Science, 231(4736):405-407, 1986.

[25] P. Laurent, P. Albaladejo, J. Blacher, A. Rudnichi, H. Smulyan, and M. E. Safar. Heart rate and pulse pressure amplification in hypertensive subjects. American Journal of Hypertension, 16(5): $363-370,2003$.

[26] C. A. D. Leguy, E. M. H. Bosboom, A. S. Z. Belloum, A. P. G. Hoeks, and F. N. van de Vosse. Global sensitivity analysis of a wave propagation model for arm arteries. Medical Engineering \& Physics, 33(8):1008-1016, 2011.

[27] T. Matsumoto and K. Hayashi. Stress and strain distribution in hypertensive and normotensive rat aorta considering residual strain. Journal of Biomechanical Engineering, 118(1):62-73, 1996.

[28] B. G. Miller, n. Gattone, V. H., J. M. Overhage, H. G. Bohlen, and A. P. Evan. Morphological evaluation of vascular smooth muscle cell: length and width from a single scanning electron micrograph of microvessels. Anatomical Record, 216(1):95-103, 1986.

[29] G. Mulder, A. C. Bogaerds, P. Rongen, and F. N. van de Vosse. The influence of contrast agent injection on physiological flow in the circle of willis. Medical Engineering and Physics, 33(2): 195-203, 2011.

[30] M. S. Olufsen. Structured tree outflow condition for blood flow in larger systemic arteries. American Journal of Physiology, 276(1 Pt 2):H257-68, 1999. 
[31] A. Rachev. A model of arterial adaptation to alterations in blood flow. Journal of Elasticity and the Physical Science of Solids, 61(1-3):83-111, 2000.

[32] R. S. Reneman and A. P. G. Hoeks. Wall shear stress as measured in vivo: consequences for the design of the arterial system. Medical \& Biological Engineering \& Computing, 46(5):499-507, 2008.

[33] P. Reymond, F. Merenda, F. Perren, D. Rufenacht, and N. Stergiopulos. Validation of a onedimensional model of the systemic arterial tree. Am J Physiol Heart Circ Physiol, 297(1):H208-22, 2009.

[34] E. K. Rodriguez, A. Hoger, and A. D. McCulloch. Stress-dependent finite growth in soft elastic tissues. Journal of Biomechanics, 27(4):455-467, 1994.

[35] J. E. Sharman, R. Lim, A. M. Qasem, J. S. Coombes, M. I. Burgess, J. Franco, P. Garrahy, I. B. Wilkinson, and T. H. Marwick. Validation of a generalized transfer function to noninvasively derive central blood pressure during exercise. Hypertension, 47(6):1203-1208, 2006.

[36] M. Stein. Large sample properties of simulations using latin hypercube sampling. Technometrics, 29(2):143-151, 1987.

[37] J. Steinbuch, A. P. G. Hoeks, E. Hermeling, M. T. B. Truijman, F. H. B. M. Schreuder, and W. H. Mess. Standard B-Mode ultrasound measures local carotid artery characteristics as reliably as radiofrequency phase tracking in symptomatic carotid artery patients. Ultrasound in Medicine \& Biology, 42(2):586-595, 2016.

[38] C. A. Taylor, C. P. Cheng, L. A. Espinosa, B. T. Tang, D. Parker, and R. J. Herfkens. In vivo quantification of blood flow and wall shear stress in the human abdominal aorta during lower limb exercise. Annals of Biomedical Engineering, 30(3):402-8, 2002.

[39] A. Valentin, L. Cardamone, S. Baek, and J. D. Humphrey. Complementary vasoactivity and matrix remodelling in arterial adaptations to altered flow and pressure. Journal of the Royal Society, Interface, 6(32):293-306, 2009.

[40] F. N. van de Vosse and N. Stergiopulos. Pulse wave propagation in the arterial tree. Annual Review of Fluid Mechanics, 43(1):467-499, 2011.

[41] S. M. Watanabe, P. J. Blanco, and R. A. Feijóo. Mathematical model of blood flow in an anatomically detailed arterial network of the arm. ESAIM: Mathematical Modelling and Numerical Analysis, 47 (4):961-985, 2013.

[42] N. Westerhof, F. Bosman, C. J. De Vries, and A. Noordergraaf. Analog studies of the human systemic arterial tree. Journal of Biomechanics, 2(2):121-143, 1969.

[43] H. Wolinsky. Long-term effects of hypertension on the rat aortic wall and their relation to concurrent aging changes: morphological and chemical studies. Circulation Research, 30(3): 301-309, 1972. 



\section{Non-invasive model-based estimation of left ventricular end-diastolic pressure and compliance}

The contents of this chapter are based on:

Maarten H.G. Heusinkveld, ${ }^{1}$ Tammo Delhaas, ${ }^{1}$ Katherine March, ${ }^{2}$ Justin E. Davies, ${ }^{3}$ Sayan Sen, ${ }^{3}$ Jamil Mayet, ${ }^{3}$ R. Arthur Bouwman, ${ }^{4,5}$ Joost Lumens, ${ }^{1}$ Alun D. Hughes, ${ }^{2}$ Koen D. Reesink ${ }^{1}$

Non-invasive model-based estimation of left ventricular end-diastolic pressure and compliance. (In preparation)

\footnotetext{
${ }^{1}$ Department of Biomedical Engineering, CARIM School for Cardiovascular Diseases, Maastricht University, Maastricht, The Netherlands.

${ }^{2}$ Department of Population Science and Experimental Medicine, University College London, London, United Kingdom.

${ }^{3}$ Department of Cardiology, Imperial College London, London, United Kingdom.

${ }^{4}$ Department of Anesthesiology, Catharina Hospital, Eindhoven, The Netherlands.

${ }^{5}$ Department of Electrical Engineering, Eindhoven University of Technology, Eindhoven, The Netherlands.
} 


\section{Uncertainty quantification and sensitivity analysis of an arterial wall mechanics model for evaluation of vascular drug therapies}

The contents of this chapter are based on:

Maarten H.G. Heusinkveld, ${ }^{1}$ Sjeng Quicken, ${ }^{1}$ Robert J. Holtackers, ${ }^{2}$ Wouter Huberts, ${ }^{1,3}$ Koen D. Reesink, ${ }^{1}$ Tammo Delhaas, ${ }^{1}$ Bart Spronck ${ }^{1,4}$ Uncertainty quantification and sensitivity analysis of an arterial wall mechanics model for evaluation of vascular drug therapies. Biomechanics and Modeling in Mechanobiology (2018) 17(2):55-69

\footnotetext{
${ }^{1}$ Department of Biomedical Engineering, CARIM School for Cardiovascular Diseases, Maastricht University, Maastricht, The Netherlands.

${ }^{2}$ Department of Radiology, CARIM School for Cardiovascular Diseases, Maastricht University, Maastricht, The Netherlands.

${ }^{3}$ Department of Biomedical Engineering, Eindhoven University of Technology, Eindhoven, The Netherlands.

${ }^{4}$ Department of Biomedical Sciences, Faculty of Medicine and Health Sciences, Macquarie University, Sydney, Australia.
} 


\subsection{Abstract}

Quantification of the uncertainty in constitutive model predictions describing arterial wall mechanics is vital towards non-invasive assessment of vascular drug therapies.

Therefore, we perform uncertainty quantification to determine uncertainty in mechanical characteristics describing the vessel wall response upon loading. Furthermore, a global variance-based sensitivity analysis is performed to pinpoint measurements that are most rewarding to be measured more precisely.

We used previously published carotid diameter-pressure and intima-media thickness (IMT) data (measured in triplicate), and Holzapfel-Gasser-Ogden models. A virtual data set containing 5000 diastolic and systolic diameter-pressure points, and IMT values was generated by adding measurement error to the average of the measured data. The model was fitted to single-exponential curves calculated from the data, obtaining distributions of constitutive parameters and constituent load bearing parameters.

Additionally, we 1) simulated vascular drug treatment to assess the relevance of model uncertainty and 2) evaluated how increasing the number of measurement repetitions influences model uncertainty. We found substantial uncertainty in constitutive parameters. Simulating vascular drug treatment predicted a 6percentage point reduction in collagen load bearing $\left(L_{\text {coll }}\right)$, approximately $50 \%$ of its uncertainty.

Sensitivity analysis indicated that the uncertainty in $L_{\text {coll }}$ was primarily caused by noise in distension and IMT measurements. Spread in $L_{\text {coll }}$ could be decreased by $50 \%$ when increasing the number of measurement repetitions from 3 to 10 .

Model uncertainty, notably that in $L_{\text {coll }}$, could conceal effects of vascular drug therapy. However, this uncertainty could be reduced by increasing the number of measurement repetitions of distension and wall thickness measurements used for model parametrisation. 


\subsection{Introduction}

RTERIAL stiffening is a major determinant of hypertension and vice versa

[19]. Treatment options for arterial stiffening can roughly be divided into two categories: 1) prescribing drugs that lower blood pressure and consequently reverse the arterial structural remodelling that occurs with hypertension, or 2) prescribing drugs that directly affect the arterial wall structure [42]. The second category includes cross-link breaking drugs that target the arterial collagen network $[20,48]$. These type of drugs aim to break down the advanced glycation end-products (AGE) that form cross-links between collagen molecules $[6,20,23]$. The desired mechanical effect of such drug therapies is to reverse pressure load bearing from a stiff, collagen dominated phenotype to a less stiff, elastin dominated phenotype; resulting in a decrease in material stiffness $[27,48]$.

In vivo assessment of the performance of vascular drugs has proven to be cumbersome [7]. Arterial stiffness is typically quantified by measuring carotid-femoral pulse wave velocity, or by local assessment of arterial distensibility [20,48]. A limitation of these indices is their blood pressure dependence, for which an incremental change in these indices could occur solely from a change in blood pressure [38]. In addition, arterial stiffness measurements as such do not yield insight into the effect of a drug at microstructural level, nor do they resolve whether the load-bearing phenotype is collagen or elastin dominated.

A potential solution to this problem lies in the use of computer models that simulate stress-strain behaviour of arteries using physical constitutive relations [15]. In such models, it might be possible to analyse the individual contribution of both elastin and collagen to the overall mechanical response of the vessel wall. Ex vivo studies on human carotid arteries, performed in the laboratory, reported good agreement between constitutive model computations and biaxial tensile tests $[35,36]$. If such models could be parametrised using non-invasive measurements in patients, they could be used to evaluate the mechanics of the vessel wall patient-specifically. Several studies have attempted to use in vivo data to parametrise constitutive models of the arterial wall, as reviewed in Spronck et al. [37]. Typically, diameter, pressure, and intima-media thickness measurements at the carotid artery are used to fit such models [37]. Generally, noise in those measurements will hamper patient-specific evaluation of arterial wall mechanics.

In this study, we aim to 1) assess how measurement uncertainty propagates into uncertainty of mechanical characteristics, estimated using a model of arterial wall mechanics and 2) pinpoint the measurements responsible for the largest spread in mechanical characteristics through sensitivity.

Uncertainty quantification and sensitivity analysis are considered indispensable tools to ensure credibility of computer model-based predictions [26]. We explicitly focus on two types of mechanical characteristics: 1) constitutive parameters, describing the mechanical properties of collagen and elastin; and 2) constituent load-bearing parameters, describing to which extent the blood pressure load is borne per constituent. The latter parameters are considered outcome parameters, obtained by evaluating the model using the best-fit set of constitutive 
parameters (Fig. 7.1, left panel). For these purposes, a large set of virtual pressure $(P)$, diameter $(D)$, and intima-media thickness (IMT) measurements will be generated by sampling the measurement distributions of previously published $P$, $D$ and IMT measurements in healthy volunteers [13].

For each $D-P$ sample, we will obtain continuous $D-P$ curves over the diastolicsystolic pressure range, using the single-exponential function described earlier $[24,11]$. Holzapfel-Gasser-Ogden constitutive models will be fitted to these data [15], and uncertainty quantification will be used to quantify the spread in the estimated mechanical characteristics, given the measurement noise (Fig. 7.1, left panel). A sensitivity analysis will be performed to determine how noise in the individual measured variables propagates into uncertainty in the model-predicted mechanical characteristics. The relevance of the model output uncertainty magnitude, which results from uncertainty in the model parameters, will be assessed by simulating the effect of AGE-breaker treatment on changes in collagen load bearing behaviour in our model (Fig. 7.1, right panel).

\subsection{Methods}

\subsubsection{Constitutive model definition}

To identify mechanical characteristics of the carotid artery wall, we used the Holzapfel-Gasser-Ogden constitutive model describing passive carotid artery wall mechanics [15]. This model was chosen on the one hand for its low number of parameters, and on the other hand for its feasibility in identifying mechanical characteristics when fitted to clinically obtained distensibility data [37]. Here, we will give a brief description of the model. For more details we refer to Appendix 7.A1.

In the model, the carotid artery is considered to be a thick-walled incompressible tubular structure composed of a mixture of two components: 1) elastin, assumed mechanically isotropic, and 2) collagen fibres, assumed mechanically orthotropic. Collagen was modelled by two families of fibres that are helically oriented at an angle of $+\beta_{0}$ and $-\beta_{0}$ with respect to the circumferential direction (Fig. 7.2). Elastin and collagen were assumed to operate in parallel. Furthermore, we assumed that there is only deformation along the principal axes, i.e. no shear. The mechanical response of the constituents can be described using a strain energy function (SEF). The SEFs of the constituents combined provide a relationship between stress and strain, and comprise a way of formulating constitutive behaviour of hyperelastic materials [18]. The SEF used for elastin $\left(W_{\text {elast }}\right)$ is given by

$$
W_{\text {elast }}=c_{\text {elast }}\left(I_{1}-3\right),
$$

with $I_{1}$ the first invariant of the Green-Lagrange strain tensor, given by $I_{1}=$ $\lambda_{r r}^{2}+\lambda_{\theta \theta}^{2}+\lambda_{z z}^{2}$, with $\lambda$ the stretch in the radial $(r)$ direction, circumferential $(\theta)$ direction, and axial (z) direction, respectively. The parameter $c_{\text {elast }}$ is a constitutive (stiffness) parameter for elastin. 


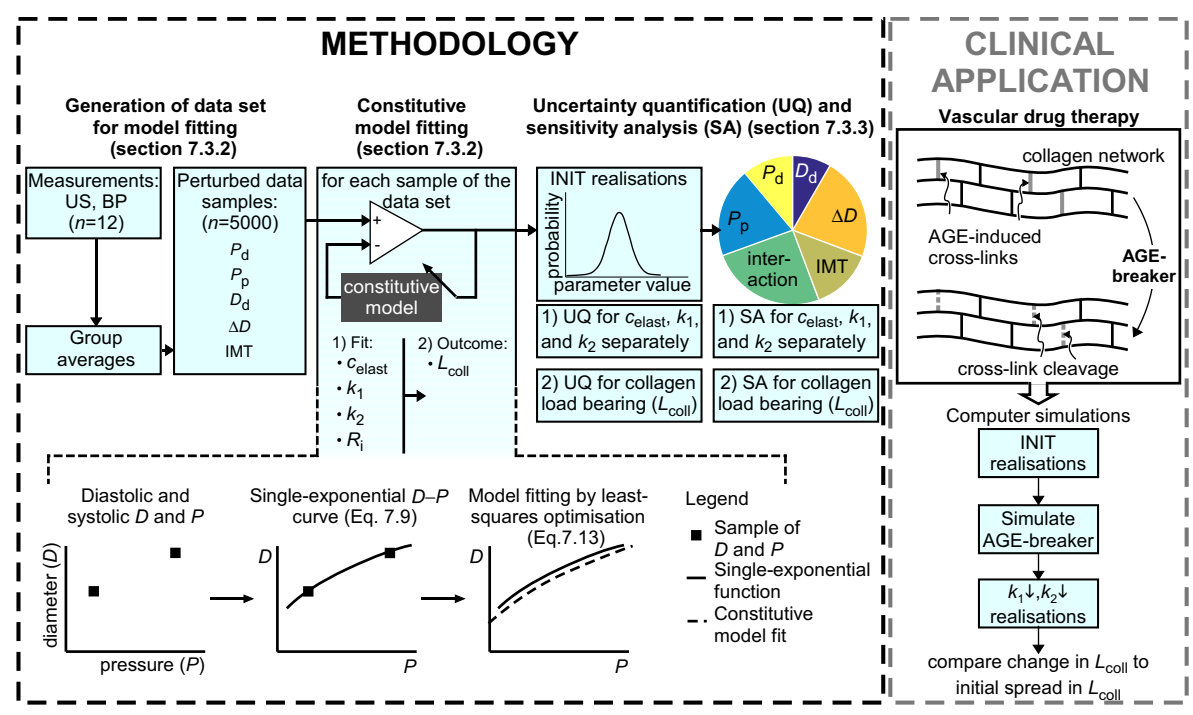

Figure 7.1: Outline of the current study, involving 1) uncertainty quantification (UQ) and sensitivity analysis (SA) of a constitutive model of arterial wall mechanics (methodology, left) and 2) assessment of the relevance of the model output uncertainty when evaluating the effects of vascular drug treatment (clinical application, right). Left: Group-averaged measured variables were obtained from a previous study, measuring twelve healthy subjects. The effect of measurement uncertainty on measured variables was included by perturbing group-averaged variables based on their measurement uncertainty. Within the margins of measurement uncertainty, we generated a data set composed of 5000 samples containing measured variables. Here, the measured variables are $D_{\mathrm{d}}$ : diastolic diameter; $\Delta D$ : distension; $P_{\mathrm{d}}$ and $P_{\mathrm{p}}$ : diastolic and pulse pressure, respectively; IMT: intima-media thickness. Holzapfel-Gasser-Ogden models were fitted to single-exponential diameter-pressure curves, determined for each sample of the generated data set. This yielded initial (INIT) realisations of mechanical characteristics, consisting of constitutive parameters and constituent load-bearing parameters, that were subsequently used for uncertainty quantification and sensitivity analysis. The constitutive parameters are $c_{\text {elast }}$ : elastin stiffness parameter; $k_{1}$ and $k_{2}$ : collagen stress-scaling and stress-curve shape parameters, respectively. The parameter $R_{\mathrm{i}}$ indicates the unstressed vessel inner radius and is an auxiliary model parameter. The load-bearing parameter $L_{\text {coll }}$ indicates the collagen load bearing as a percentage of the blood pressure (BP) load. Right: Assessing the relevance of uncertainty in load-bearing parameters by simulating advanced glycation end-product (AGE) breaker treatment using the model We simulated AGE-breaker treatment by reducing parameters $k_{1}$ and $k_{2}$ equally with respect to their INIT best-fit values.

The SEF used for collagen $\left(W_{\text {coll }}\right)$ is given by

$$
W_{\text {coll }}=\frac{k_{1}}{k_{2}}\left[\exp \left[k_{2}\left(\lambda_{\text {fibre }}^{2}-1\right)^{2}\right]-1\right] \text {, }
$$



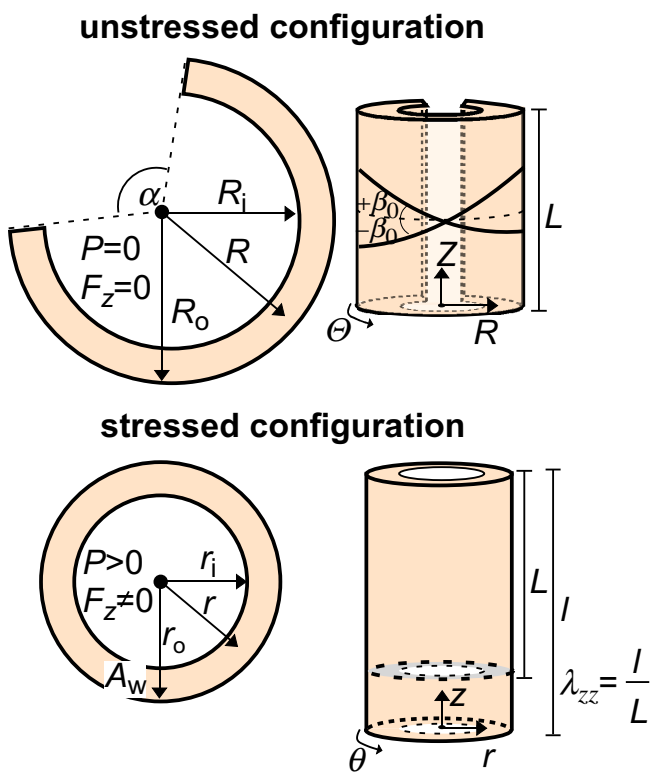

Figure 7.2: Overview of unstressed (top) and stressed (bottom) configurations of an artery. Top: $P$ : internal pressure, $F_{z}$ : reduced axial force, $R$ : unstressed radius, $R_{\mathrm{i}}$ : unstressed inner radius, $R_{\mathrm{o}}$ : unstressed outer radius, $\alpha$ : opening angle, $L$ : unstressed vessel length. In the unstressed configuration, we consider two families of helically orientated collagen fibres, with an angle $\pm \beta_{0}$ with respect to the circumferential direction. Bottom: $r$ : stressed radius, $r_{\mathrm{i}}$ : stressed inner radius, $r_{\mathrm{o}}$ : stressed outer radius, $A_{\mathrm{w}}$ : cross-sectional wall area in the stressed configuration, $l$ : stressed vessel length, $\lambda_{z z}$ : axial pre-stretch.

where $k_{1}$ as well as $k_{2}$ are constitutive parameters and $\lambda_{\text {fibre }}$ denotes fibre stretch, given by $\lambda_{\text {fibre }}=\sqrt{\cos ^{2}\left(\beta_{0}\right) \lambda_{\theta \theta}^{2}+\sin ^{2}\left(\beta_{0}\right) \lambda_{z z}^{2}}$. This collagen SEF should only contribute when the fibres are extended (while collagen fibres cannot sustain compression; Holzapfel et al. [15]). The positive luminal arterial pressure used in all our simulations ensures that such condition is always met.

For incompressible materials, the local stress-strain behaviour can be calculated using the derivatives of the SEFs:

$$
\sigma_{n}=-P_{\mathrm{h}}+\lambda_{n} \frac{\partial W_{\text {elast }}}{\partial \lambda_{n}}+\lambda_{n} \frac{\partial W_{\text {coll }}}{\partial \lambda_{n}},
$$

with $\sigma_{n}$ the Cauchy stress. The subscript $n$ is $r r$ for the radial direction, $\theta \theta$ for the circumferential direction and $z z$ for the axial direction. The pressure $P_{\mathrm{h}}$ is the local hydrostatic pressure within the wall.

In $W_{\text {elast }}$ and $W_{\text {coll }}$, the following parameters thus characterise the constitutive behaviour of elastin and collagen: 
- $c_{\text {elast }}$ : stiffness parameter of elastin, units of Pa.

- $k_{1}$ : stress scaling parameter of collagen, units of Pa.

- $k_{2}$ : collagen stress-curve shape parameter, dimensionless.

Elastin acts physiologically as the predominant load bearer for low pressure loads, whereas collagen, which is modelled as an unstressed fibrous material for low pressure, starts bearing load at higher pressures [14, 27, 32, 45]. The fitted parameters should result in a relation that mimics this behaviour. To quantify collagen load bearing, we estimated $L_{\text {coll }}$, being the pressure load borne by collagen as a percentage of the total blood pressure load (see Appendix 7.A1.4).

Based on literature reports on excised human and animal carotid arteries, we assumed that the artery is subjected to an initial axial pre-stretch $\left(\lambda_{z z}\right.$, Fig. 7.2) of 1.20 [37]. The helix angle of the two families of collagen fibres in our model was assumed to be $\pm 35.3^{\circ}$ in the unstressed configuration (Fig. 7.2) [1]. This angle was chosen from an analytical solution that for a thin-walled, fibre-reinforced tube results in a constant reduced axial force $\left(F_{z}\right)$ over the cardiac cycle $\left(\mathrm{d} F_{z} / \mathrm{d} P=0\right.$ $\left.\frac{\mathrm{N}}{\mathrm{Pa}}\right)[1,18,41,44,46]$. Here, $F_{z}$ is defined as the force applied in the axial direction additional to that generated by the pressure on the closed ends of the vessel [14]. Furthermore, in an earlier study fitting a thick-walled two-fibre material model to experimental data from coronary arteries, an optimal fibre angle of $36^{\circ}$ was found [43]. Cross-sectional wall area ( $A_{w}$, Fig. 7.2) was calculated from the diastolic outer diameter of the carotid $\left(D_{\mathrm{d}}\right)$ as well as IMT, using the relation given by

$$
A_{\mathrm{w}}=\pi\left(\frac{1}{4} D_{\mathrm{d}}^{2}-\left(\frac{1}{2} D_{\mathrm{d}}-\mathrm{IMT}\right)^{2}\right) .
$$

To map from a cut, stress-free configuration of an artery, to an unloaded intact configuration, to a loaded configuration, we define two additional parameters (Fig. $7.2,[16,18,37])$ : the opening angle $(\alpha)$ and the unstressed inner vessel radius $\left(R_{\mathrm{i}}\right)$. The value for $\alpha$ was taken from literature $\left(100^{\circ}\right.$, [37]). The parameter $R_{\mathrm{i}}$ was fitted using the constitutive model fitting routine (see below).

Residual stresses are accounted for by using the parameters $\lambda_{z z}, \alpha$ and $R_{\mathrm{i}}$ to define the stretches in the loaded configuration with respect to the stress-free configuration, as described in Appendix 7.A1. To obtain $D-P$ and $F_{\mathrm{z}}-P$ relations, the following boundary value problem was considered. Assuming axisymmetry and neglecting torsion, acceleration forces, body forces and axial extension, the relevant components of the radial momentum balance are given by

$$
\frac{\partial \sigma_{r r}}{\partial r}+\frac{\sigma_{r r}-\sigma_{\theta \theta}}{r}=0 .
$$

Applying the boundary conditions $\sigma_{r r}\left(r_{\mathrm{i}}\right)=-P$ and $\sigma_{r r}\left(r_{\mathrm{o}}\right)=0$, with $r_{\mathrm{i}}$ the vessel inner radius and $r_{\mathrm{o}}$ the vessel outer radius, the expression for lumen pressure is 
given by

$$
P=\int_{r_{i}}^{r_{o}} \frac{\sigma_{\theta \theta}-\sigma_{r r}}{r} \mathrm{~d} r .
$$

Reduced axial force is given by

$$
F_{z}=\pi \int_{r_{i}}^{r_{o}}\left(2 \sigma_{z z}-\sigma_{r r}-\sigma_{\theta \theta}\right) r \mathrm{~d} r .
$$

\subsubsection{Parametrisation}

\section{Clinical measurements}

The measurement protocol and data used in the present study are elaborated by Holtackers et al. [13]. Briefly, twelve apparently healthy volunteers (22 \pm 3 years, 6 male) were recruited. The study was approved by the medical ethics committee of Maastricht University Medical Centre (Maastricht, The Netherlands) and written consent was obtained from all participants prior to enrolment.

Ultrasound (US) B-mode recordings were performed at the right common carotid artery (CCA) in the anterolateral plane, and obtained in triplicate. Diastolic blood pressure $\left(P_{\mathrm{d}}\right)$ and pulse pressure $\left(P_{\mathrm{p}}\right)$ were measured three times during the US imaging protocol using an oscillometric device (Omron 705IT; Omron Healthcare Europe, Hoofddorp, The Netherlands).

The US recordings were analysed to determine right CCA cyclic distension (i.e. diastolic-systolic diameter change, $\Delta D)$ and diastolic diameter $\left(D_{\mathrm{d}}\right)$. Because the echo tracking tool used (RFQAS; Esaote, Maastricht, The Netherlands) utilises the media-adventitia echoes of near and far walls, we assumed the measured diameter signal over time to reflect the CCA outer diameter [37]. Furthermore, we obtained IMT at $P_{\mathrm{d}}$ using an automated software tool that reported good agreement with manual IMT methods [47].

\section{Generation of data set for constitutive modelling}

For the present analysis, we obtained an initial data set consisting of groupaveraged values for $P_{\mathrm{d}}, P_{\mathrm{p}}, D_{\mathrm{d}}, \Delta D$, and IMT (Fig. 7.1, left panel). Subsequently, uncertainties in the measured variables were accounted for by generating multiple samples within the uncertainty ranges of each measured metric. We assumed Gaussian distributed uncertainty domains for each measured variable $M_{i}$ in $\mathbf{M}$, given by

$$
M_{i}=\bar{M}_{i} \pm 1.96 \frac{\mathrm{SD}_{\text {intra }, M_{i}}}{\sqrt{N_{\text {rep }}}},
$$

see also Table 7.1.

Each sample consisted of a vector $\mathbf{M}$ containing the following variables: $\mathbf{M}=$ $\left[P_{\mathrm{d}}, P_{\mathrm{p}}, D_{\mathrm{d}}, \Delta D, \mathrm{IMT}\right]$. Here, $\bar{M}_{i}$ represents the average measured value of variable $M_{i}$ and $\mathrm{SD}_{\text {intra, } M_{i}}$ represents the corresponding intra-subject standard deviation 
Table 7.1: Overview of average values, intra-subject SDs and uncertainty domains per measured variable.

Data of twelve subjects

\begin{tabular}{llccc}
\hline Parameter & Unit & Mean & Intra-subject SD & Uncertainty domain \\
\hline$P_{\mathrm{d}}$ & $\mathrm{mmHg}$ & 72 & $3 \pm 0$ & {$[69 ; 75]$} \\
$P_{\mathrm{p}}$ & $\mathrm{mmHg}$ & 58 & $3 \pm 1$ & {$[54 ; 62]$} \\
$D_{\mathrm{d}}$ & $\mathrm{mm}$ & $6 \pm 37$ & $0 \pm 22$ & {$[6.12 ; 6.62]$} \\
$\Delta D$ & $\mathrm{~mm}$ & $0 \pm 789$ & $0 \pm 035$ & {$[0.750 ; 0.829]$} \\
$\mathrm{IMT}$ & $\mu \mathrm{m}$ & 539 & 40 & {$[494 ; 584]$} \\
\hline
\end{tabular}

Intima-media thickness (IMT) was obtained at $P_{\mathrm{d}}$. Intra-subject SD was determined using the method described in Bland and Altman [3]. The uncertainty domains of the measured variables were calculated using their respective mean and intra-subject SD values, see Eq. 7.8.

(i.e. indicating measurement error [3]). In our data set, measurements were performed in triplicate (i.e. $N_{\text {rep }}=3$ ) [13]. Samples were generated within the uncertainty domains of the measured variables, using Sobol's low discrepancy series, implemented in the MATLAB Statistics and Machine Learning Toolbox function sobolset (MATLAB R2015a; The MathWorks, Natick, MA, USA) [34]. This sampling method samples the uncertainty domains uniformly and was chosen as a "worst case scenario". To ensure adequate convergence of mechanical characteristics distributions, we generated 5000 samples. Systolic blood pressure $\left(P_{\mathrm{s}}\right)$ was calculated as $P_{\mathrm{s}}=P_{\mathrm{d}}+P_{\mathrm{p}}$; systolic diameter $\left(D_{\mathrm{s}}\right)$ was calculated as $D_{\mathrm{s}}=D_{\mathrm{d}}+\Delta D$. The distributions of $D_{\mathrm{d}}, D_{\mathrm{s}}, P_{\mathrm{d}}$, and $P_{\mathrm{s}}$ as well as IMT are shown in Fig. 7.3. The greater amount of scattering of systolic $D$ and $P$ data points compared to diastolic data points is caused by the fact that systolic blood pressure is defined as the sum of diastolic and pulse pressure.

As diastolic and pulse pressure were assumed to be independent, their sum (systolic blood pressure) will have a larger spread than diastolic blood pressure alone. The larger spread in systolic diameter than in diastolic diameter has the same origin, as systolic diameter is defined as the sum of diastolic diameter and distension.

For each sample in the data set, the following single-exponential function was parametrised to obtain continuous $D-P$ relations:

$$
P(D)=P_{\mathrm{d}} \exp \left[\gamma\left(\frac{D^{2}}{D_{\mathrm{d}}^{2}}-1\right)\right] .
$$

Here, $D$ is a continuous variable describing vessel diameter and $\gamma$ is a dimensionless non-linearity factor, which is calculated from systolic and diastolic blood 

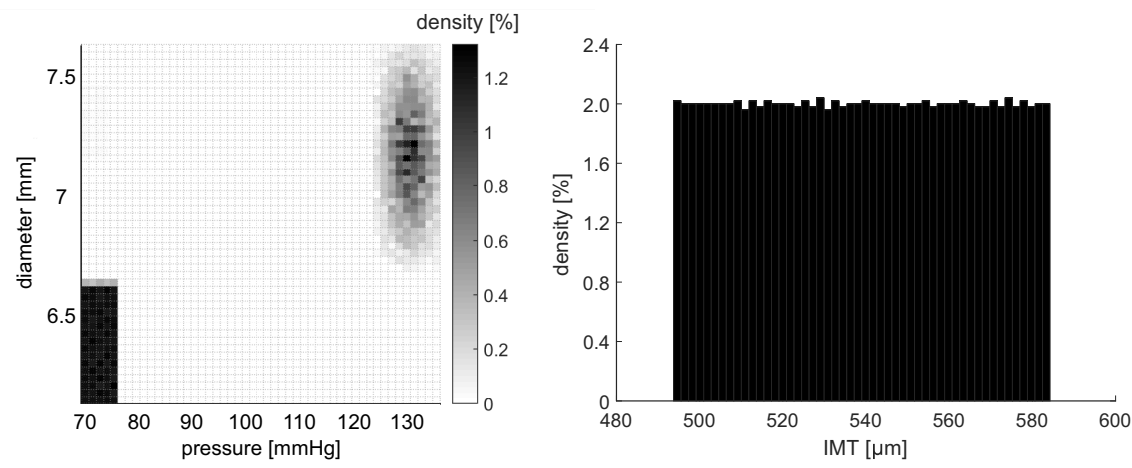

Figure 7.3: Density plots displaying the distributions of the 5000 computer-generated samples for diastolic and systolic blood pressure and diameter (left), as well as intima-media thickness (right). Density of diastolic pressure and diameter as well as intima-media thickness samples is high. The greater amount of scattering of systolic diameter and pressure samples arises from the fact that systolic blood pressure is defined as the sum of diastolic and pulse pressure, assumed independent. Similarly systolic diameter is defined as the sum of diastolic diameter and distension, also assumed independent.

pressures and diameters as described by Meinders and Hoeks [24]:

$$
\gamma=\frac{\log \left(\frac{P_{\mathrm{s}}}{P_{\mathrm{d}}}\right)}{\frac{D_{\mathrm{s}}^{2}}{D_{\mathrm{d}}^{2}}-1} .
$$

\section{Model fitting procedure}

The constitutive model was fitted to the single-exponential curve, obtained for each sample $\mathbf{M}$ of the data set. Model fitting was performed by variation of model parameters $c_{\text {elast }}, k_{1}, k_{2}$, and $R_{\mathrm{i}}$ (Fig. 7.1, left panel). Lower and upper bounds of all fitted parameters are given in Table 7.2.

Table 7.2: Complete overview of lower and upper parameter bounds used for fitting the diameter-pressure data.

\begin{tabular}{llcc}
\hline Parameter & Unit & Lower bound & Upper bound \\
\hline$c_{\text {elast }}$ & $\mathrm{kPa}$ & 1 & 400 \\
$k_{1}$ & $\mathrm{kPa}$ & $0.1 \cdot 10^{-3}$ & 400 \\
$k_{2}$ & - & 0 & 100 \\
$R_{\mathrm{i}}$ & $\mathrm{m}$ & $0.5 \cdot 10^{-3}$ & $10 \cdot 10^{-3}$ \\
\hline
\end{tabular}

For each sample in the data set, we assumed the single-exponential curve to be valid within the range $P \in\left\{P_{\mathrm{d} \text {,sample }}-15 \mathrm{mmHg}, \quad P_{\mathrm{s} \text {,sample }}+15 \mathrm{mmHg}\right\}$ 
$[11,24]$. In Fig. 7.3 (left), the distribution of $P_{\text {d,sample }}$ vs. $P_{\text {s,sample }}$ is displayed.

Fitting was performed using the trust-region reflective algorithm [25], implemented in the MATLAB Optimization Toolbox function lsqnonlin and was initiated from 10 random start points in the parameter space using the MATLAB Global Optimization Toolbox function MultiStart. The same 10 start points were used for fitting all samples. Throughout model fitting, we aimed to minimise the sum of squared differences between measured pressure from the single-exponential curve $P_{j}$ and modelled $P_{\text {mod, } j}$ :

$$
\epsilon_{P}=\frac{1}{n_{P}} \frac{1}{P_{\mathrm{p}}^{2}} \sum_{j=1}^{n_{P}}\left(P_{j}-P_{\mathrm{mod}, j}\right)^{2},
$$

where, for each sample, $n_{P}$ is the number of fitting points and $P_{\mathrm{p}}$ is the pulse pressure.

As a physiological constraint, $F_{z}$ was forced to remain constant at a target value defined $F_{z \text {,target }}$, with varying pressure. This assumption was based on experimental work performed by Van Loon [44] and Weizsäcker et al. [46], observing axial force to be nearly insensitive to changes in pressure for arteries inflated at their in vivo axial pre-stretch. We enforced this constraint by minimising the following expression:

$$
\epsilon_{F_{z}}=\frac{1}{n_{P}} \frac{1}{F_{z, \text { target }}^{2}} \sum_{j=1}^{n_{P}}\left(F_{z, j}-F_{z, \text { target }}\right)^{2} \text {, }
$$

where $F_{z \text {,target }}$ was assumed to be equal to $0.5 \mathrm{~N}$. This value was based on a study by Patel et al. [28] in excised, vertically suspended canine arteries that were extended by hanging weights from the bottom end of the artery. For the carotid artery to restore its in vivo length, a weight of $54 \mathrm{~g}$ was required, corresponding to an $F_{z}$ of $0.5 \mathrm{~N}$. The two weighted errors $\epsilon_{P}$ and $\epsilon_{F_{z}}$ are combined into the weighted total sum of squares $\left(\epsilon_{\mathrm{T}}\right)$ :

$$
\epsilon_{\mathrm{T}}=w_{P} \epsilon_{P}+w_{F_{z}} \epsilon_{F_{z}},
$$

where $w_{P}$ and $w_{F_{z}}$ are non-dimensional weighting factors. Here, we chose $w_{P}=10$ and $w_{F_{z}}=1$. The $\epsilon_{P}$ term was given a higher importance than the $\epsilon_{F_{z}}$ term, because of absence of $F_{z}$ measurements in our study and uncertainty in the assumed target value for $F_{z}$. The fitting error describing goodness-of-fit is expressed as a normalised root mean square error $\left(E_{\mathrm{RMS}, P}\right.$ and $\left.E_{\mathrm{RMS}, F_{z}}\right)$ :

$$
\begin{aligned}
E_{\mathrm{RMS}, P} & =100 \% \cdot \sqrt{\epsilon_{P}}, \quad \text { and } \\
E_{\mathrm{RMS}, F_{z}} & =100 \% \cdot \sqrt{\epsilon_{F_{z}}} .
\end{aligned}
$$




\subsubsection{Simulations and analysis}

\section{Initial constitutive parameter estimation}

The constitutive model was fitted to all 5000 samples of the generated data set using the procedure explained in the previous section. This yielded 5000 initial constitutive model realisations (i.e. termed INIT).

\section{Uncertainty quantification and sensitivity analysis}

All constitutive model realisations together yield insight in the distribution of the mechanical characteristics that results from the presence of measurement uncertainty. This distribution of mechanical characteristics was therefore used to quantify the uncertainty in constitutive parameters (i.e. the fitted parameters $c_{\text {elast }}, k_{1}$, and $k_{2}$ ), as well as collagen load bearing parameters, (i.e. the outcome parameter $L_{\text {coll }}$ at various blood pressure levels, Fig. 7.1, left panel). We used kernel density estimation (KDE) to visualise the distributions of constitutive parameters and load bearing parameters. KDE estimates the probability density function, which in this context implies the probability density of finding a certain value of a constitutive parameter or load bearing value [33]. Furthermore, we quantified spread in parameters using the median and the $25^{\text {th }}$ to $75^{\text {th }}$ percentile confidence interval $\left(\mathrm{PCI}_{25 \rightarrow 75}\right)$. Calculating the $\mathrm{PCI}_{25 \rightarrow 75}$ comes at hand when assessing spread of skewed distributions.

Sensitivity analysis (SA) was subsequently used to apportion uncertainty in the model-predicted mechanical characteristics to uncertainty in specific measured variables, or their interactions [17]. A global variance-based SA was performed using regression-based adaptive generalised polynomial chaos expansion (agPCE), as detailed in Quicken et al. [29]. The agPCE method captures the relation between mechanical characteristics $\left(X_{i}\right)$ and measured variables $\left(M_{i}\right)$ by means of an adaptively constructed finite polynomial expansion $f_{\text {agPCE }}$ :

$$
X_{i}=f^{X_{i}}(\mathbf{M}) \approx f_{\mathrm{agPCE}}^{X_{i}}(\mathbf{M}) .
$$

Here, $\mathbf{X}$ contains the mechanical characteristics, i.e. $\mathbf{X}=\left[c_{\text {elast }}, k_{1}, k_{2}, L_{\text {coll }}\right]$. Furthermore, $\mathbf{M}=\left[M_{1}, M_{2}, \ldots, M_{N_{\text {vars }}}\right]=\left[P_{\mathrm{d}}, P_{\mathrm{p}}, D_{\mathrm{d}}, \Delta D\right.$, IMT $]$, and $N_{\text {vars }}$ is the number of measured variables. Such a polynomial expansion provides a meta-model of the mechanical characteristics estimation method. After constructing the meta-model, the value of the leave-one-out cross-validation coefficient $\left(Q^{2}\right)$ was computed. Coefficient $Q^{2}$, ranging between a value of 0 and 1 is a quality measure of the meta-model, indicating its predictive properties [40]. Throughout this study we assumed $Q^{2}>0.99$ to indicate an appropriate meta-model.

From the meta-model, the variance of a mechanical characteristic (a measure of its uncertainty) can be computed. The following sensitivity metrics were computed:

- Main sensitivity indices. The main sensitivity index $\left(S_{\mathrm{i}}\right)$ of measured vari- 
able $M_{i}$ represents the expected reduction in uncertainty of the mechanical characteristic if $M_{i}$ were known exactly. Assessment of $S_{\mathrm{i}}$ determines which measured variables are most rewarding to be measured more accurately to reduce model output uncertainty (i.e. parameter prioritisation) [31].

- Total sensitivity indices. The total sensitivity index $\left(S_{\mathrm{T}}\right)$ of $M_{i}$ represents the expected uncertainty in the mechanical characteristic that would remain if all other measured variables except $M_{i}$ were known exactly. Assessment of $S_{\mathrm{T}}$ determines which measured variables could potentially be fixed within their uncertainty domain (i.e. parameter fixing) [31].

The contribution of each measured variable to total uncertainty in estimating mechanical characteristics can be illustrated as the segments of a disc (Fig. 7.1). Total uncertainty may be apportioned to single measured variables but, may also arise from interaction between measured variables (Fig. 7.1). Moreover, significant interaction effects are indicated by large differences between total sensitivity indices and main sensitivity indices [39].

\section{Simulating the effect of AGE-breaker vascular drugs}

AGE-breaker treatment was simulated using the INIT realisations as take-off points (Fig. 7.1, right panel). We explicitly assumed that a reduction in cross-link density can be represented in the model by reducing parameters $k_{1}$ and $k_{2}$. The rationale of reducing $k_{1}$ and $k_{2}$ is based on previous work measuring the stressstrain response of collagen tissue at multiple levels of cross-linking [8,21]. In their work it was observed that a decrease in cross-link density results in 1) a decrease in fibre stiffness at low amounts of strain and 2) a decrease in the non-linearity of the fibre stress-strain response $[8,21]$. In our analysis, $k_{1}$ and $k_{2}$ were equally reduced by $40 \%$ of their initial best-fit value yielding the $k_{1} \downarrow, k_{2} \downarrow$ realisations (Fig. 7.1, right panel). All other constitutive model parameters were assumed to remain unchanged.

\subsection{Results}

\subsubsection{Representative example of a fitted constitutive model}

A representative example of a model-based $D-P$ and $F_{z}-P$ relationship, obtained by model-fitting a sample within the virtual data set is depicted in Fig. 7.4. The bestfit constitutive parameters for this representative sample were $c_{\text {elast }}=40.1 \mathrm{kPa}$, $k_{1}=6.9 \mathrm{kPa}$ and $k_{2}=8.5$. Collagen load bearing $\left(L_{\text {coll }}\right)$ increased monotonically with blood pressure from $0.6 \%$ at diastolic blood pressure $\left(P_{\mathrm{d}}\right)$, to $10.2 \%$ at mean arterial pressure $\left(P_{\mathrm{m}}\right)$, up to $25.0 \%$ at systolic blood pressure $\left(P_{\mathrm{s}}\right)$, respectively. The slope of the plotted curves $(\mathrm{d} D / \mathrm{d} P)$ is a measure of vascular compliance. Low vascular compliance corresponds to high vascular stiffness and vice versa. 

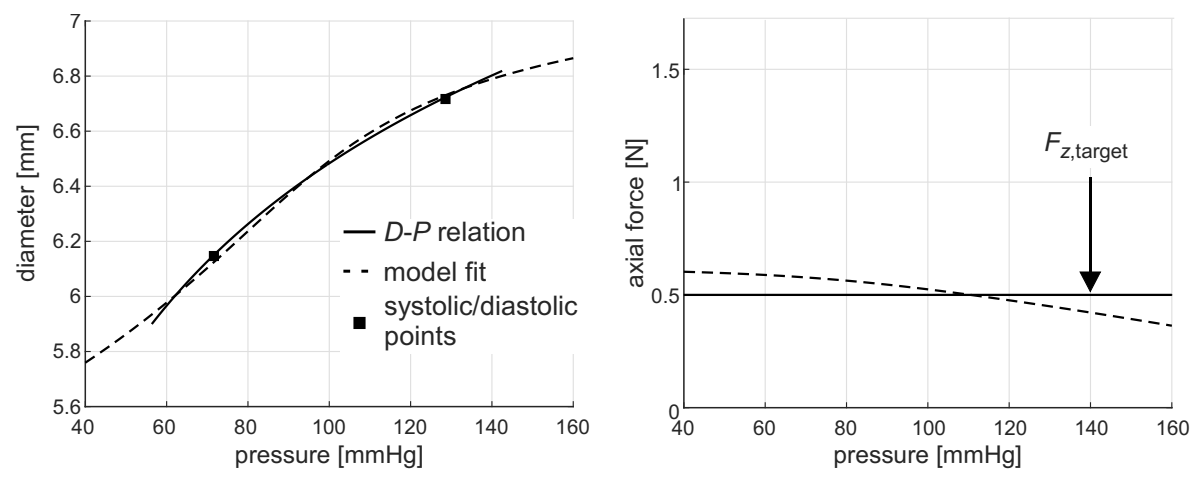

Figure 7.4: Representative example of the model-predicted diameter-pressure $(D-P)$ curve and the reduced axial force-pressure $\left(F_{z}-P\right)$ curve (dashed lines) as well as the group-averaged data fitted upon (solid lines). The difference between measured and model-predicted pressure was minimised over the pressure fitting range (i.e. $P_{\mathrm{d}}-15 \mathrm{mmHg}$ to $P_{\mathrm{s}}+15 \mathrm{mmHg}$ ), whereas reduced axial force was fitted to an assumed target value of $0.5 \mathrm{~N}\left(F_{z \text {,target }}\right.$, respectively). Values of fitted parameters were $c_{\text {elast }}=40.1 \mathrm{kPa}, k_{1}=6.9 \mathrm{kPa}$ and $k_{2}=8.5$. Fit errors, describing the goodness-of-fit were $E_{\mathrm{RMS}, P}=0.020 \%$ and $E_{\mathrm{RMS}, F_{z}}=16 \%$, respectively.

The model fit (dashed lines, Fig. 7.4) shows sigmoidal $D-P$ behaviour over a 40 to $160 \mathrm{mmHg}$ pressure range, suggesting low compliance for the lower part of pressure range followed by higher compliance in the physiological pressure range and again lower compliance for the upper part of the pressure range. Furthermore, there is some deviation between the model-based $F_{z}-P$ curve and the assumed (pressure-independent) target value of $0.5 \mathrm{~N}$ (Fig. 7.4).

\subsubsection{Uncertainty quantification and sensitivity analysis}

The distributions of the fitted constitutive parameters are shown in Fig. 7.5.
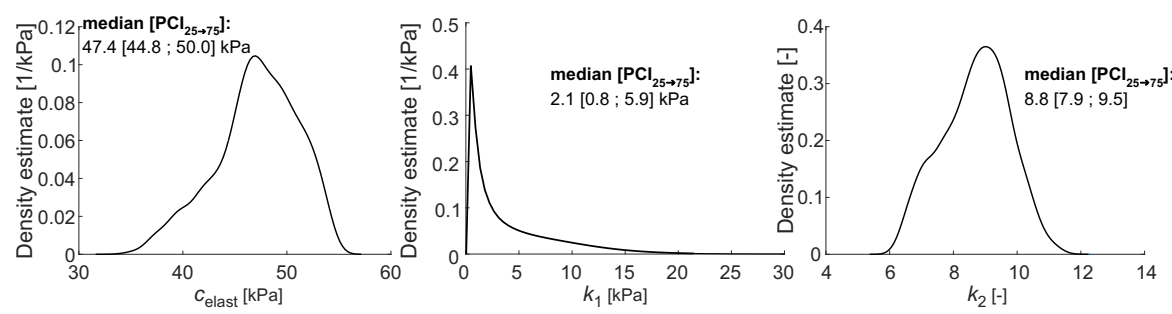

Figure 7.5: Kernel density estimates (KDE) describing the distributions of fitted constitutive parameters $\left(c_{\text {elast }}, k_{1}\right.$, and $\left.k_{2}\right)$. Distributions of parameters were quantified using the median and the $25^{\text {th }}$ to $75^{\text {th }}$ percentile confidence interval $\left(\mathrm{PCI}_{25 \rightarrow 75}\right)$, respectively.

Best-fit parameter values for elastin stiffness $\left(c_{\text {elast }}\right)$ were $47.4[44.8 ; 50.0] \mathrm{kPa}$ 
(median $\left[\mathrm{PCI}_{25 \rightarrow 75}\right]$ ). For collagen parameters, best-fit parameter values were 2.1 $[0.8 ; 5.9]) \mathrm{kPa}$ for $k_{1}$, and 8.8 [7.9;9.5]) for $k_{2}$, respectively.

In Fig. 7.6, distributions of collagen load bearing parameters $\left(L_{\text {coll }}\right)$ are given at three blood pressure levels $\left(P_{\mathrm{d}}, P_{\mathrm{m}}\right.$, and $P_{\mathrm{s}}$, respectively).
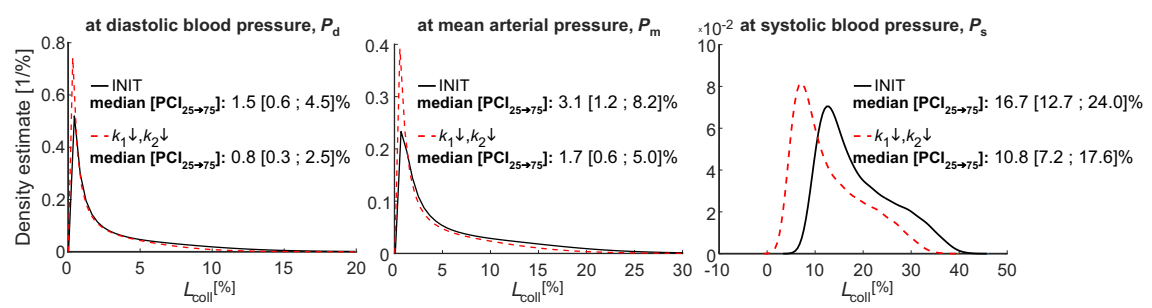

Figure 7.6: Kernel density estimates (KDEs) describing the distribution of collagen load bearing characteristic $\left(L_{\text {coll }}\right)$. Black line: KDE for initial constitutive model realisations (INIT). Red dashed line: KDEs for model with decreased $k_{1}$ and $k_{2}\left(k_{1} \downarrow, k_{2} \downarrow\right)$. KDEs were calculated at three blood pressure levels; diastolic blood pressure $\left(P_{\mathrm{d}}\right)$, mean arterial pressure $\left(P_{\mathrm{m}}\right)$ and systolic blood pressure $\left(P_{s}\right)$. Distributions of parameters were quantified using the median and the $25^{\text {th }}$ to $75^{\text {th }}$ percentile confidence interval $\left(\mathrm{PCI}_{25 \rightarrow 75}\right)$, respectively.

Note that $L_{\text {coll }}=0 \%$ indicates that blood pressure load is fully borne by elastin, whereas $L_{\text {coll }}=100 \%$ indicates that blood pressure load is fully borne by collagen. For the initial constitutive model realisations (INIT), we found $L_{\text {coll }}$ equal to $1.5 \%$ at $P_{\mathrm{d}}, 3.1 \%$ at $P_{\mathrm{m}}$, and $16.7 \%$ at $P_{\mathrm{s}}$ (medians, respectively). As shown in Fig. 7.6, the $25^{\text {th }}$ to $75^{\text {th }}$ percentile confidence interval for $L_{\text {coll }}$ at $P_{\mathrm{d}}$ as well as $P_{\mathrm{s}}$ were large $([0.6 ; 4.5] \%$ and $[12.7 ; 24.0] \%$, respectively), indicating large uncertainty in model predictions of constituent load bearing.

A full overview of main and total sensitivity indices is given in Tables 7.3 and 7.4 .

Table 7.3: Main $\left(S_{\mathrm{i}}\right)$ and total $\left(S_{\mathrm{T}}\right)$ sensitivity indices for constitutive parameters $c_{\text {elast }}, k_{1}$ and $k_{2}$.

\begin{tabular}{llccccccc} 
& & \multicolumn{2}{c}{ Constitutive parameter: } & & \multicolumn{2}{c}{$k_{2}$} \\
\hline Measured variable & Symbol & $S_{\mathrm{i}}$ & $S_{\mathrm{T}}$ & $S_{\mathrm{i}}$ & $S_{\mathrm{T}}$ & $S_{\mathrm{i}}$ & $S_{\mathrm{T}}$ \\
\hline Diastolic blood pressure & $P_{\mathrm{d}}$ & 0.012 & 0.014 & 0.00060 & 0.0011 & 0.052 & 0.056 \\
Pulse pressure & $P_{\mathrm{p}}$ & 0.0018 & 0.021 & 0.027 & 0.041 & 0.10 & $\mathbf{0 . 1 2}$ \\
Diastolic diameter & $D_{\mathrm{d}}$ & 0.0060 & 0.036 & 0.033 & 0.047 & $\mathbf{0 . 2 9}$ & $\mathbf{0 . 3 2}$ \\
Distension & $\Delta D$ & $\mathbf{0 . 6 2}$ & $\mathbf{0 . 6 7}$ & $\mathbf{0 . 9 0}$ & $\mathbf{0 . 9 3}$ & $\mathbf{0 . 5 0}$ & $\mathbf{0 . 5 4}$ \\
Intima-media thickness & $\mathrm{IMT}$ & $\mathbf{0 . 3 1}$ & $\mathbf{0 . 3 1}$ & 0.0083 & 0.015 & 0.00083 & 0.00083 \\
\hline
\end{tabular}

Sensitivity indices larger than 0.10 are indicated in bold. The coefficient $Q^{2}$, indicating the accuracy of the meta-model, was $0.997,0.998$ and 0.998 for $c_{\text {elast }}, k_{1}$ and $k_{2}$, respectively

In general, distension and IMT were most rewarding to be measured more reliably. For the constitutive parameters $c_{\text {elast }}$ and $k_{1}$, this is illustrated by main 
Table 7.4: Main $\left(S_{\mathrm{i}}\right)$ and total $\left(S_{\mathrm{T}}\right)$ sensitivity indices for collagen load bearing parameters $\left(L_{\text {coll }}\right)$ at three blood pressure levels; diastolic- mean- and systolic blood pressure (i.e. $P_{\mathrm{d}}, P_{\mathrm{m}}$ and $P_{\mathrm{s}}$, respectively.)

\begin{tabular}{llccccccc} 
& & \multicolumn{2}{c}{ Load bearing parameter: } & & \multicolumn{2}{c}{$L_{\text {coll }}$ at $P_{\mathrm{s}}$} \\
\hline Measured variable & Symbol & $S_{\mathrm{i}}$ & $S_{\mathrm{T}}$ & $S_{\mathrm{i}}$ & $S_{\mathrm{T}}$ & $S_{\mathrm{i}}$ & $S_{\mathrm{T}}$ \\
\hline Diastolic blood pressure & $P_{\mathrm{d}}$ & 0.00043 & 0.00098 & 0.00073 & 0.0016 & 0.0019 & 0.0027 \\
Pulse pressure & $P_{\mathrm{p}}$ & 0.021 & 0.035 & 0.023 & 0.035 & 0.052 & 0.054 \\
Diastolic diameter & $D_{\mathrm{d}}$ & 0.036 & 0.058 & 0.041 & 0.059 & 0.064 & 0.067 \\
Distension & $\Delta D$ & $\mathbf{0 . 9 0}$ & $\mathbf{0 . 9 4}$ & $\mathbf{0 . 9 0}$ & $\mathbf{0 . 9 3}$ & $\mathbf{0 . 8 7}$ & $\mathbf{0 . 8 8}$ \\
Intima-media thickness & $\mathrm{IMT}$ & 0.0028 & 0.0049 & 0.0031 & 0.0046 & 0.0031 & 0.0048 \\
\hline
\end{tabular}

Sensitivity indices larger than 0.10 are indicated in bold. The coefficient $Q^{2}$, indicating the accuracy of the meta-model, was $0.993,0.995$ and 0.999 for diastolic $\left(P_{\mathrm{d}}\right)$, mean $\left(P_{\mathrm{m}}\right)$ and systolic blood pressure $\left(P_{\mathrm{s}}\right)$, respectively

sensitivity indices $\left(S_{\mathrm{i}}\right)$ between 0.62 and 0.90 for distension and 0.31 for IMT (Table 7.3).

For collagen parameter $k_{2}$, distension and diastolic diameter are most influential (Table 7.3). Moreover, pulse pressure has some influence, indicated by an $S_{\mathrm{i}}$ of 0.10 (Table 7.3). For collagen load bearing $\left(L_{\text {coll }}\right)$, distension was the most important measured variable, indicated by $S_{\mathrm{i}}$ between 0.87 and 0.90 (Table 7.4). The main sensitivity indices of the other measured variables were smaller than 0.06, indicating low influence (Table 7.4).

Reducing uncertainty of blood pressure measurements appears of negligible importance in reducing uncertainty in estimating $c_{\text {elast }}, k_{1}$, and $L_{\text {coll }}$, indicated by an $S_{\mathrm{i}}<0.06$ (Table 7.3). Moreover, total sensitivity indices $\left(S_{\mathrm{T}}\right)$ for diastolic blood pressure and pulse pressure were smaller than 0.12 , suggesting these variables could be fixed in their uncertainty domain.

For all measured variables, differences between main and total sensitivity indices were minor, i.e. $S_{\mathrm{T}}-S_{\mathrm{i}}$ was smaller than 0.10 (Tables 7.3 and 7.4). This indicates that the contribution of interaction terms between measured variables to the total variance was negligible [31].

\subsubsection{Model-based assessment of vascular drug therapies}

Fig. 7.7 shows the effect of AGE-breaker treatment (simulated by $k_{1} \downarrow, k_{2} \downarrow$ ) on the model-predicted $D-P$ curve, as well as on area compliance $\left(C_{A}\right)$.

Here, the average $D-P$ curves, originating on the one hand from the initial best-fit constitutive parameters (INIT, black line), and on the other hand following reduction of $k_{1}$ and $k_{2}\left(k_{1} \downarrow, k_{2} \downarrow\right.$, red dashed line) are shown. Moreover, $C_{A}$ is shown in the right panel for all INIT realisations (black circles), and the $k_{1} \downarrow, k_{2} \downarrow$ realisations (red circles). Area compliance was calculated using $C_{A}=\left(A_{\mathrm{s}}-A_{\mathrm{d}}\right) /\left(P_{\mathrm{s}}-P_{\mathrm{d}}\right)$, with $A_{\mathrm{s}}=\pi\left(D_{\mathrm{d}}+\Delta D\right)^{2} / 4$ and $A_{\mathrm{d}}=\pi D_{\mathrm{d}}^{2} / 4$, respectively. Simulating $k_{1} \downarrow, k_{2} \downarrow$ caused a left-upward shift of the group-averaged $D-P$ curve, as well as a $40 \%$ in- 

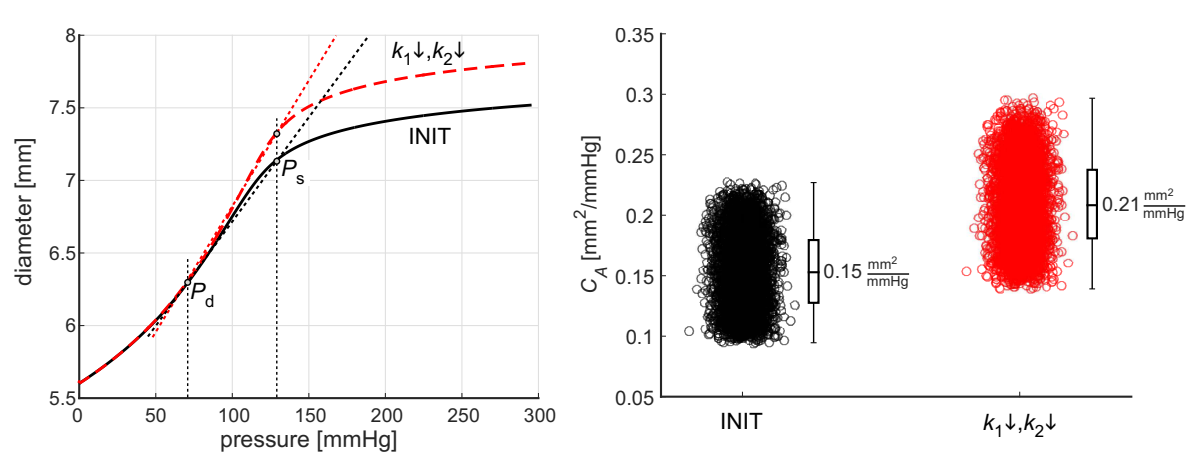

Figure 7.7: Left panel: Reducing collagen parameters $k_{1}$ and $k_{2}$ with $40 \%$ (i.e. $k_{1} \downarrow, k_{2} \downarrow$ ), caused a left-upward shift in the average diameter-pressure $(D-P)$ relation. Tangent lines (dotted lines) from the diastolic $D-P$ point to the systolic $D-P$ point indicate area compliance. Right panel: Simulating $k_{1} \downarrow, k_{2} \downarrow$ caused area compliance $\left(C_{A}\right)$ to increase from $0.15 \mathrm{~mm}^{2} \mathrm{mmHg}^{-1}$ to $0.21 \mathrm{~mm}^{2} \mathrm{mmHg}^{-1}$, respectively. For each INIT and $k_{1} \downarrow, k_{2} \downarrow$ realisation, area compliance was calculated using $C_{A}=\left(A_{\mathrm{s}}-A_{\mathrm{d}}\right) /\left(P_{\mathrm{s}}-P_{\mathrm{d}}\right)$, with $A_{\mathrm{s}}=\pi\left(D_{\mathrm{d}}+\Delta D\right)^{2} / 4$ and $A_{\mathrm{d}}=$ $\pi D_{\mathrm{d}}^{2} / 4$, respectively.

crease in $C_{A}$ (Fig. 7.7). In Fig. 7.6, distributions of $L_{\text {coll }}$ for the $k_{1} \downarrow, k_{2} \downarrow$ realisations (red dashed curves) are shown. We found $L_{\text {coll }}$ to equal $0.8[0.3 ; 2.5] \%$ at $P_{\mathrm{d}}, 1.7$ $[0.6 ; 5.0] \%$ at $P_{\mathrm{m}}$, and $10.8[7.2 ; 17.6] \%$ at $P_{\mathrm{s}}$ (median $\left[\mathrm{PCI}_{25 \rightarrow 75}\right]$ ). As compared to the INIT realisations, spread in $L_{\text {coll }}$ was lower for $k_{1} \downarrow, k_{2} \downarrow$ realisations (Fig. 7.6).

\subsection{Discussion}

Computational models of arterial wall mechanics could be valuable for predicting effects of vascular drug therapies on individual arterial wall constituents. The aim of this study was 1) to quantify how measurement noise propagates into uncertainty of the model predictions, and 2) to pinpoint the measurements responsible for the largest spread in mechanical characteristics. The relevance of the model output uncertainty was assessed by simulating the effects of vascular drug treatment on constituent load bearing. To our knowledge, this is the first study to perform rigorous uncertainty quantification and sensitivity analysis, assessing the influence of measurement noise in clinical arterial pressure and diameter measurements on constitutive model predictions.

The present study demonstrates that the clinically usefulness of estimating mechanical characteristics of the carotid artery using a constitutive model is hampered by measurement uncertainty. Using sensitivity analysis, we pinpointed that the majority of uncertainty in mechanical characteristics is caused by uncertainty in measurements of distension and IMT. 


\subsubsection{Model fitting and parameter estimation}

Model fitting was performed on single-exponential diameter-pressure curves calculated from diastolic and systolic diameters and pressures. The assumption of a markedly exponential diameter-pressure curve in the physiological (i.e. diastolic to systolic) pressure range was reported in earlier works by Hayashi et al. [11] and Meinders and Hoeks [24]. At a wider pressure range (i.e. 40 to $160 \mathrm{mmHg}$ ), a representative constitutive model realisation suggested sigmoidal diameterpressure behaviour (Fig. 7.4). Such sigmoidal behaviour was also observed in in vitro studies, performing inflation tests on human aortic segments and rat carotid arteries $[9,22]$.

In the present study, best-fit constitutive parameter values for elastin stiffness (i.e. 47.4 [44.8;50.0] $\mathrm{kPa}$ ) were in agreement to those found for cadaveric carotid arteries, reporting values between 20 and $60 \mathrm{kPa}[30,35]$. Moreover, values for collagen parameter $k_{2}$ were well within ranges found in earlier studies [30, 35]. We found a large spread for constitutive parameter $k_{1}$, governing collagen stiffness, i.e. median $\left[\mathrm{PCI}_{25 \rightarrow 75}\right]$ of $2.1[0.8 ; 5.9] \mathrm{kPa}$. Previous in vitro studies found $k_{1}$ values ranging from $2.9 \mathrm{kPa}$ to $99.9 \mathrm{kPa}$, respectively [35]. Therefore, our findings for $k_{1}$ are on the low end to those found in the aforementioned studies. It has been pointed out that appropriate choices of both $k_{1}$ and $k_{2}$ ensure collagen to virtually not bear load at very low amounts of stretch (i.e. at subphysiological pressure loads), whereas it will become the dominant load bearer at high amounts of stretch [15]. In the present study, and in in vivo studies per se, diameter and pressure measurements at these very low pressures are unavailable, making robust estimation of model parameters (particularly $k_{1}$ ) cumbersome, as illustrated by our findings.

\subsubsection{Sensitivity analysis}

Sensitivity analysis indicated that the most important contributors to uncertainty in $c_{\text {elast }}$ are both the variables measured by ultrasound (i.e. distension and IMT), whereas uncertainty in collagen parameter $k_{1}$ was primarily caused by measurement uncertainty of distension. Although our model-based approach still requires blood pressure to be measured, improving the precision of distension and wall thickness measurements clearly appears to be most rewarding.

Recent technological advances in vascular imaging, including plane wave ultrasound and image-reconstruction algorithms, could reduce the measurement noise of a single ultrasound measurement [2]. More practically, uncertainty in mechanical characteristics could be reduced by increasing the number of repeated measurements ( $N_{\text {rep }}$, Eq. 7.8). 


\subsubsection{Decreasing measurement uncertainty by increasing the number of repeated measurements}

We evaluated to which extent increasing $N_{\text {rep }}$, for all measured variables displayed in Table 7.1, influenced uncertainty in collagen load bearing parameters. Results indicate that increasing the number of repeated measurements from 3 to 10 decreases the spread in $L_{\text {coll }}$ by $\approx 50 \%$ (i.e. reducing the $25^{\text {th }}$ to $75^{\text {th }}$ percentile confidence interval at systolic blood pressure from $[12.7 ; 24.0] \%$ to $[14.0 ; 19.9] \%$, Table 7.5).

Table 7.5: The effect of increasing the number of repeated clinical measurements $\left(N_{\text {rep }}\right)$ on collagen load bearing parameters $\left(L_{\text {coll }}\right)$ as predicted using the initial constitutive model realisations

\begin{tabular}{lcccccc}
\cline { 2 - 7 } & \multicolumn{2}{c}{$L_{\text {coll }}$ at $P_{\mathrm{d}}$} & \multicolumn{2}{c}{$L_{\text {coll }}$ at $P_{\mathrm{m}}$} & \multicolumn{2}{c}{$L_{\text {coll }}$ at $P_{\mathrm{s}}$} \\
\hline$N_{\text {rep }}$ & median [\%] & $\mathrm{PCI}_{25 \rightarrow 75}[\%]$ & median [\%] & $\mathrm{PCI}_{25 \rightarrow 75}[\%]$ & median [\%] & $\mathrm{PCI}_{25 \rightarrow 75}[\%]$ \\
\hline 3 & 1.5 & {$[0.6 ; 4.5]$} & 3.1 & {$[1.2 ; 8.2]$} & 16.7 & {$[12.7 ; 24.0]$} \\
5 & 1.5 & {$[0.7 ; 3.5]$} & 3.0 & {$[1.4 ; 6.7]$} & 16.5 & {$[13.2 ; 21.8]$} \\
10 & 1.5 & {$[0.9 ; 2.7]$} & 3.0 & {$[1.8 ; 5.3]$} & 16.5 & {$[14.0 ; 19.9]$} \\
\hline
\end{tabular}

$\mathrm{PCI}_{25 \rightarrow 75}: 25^{\text {th }}$ to $75^{\text {th }}$ percentile confidence interval, $P_{\mathrm{d}}$ : diastolic blood pressure, $P_{\mathrm{m}}:$ mean blood pressure, and $P_{\mathrm{s}}$ : systolic blood pressure.

Based on the results of our sensitivity analysis, increasing the number repetitions of ultrasound measurements appears most rewarding in reducing uncertainty in collagen load bearing parameters.

\subsubsection{Model-based assessment of vascular drug therapies}

AGE-breaking vascular drug therapy was simulated by changing constitutive model parameters governing collagen behaviour (i.e. parameters $k_{1}$ and $k_{2}$ ). We chose to reduce collagen stress scaling parameter $k_{1}$, as well as collagen stresscurve shape parameter $k_{2}$ by $40 \%$ of their initial best-fit values. Consequently, the modelled collagen becomes incrementally less stiff at low amounts of strain, but will also stiffen 'later' (i.e. at higher amounts of strain), compared to when the initial parameters would be used (Fig. 7.6). This shift in stress-strain behaviour with decreasing cross-link densities was measured also in collagenous tissue such as the pericardium $[8,21]$. Reducing constitutive parameters $k_{1}$ and $k_{2}$ by $40 \%$, resulted in a model-predicted area compliance increase from $0.15 \mathrm{~mm}^{2} \mathrm{mmHg}^{-1}$ to $0.21 \mathrm{~mm}^{2} \mathrm{mmHg}^{-1}$ (Fig. 7.7). This observed $40 \%$ increase in area compliance is in agreement with in vitro measurements performed in rat carotid arteries, following AGE-breaker treatment [48]. Furthermore, collagen load bearing is reduced, with the load transferred to elastin instead (Fig. 7.7). The median reduction of collagen load bearing was $1 \%, 2 \%$, and $6 \%$ at diastolic, mean and systolic blood pressures (Fig. 7.5 and 7.6). However, the reduction in collagen load bearing was exceeded by the initial spread in collagen load bearing (Fig. 7.5). This is illustrated by the reduction in collagen load bearing at systolic blood pressure $(6 \%$, respectively, Fig. 
7.6), which is much smaller compared to the $25^{\text {th }}$ to $75^{\text {th }}$ percentile confidence interval ranging between $12.7 \%$ and $24.0 \%$, respectively (Fig. 7.6 ). In other words, measurement of the benefit of a cross-link breaker on arterial compliance may be easily concealed by uncertainties in the estimation of the effects, given the impact of noise on model output.

\subsubsection{Limitations}

Unfortunately, no actual distensibility measurements, acquired before and during AGE-breaker treatment were available in this study. Consequently, we had to resort to simulating AGE-breaker treatment using our constitutive model. This was achieved by changing constitutive parameters governing collagen behaviour, reproducing results from an earlier AGE-breaker intervention study [48].

The one-layered Holzapfel-Gasser-Ogden model we used - neglecting active smooth muscle response and containing only three parameters to characterise elastin and collagen behaviour - was chosen as a pragmatic simplification of the actual arterial biomechanical behaviour. Furthermore, our model neglects the dispersion of collagen fibre orientation in the adventitia [10]. We are aware that more elaborate models exist describing the influence of collagen cross-links on the stress-strain behaviour of an artery more directly. For example, in the work of Sáez et al. [30], a cross-linking degree parameter was introduced which includes crosslinks behaviour between the main collagen fibres. However, using such a model requires estimating one extra model parameter. To ensure unique parameter values, this would require more clinical data to be measured, which might not be possible in in vivo situations. Of note, parameter values in the current study were highly similar, indicated by the fact that the best-fit constitutive parameter values — for each of the 10 random starting points - were highly similar.

In our model, not all combinations of $c_{\text {elast }}, k_{1}$, and $k_{2}$ yield physiological behaviour. However, the adjustment (fitting) of these parameters ensures that eventually their combination does yield physiological behaviour. The parameter $c_{\text {elast }}$ can be physiologically interpreted as the stiffness of the arterial elastin. All fitted values for this parameter ranged from 35 to $55 \mathrm{kPa}$, which corresponds to previous literature [35]. For $k_{1}$ and $k_{2}$, no separate interpretation can be made in terms of physiology. Nevertheless, the fitted combinations of $k_{1}$ and $k_{2}$ yielded physiologically realistic mechanical behaviour which, given that the elastin model was plausibly parametrised, corresponds to realistic collagen behaviour.

The global variance-based sensitivity analysis method used, distinguishes from more commonly used local methods by taking into account the entire distribution of measured variables, being model free, and assessing interaction between measured variables $[5,29,40]$. However, it assumes on the one hand statistical independence between measured variables and on the other hand that variance is an adequate metric for model uncertainty. The latter assumption becomes questionable for skewed distributions of parameters, i.e. as present in the distributions of parameters $k_{1}$ and $L_{\text {coll }}$ at $P_{\mathrm{d}}$ and $P_{\mathrm{m}}$, respectively (Fig. 7.5 and 7.6). A solution to this problem could be to use moment-independent sensitivity methods instead 
[5]. To this end, Borgonovo [4] evaluated an alternative sensitivity metric that, instead of being computed using the variance of the model uncertainty distribution, considers this distribution as a whole. In their paper, it was concluded that sensitivity indices of both methods 1) show discrepancies between influential measured variables, but 2) agree in distinguishing non-influential from influential measured variables [4]. Based on these findings, we believe that utilising the variance-based sensitivity analysis as proposed by Quicken et al. [29] in this study is justified.

\subsubsection{Conclusion}

This study shows that in vivo assessment of arterial wall mechanics using a constitutive model is hampered by large model uncertainty. We quantified model uncertainty in constitutive parameters (i.e. $c_{\text {elast }}, k_{1}$, and $k_{2}$, respectively), and collagen load bearing parameters, at various blood pressures ( $\left.L_{\text {coll }}\right)$. Our simulation of vascular drug therapy suggested a reduction of collagen load bearing of 6-percentage points, at systolic blood pressure. This reduction is 3 to 4 times lower compared to its uncertainty. Therefore, model output uncertainty could conceal potential effects of vascular drugs. Sensitivity analysis revealed that estimation of mechanical characteristics would benefit most from increasing the precision of measurements of arterial diameter, distension, and wall thickness. Whereas the potential for improving the precision of e.g. a single ultrasound measure is practically limited, the effective precision of ultrasound measurements could be improved by increasing the number of repeated measurements.

\section{Acknowledgments}

This study was funded by a Kootstra Talent Fellowship awarded to M.H.G. Heusinkveld by Maastricht University Medical Centre, S. Quicken is paid by Chemelot InSciTe, R.J. Holtackers received funding from Stichting de Weijerhorst, and B. Spronck was funded by an Endeavour Research Fellowship from the Australian government. 


\section{A1 Appendix: Constitutive model}

\section{A1.1 Constitutive relations}

Under the assumption that 1) elastin and collagen act in parallel in terms of mechanical load-bearing; 2) the vessel wall is incompresible, and 3) deformation occurs only along the principal axes, the constituent-specific strain energy functions (Eq. 7.1 and 7.2) were cast into a relation describing local Cauchy stress:

$$
\sigma_{n}=-P_{\mathrm{h}}+\lambda_{n} \frac{\partial W_{\text {elast }}}{\partial \lambda_{n}}+\lambda_{n} \frac{\partial W_{\text {coll }}}{\partial \lambda_{n}},
$$

The subscript $n$ is $r r$ for the radial direction, $\theta \theta$ for the circumferential direction and $z z$ for the axial direction. This results in the following expressions for $\sigma_{r r}$ $\sigma_{\theta \theta}$ and $\sigma_{z z}$ :

$$
\begin{aligned}
& \sigma_{r r}=-P_{\mathrm{h}}+2 \lambda_{r r}^{2} c_{\text {elast }}, \\
& \sigma_{\theta \theta}=-P_{\mathrm{h}}+2 \lambda_{\theta \theta}^{2} c_{\text {elast }}+4 k_{1} \lambda_{\theta \theta}^{2}\left(\lambda_{\text {fibre }}^{2}-1\right) \cos ^{2}\left(\beta_{0}\right)\left(\exp \left[k_{2}\left(\lambda_{\text {fibre }}^{2}-1\right)^{2}\right]\right) \text { (7.A2) } \\
& \sigma_{z z}=-P_{\mathrm{h}}+2 \lambda_{z z}^{2} c_{\text {elast }}+4 k_{1} \lambda_{z z}^{2}\left(\lambda_{\text {fibre }}^{2}-1\right) \sin ^{2}\left(\beta_{0}\right)\left(\exp \left[k_{2}\left(\lambda_{\text {fibre }}^{2}-1\right)^{2}\right]\right),
\end{aligned}
$$

with $P_{\mathrm{h}}$ the local hydrostatic pressure within the wall, $\lambda_{\text {fibre }}$ the fibre stretch given by $\lambda_{\text {fibre }}=\sqrt{\cos ^{2}\left(\beta_{0}\right) \lambda_{\theta \theta}^{2}+\sin ^{2}\left(\beta_{0}\right) \lambda_{z z}^{2}}, \beta_{0}$ the fibre helix angle, $c_{\text {elast }}$ the constitutive (stiffness) parameter of elastin, and $k_{1}$ and $k_{2}$ stiffness parameters of collagen, respectively.

\section{A1.2 Constitutive framework}

We distinguished between the unstressed and stressed states of an artery (Fig. 7.2). Under the assumption of an incompressible wall tissue, a mapping of coordinates $(r, \theta, z)$ of the deformed, stressed configuration to coordinates $(R, \Theta, Z)$ of the unstressed configuration is given by:

$$
r(R)=\sqrt{r_{\mathrm{o}}^{2}-\frac{R_{\mathrm{o}}^{2}-R^{2}}{\lambda_{z z} k_{\alpha}}}, \theta=k_{\alpha} \Theta, \text { and } z=\frac{l}{L} Z,
$$

where $R_{\mathrm{o}}$ and $r_{\mathrm{o}}$ are the outer radii of respectively the unstressed and stressed configuration. The parameter $k_{\alpha}$ is defined as $k_{\alpha}=\frac{2 \pi}{2 \pi-\alpha}$, with $\alpha$ the opening angle (Fig. 7.2). $L$ and $l$ are vessel length of the unstressed and stressed configuration defining the initial pre-stretch of an artery $\lambda_{z z}$. The principal stretch ratios 
$\left(\lambda_{r r}, \lambda_{\theta \theta}, \lambda_{z z}\right)$ with respect to the unstressed configuration are:

$$
\lambda_{r r}=\frac{R}{r k_{\alpha} \lambda_{z z}}, \quad \lambda_{\theta \theta}=\frac{k_{\alpha} r}{R}, \quad \text { and } \quad \lambda_{z z}=\frac{l}{L} .
$$

Inner and outer radii ( $r_{\mathrm{i}}$ and $r_{\mathrm{o}}$, respectively) in the stressed configuration can be written as follows:

$$
r_{\mathrm{i}}=\frac{\lambda_{\theta \theta} R_{\mathrm{i}}}{k_{\alpha}} \quad \text { and } \quad r_{\mathrm{o}}=\sqrt{\frac{A_{\mathrm{w}}}{\pi}+r_{\mathrm{i}}^{2}},
$$

where $A_{\mathrm{w}}$ is the cross-sectional wall area in the stressed configuration (Fig. 7.2). In practice, $A_{\mathrm{w}}$ can be determined by measuring IMT and vessel diameter using ultrasonography [12]. We furthermore assumed $\lambda_{z z}=1.20, \alpha=100^{\circ}$, and $\beta_{0}=$ $35.3^{\circ}$.

\section{A1.3 Balance equations}

Conservation of momentum is given by

$$
\nabla \cdot \boldsymbol{\sigma}=\mathbf{0}
$$

with $\nabla$ the spatial gradient operator. Assuming axisymmetry and neglecting torsion, acceleration forces, body forces and axial extension, Eq. 7.A8 reduces to

$$
\frac{\partial \sigma_{r r}}{\partial r}+\frac{\sigma_{r r}-\sigma_{\theta \theta}}{r}=0 .
$$

Applying the boundary conditions $\sigma_{r r}\left(r_{\mathrm{i}}\right)=-P$ and $\sigma_{r r}\left(r_{\mathrm{o}}\right)=0$, the expression for lumen pressure $(P)$ is given by

$$
P=\int_{r_{i}}^{r_{o}} \frac{\sigma_{\theta \theta}-\sigma_{r r}}{r} \mathrm{~d} r .
$$

Reduced axial force is given by

$$
F_{z}=\pi \int_{r_{i}}^{r_{o}}\left(2 \sigma_{z z}-\sigma_{r r}-\sigma_{\theta \theta}\right) r \mathrm{~d} r .
$$

\section{A1.4 Calculating collagen load bearing parameters}

We calculated collagen load bearing $\left(L_{\text {coll }}\right)$ at three blood pressure levels; diastolic, mean and systolic blood pressure (i.e. $P_{\mathrm{d}}, P_{\mathrm{m}}$, and $P_{\mathrm{s}}$, respectively). Mean blood pressure $\left(P_{\mathrm{m}}\right)$ was calculated as $\frac{1}{3} P_{\mathrm{s}}+\frac{2}{3} P_{\mathrm{d}}$. Using Eq. 7.A2, the expression for $L_{\text {coll }}$ is given by 


$$
L_{\text {coll }}=\left.\int_{r_{\mathrm{i}}}^{r_{\mathrm{o}}} \frac{\tau_{\theta \theta, \text { coll }}}{\sigma_{\theta \theta}-\sigma_{r r}} \mathrm{~d} r\right|_{P \in\left\{P_{\mathrm{d}}, P_{\mathrm{m}}, P_{\mathrm{s}}\right\}} \cdot 100 \%,
$$

with $\tau_{\theta \theta \text {,coll }}$ the circumferential stress borne by collagen, given by

$$
\tau_{\theta \theta, \mathrm{coll}}=\lambda_{\theta \theta} \frac{\partial W_{\mathrm{coll}}}{\partial \lambda_{\theta \theta}} .
$$




\section{References}

[1] S. Avril, P. Badel, M. Gabr, M. A. Sutton, and S. M. Lessner. Biomechanics of porcine renal arteries and role of axial stretch. Journal of Biomechanical Engineering, 135(8):081007, 2013.

[2] A. Besson, M. Zhang, F. Varray, H. Liebgott, D. Friboulet, Y. Wiaux, J.-P. Thiran, R. E. Carrillo, and O. Bernard. A sparse reconstruction framework for Fourier-based plane-wave imaging. IEEE Transactions on Ultrasonics, Ferroelectrics, and Frequency Control, 63(12):2092-2106, 2016.

[3] J. M. Bland and D. G. Altman. Statistics notes: measurement error. BMJ, 313(7059):744, 1996.

[4] E. Borgonovo. A new uncertainty importance measure. Reliability Engineering \& System Safety, 92(6):771-784, 2007.

[5] E. Borgonovo and E. Plischke. Sensitivity analysis: a review of recent advances. European Journal of Operational Research, 248(3):869-887, 2016.

[6] M. Brownlee, M D. Advanced protein glycosylation in diabetes and aging. Annual Review of Medicine, 46(1):223-234, 1995.

[7] L. Engelen, C. D. A. Stehouwer, and C. G. Schalkwijk. Current therapeutic interventions in the glycation pathway: evidence from clinical studies. Diabetes, Obesity and Metabolism, 15(8): 677-689, 2013.

[8] P. Fratzl. Collagen: structure and mechanics. Springer Science \& Business Media, 2008.

[9] P. Fridez, M. Zulliger, F. Bobard, G. Montorzi, H. Miyazaki, K. Hayashi, and N. Stergiopulos. Geometrical, functional, and histomorphometric adaptation of rat carotid artery in induced hypertension. J Biomech, 36(5):671-680, May 2003.

[10] T. C. Gasser, R. W. Ogden, and G. A. Holzapfel. Hyperelastic modelling of arterial layers with distributed collagen fibre orientations. Journal of the royal society interface, 3(6):15-35, 2006.

[11] K. Hayashi, H. Handa, S. Nagasawa, A. Okumura, and K. Moritake. Stiffness and elastic behavior of human intracranial and extracranial arteries. Journal of Biomechanics, 13(2):175-184, 1980.

[12] A. P. G. Hoeks, P. J. Brands, J. M. Willigers, and R. S. Reneman. Non-invasive measurement of mechanical properties of arteries in health and disease. Proceedings Of The Institution Of Mechanical Engineers Part H, 213(3):195-202, 1999.

[13] R. J. Holtackers, B. Spronck, M. H. G. Heusinkveld, G. Crombag, J. Op 't Roodt, T. Delhaas, M. E. Kooi, K. D. Reesink, and E. Hermeling. Head orientation should be considered in ultrasound studies on carotid artery distensibility. Journal of Hypertension, 34(8):1551-5, Aug 2016.

[14] G. A. Holzapfel and R. W. Ogden. Constitutive modelling of arteries. Proceedings of the Royal Society A, 466(2118):1551-1597, 2010.

[15] G. A. Holzapfel, T. C. Gasser, and R. W. Ogden. A new constitutive framework for arterial wall mechanics and a comparative study of material models. Journal of Elasticity, 61(1-3):1-48, 2000.

[16] G. A. Holzapfel, T. C. Gasser, and M. Stadler. A structural model for the viscoelastic behavior of arterial walls: Continuum formulation and finite element analysis. European Journal of Mechanics - A/Solids, 21(3):441 - 463, 2002. 
[17] W. Huberts, W. P. Donders, T. Delhaas, and F. N. van de Vosse. Applicability of the polynomial chaos expansion method for personalization of a cardiovascular pulse wave propagation model. International Journal for Numerical Methods in Biomedical Engineering, 30(12):1679-1704, 2014.

[18] J. D. Humphrey. Cardiovascular solid mechanics: cells, tissues, and organs. Springer Science \& Business Media, New York, United States of America, 2002.

[19] J. D. Humphrey, D. G. Harrison, C. A. Figueroa, P. Lacolley, and S. Laurent. Central artery stiffness in hypertension and aging a problem with cause and consequence. Circulation Research, 118(3): 379-381, 2016.

[20] D. A. Kass, E. P. Shapiro, M. Kawaguchi, A. R. Capriotti, A. Scuteri, and E. G. Lakatta. Improved arterial compliance by a novel advanced glycation end-product crosslink breaker. Circulation, 104(13):1464-1470, 2001.

[21] H. R. Kayed, N. Kirby, A. Hawley, S. T. Mudie, and R. G. Haverkamp. Collagen fibril strain, recruitment and orientation for pericardium under tension and the effect of cross links. RSC Advances, 5(125):103703-103712, 2015.

[22] G. J. Langewouters, K. H. Wesseling, and W. J. A. Goedhard. The static elastic properties of 45 human thoracic and 20 abdominal aortas in vitro and the parameters of a new model. Journal of Biomechanics, 17(6):425-435, 1984.

[23] M. McNulty, A. Mahmud, and J. Feely. Advanced glycation end-products and arterial stiffness in hypertension. American Journal of Hypertension, 20(3):242-247, 2007.

[24] J. M. Meinders and A. P. G. Hoeks. Simultaneous assessment of diameter and pressure waveforms in the carotid artery. Ultrasound in Medicine \& Biology, 30(2):147-154, 2004.

[25] J. J. Moré and D. C. Sorensen. Computing a trust region step. SIAM Journal on Scientific and Statistical Computing, 4(3):553-572, 1983.

[26] National Research Council. Assessing the reliability of complex models: mathematical and statistical foundations of verification, validation, and uncertainty quantification. National Academies Press, 2012.

[27] M. F. O'Rourke and J. Hashimoto. Mechanical factors in arterial aging: a clinical perspective. Journal of the American College of Cardiology, 50(1):1-13, 2007.

[28] D. J. Patel, D. L. Fry, and J. S. Janicki. The elastic symmetry of arterial segments in dogs. Circulation Research, 24(1):1-8, 1969.

[29] S. Quicken, W. P. Donders, E. M. J. van Disseldorp, K. Gashi, B. M. E. Mees, F. N. van de Vosse, R. G. P. Lopata, T. Delhaas, and W. Huberts. Application of an adaptive polynomial chaos expansion on computationally expensive three-dimensional cardiovascular models for uncertainty quantification and sensitivity analysis. Journal of Biomechanical Engineering, 138(12):121010, 2016.

[30] P. Sáez, E. Peña, and M. A. Martínez. A structural approach including the behavior of collagen cross-links to model patient-specific human carotid arteries. Annals of Biomedical Engineering, 42(6):1158-1169, 2014.

[31] A. Saltelli, M. Ratto, T. Andres, F. Campolongo, J. Cariboni, D. Gatelli, M. Saisana, and S. Tarantola. Global sensitivity analysis: the primer. John Wiley \& Sons, Chichester, United Kingdom, 2008.

[32] R. E. Shadwick. Mechanical design in arteries. Journal of Experimental Biology, 202(23): 3305-3313, 1999. 
[33] B. W. Silverman. Density Estimation for Statistics and Data Analysis. Chapman \& Hall, London, United Kingdom, 1st edition, 1986.

[34] I. M. Sobol. On the distribution of points in a cube and the approximate evaluation of integrals. Zhurnal Vychislitel'noi Matematiki i Matematicheskoi Fiziki, 7(4):784-802, 1967.

[35] G. Sommer and G. A. Holzapfel. 3D constitutive modeling of the biaxial mechanical response of intact and layer-dissected human carotid arteries. Journal of the Mechanical Behavior of Biomedical Materials, 5(1):116-128, 2012.

[36] G. Sommer, P. Regitnig, L. Költringer, and G. A. Holzapfel. Biaxial mechanical properties of intact and layer-dissected human carotid arteries at physiological and supraphysiological loadings. American Journal of Physiology-Cell Physiology, 298(3):H898, 2010.

[37] B. Spronck, M. H. G. Heusinkveld, W. P. Donders, A. G. W. de Lepper, J. Op’t Roodt, A. A. Kroon, T. Delhaas, and K. D. Reesink. A constitutive modeling interpretation of the relationship between carotid artery stiffness, blood pressure and age in hypertensive subjects. American Journal of Physiology-Heart and Circulatory Physiology, 308:H568-H582, 2015.

[38] B. Spronck, M. H. G. Heusinkveld, F. H. Vanmolkot, J. Op 't Roodt, E. Hermeling, T. Delhaas, A. A. Kroon, and K. D. Reesink. Pressure-dependence of arterial stiffness: potential clinical implications. Journal of Hypertension, 33(2):330-338, 2015.

[39] B. Sudret. Global sensitivity analysis using polynomial chaos expansions. Reliability Engineering \& System Safety, 93(7):964-979, 2008.

[40] B. Sudret. Polynomials chaos expansions and stochastic finite element methods. Risk and Reliability in Geotechnical Engineering, 2015.

[41] K. Takamizawa and K. Hayashi. Strain energy density function and uniform strain hypothesis for arterial mechanics. Journal of Biomechanics, 20(1):7-17, 1987.

[42] R. R. Townsend, I. B. Wilkinson, E. L. Schiffrin, A. P. Avolio, J. A. Chirinos, J. R. Cockcroft, K. S. Heffernan, E. G. Lakatta, C. M. McEniery, G. F. Mitchell, S. S. Najjar, W. W. Nichols, E. M. Urbina, and T. Weber. Recommendations for improving and standardizing vascular research on arterial stiffness: A scientific statement from the American Heart Association. Hypertension, Jul 2015.

[43] A. van der Horst, C. N. van den Broek, F. N. van de Vosse, and M. C. M. Rutten. The fiber orientation in the coronary arterial wall at physiological loading evaluated with a two-fiber constitutive model. Biomechanics and Modeling in Mechanobiology, 11(3):533-542, 2012.

[44] P. Van Loon. Length-force and volume-pressure relationships of arteries. Biorheology, 14(4): 181-201, 1976.

[45] P. N. Watton, Y. Ventikos, and G. A. Holzapfel. Modelling the mechanical response of elastin for arterial tissue. Journal of Biomechanics, 42(9):1320-1325, 2009.

[46] H. W. Weizsäcker, H. Lambert, and K. Pascale. Analysis of the passive mechanical properties of rat carotid arteries. Journal of Biomechanics, 16(9):703-715, 1983.

[47] C. Willekes, A. P. G. Hoeks, M. L. Bots, P. J. Brands, J. M. Willigers, and R. S. Reneman. Evaluation of off-line automated intima-media thickness detection of the common carotid artery based on m-line signal processing. Ultrasound in Medicine \& Biology, 25(1):57-64, 1999.

[48] B. H. Wolffenbuttel, C. M. Boulanger, F. R. Crijns, M. S. Huijberts, P. Poitevin, G. N. Swennen, S. Vasan, J. J. Egan, P. Ulrich, A. Cerami, and B. I. Lévy. Breakers of advanced glycation end products restore large artery properties in experimental diabetes. Proceedings of the National academy of Sciences of the United States of America, 95(8):4630-4634, Apr 1998. 

General discussion 


\subsection{Executive summary}

$\mathrm{N}$ this thesis, we focussed on developing a computational modelling platform

1 to assess heart-vessel interaction in humans.

We first performed a meta-analysis of clinical studies to explore a conceptual chain-of-events describing how increases in arterial stiffness lead to increases in systolic blood pressure with consequent left ventricular mass increases. Based on the findings as well as the limitations drawn from our meta-analysis, we then turned our attention to developing computational models and applying them to specific research questions. We developed a computational modelling platform describing the heart and vasculature, consisting on the one hand of a well characterised and validated whole-heart model, and on the other hand a newly developed vascular module describing wave transmission in blood vessels. This platform was subsequently used to assess the validity of augmentation index, a metric that is hypothesised to capture the deleterious effect of increased wave reflection, and earlier arrival of wave reflections on left ventricular (LV) work.

Parallel to using computational models for hypothesis-testing, we were interested in using our platforms for patient-specific applications. A caveat of patient-specific modelling is missing data. To benefit patient-specific modelling, we evaluated the role of incorporating physical-physiological adaptation rules into models to reduce the amount of clinical data needed to render these models patient-specific.

Following model development and assessment steps, we employed models in two use-cases concerning patient-specific modelling: i) characterising LV (diastolic) function, which is highly relevant in heart failure with preserved ejection fraction (HFpEF) and ii) constitutive interpretation of arterial stiffness measurement, which is key to treatment monitoring.

\subsection{Left ventricular mass, arterial stiffness and systolic blood pressure: Exploring the 'Bermuda Triangle'}

As a first step, we performed a meta-analysis to assess clinical evidence describing changes in indices of cardiac- and vascular structure and function following intervention, i.e. conventional antihypertensive treatment, bariatric surgery or lifestyle interventions. Pooled analysis of clinical data suggested a significant association between treatment-induced changes in arterial stiffness (assessed by pulse wave velocity (PWV)) on changes in LV mass (assessed by LV mass index (LVMI)). This association was characterised by a $6.9 \mathrm{~g} \mathrm{~m}^{-2}$ decrease in LVMI per 1 $\mathrm{m} \mathrm{s}^{-1}$ decrease in PWV (Chapter 2).

Haider et al. [23] established that in subjects with LV hypertrophy (LVH) an $\mathrm{LV}$ mass increase of $50 \mathrm{~g} \mathrm{~m}^{-1}$ (i.e. LV mass indexed by body height) increases the relative risk of cardiovascular disease by approximately two-fold. Studies included 
in our review reported PWV changes of approximately 1 to $3 \mathrm{~m} \mathrm{~s}^{-1}$, whereas LVMI changes were approximately to 2 to $35 \mathrm{~g} \mathrm{~m}^{-2}$ (Chapter 2). Our findings thus corroborate that arterial stiffness may be a major player in the above described chain-of-events leading to LV hypertrophy.

In the proposed chain-of-events, it is expected that systolic blood pressure is important as well, because increased arterial stiffness has been described as a cause and consequence of increased systolic blood pressure [12, 30, 56]. Furthermore, increased LV mass may result from independent mechanisms including increased arterial stiffness (e.g. through earlier arrival of pulse wave reflections) as well as increased systolic blood pressure (e.g. through decreased aortic compliance) $[8,24,42]$.

Unfortunately, we were unable to uncover sequential relations in this chain using our data extracted from clinical intervention studies. The editorial commentary on our review article used the term 'Bermuda Triangle' [59] as analogy to express the obscure mutual relations between arterial stiffness, systolic blood pressure, and LV mass. Interestingly, the authors [59] do justice to their analogy by glossing over the essential difference between diastolic blood pressure and systolic blood pressure. Mechanistically, systolic (i.e. 'peak') blood pressure determines peak wall stress and, therefore, is a known trigger for LV hypertrophy $[19,22,51]$.

Based on our findings from Chapter 2, four key issues related to the interpretation of corresponding clinical data and studies may be identified:

- First, the number of follow-up visits throughout intervention was insufficient to infer sequential relations between LV mass and the various haemodynamic indices reported. Meta-analyses of clinical studies appear unsuited to establish causality [1].

- Second, not all data we hoped to find was reported: studies that related left ventricular diastolic function to (changes) in arterial stiffness were scarce.

- Third, data on arterial stiffness changes were limited to reporting functional indices, (e.g. distensibility or pulse wave velocity), and did not consider constitutive/structural properties.

- Fourth, the clinical characterisation of e.g. LV mass and PWV contains measurement noise, which influences the associations found.

\subsection{Applications}

In this section, we place the findings from our model application studies in a broader perspective. We structure this discussion on the applications as follows: 1) cardiac, 2) vascular, and 3) heart-vessel interaction applications. Future research directions are discussed for each category separately. 


\subsubsection{Model-based estimation of left ventricular diastolic function indices: Towards non-invasive cardiac catheterisation}

We developed a non-invasive computational approach to obtain markers of LV diastolic abnormalities consisting of the metrics LV end-diastolic pressure $\left(p_{\text {ed }}\right)$ and compliance $\left(C_{\text {ed }}\right.$ ) (Chapter 6 ). We tested our model-estimations against data obtained by means of LV pressure-conductance catheterisation, and found reasonable agreement between model and measurement.

The patient population studied in Chapter $6(n=39)$ was homogeneous, containing too few patients with HFpEF according to current guidelines [43]. Therefore, it was not possible to examine whether and how $p_{\text {ed }}$ and $C_{\text {ed }}$ differ between HFpEF and control subjects, nor could we compare our model estimates against common echocardiographic indices, such as E/e', that are currently being used to distinguish between normal and elevated $p_{\text {ed }}$ [43]. Exploitation of an existing case-control study including echocardiography and blood pressure measurements, such as the Queen of Hearts study [11], would help in judging the 'face value' of model-based $p_{\text {ed }}$ and $C_{\text {ed }}$.

As a next step, a randomised controlled trial (RCT) is needed to prove the added value of $p_{\text {ed }}$ and $C_{\text {ed }}$ in classifying HFpEF or diastolic dysfunction. Previously, Zonnebeld et al. [64] proposed an RCT design to assess the added value of computational model predictions to optimise vascular access surgery in haemodialysis patients. Their study design consisted of routine, and routine complemented with model-prediction arms. Such a design would be realistic to test our approach.

Further development may include considering measurements of both baseline and Valsalva in our model-fitting scheme. Such approach, effectively doubling the amount of data available for model-fitting could improve model-estimations. Regarding modelling aspects herein, integration of this data requires mimicking Valsalva circumstances in CircAdapt. Unpublished numerical work, in which we were engaged previously, indicated feasibility of simulating Valsalva using our CircAdapt model. Simulated LV pressure and volume signals during Valsalva showed typical responses as compared to measured ones. Integration of haemodynamic measurements acquired during Valsalva in our model-based approach, demands strict adherence to a standardised Valsalva protocol (e.g. in terms of intra-oral pressure and straining duration, Chapter 6).

\subsubsection{Expanded view of vascular haemodynamics and wall mechanics: Towards in silico assessment of the performance of novel drug therapies}

Vascular haemodynamics and wall mechanics were studied using models with varying levels of (anatomical) detail. Our vascular module (Chapter 3) describes pressure-flow wave transmission in extensive arterial and venous networks. Although our model's numerical implementation differs from those used in the 
existing wave propagation modelling literature $[6,34,41,50]$, we found good agreement between our model's pressure and flow waveforms and those of a previously validated pulse wave propagation model $[6,34]$, i.e. relative errors in pressure and flow waveforms $\leq 5.6 \%$.

The constitutive model we used (Chapter 7), assuming a single-layered mixture of elastin and collagen, provides three-dimensional stresses and strains of these microstructural wall components. Constitutive parameter estimation using our model was feasible, and estimated constitutive parameters were in agreement with those reported in literature [53].

We introduced a vascular adaptation model (Chapter 5) to facilitate personalisation of vascular networks for use in patient-specific modelling applications. Our vascular adaptation model adequately predicted arterial radius and wall thickness as concluded from our test-case in which we compared model-estimated arterial geometries with those measured using ultrasound and MRI in healthy young volunteers (bias $\pm 2 \mathrm{SD}$ of difference equal to $0.2 \pm 2.6 \mathrm{~mm}$, and $-140 \pm 557$ $\mu \mathrm{m}$, respectively).

Observed changes in aortic (systolic) blood pressure with ageing are attributed to changes in aortic compliance or wave reflections [5, 40,44]. Models describing vascular wave transmission could be used to apportion LV mass changes to changes in compliance or wave reflections. Previous studies simulated arterial haemodynamics in virtual patient cohorts, e.g. to study changes in the aortic pressure pulse during ageing, or to assess the validity of metrics deemed a proxy for arterial stiffness $[46,60,61]$. To create large numbers of virtual haemodynamic models that represent virtual patients, perturbations of vascular segment geometries were imposed based on population-average values [60, 61]. To facilitate research using virtual patient cohorts, the basic concept of our vascular adaptation model may aid physiological parametrisation of subject-specific vascular trees.

In our analysis testing the applicability of incorporating vascular adaptation in haemodynamic models (Chapter 3), discrepancies between model-estimated geometries and measured data may result from limitations in the model of vascular adaptation. Our model is formulated based on the hypothesis of a homeostatic feedback mechanism that triggers changes in vascular geometry [31, 37, 39, 63]. Based on experimental data, we proposed a vascular adaptation model in which we assumed complete normalisation of wall (shear) stresses to homeostatic values. It has been suggested that the assumption of wall shear stress normalisation may become invalid for specific patients or patient groups in which the adaptive capacity of blood vessels is compromised (e.g. in diabetes or end-stage renal disease $[7,10,15])$. As consequence, applying the concept of adaptation may be inapplicable in some cases. One way to deal with issues of applicability is to estimate the adaptive capacity of blood vessels on beforehand, for instance using existing non-invasive tests such as flow-mediated dilation (FMD), because FMDscores have been shown in arterio-venous fistula creation studies to correlate with blood vessels' ability to remodel $[3,45]$.

In our vascular module, we used a mathematical relation (i.e. referred to as 
'tube law') for solving for pressures and flows, that relates blood pressure and vessel area. This tube law mimics the elastic behaviour of the vascular wall as observed in experiments by modelling an incremental increase in vessel stiffness for an incremental increase in pressure (Fig. 8.1). For the physiological pressure range (approximately 50 to $180 \mathrm{mmHg}$, Fig. 8.1), choosing appropriate stiffness values (i.e. $k$-values, Fig. 8.1) for the tube law resulted in excellent agreement with pressure-radius experiments of human arteries. For pressure loads below 50 mmHg or above $180 \mathrm{mmHg}$, deviations between tube law (coloured curves) and experiment (black curves) are observed, which limits our tube law's application to (sub-)physiological pressure ranges.

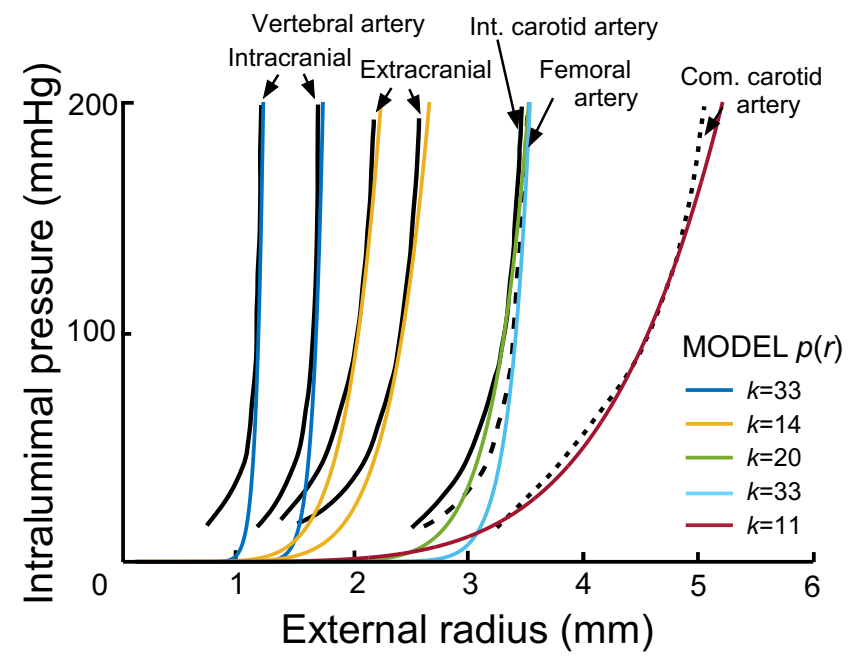

Figure 8.1: Estimated pressure-radius curves using tube law (coloured curves) drawn over previously measured data of human arteries (black solid, dashed and dotted curves, respectively). Measured data were taken from ex vivo inflation experiments on human arteries [25].

Ex vivo inflation experiments of human and animal arteries over a wide pressure range (e.g. 0 to $200 \mathrm{mmHg}$ ) clearly indicated S-shaped ('sigmoidal') pressure-area relations $[18,36]$. Such behaviour cannot be captured using our tube law (Fig. 8.1), but requires more elaborate mathematical functions [36], or a constitutive model description of a blood vessel's behaviour [27].

For some applications, consideration of microstructural properties of the arterial wall in wave transmission models may be necessary, because the vessel wall microstructure influences pressure-flow waves propagation [2].

For example, much research has been conducted in the field of drug development to reverse ageing-related stiffening of arteries $[16,33,58]$. Drugs that were developed include arterial wall collagen cross-link breakers (e.g. alagebrium chloride) that aim to reduce arterial stiffness by cleaving cross-links, thereby reducing 
vessel wall material stiffness. These drugs showed beneficial effects on compliance in rats [62]. Since cross-link breakers reportedly affect large artery stiffness, that of small arteries, but also cardiac mass $[17,55,62]$ the question, yet again, arises which local compliance or distributed effects of wave propagation play in their reported beneficial effect.

Based on the work performed in the thesis (Chapters 3, 5 and 7), creating an interface of on the one hand personalised constituent-based models of arterial wall mechanics and on the other hand our vascular module may serve as starting point for studies assessing effectivity of such novel vascular drugs.

Because significant uncertainty in model-estimates of collagen load-bearing was found, primarily due to measurement error in ultrasound data (Chapter 7), we speculated on methods to improve estimation of model predictions [54]. Holtackers et al. [28], demonstrated that local carotid artery distensibility significantly changed when young healthy volunteers were measured under two different (standardised) head positions. This finding may be exploited to effectively double the amount of mechanical information that can be employed for model fitting, by performing measurements at two head positions. Incorporating measurements, acquired at two axial stretch levels ('in vivo biaxial testing'), could thus in future serve as a utility to reduce the uncertainty in constitutive property estimation [49].

\subsubsection{Simulating heart-vessel interaction: Blood pressure characteristics, left ventricular hypertrophy, and left ventricular diastolic function}

We extended the existing CircAdapt whole-heart model [57] with a vascular module and investigated heart-vessel interaction by calculating on the one hand wave intensity tracings, and on the other hand augmentation index (AIx) (Chapters 3 and 4). We found that wave intensity tracings, as derived from our model simulations of local pressure and flow velocity corresponded to clinical measurements [29]. As such, we concluded that our model is suited for studying mechanical linkage between cardiac mechanics and vascular haemodynamics at the level of wave reflections. The advantage of a model of heart-vessel interaction is that it can provide noise-free and controllable study conditions. These characteristics are desirable when performing mechanistic studies, because measurement noise limits determination of wave transit delays, which are about 1 to 20 milliseconds $[26,48]$.

Patient-studies showed that concentric left ventricular remodelling and $\mathrm{LVH}$ are common findings in hypertension $[13,20]$. Therefore, consideration of LV afterload in relation to LV structure and function (i.e. heart-vessel interaction) appears to be important for research on treating LVH. Whereas findings from Chapter 2 offer insight in correlates of left ventricular hypertrophy, it does not elucidate the mechanism leading to left ventricular hypertrophy.

We justified using our circulatory model for heart-vessel interaction hypothesis 
testing, based on successful model validation and illustration of its applicability in clinically relevant use-cases (Chapters 3 and 4). In a preliminary study, we expanded the circulatory model with a model describing cardiac adaptation [4]. We then simulated the effect of stiffer arteries in our model. Computer simulations of haemodynamics were obtained under a reference normotensive (REF) state and a chronic hypertensive (HYP). Comparing left ventricular mass and pulse wave velocity before and after adaptation to a systemic hypertensive situation, we found a REF-HYP change $(\Delta)$ in LVMI of $-23 \mathrm{~g} \mathrm{~m}^{-2}$, as well as a difference in PWV of $-2.2 \mathrm{~m} \mathrm{~s}^{-1}$. Interestingly, the model-predicted increase of LV mass in hypertension finding fits well with the association between $\triangle \mathrm{LVMI}$ and $\triangle \mathrm{PWV}$ as extracted from clinical intervention studies (Fig. 8.2).

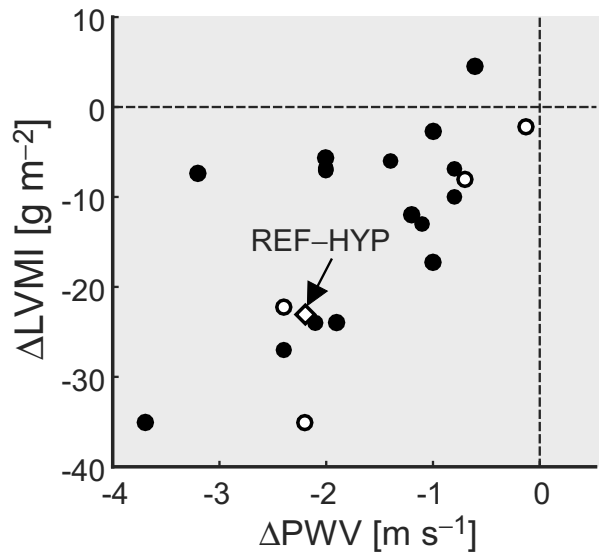

Figure 8.2: Change in pulse wave velocity $(\Delta \mathrm{PWV})$ and left ventricular mass index $(\Delta \mathrm{LVMI})$ values in clinical studies (circles) and simulation (diamond). Closed circles: antihypertensive drug therapy studies. Open circles: other types of intervention studies, e.g. bariatric surgery. Diamond: Simulated relation between $\triangle \mathrm{PWV}$ and $\triangle$ LVMI obtained by subtraction of systemic hypertension simulation (HYP) values from the reference simulation (REF) values.

Future modelling work should aim for mechanistic hypothesis-testing of the effect of earlier arrival of pulse wave reflections (e.g. using wave intensity analysis) or evaluation of interactive metrics (e.g. AIx) on LV relaxation. Directly related to this aim is the role of wave reflections in diastolic dysfunction [9, 21, 47, 52]. Studies suggested that earlier arrival of wave reflection during the ejection phase would require the cardiac tissue to generate more tension to overcome the increased afterload $[8,21]$. Increased tension in the LV sarcomeres is known to affect muscle relaxation, which will impair LV filling (i.e. diastolic function). Research on the role of arterial load aspects in the development of LV diastolic dysfunction is clinically relevant, because $\mathrm{LV}$ diastolic dysfunction is a precursor of heart failure, specifically HFpEF [32, 35, 38].

Before we can in silico investigate LV relaxation and the role of arterial load 
aspects, as described above, we first need to address a limitation of our current model. The physiological validity of the whole-heart model [57] that was used in our full circulatory model was limited by the sarcomere contraction model, containing only a phenomenological description of cardiac muscle contraction and relaxation [57]. Recently, the MechChem sarcomere model was proposed, describing mechanical-chemical interactions in cardiac sarcomeres [14]. The MechChem model does take into consideration tension-dependent relaxation, as governed by intracellular calcium concentration [14]. The MechChem model is currently being interfaced to the existing CircAdapt heart modules, which paves the way for studies investigating e.g. the influence of arterial wave reflections on $\mathrm{LV}$ relaxation.

\subsection{Multi-scale modelling perspective and clinical relevance}

In Section 8.3, we have discussed the model developments and applications that have been presented, distinguishing between cardiac, vascular and interaction aspects. Together, the model-based approaches may be combined into a multiscale modelling platform, to study cardiovascular (patho-)physiology from e.g. changes in wall-constituent properties of blood vessels to their impact on the contractile function of cardiac muscle (Fig. 8.3).

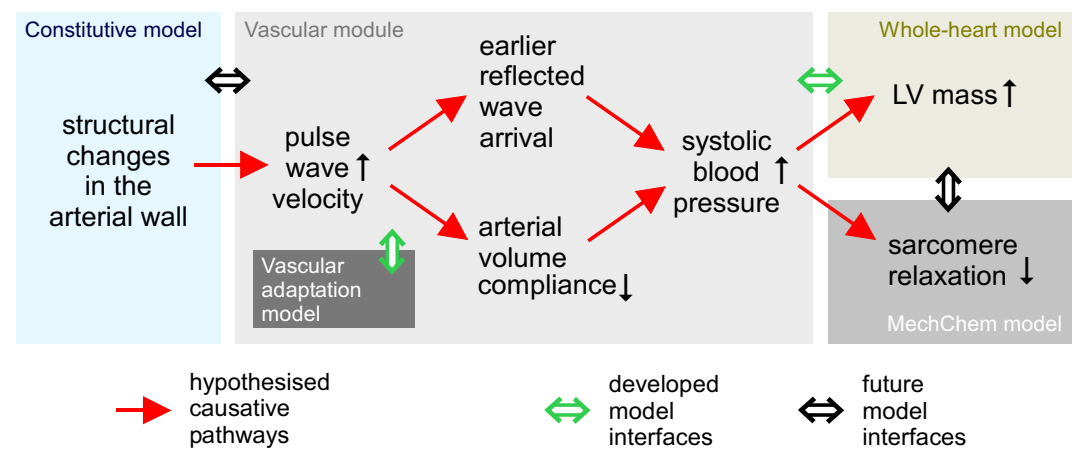

Figure 8.3: Status of development of a multi-scale modelling platform, suited to investigate in silico the indicated hypothesised causative pathways.

The hypothesised causative pathways as indicated in Fig. 8.3 are clinically relevant, because the general population becomes increasingly older. As a result, more patients will likely present with age-related arteriosclerosis (i.e. stiffened arteries) and LV remodelling and/or diastolic dysfunction, affecting medical treatment decisions. Therefore, increased mechanistic insight into cardio-vascular ageing, including well-tested indices that capture functional impairment beyond that expected to come with age, is needed to achieve personalised and sustainable health management. 


\subsection{General conclusions}

In this thesis, we developed, characterised and assessed computational models consisting of networks of applied physical-physiological relations. The models have shown to be helpful in understanding measurable haemodynamic measures as well as enabling estimation of non-measurable properties. As such we have contributed to the integrative interpretation of data which nowadays is at the clinician's disposal. Furthermore, the presented model-components may be combined to form a multi-scale platform to further investigate and characterise pathological heart-vessel interaction in the context of an ageing general population. Ultimately, the developing model platform may serve as a 'digital twin' of the cardiovascular system of a human, for example to assess cardiac effects of vascular drugs. 


\section{References}

[1] H. Aguinis, C. A. Pierce, F. A. Bosco, D. R. Dalton, and C. M. Dalton. Debunking myths and urban legends about meta-analysis. Organizational Research Methods, 14(2):306-331, 2011.

[2] J. Alastruey, K. H. Parker, and S. J. Sherwin. Arterial pulse wave haemodynamics. In 11th International Conference on Pressure Surges, pages 401-442. Virtual PiE Led t/a BHR Group Lisbon, Portugal, 2012.

[3] M. Allon, T. Greene, L. M. Dember, J. A. Vita, A. K. Cheung, N. M. Hamburg, P. B. Imrey, J. S. Kaufman, M. L. Robbin, Y.-T. Shiu, et al. Association between preoperative vascular function and postoperative arteriovenous fistula development. Journal of the American Society of Nephrology, 27(12): 3788-3795, 2016.

[4] T. Arts, J. Lumens, W. Kroon, and T. Delhaas. Control of whole heart geometry by intramyocardial mechano-feedback: a model study. PLOS Computational Biology, 8(2):e1002369, 2012.

[5] A. J. Baksi, T. A. Treibel, J. E. Davies, N. Hadjiloizou, R. A. Foale, K. H. Parker, D. P. Francis, J. Mayet, and A. D. Hughes. A meta-analysis of the mechanism of blood pressure change with aging. Journal of the American College of Cardiology, 54(22):2087-2092, 2009.

[6] E. Boileau, P. Nithiarasu, P. J. Blanco, L. O. Müller, F. E. Fossan, L. R. Hellevik, W. P. Donders, W. Huberts, M. Willemet, and J. Alastruey. A benchmark study of numerical schemes for onedimensional arterial blood flow modelling. International Journal for Numerical Methods in Biomedical Engineering, 31(10), 2015.

[7] A. Caroli, S. Manini, L. Antiga, K. Passera, B. Ene-Iordache, S. Rota, G. Remuzzi, A. Bode, J. Leermakers, F. N. Van De Vosse, et al. Validation of a patient-specific hemodynamic computational model for surgical planning of vascular access in hemodialysis patients. Kidney International, 84 (6):1237-1245, 2013.

[8] J. A. Chirinos, P. Segers, T. C. Gillebert, A. K. Gupta, M. L. De Buyzere, D. De Bacquer, M. St JohnSutton, and E. R. Rietzschel. Arterial properties as determinants of time-varying myocardial stress in humans. Hypertension, 60(1):64-70, 2012.

[9] J. A. Chirinos, P. Bhattacharya, A. Kumar, E. Proto, P. Konda, P. Segers, S. R. Akers, R. R. Townsend, and P. Zamani. Impact of diabetes mellitus on ventricular structure, arterial stiffness, and pulsatile hemodynamics in heart failure with preserved ejection fraction. Journal of the American Heart Association, 8(4):e011457, 2019.

[10] R. Dammers, J. H. M. Tordoir, J. P. Kooman, J. T. J. Rob, J. M. M. Hameleers, P. J. E. H. M. Kitslaar, and A. P. G. Hoeks. The effect of flow changes on the arterial system proximal to an arteriovenous fistula for hemodialysis. Ultrasound in Medicine \& Biology, 31(10):1327-1333, 2005.

[11] H. Den Ruijter, G. Pasterkamp, F. H. Rutten, C. S. P. Lam, C. Chi, K. Tan, A. J. van Zonneveld, M. Spaanderman, and D. P. V. de Kleijn. Heart failure with preserved ejection fraction in women: the Dutch Queen of Hearts program. Netherlands Heart Journal, 23(2):89-93, 2015.

[12] J. Dernellis and M. Panaretou. Aortic stiffness is an independent predictor of progression to hypertension in nonhypertensive subjects. Hypertension, 45(3):426-431, 2005. 
[13] M. H. Drazner. The progression of hypertensive heart disease. Circulation, 123(3):327-334, 2011.

[14] L. J. Dupuis, J. Lumens, T. Arts, and T. Delhaas. High tension in sarcomeres hinders myocardial relaxation: A computational study. PLOS ONE, 13(10):e0204642, 2018.

[15] B. Ene-Iordache, L. Mosconi, L. Antiga, S. Bruno, A. Anghileri, G. Remuzzi, and A. Remuzzi. Radial artery remodeling in response to shear stress increase within arteriovenous fistula for hemodialysis access. Endothelium, 10(2):95-102, 2003.

[16] L. Engelen, C. D. A. Stehouwer, and C. G. Schalkwijk. Current therapeutic interventions in the glycation pathway: evidence from clinical studies. Diabetes, Obesity and Metabolism, 15(8): 677-689, 2013.

[17] M. L. Freidja, K. Tarhouni, B. Toutain, C. Fassot, L. Loufrani, and D. Henrion. The AGE-breaker ALT-711 restores high blood flow-dependent remodeling in mesenteric resistance arteries in a rat model of Type 2 Diabetes. Diabetes, 61(6):1562-1572, 2012.

[18] P. Fridez, M. Zulliger, F. Bobard, G. Montorzi, H. Miyazaki, K. Hayashi, and N. Stergiopulos. Geometrical, functional, and histomorphometric adaptation of rat carotid artery in induced hypertension. Journal of Biomechanics, 36(5):671-680, 2003.

[19] W. H. Gaasch and M. R. Zile. Left ventricular structural remodeling in health and disease: with special emphasis on volume, mass, and geometry. Journal of the American College of Cardiology, 58(17):1733-1740, 2011.

[20] A. Ganau, R. B. Devereux, M. J. Roman, G. De Simone, T. G. Pickering, P. S. Saba, P. Vargiu, I. Simongini, and J. H. Laragh. Patterns of left ventricular hypertrophy and geometric remodeling in essential hypertension. Journal of the American College of Cardiology, 19(7):1550-1558, 1992.

[21] T. C. Gillebert and W. Lew. Influence of systolic pressure profile on rate of left ventricular pressure fall. American Journal of Physiology-Heart and Circulatory Physiology, 261(3):H805-H813, 1991.

[22] W. Grossman, D. Jones, and L. McLaurin. Wall stress and patterns of hypertrophy in the human left ventricle. The Journal of Clinical Investigation, 56(1):56-64, 1975.

[23] A. W. Haider, M. G. Larson, E. J. Benjamin, and D. Levy. Increased left ventricular mass and hypertrophy are associated with increased risk for sudden death. Journal of the American College of Cardiology, 32(5):1454-1459, 1998.

[24] J. Hashimoto, B. E. Westerhof, N. Westerhof, Y. Imai, and M. F. O’Rourke. Different role of wave reflection magnitude and timing on left ventricular mass reduction during antihypertensive treatment. Journal of Hypertension, 26(5):1017-24, 2008.

[25] K. Hayashi, H. Handa, S. Nagasawa, A. Okumura, and K. Moritake. Stiffness and elastic behavior of human intracranial and extracranial arteries. Journal of Biomechanics, 13(2):175-184, 1980.

[26] E. Hermeling, K. D. Reesink, A. P. G. Hoeks, and R. S. Reneman. Potentials and pitfalls of local PWV measurements. American Journal of Hypertension, 23(9):934-934, 2010.

[27] M. H. G. Heusinkveld, S. Quicken, R. J. Holtackers, W. Huberts, K. D. Reesink, T. Delhaas, and B. Spronck. Uncertainty quantification and sensitivity analysis of an arterial wall mechanics model for evaluation of vascular drug therapies. Biomechanics and Modeling in Mechanobiology, 17(1):55-69, 2018.

[28] R. J. Holtackers, B. Spronck, M. H. G. Heusinkveld, G. Crombag, J. Op 't Roodt, T. Delhaas, M. E. Kooi, K. D. Reesink, and E. Hermeling. Head orientation should be considered in ultrasound studies on carotid artery distensibility. Journal of Hypertension, 34(8):1551-1555, 2016. 
[29] A. D. Hughes, C. Park, J. Davies, D. Francis, S. A. M. Thom, J. Mayet, and K. H. Parker. Limitations of augmentation index in the assessment of wave reflection in normotensive healthy individuals. PLOS ONE, 8(3):e59371, 2013.

[30] J. D. Humphrey, D. G. Harrison, C. A. Figueroa, P. Lacolley, and S. Laurent. Central artery stiffness in hypertension and aging: a problem with cause and consequence. Circulation Research, 118 (3):379-381, 2016.

[31] A. Kamiya and T. Togawa. Adaptive regulation of wall shear stress to flow change in the canine carotid artery. American Journal of Physiology-Heart and Circulatory Physiology, 239(1):H14-H21, 1980.

[32] G. C. Kane, B. L. Karon, D. W. Mahoney, M. M. Redfield, V. L. Roger, J. C. Burnett, S. J. Jacobsen, and R. J. Rodeheffer. Progression of left ventricular diastolic dysfunction and risk of heart failure. Journal of the American Medical Association, 306(8):856-863, 2011.

[33] D. A. Kass, E. P. Shapiro, M. Kawaguchi, A. R. Capriotti, A. Scuteri, R. C. deGroof, and E. G. Lakatta. Improved arterial compliance by a novel advanced glycation end-product crosslink breaker. Circulation, 104(13):1464-1470, 2001.

[34] W. Kroon, W. Huberts, M. Bosboom, and F. van de Vosse. A numerical method of reduced complexity for simulating vascular hemodynamics using coupled 0D lumped and 1D wave propagation models. Computational and Mathematical Methods in Medicine, 2012(156094), 2012.

[35] C. S. P. Lam, E. Donal, E. Kraigher-Krainer, and R. S. Vasan. Epidemiology and clinical course of heart failure with preserved ejection fraction. European Journal of Heart Failure, 13(1):18-28, 2011.

[36] G. J. Langewouters, K. H. Wesseling, and W. J. A. Goedhard. The static elastic properties of 45 human thoracic and 20 abdominal aortas in vitro and the parameters of a new model. Journal of Biomechanics, 17(6):425-435, 1984.

[37] B. L. Langille and F. O'Donnell. Reductions in arterial diameter produced by chronic decreases in blood flow are endothelium-dependent. Science, 231(4736):405-407, 1986.

[38] L. Mandinov, F. R. Eberli, C. Seiler, and O. M. Hess. Diastolic heart failure. Cardiovascular Research, 45(4):813-825, 2000.

[39] T. Matsumoto and K. Hayashi. Stress and strain distribution in hypertensive and normotensive rat aorta considering residual strain. Journal of Biomechanical Engineering, 118(1):62-73, 1996.

[40] G. F. Mitchell, H. Parise, E. J. Benjamin, M. G. Larson, M. J. Keyes, J. A. Vita, R. S. Vasan, and D. Levy. Changes in arterial stiffness and wave reflection with advancing age in healthy men and women: the Framingham Heart Study. Hypertension, 43(6):1239-1245, 2004.

[41] J. P. Mynard and J. J. Smolich. One-dimensional haemodynamic modeling and wave dynamics in the entire adult circulation. Annals of Biomedical Engineering, 43(6):1443-1460, 2015.

[42] J. P. Mynard, R. Kowalski, M. M. H. Cheung, and J. J. Smolich. Beyond the aorta: partial transmission of reflected waves from aortic coarctation into supra-aortic branches modulates cerebral hemodynamics and left ventricular load. Biomechanics and modeling in mechanobiology, 16(2): 635-650, 2017.

[43] S. F. Nagueh, O. A. Smiseth, C. P. Appleton, B. F. Byrd, H. Dokainish, T. Edvardsen, F. A. Flachskampf, T. C. Gillebert, A. L. Klein, P. Lancellotti, et al. Recommendations for the evaluation of left ventricular diastolic function by echocardiography: an update from the American Society of Echocardiography and the European Association of Cardiovascular Imaging. European Journal of Echocardiography, 17(12):1321-1360, 2016. 
[44] M. F. O’Rourke and W. W. Nichols. Aortic diameter, aortic stiffness, and wave reflection increase with age and isolated systolic hypertension. Hypertension, 45(4):652-658, 2005.

[45] C. D. Owens, N. Wake, J. Min Kim, D. Hentschel, M. S. Conte, and A. Schanzer. Endothelial function predicts positive arterial-venous fistula remodeling in subjects with stage IV and V chronic kidney disease. The Journal of Vascular Access, 11(4):329-334, 2010.

[46] S. Pagoulatou and N. Stergiopulos. Evolution of aortic pressure during normal ageing: A modelbased study. PLOS ONE, 12(7):e0182173, 2017.

[47] V. R. Peterson, A. J. Woodiwiss, C. D. Libhaber, A. Raymond, P. Sareli, and G. R. Norton. Cardiac diastolic dysfunction is associated with aortic wave reflection, but not stiffness in a predominantly young-to-middle-aged community sample. American Journal of Hypertension, 29(10): 1148-1157, 2016.

[48] M. A. Quail, R. Short, B. Pandya, J. A. Steeden, A. Khushnood, A. M. Taylor, P. Segers, and V. Muthurangu. Abnormal wave reflections and left ventricular hypertrophy late after coarctation of the aorta repair. Hypertension, 69:501-509, 2017.

[49] K. D. Reesink and B. Spronck. Constitutive interpretation of arterial stiffness in clinical studies -a methodological review. American Journal of Physiology-Heart and Circulatory Physiology, 316(3): H693-H709, 2018.

[50] P. Reymond, F. Merenda, F. Perren, D. Rüfenacht, and N. Stergiopulos. Validation of a onedimensional model of the systemic arterial tree. American Journal of Physiology-Heart and Circulatory Physiology, 297(1):H208-H222, 2009.

[51] P. Segers, N. Stergiopulos, J. J. Schreuder, B. E. Westerhof, and N. Westerhof. Left ventricular wall stress normalization in chronic pressure-overloaded heart: a mathematical model study. American Journal of Physiology-Heart and Circulatory Physiology, 279(3):H1120-H1127, 2000.

[52] J. E. Sharman, B. A. Haluska, Z. Y. Fang, J. B. Prins, and T. H. Marwick. Association of arterial wave properties and diastolic dysfunction in patients with type 2 diabetes mellitus. The American Journal of Cardiology, 99(6):844-848, 2007.

[53] G. Sommer and G. A. Holzapfel. 3D constitutive modeling of the biaxial mechanical response of intact and layer-dissected human carotid arteries. Journal of the Mechanical Behavior of Biomedical Materials, 5(1):116-128, 2012.

[54] B. Spronck. Stiff vessels approached in a flexible way: Advancing quantification and interpretation of arterial stiffness. PhD thesis, Maastricht University, 2016.

[55] D. Susic, J. Varagic, and E. D. Frohlich. Cardiovascular and renal effects of a collagen cross-link breaker (ALT 711) in adult and aged spontaneously hypertensive rats. American Journal of Hypertension, 17(4):328-333, 2004.

[56] A. W. van Gorp, D. S. V. I. Schenau, A. P. G. Hoeks, H. A. J. S. Boudier, J. G. R. de Mey, and R. S. Reneman. In spontaneously hypertensive rats alterations in aortic wall properties precede development of hypertension. American Journal of Physiology-Heart and Circulatory Physiology, 278(4):H1241-H1247, 2000.

[57] J. Walmsley, T. Arts, N. Derval, P. Bordachar, H. Cochet, S. Ploux, F. W. Prinzen, T. Delhaas, and J. Lumens. Fast simulation of mechanical heterogeneity in the electrically asynchronous heart using the multipatch module. PLOS Computational Biology, 11(7):e1004284, 2015.

[58] M. Wang, J. Zhang, R. Telljohann, L. Jiang, J. Wu, R. E. Monticone, K. Kapoor, M. Talan, and E. G. Lakatta. Chronic matrix metalloproteinase inhibition retards age-associated arterial proinflammation and increase in blood pressure. Hypertension, 60(2):459-466, 2012. 
[59] T. Weber and A. Protogerou. Left ventricular hypertrophy, arterial stiffness and blood pressure: exploring the Bermuda Triangle. Journal of Hypertension, 37(2):280-281, 2019.

[60] M. Willemet, P. Chowienczyk, and J. Alastruey. A database of virtual healthy subjects to assess the accuracy of foot-to-foot pulse wave velocities for estimation of aortic stiffness. American Journal of Physiology-Heart and Circulatory Physiology, 2015.

[61] M. Willemet, S. Vennin, and J. Alastruey. Computational assessment of hemodynamics-based diagnostic tools using a database of virtual subjects: application to three case studies. Journal of Biomechanics, 49(16):3908-3914, 2016.

[62] B. H. R. Wolffenbuttel, C. M. Boulanger, F. R. Crijns, M. S. Huijberts, P. Poitevin, G. N. M. Swennen, S. Vasan, J. J. Egan, P. Ulrich, A. Cerami, et al. Breakers of advanced glycation end products restore large artery properties in experimental diabetes. Proceedings of the National Academy of Sciences, 95(8):4630-4634, 1998.

[63] H. Wolinsky. Long-term effects of hypertension on the rat aortic wall and their relation to concurrent aging changes: morphological and chemical studies. Circulation Research, 30(3): 301-309, 1972.

[64] N. Zonnebeld, W. Huberts, M. M. van Loon, T. Delhaas, and J. H. M. Tordoir. Preoperative computer simulation for planning of vascular access surgery in hemodialysis patients. The Journal of Vascular Access, 18(1_suppl):S118-S124, 2017. 



\section{Summary}

O

UR cardiovascular system enables transportation of dissolved gases, hormones and other substances to support metabolism, growth, and repair The system consists of an actively contracting heart, which ejects blood into conducting pulmonary and systemic blood vessels. Usually, the heart's left ventricle and the large arteries are coupled in such a way that the volume and rate of the ejected blood match the impedance of the receiving arteries. This coupling is influenced by changes in cardiac and arterial structure and function, which are interrelated through haemodynamic interactions. Therefore, measurable haemodynamic features are of (clinical-epidemiological) interest as markers of cardiovascular health. However, because such markers reflect changes 'inbetween' the interacting heart and arteries, their interpretation may be tricky.

We hypothesised that an integrative description of cardiac and vascular physiology may be better suited to mechanistically explain the interrelationships between cardiac and vascular structure and function. The development and application of a computational modelling platform to assess heart-vessel interaction is the subject of this thesis.

As a first step in the development of our computational modelling platform, we performed a meta-analysis of clinical literature on heart-vessel interaction through treatment-induced changes in arterial properties on the one hand and left ventricular (LV) structure on the other hand (Chapter 2). Through meta-analysis, we found that a decrease in arterial stiffness is associated with a reduction in LV mass. Unfortunately, it was not possible to infer any causal relationships between arterial stiffness, blood pressure and LV structure.

To infer causality in heart-vessel interaction, we turned our attention to computational models describing circulatory physiology (Chapter 3) and extended the existing CircAdapt model, a well-characterised whole-heart model, with a new transmission line (TL) model that also allows for an accurate examination of wave transmission and reflections in blood vessels. The governing equations of the TL model originate from those derived in the 1880s, describing signal transduction along telegraph wires. We first verified the numerical framework of the TL model by benchmarking against a previously validated pulse wave propagation model. The results showed good agreement regarding pressure and flow waveforms (i.e. relative errors $\leq 2.9 \%$ for pressure, and $\leq 5.6 \%$ for flow). Subsequently, the performance of the combined CircAdapt-TL model was tested in a use-case, simulating carotid artery wave intensity profiles during normotensive as well as hypertensive conditions. The results of this use-case indicate that the domain of applicability of CircAdapt is extended for detailed studies on heart-vessel interaction if the TL model is included. 
In Chapter 4, we applied the CircAdapt-TL model to scrutinise the validity of the augmentation index (AIx) as a vascular index of increased pulse wave reflections in a stiffer arterial tree. The AIx quantifies a deemed augmentation (i.e. as signalled by an inflection point) of a pressure waveform relative to its peak-to-peak amplitude. In population studies, the AIx increased with age, which is usually interpreted to result from an increase in pulse wave reflections with the age-related stiffening of arteries. We hypothesised that cardiac properties are likely to influence pressure augmentation as well because they too determine the time-course of the arterial pressure waveform. We simulated the isolated and combined influences of myocardial shortening velocity and arterial stiffness on AIx and indeed found that the AIx may depend as much on cardiac as on vascular properties, which goes against the common interpretation of the index.

In the above, our use of models focussed on evaluating hypotheses. A more advanced application would be to predict patient-specific haemodynamic changes and features, which adds the challenge of model personalisation.

Chapter 5 is the first chapter concerning personalisation of computational models. In our previous simulation studies, we prescribed generic vessel geometries for the modelled arterial and veins. Source data describing these geometries came from a collection of independent literature sources. As such, different parts of the modelled vascular tree are based on data from different subjects, differing in age, sex, BMI and other characteristics. Models containing generic descriptions of the human vasculature often appear insufficient for patient-specific predictions. Unfortunately, complete characterisations of blood vessel geometries and properties are usually not available or even attainable in practice. Chapter 5 presents an adaptation model to complement sparse data on arterial radius and wall thickness, utilising rules for the response to wall stress and shear stress. To test our approach, we acquired vascular MRI and ultrasound data sets of arterial wall thicknesses and radii of central and arm segments in ten healthy subjects. Comparison between adaptation model-predicted and measured geometries demonstrated small differences (bias $\pm 2 \mathrm{SD}$ of difference equal to $0.2 \pm 2.6 \mathrm{~mm}$ for arterial radius, and $-140 \pm 557 \mu \mathrm{m}$ for wall thickness, respectively). We believe that our methodology is suitable to complete sparse data sets in patient-specific applications.

The subsequent two chapters employed model-based approaches for the patientspecific characterisation of LV or arterial properties, respectively. In Chapter 6, we used the CircAdapt-TL model to non-invasively estimate LV end-diastolic pressure $\left(p_{\mathrm{ed}}\right)$ and compliance $\left(C_{\mathrm{ed}}\right)$ from routine measurements of brachial artery blood pressure and echocardiographic recordings. We found reasonable agreement between our non-invasive estimates of $p_{\mathrm{ed}}$ and $C_{\mathrm{ed}}$ and those measured by an invasive catheter.

To improve mechanistic interpretation of clinical arterial stiffness data, inverse modelling approaches may be used to estimate tissue properties from measured vessel geometric and dynamic elastic properties. In Chapter 7 , we utilised a constitutive model of carotid artery mechanics, describing the stress-strain behaviours of arterial collagen and elastin. As a prerequisite, we performed extensive uncertainty quantification and sensitivity analysis to investigate how measurement 
errors propagate into estimation errors. We were able to substantiate that the significant uncertainty with a single set of carotid artery ultrasound and pressure data points can be reduced by increasing the number of repeated acquisitions of cyclic diameter (i.e. distension) and of wall thickness (i.e. intima-media thickness).

This thesis concludes with a general discussion (Chapter 8), in which we provide an overall perspective of how the above model developments and personalisation approaches may be further integrated. We envision the role of our developing computational models to facilitate 1) detailed haemodynamic studies, 2) patient-specific estimation of non-measurable characteristics, and 3) in silico evaluation of disease progression and treatment scenarios. 



\section{Valorisation addendum}

O combat cardiovascular disease and enable patients at risk to age in a healthier way, roughly two strategies can be distinguished. The first strategy

consists of the identification of the cellular mechanisms responsible for cardiovascular disease followed by the development of new drugs acting on these cellular mechanisms to halt or even regress the disease ('the biologist's approach'). The second strategy consists of the earlier detection of cardiac and vascular problems by developing diagnostic technologies that characterise the mechanical performance of the heart and vessels ('the engineer's approach').

This thesis advocates the use of computational models that embody human cardiovascular physiology to achieve better characterisation of heart failure and arterial stiffness mechanisms. Ideally, computational models are so realistic that they can be regarded as 'digital twins' of actual patients. The path towards such a digital twin comprises of both model development steps (Chapters 3 and 5) as well as model corroboration steps (Chapters 4, 6, and 7), in which the utility of the model is put to the test. Although an ultra-realistic digital twin of human cardiovascular physiology is not available yet, we contribute substantially to the development of a digital twin. It is therefore of no surprise that the present thesis readily contains significant valorisation potential: First, valorisation of the knowledge obtained in this research may advance society and the academic field as primary target groups. The second valorisation aspect represents economic exploration by transferring knowledge into new products or services through business development.

Below, I provide a discussion on both valorisation aspects.

\section{Target groups}

Pathological changes to the heart and blood vessels impact local cardiac and vascular tissue properties and influence their function. In patients, this harmful process can progress silently for years but will eventually become apparent. Therefore, assessment of tissue properties can benefit timely diagnosis of pathologies precluding cardiovascular disease. However, clinically applicable measurements alone are often insufficient to assess tissue properties. The combination of mechanistic computational models with a well-selected set of clinical measurements may allow for estimation of tissue properties, using an inverse approach, in which the model is 'tuned' to the measurements. In this thesis, we showed that an inverse approach to routine data has real potential in detecting diastolic dysfunction, a precursor of heart failure (Chapter 6). 
Currently, assessment of left ventricular filling pressure in patients relies on a clinical algorithm that is based on three echocardiographic indices. Such an algorithm can discriminate between (four) grades of diastolic dysfunction (i.e. with increasing severity; no-, grade I-, grade II- and grade III diastolic dysfunction) [1], each of them reflecting a category of filling pressure, ranging from low to elevated [2]. However, in the absence of one echocardiographic index and conflicting results of the remaining two indices, the clinical algorithm contains a grey zone in which filling pressure is classified as indeterminate. The model-based method introduced in Chapter 6, -providing actual estimations of filling pressure and diastolic compliance- could be used alongside or concurrently with the existing clinical algorithm. Such methodology comes at hand when confronted with patients in which the clinical algorithm cannot determine filling pressure.

An inverse method may also be used for characterisation of vascular wall tissue. For this purpose, we did not use the circulatory computational model, but rather a more detailed model describing local arterial wall mechanics that incorporated distinct features of collagen and elastin. The method allows identification of the wall constituent that contributes the most to the increase in stiffness of a patient's arteries. As such, these models may be of use in the design of new vascular drugs (Chapter 7).

Parallel to providing personalised estimates, the developed computational models are useful for hypothesis testing. In Chapter 4, our model study challenges a widespread interpretive model in which augmentation index is deemed and promoted as an index for arterial properties. Already, an increasing number of human studies show that augmentation index might also be influenced by ventricular function and thus cannot serve as a distinct marker of arterial stiffness or wave reflection magnitude. Our study provided yet another important piece of evidence that the augmentation index does not solely reflect arterial stiffness.

While the research version of the circulatory computational model we used is implemented in a scientific programming environment (MATLAB, The Mathworks, Natick, USA), a stripped-down version of the model is also available on-line as a user-friendly 'CircAdapt simulator'. This simulator is actively used for teaching cardiovascular physiology in the (bio-)medical curricula at Maastricht University and many other universities. Currently, the simulator is capable of describing a catalogue of cardiac pathologies, including cardiogenic shock, pulmonary arterial hypertension, and valvular defects. Integration of the vascular module (Chapter 3 ) that models pressure and flow phenomena in the systemic arterial and venous circulations, further extends this catalogue. The present work paves the way for simulation-based education with even more (vascular) types of pathology. Examples of pathologies as well as their recommended clinical assessment methods include, e.g. 1) arterial stiffening; quantifiable using carotid-to-femoral pulse wave velocity, and 2) peripheral arterial disease; assessable using the brachial-ankle index, respectively. 


\section{Utilisation of knowledge for the development of products or businesses}

First, the circulatory computational model is of interest to medical device and (health) technology companies. For example, Samsung and Apple have shown interest in developing wearables (e.g. smartwatches) to be used for continuous measurement of the arterial pulse waveform. Samsung's approach uses a smartwatch to measure the photoplethysmogram (PPG) at the location of the radial artery [3]. Specific features of the wrist-measured PPG waveform are hypothesised to be indicative of vascular conditions such as arteriosclerosis, but also of psychological conditions, including mental stress. Both arteriosclerosis and mental stress are known risk factors of cardiovascular disease [4]. Information from continuous monitoring of arteriosclerosis and mental stress could thus be used to identify patients at risk of cardiovascular disease. Computational models like ours are very well suited to (pre-)test and select sets of PPG features that are sensitive for (changes in) arterial and mental health.

Second, the developed methodologies for determining on the one hand left ventricular diastolic indices and on the other hand, arterial wall constituent properties are already well-suited to be integrated as separate cardiac- or vascular workflows in ultrasound machines. When selecting either of the workflows, the corresponding computational model should be initiated, for example as a thirdparty application, and the user should be guided to perform the measurements that are required to complete or augment the analysis. These computational model approaches to the interpretation of echocardiographic-, and blood pressure data are of interest to companies, such as Microlife Corporation, IMEC, Pie Medical Imaging, and Philips Medical Systems. Partnerships with these companies can be beneficial in two ways. First, third-party integration projects will generate revenue for the Department of Biomedical Engineering, which can be allocated to the hiring of staff or investments in computational resources. Second, partnerships could also include setting up new research and development projects, thereby providing opportunities to further develop our algorithms (e.g. using machine learning methods based on large clinical data sets). 



\section{References}

[1] O. S. Andersen, O. A. Smiseth, H. Dokainish, M. M. Abudiab, R. C. Schutt, A. Kumar, K. Sato, S. Harb, E. Gude, E. W. Remme, et al. Estimating left ventricular filling pressure by echocardiography. Journal of the American College of Cardiology, 69(15):1937-1948, 2017.

[2] S. F. Nagueh, O. A. Smiseth, C. P. Appleton, B. F. Byrd, H. Dokainish, T. Edvardsen, F. A. Flachskampf T. C. Gillebert, A. L. Klein, P. Lancellotti, et al. Recommendations for the evaluation of left ventricular diastolic function by echocardiography: an update from the American Society of Echocardiography and the European Association of Cardiovascular Imaging. European Journal of Echocardiography, 17(12):1321-1360, 2016.

[3] P. Sangyun, K. Younho, Y. Kwon, and N. Seungwoo. Apparatus and method for measuring bioinformation, Apr. 6 2017. US Patent App. 15/284,907.

[4] H. Song, F. Fang, F. K. Arnberg, D. Mataix-Cols, L. F. de la Cruz, C. Almqvist, K. Fall, P. Lichtenstein, G. Thorgeirsson, and U. A. Valdimarsdóttir. Stress related disorders and risk of cardiovascular disease: population based, sibling controlled cohort study. BMJ, 365:11255, 2019. 



\section{Acknowledgments (Dankwoord)}

Bon... U leest hier toch echt in mijn voltooide proefschrift. Het is een behoorlijke reis geweest. Een weg die ik zeker niet alleen heb afgelegd. Ik ben iedereen dankbaar die op de een of andere manier heeft bijgedragen aan het voltooien van dit boekje. Maar nu terzake, want zonder deze tegeltjeswijsheid redden we het ook wel.

Allereerst wil ik mijn promotor en copromotors bedanken.

Beste Tammo, je bent een voortreffelijke leermeester geweest. Jouw aanstekelijk enthousiasme en je directheid hebben ervoor gezorgd dat ik op de momenten waar het nodig was nog een versnelling hoger schakelde. Je oog voor detail (inclusief lessons in vigilance) en je snelle manier van commentaar geven zijn zowel het behalen van deadlines alsmede de kwaliteit van dit proefschrift ten goede gekomen. Tammo, bedankt voor het zijn van mijn promotor.

Beste Koen, je wekte mijn interesse voor het onderzoek rondom hart-vaat interactie en vaatwandmechanica. Ik wil je bedanken voor alle tijd die je in het voltooien van dit PhD-traject hebt gestoken en de kansen die je me hebt gegeven om me verder te kunnen ontwikkelen, bijvoorbeeld in het uitvoeren van onderwijstaken. Met plezier kijk ik nu terug op de momenten waarop je met relativerende, vaak filosofische woorden, een vastgelopen "ik" wist vlot te trekken. Mocht dat laatste met woorden niet lukken, dan lukte dat middels een partijtje squash wel.

Wouter, je kwam halverwege de rit mijn promotieteam versterken. Al ruim daarvoor konden we lange discussies voeren over Womersley profielen en gevoeligheidsanalyse. Jouw expertise op het gebied van patiënt-specifiek modelleren komt zeker terug in dit proefschrift. Wouter, bedankt!

Beste prof. Brunner-La Rocca, prof. Dekker, prof. Duncker, prof. Mischi en dr. Rennenberg, dank jullie wel voor jullie tijd om mijn proefschrift door te nemen en plaats te nemen in de beoordelingscie.

Bart, toen ik als stagiair in het BMT-lab kwam te zitten ben ik bij je in de leer mogen gaan. Wat mooi dat dit boekje twee gezamenlijke publicaties van ons bevat!

Beste Theo, ontzettend bedankt voor je wijze lessen en je scherpe pen. Zonder jouw werk aan het golfvoortplantingsmodel had dit boekje er heel anders uitgezien. Joost, als mede-Kleesj-alumnus kwam ik natuurlijk met de juiste papieren op het BMT-lab te zitten. Het is mooi om te zien hoe jij je CircAdapt-club steeds verder weet uit te bouwen. Bedankt dat ik ook (een klein beetje) deel van je club heb mogen uitmaken.

I would like to thank prof. Alun Hughes of UCL for our collaborations, amongst others, on the non-invasive pressure-volume loop project. The arterial haemodynamics conference in London which you co-organised was one of the most valuable 
experiences I had in my PhD. The opportunity to not only present my work but also attend the lectures of the eminent scientists; profs. Westerhof, Parker, and O'Rourke is something I will never forget.

Beste Bouke, Jos en Rob, toen ik begin 2018 bij jullie aanklopte om te vragen of er (met redelijke urgentie) een vasculaire ultrageluid/MRI-data set vergaard kon worden waren jullie meteen aan boord. Dankzij jullie inzet hebben we in rap tempo de benodigde data bij elkaar verzameld, waardoor hoofdstuk 5 van dit boekje er uiteindelijk van gekomen is. Oja, en Rob, ik wil je ook nog bedanken voor je aandeel in alle ongein die we bij menig (studenten-)feestje hebben uitgehaald. Het gezegde "ik wou dat ik naar huis wou" was hierbij veelvuldig van toepassing.

Ik wil alle BMT-ers die voor zo'n prettige werksfeer zorgden bedanken. Beste Ben, Erik, Letty, Mehrdad, Myrthe, Nick, Pamir en Raoul, jullie zijn samen een superleuk clubje mensen! Natuurlijk wil ik Kujtim en Tamara als collega's van weleer maar bovenal studiemaatjes niet vergeten te bedanken. Beste Claire, dankzij jouw organisatietalent kon ik me haast volledig richten op het onderzoek. Jeroen, het was echt top om met je te kunnen praten over het ultrageluidverleden van de afdeling.

Beste Ciprian, Kenny en Koen vdW, veel succes met jullie carrières. Ik hoop dat jullie met plezier terugkijken op jullie stage, afstudeeropdracht of Erasmus project.

BM2Wielers, squashers en voetballers (Alberto, Aurore, Frank B, Frank vR, Job, Niek en Tijmen): Dank jullie wel voor alle sportieve momenten en gezelligheid.

Een hoogtepunt van de winter was de PhD ski-trip waarin we met goed (internationaal) gezelschap richting de Alpen trokken. I'd like to thank Lauren, Margaux, Martijn, Peter, Radek, and all the other nice people who joined these trips over the years. You guys made it really enjoyable. Whether it was tossing business cards on the ski slopes during the day, or throwing ski helmets across the bar at night, it was always a great laugh.

Een bijzonder dankjewel aan mijn paranimfen. Beste Koen vK, we kennen elkaar al ons hele leven. Wat mooi dat we, ondanks dat we elkaar soms tijden niet spreken, zulke goede vrienden zijn gebleven. Beste Sjeng, naast het zijn van mijn "go-to" collega vind ik het ook fantastisch om buiten het werk met je te praten over muziekinstrumenten, underground optredens en al het moois gemaakt vóór 1990.

Als laatste wil ik mijn familie bedanken. Lieve mama, papa en Lena, jullie waren zo belangrijk de afgelopen jaren. 


\section{About the author}

\section{Curriculum vitae}

$\mathrm{M}$

AARTEN Heusinkveld was born on 28 March 1991 in Sittard, the Netherlands. From 2003 to 2009, he attended atheneum (pre-university education) at Trevianum Scholengroep in Sittard. He started his academic education in September 2009 at Eindhoven University of Technology (TU/e). During his undergraduate studies, Maarten performed a six-month internship at the Department of Biomedical Engineering, Maastricht University (UM). In Maastricht, he discovered that his principal interest lies in the interface between engineering and clinical research. After obtaining his Bachelor's degree in August 2012, he enrolled in the Master Medical Engineering (TU/e in collaboration with UM, 09-2012 to 03-2015). His Master's program included an international internship at the Laboratory of Biorheology and Medical Ultrasonics of the Centre de Recherche du CHUM in Montréal (08-2013 to 11-2013). In Canada, he worked in the lab of prof. dr. Guy Cloutier on vulnerable carotid plaque detection using vascular ultrasound strain analysis. Maarten returned to Maastricht for his Master's graduation project on computational modelling of heart-vessel interaction (supervised by dr. ir. Koen D. Reesink and dr. ir. Bart Spronck). In December 2014, Maarten was awarded a Kootstra Talent Fellowship from Maastricht University Medical Centre. This Fellowship enabled him to continue his graduation project as a $\mathrm{PhD}$ candidate at the Department of Biomedical Engineering (UM, 04-2015 to 04-2019). His $\mathrm{PhD}$ research was performed under the supervision of prof. dr. Tammo Delhaas, dr. ir. Koen D. Reesink and dr. ir. Wouter Huberts, and resulted in the present dissertation. As of May 2019, Maarten works as a product manager of cardiac ultrasound analysis software at Pie Medical Imaging. 


\section{Publications in peer-reviewed journals}

1. M. H. G. Heusinkveld, W. Huberts, J. Lumens, T. Arts, T. Delhaas, and K. D. Reesink. Large vessels as a tree of transmission lines incorporated in the circadapt whole-heart model: A computational tool to examine heart-vessel interaction. PLOS Computational Biology, 15(7):e1007173, 2019.

2. M. H. G. Heusinkveld, T. Delhaas, J. Lumens, W. Huberts, B. Spronck, A. D. Hughes, and K. D. Reesink. Augmentation index is not a proxy for wave reflection magnitude: mechanistic analysis using a computational model. Journal of Applied Physiology, 127(2):491-500, 2019.

3. K. M. van der Waaij*, M. H. G. Heusinkveld*, T. Delhaas, A. A. Kroon, and K. D. Reesink. Do treatment-induced changes in arterial stiffness affect left ventricular structure? A meta-analysis. Journal of Hypertension, 37(2): 253-263, 2019.

4. M. H. G. Heusinkveld, S. Quicken, R. J. Holtackers, W. Huberts, K. D. Reesink, T. Delhaas, and B. Spronck. Uncertainty quantification and sensitivity analysis of an arterial wall mechanics model for evaluation of vascular drug therapies. Biomechanics and Modeling in Mechanobiology, 17(1):55-69, 2018.

\section{In revision}

5. M. H. G. Heusinkveld, R. J. Holtackers, B. P. Adriaans, J. Op 't Roodt, T. Arts, T. Delhaas, K. D. Reesink, and W. Huberts. Complementing sparse vascular imaging data by physiological adaptation rules. Journal of Applied Physiology.

\section{In preparation}

6. M. H. G. Heusinkveld, T. Delhaas, K. March, J. E. Davies, S. Sen, J. Mayet, R. A. Bouwman, J. Lumens, A. D. Hughes, and K. D. Reesink. Non-invasive modelbased estimation of left ventricular end-diastolic pressure and compliance.

\section{Not included in this thesis}

7. M. Bloksgaard, T. M. Leurgans, B. Spronck, M. H. G. Heusinkveld, B. Thorsted, K. Rosenstand, I. Nissen, U. M. Hansen, J. R. Brewer, L. A. Bagatolli, et al. Imaging and modeling of acute pressure-induced changes of collagen and elastin microarchitectures in pig and human resistance arteries. American Journal of Physiology-Heart and Circulatory Physiology, 313(1):H164-H178, 2017.

8. M. H. Roy-Cardinal, M. H. G. Heusinkveld, Z. Qin, R. G. P. Lopata, C. Naim, G. Soulez, and G. Cloutier. Carotid artery plaque vulnerability assessment

\footnotetext{
* Equal contributions
} 
using noninvasive ultrasound elastography: Validation with MRI. American Journal of Roentgenology, 209(1):142-151, 2017.

9. R. J. Holtackers, B. Spronck, M. H. G. Heusinkveld, G. Crombag, J. O. 't Roodt, T. Delhaas, M. E. Kooi, K. D. Reesink, and E. Hermeling. Head orientation should be considered in ultrasound studies on carotid artery distensibility. Journal of Hypertension, 34(8):1551-1555, 2016.

10. A. den Hamer, M. H. G. Heusinkveld, W. Traa, P. Oomen, F. Oliva, A. Del Buono, and N. Maffulli. Current techniques for management of transverse displaced olecranon fractures. Muscles, Ligaments and Tendons Journal, 5(2):129-140, 2015.

11. B. Spronck, M. H. G. Heusinkveld, F. H. Vanmolkot, J. Op't Roodt, E. Hermeling, T. Delhaas, A. A. Kroon, and K. D. Reesink. Pressure-dependence of arterial stiffness: potential clinical implications. Journal of Hypertension, 33 (2):330-338, 2015.

12. B. Spronck, M. H. G. Heusinkveld, W. P. Donders, A. G. W. de Lepper, J. Op't Roodt, A. A. Kroon, T. Delhaas, and K. D. Reesink. A constitutive modeling interpretation of the relationship among carotid artery stiffness, blood pressure, and age in hypertensive subjects. American Journal of Physiology-Heart and Circulatory Physiology, 308(6):H568-H582, 2014.

13. W. A. Traa, P. J. A. Oomen, A. den Hamer, M. H. G. Heusinkveld, and N. Maffulli. Biomechanical studies on transverse olecranon and patellar fractures: a systematic review with the development of a new scoring method. British Medical Bulletin, 108(1):131-157, 2013.

14. M. H. G. Heusinkveld, A. den Hamer, W. A. Traa, P. J. A. Oomen, and N. Maffulli. Treatment of transverse patellar fractures: a comparison between metallic and non-metallic implants. British Medical Bulletin, 107(1):69-85, 2013.

\section{Proceedings in peer-reviewed journals}

15. M. H. G. Heusinkveld, M. H. R. Cardinal, Y. Ju, Z. Qin, R. G. P. Lopata, G. Soulez, and G. Cloutier. Carotid plaque assessment using non-invasive shear strain elastography. In 2014 IEEE International Ultrasonics Symposium, pages 117-120. IEEE, 2014. 
